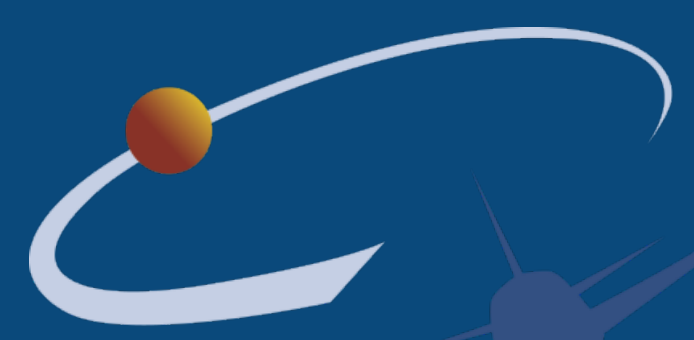

\title{
Direct and Adjoint Methods for Highly Detached Flows
}

\author{
Doctoral Thesis \\ By \\ Alejandro Martínez-Cava Aguilar \\ Aeronautical Engineer
}

July 2019 

Departamento de Matemática Aplicada a la Ingeniería Aeroespacial Escuela Técnica Superior de Ingeniería Aeronáutica y del Espacio

\title{
Direct and Adjoint Methods for Highly Detached Flows
}

\author{
by
}

Alejandro Martínez-Cava Aguilar

Aeronautical Engineer

Advisors:

Eusebio Valero Sánchez

Ph.D. in Aeronautical Engineering

Javier de Vicente Buendía

Ph.D. in Aeronautical Engineering

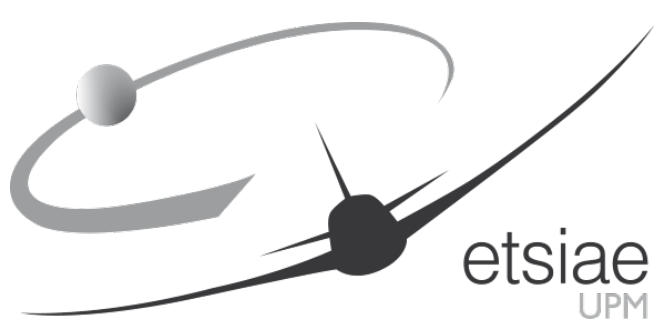

Madrid, July 2019 
This thesis has received funding from the European Union's Horizon 2020 Research and Innovation Program under the Marie Skłodowska-Curie grant agreement No 675008.

Esta tesis ha recibido financiación del programa Horizon 2020 de investigación e innovavión de la Unión Europea bajo el acuerdo de beca número 675008 del programa Marie Skłodowska-Curie. 


\section{POLITÉCNICA}

El tribunal nombrado por el Sr. Rector Magnífico de la Universidad Politécnica de Madrid, el día de de $20 \ldots . .$.

Presidente:

Vocal:

Vocal:

Vocal:

Secretario:

Suplente:

Suplente:

Realizado el acto de defensa y lectura de la Tesis el día de de $20 \ldots$ en la E.T.S.I./Facultad......

Calificación.

EL PRESIDENTE

LOS VOCALES

EL SECRETARIO 



\section{Direct and Adjoint Methods for Highly Detached Flows}

\section{Abstract}

The scope of this work is to approach the study of highly detached flows using hydrodynamic stability theory. In either simple laminar wake flows or complex scenarios, where a turbulent wake is immersed in a system of expansion and compression waves systems, different flow phenomena may arise and generate detrimental aerodynamic effects on the performance of a system. The results of this research aim to extend the physical understanding of detached flows flow instabilities, and to propose possible flow control techniques.

The asymptotic behavior of a flow system is defined by the stability characteristics of the non-linear operator of the Navier-Stokes equations. A combination of CFD calculations to obtain a steady or a periodic state and the use of Modal Stability Theory, permits the linearization of the system and the calculation of the associated large eigenvalue problem. Bifurcation scenarios and the onset of global instabilities are addressed using a Finite Volumes discrete framework for the calculation of the Jacobian matrix of the Navier-Stokes equations. The leading eigenvalues of the matrix and their associated direct and left eigenvectors, the direct and adjoint modes, are recovered and used to address and describe the physical characteristics of the flow system.

The aforementioned procedure for the analysis of the stability has been applied to several complex flows. Among others, the analysis of turbine blades operating at high speed flows. At these conditions the flow develops a complex shock wave system at the trailing edge that can interact with downstream turbine stages. Moreover, colder flow, bled from the high-pressure compressor, is often purged at the trailing edge base region to cool the thin blade edges, affecting the flow behavior and modulating the intensity and angle of the shock waves system. Base pressure control is often employed on drag reduction design, but the interaction of the jet with the base region flow topology can generate undesired or uncontrolled flow configurations. The ejected flow can drive pressure bifurcations at the trailing edge, that without a correct optimization can affect the aerodynamic performance of the system. In this work, a combination of URANS simulations, global stability analysis and adjoint methodologies is employed to fully understand the physical mechanisms related with the use of injected base bleed. Moreover, the results of the sensitivity analysis are used to identify the regions more receptive to passive and active flow control methodologies.

On the study of the sensitivity of the eigenvalues to perturbations, the target of the analysis is normally the least stable global mode, whose amplification rate or associated frequency we aim to reduce or increase. The information contained in the adjoint global modes may be exploited to compute the sensitivity of the eigenvalue to normal displacements of the surface mesh nodes. If this information is used to 
predict surface deformations, it is possible to develop an optimization process with excellent performance on the stabilization/excitation and frequency control of the global mode.

Keywords: Hydrodynamic stability, Bifurcation, Sensitivity analysis, Adjoint methods, Flow control, Compressible Navier-Stokes, Aerospace, Turbine flows, Turbomachinery 


\section{Direct and Adjoint Methods for Highly Detached Flows}

\section{Resumen}

El objetivo de este trabajo es el estudio de flujos altamente desprendidos desde la teoría de estabilidad hidrodinámica. Tanto en estelas laminares como en escenarios más complejos, donde una estela turbulenta está inmersa en un sistema de ondas de compresión y expansión, diferentes fenómenos fluidodinámicos pueden aparecer y generar efectos aerodinámicos adversos en el rendimiento del sistema. Los resultados de este trabajo buscan extender el conocimiento relativo a las inestabilidades presentes en flujos desprendidos y proponer posibles técnicas de control de flujo para paliar sus efectos.

El comportamiento asintótico de un sistema fluido queda definido por las propiedades de estabilidad del operador no lineal de las ecuaciones de Navier-Stokes. Para estudiar dichas propiedades se sigue un esquema que consta de dos partes: En una primera etapa, una combinación de cálculos CFD permiten obtener un estado estacionario o periódico del fluido para a continuación mediante el uso de Teoría de Estabilidad modal, se linealiza el sistema de ecuaciones obteniendo el problema asociado de autovalores. Este análisis permite la identificación de escenarios de bifurcación y la aparición de inestabilidades globales. Los autovalores dominantes de la matriz y sus autovectores asociados por la izquierda o por la derecha -los modos directos y adjuntos, respectivamente- nos permiten identificar e incluso actuar sobre las características físicas del sistema fluido.

Como ya se ha mencionado, este análisis ha permitido estudiar diferentes flujos complejos de naturaleza que hasta ahora estaban vetados a la aplicación más clásica de los análisis de estabilidad lineal. Entre otros el estudio de álabes de turbinas en régimen de operación a altas velocidades. Éstos desarrollan un sistema complejo de ondas de choque en el borde de salida que puede interactuar con otras etapas de la turbina situadas aguas abajo. Al mismo tiempo, un flujo de aire frío extraído del compresor de alta presión es eyectado en la región base del borde de salida para refrigerar los delgados álabes, alterando el fluido aguas abajo y modulando la intensidad y el ángulo del sistema de ondas de choque. El control de presión de base es habitualmente empleado en diseño en la reducción de la resistencia aerodinámica, pero la interacción del chorro de salida con la topología fluida en la región base puede generar configuraciones fluidas no deseadas o controladas. El flujo eyectado puede provocar bifurcaciones de presión en el borde de salida, que sin la correcta optimización pueden afectar el rendimiento aerodinámico del sistema. En este trabajo se ha empleado una combinación de simulaciones no estacionarias (URANS), análisis de estabilidad global y el uso del problema adjunto para comprender los mecanismos físicos relacionados con la inyección de flujo en la región base. Además, los resultados correspondientes del análisis de sensibilidad permiten identificar aquellas regiones más receptivas a técnicas de control de flujo pasivas o activas. 
En el estudio de la sensibilidad de los autovalores a perturbaciones el objetivo del análisis es normalmente el modo global menos estable, cuyo factor de amplificación o frecuencia asociada deseamos incrementar o reducir. La información contenida en los modos globales adjuntos puede ser utilizada para calcular la sensibilidad del autovalor a desplazamientos de los nodos de la malla en dirección normal a la superficie del cuerpo. Si esta información es usada para predecir deformaciones superficiales, es posible desarrollar un proceso de optimización para la estabilización/excitación de un modo global o el control de su frecuencia asociada.

Keywords: Estabilidad hidrodinámica, Bifurcación, Análisis de sensibilidad, Métodos adjuntos, Control de Flujo, Navier-Stokes compresible, Aeroespacial, Flujos de turbinas, Turbomaquinaria 


\section{Acknowledgements}

First, to my supervisors, Eusebio and Javier, for giving me the opportunity of jumping into this project and being always there to answer my doubts. With all the hours dedicated to this thesis, in part it is also yours. I hope to keep learning from the dedication and perspective that you apply to your personal and working life.

For opening her doors for me that Scottish summer and showing me that research life and a getting a $\mathrm{PhD}$ meant far more than I thought, Natacha, thank you. And, of course, to my parents, for bringing me to this world and teaching me the values that have helped me to get where I am.

To my inseparable through thick and thin friends, with which I have shared granite and mountains, dust and chalk, and wine and cheese at the summits. To my lifetime friends, that distance and time have not changed a thing and every day feels like the first one. Thanks for being always there with a smile and a good plan.

To the SSeMID network, colleagues in this international project thanks to which we had the chance to meet and enjoy great experiences through trips and secondments.

Great part of this work would not have been possible without the hospitality of PETAL group, from Purdue University, by the hands of Guillermo Paniagua. Thank you for hosting and treating me as an extra team member during my time in West Lafayette. Likewise, I would like to acknowledge Dr. Denis Sipp and Olivier Marquet for their time and dedication during my secondment at ONERA.

To the whole Applied Mathematics department, from Tere to Mariola stopping in every office, thank you. All those coffees, after-lunch and snack times, and those supportive talks in the terrace, filled me again with motivation when there was a lack of it.

And, without a doubt, thank you, María. For sharing this time with me, with your day-by-day unconditional support. Without you, this would not have been possible. 


\section{Agradecimientos}

En primer lugar, a mis directores de tesis, Eusebio y Javier, por darme la oportunidad de embarcarme en este proyecto y por estar siempre disponibles para solucionar mis dudas. Tantas horas dedicadas a esta tesis también la hace vuestra en cierta manera. Espero poder continuar aprendiendo de vosotros la dedicación y la perspectiva que ponéis tanto a la vida laboral como la personal.

Por abrirme sus puertas aquel verano escocés y mostrarme que la investigación y el doctorado era mucho más de lo que creía, Natacha, mil gracias. Y, por supuesto, a mis padres, por traerme al mundo y por enseñarme grandes valores que me han llevado hasta donde estoy ahora.

A mis inseparables compañeros de fatigas, de granito y montaña, de polvo y magnesio, de vinos y quesos en las cimas. A mis amigos de toda la vida, que la distancia y el tiempo no ha cambiado nada, y cada día es como si fuese el primero. Gracias por estar ahí siempre con una sonrisa y un buen plan.

A la red SSeMID, compañeros de este proyecto internacional gracias al cual hemos podido llegar a conocernos mucho mejor y pasar buenas experiencias a través de viajes y estancias.

Gran parte de este trabajo no hubiese sido posible sin la hospitalidad del groupo PETAL, de Purdue University, de la mano de Guillermo Paniagua. Gracias por acogerme como un miembro más del equipo, y dejarme aprender tanto en el tiempo que pasé en West Lafayette. Asimismo, gracias a los doctores Denis Sipp y Olivier Marquet por su dedicación y ayuda prestada durante mi estancia en ONERA.

A todo el departamento de Matemática Aplicada, desde Tere a Mariola pasando por cada uno de los despachos. Sus cafés, sobremesas y aperitivos, y esas charlas en la terraza que le devuelven a uno la motivación cuando falta.

Y por supuesto, gracias a ti, María. Por acompañarme y apoyarme incondicionalmente día a día todo el camino, sin ti esto no hubiese sido posible. Prometo devolverte todas esas noches, fines de semana y mil ratos que se quedaron en el tintero. 


\section{Contents}

1 Introduction $\quad 3$

1.1 Motivation . . . . . . . . . . . . . . . . . 3

1.2 Historical review and state of the art . . . . . . . . . . 5

1.3 Linear stability analysis . . . . . . . . . . . . . . . . 7

1.4 Thesis objectives and organization . . . . . . . . . . . . . 8

1.5 Novelty of the work and main contributions . . . . . . . . . . . 11

I Theoretical Description \& Numerical Implementation 13

2 Mathematical Development $\quad 15$

2.1 Global Stability Analysis . . . . . . . . . . . . . . . . . 15

2.2 Eigenvalue Sensitivity Analysis . . . . . . . . . . . . . . 21

3 Numerical Implementation $\quad 27$

3.1 Flow Solver - DLR-TAU Code . . . . . . . . . . . . . . . 27

3.2 Stability Analysis - Large Eigenvalue Problem Solver . . . . . . . . . 33

II Validation $\quad 39$

4 Numerical Validation 41

4.1 Laminar Flows . . . . . . . . . . . . . . . . . . . . . . . . 42

4.2 Turbulent Flows . . . . . . . . . . . . . . . . . . . . . . . . . . . 49

III Analysis of High Speed Flows 55

5 Global Stability of a Base Bleed Turbine Blade Trailing Edge $\quad 57$

5.1 Instability detection and characterisation . . . . . . . . . . . 58

5.2 Further Investigation . . . . . . . . . . . . . . . . . . . . . 75 
IV Eigenvalue Sensitivity to Geometrical Changes $\quad 91$

6 Stability Analysis Based Geometry Optimization 93

6.1 Introduction . . . . . . . . . . . . . . . . . . . . 94

6.2 Sensitivity of the eigenvalue to geometry changes . . . . . . . . . 96

6.3 Implementation and validation . . . . . . . . . . . . . . . . . . . 99

6.4 Numerical experiment. The cylinder wake instability . . . . . . . . . 102

6.5 Optimization process . . . . . . . . . . . . . . . . . . 104

6.6 Control of the growth rate of the instability . . . . . . . . . . 106

6.7 Control of the vortex shedding associated frequency . . . . . . . . . 107

6.8 Discussion on the influence of the deformation control parameter, $\varepsilon$. 108

6.9 Analysis and discussion . . . . . . . . . . . . . . . . . . . . . . . . 108

6.10 Conclusions . . . . . . . . . . . . . . . . . . 110

V Conclusions and Future Directions 113

7 Final Discussion and Work Perspectives 115

$\begin{array}{ll}\text { Bibliography } & 119\end{array}$ 


\section{List of Figures}

1.1 Sketch of common flow instabilities. Rayleigh-Bénard convection (a), Kelvin-Helmholtz shear instability (b) and contact line fingering instability (c) . . . . . . . . . . . . . . . . . 4 4

1.2 Da Vinci drawings on his studies of flow turbulence (left, centre). Figures from Reynolds on his analysis of turbulence transition (right). 4

1.3 Types of asymptotic instability . . . . . . . . . . . . 7

3.1 Example of numerical fluxes balance in each direction for a threedimensional hexahedral mesh element. . . . . . . . . . . . . . . . 29

3.2 Compact second-order stencil. Grey cells correspond to the immediate neighbours, and light grey cells are the immediate next-neighbours. Images modified from [35]. . . . . . . . . . . . . . . . . 32

4.1 Streamwise velocity contours of the symmetric base flow solution, at $R e=45$. The streamline of the recirculation area is depicted with a black line. Only part of the computational domain is shown in the image. . . . . . . . . . . . . . . . . . . . 42

4.2 Leading global mode at $R e=45$. Real part of the streamwise velocity (upper image) and vertical velocity (lower image) perturbations. . . . 43

4.3 Leading adjoint global mode at $R e=45$. Real part of the streamwise velocity (upper image) and vertical velocity (lower image) perturbations. . . . . . . . . . . . . . . . . . . 44

4.4 Structural sensitivity of the cylinder wake first instability. Left: Results of the present study. Right: Results from Giannetti and Luchini[46], reprinted under the permission of Cambridge University Press. . . . . 44

4.5 Sensitivity to the application of a steady forcing of the least stable eigenvalue at $R e=45$. Results of present study of the growth rate sensitivity $\boldsymbol{\nabla}_{\overline{\mathbf{q}}} \sigma_{r}$ (upper right) and for the associated frequency $\nabla_{\overline{\mathbf{q}}} \sigma_{i}$ (lower right), and reprinted from Marquet et al.[77](upper and lower left) with the permission of Cambridge University Press. . . . . . . . 45

4.6 Channel sudden expansion geometry. . . . . . . . . . . . . . 46 
4.7 Streamwise velocity contours for the symmetric (left) and non-symmetric (right) flow configurations, at $R e=90 \ldots$. . . . . . . . . . . . 47

4.8 Direct (left) and adjoint(right) global modes of the sudden expansion global instability. . . . . . . . . . . . . . . . . . . . 48

4.9 Sensitivity of the eigenvalue with respect to a structural perturbation. Left: current analysis. Right: results from Ref.[37], Reprinted from Fani et al.[37], with the permission of AIP Publishing. . . . . . . . . . 48

4.10 Sensitivity of the eigenvalue with respect to the application of a steady forcing. Left: current analysis. Right: results from Ref.[37], Reprinted from Fani et al.[37], with the permission of AIP Publishing. 48

4.11 Domain distribution and close-up of the structured mesh used on the calculation of the turbulent flow around the D-shaped cylinder. . . . 50

4.12 Unsteady RANS results for $R e=13000$. Streamwise velocity contours are collected for an aleatory time snapshot (left) and for the time-averaged solution (right) . . . . . . . . . . . . 50

4.13 Flow oscillator main frequency obtained from the URANS simulation (left) and the Linear Stability Analysis (right). . . . . . . . . . . . 51

4.14 Global direct (left) and adjoint (right) modes. Figures show the spatial distribution of the real part of the stream-wise velocity components. Reference figures extracted from Mettot[88]. . . . . . . . . . 51

4.15 Sensitivity analysis of the leading global mode. . . . . . . . . . . . 53

4.16 Streamwise component of the imaginary part of the eigenvalue sensitivity to a steady force. Comparison of the present results (left) with the obtained by Mettot et al.[90], reprinted with the permission of AIP Publishing. . . . . . . . . . . . . . . . . 53

4.17 Sensitivity of the leading global mode to passive flow control. Comparison of the present results (left) with the numerical solution obtained by Meliga et al.[86] (upper half) and the experimental data of Parezanovic and Cadot[101] (lower half), reprinted with the permission of AIP Publishing. . . . . . . . . . . . . . . . .

5.1 Schematic representation of supersonic flow on a blunt trailing edge. Image modified from Saracoglu et al.[122] . . . . . . . . . . . . . 58

5.2 Base pressure correlation at the trailing edge as a function of the density ratio of the bleeding rate. Image reprinted from Saracoglu et

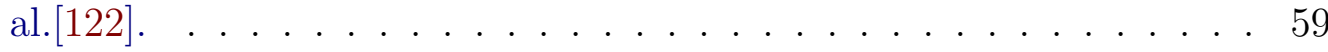

5.3 Geometry definition (solid) and grid topology (dashed-lines) used on the mesh generation. Only half domain is shown for simplification. . . 62

5.4 Computational mesh. Only a quarter of total nodes are represented for clarity. . . . . . . . . . . . . . . . . 63

5.5 Domain reduction technique convergence analysis. . . . . . . . . . . 65

5.6 Density and Pressure values measured at the base region. . . . . . . . 66 
5.7 Base flow computations for different test cases and no bleeding configuration. Pressure contours. . . . . . . . . . . . . . 66

5.8 Base flow computations for different test cases and no bleeding configuration. Streamwise velocity contours. . . . . . . . . . . 67

5.9 Dead air regions for different test cases and no bleeding configuration. Only negative velocities values are plotted. . . . . . . . . . 68

5.10 Shadowgraph contours for non-symmetric (a) and symmetric (b) flow configurations. . . . . . . . . . . . . . . . . . . . . . . . . 69

5.11 Evolution of the eigenvalue spectrum for a stable (17.5\%) and an unstable $(18.5 \%)$ configuration. . . . . . . . . . . . . . 69

5.12 Relation between the global mode and the flow topology. . . . . . . . 70

5.13 Evolution of the streamwise component of the eigenmode with the blowing rate. . . . . . . . . . . . . . . . . 71

5.14 Sensitivity gradients of the leading eigenvalue. Structural sensitivity (left) and sensitivity to steady forcing (right), $\nabla_{\mathbf{q}_{\mathbf{f}}} \sigma$. here shown as the module of the momentum components. . . . . . . . . . . . 71

5.15 Breakdown by primitive variables of the sensitivity gradients of the leading eigenvalue to changes in the base flow. . . . . . . . . . . 72

5.16 Breakdown by primitive variables of the sensitivity gradients of the leading eigenvalue to the application of a steady forcing. . . . . . . . 73

5.17 Comparison of trailing edge shapes used on the analysis. Left image shows the "straight" (super ellipse) shape, with the "rounded" (ellipse) shape on the right image. . . . . . . . . . . . . . . 77

5.18 Mesh topology used for the different sonic regimes: Subsonic (left), transonic (centre), supersonic (right) . . . . . . . . . . 78

5.19 FFT analysis (left) of the lift coefficient sampling from an unsteady subsonic simulation (right). . . . . . . . . . . . . . 78

5.20 Temporal snapshot of static pressure contours for subsonic (left), transonic (center), and supersonic (right) regimes. Base bleed not active. . . . . . . . . . . . . . . . . 80

5.21 Base pressure evolution with the base bleed intensity in subsonic regime. Results for straight (upper) and rounded (lower) trailing edge geometries. . . . . . . . . . . . . . . . . . 81

5.22 Temporal snapthot of streamwise velocity contours for base bleed non-symmetrical flow configurations (phase II), caused by the global instability of the sudden expansion. Upper row shows straight trailing edge flow solutions (subsonic to supersonic, from left to right), and bottom row shows the rounded trailing edge flow solutions. . . . . . . 81

5.23 Global mode related with the geometrical sudden expansion, subsonic regime. Streamwise $(u)$ and vertical $(w)$ velocity perturbations are shown for both trailing edge configurations. 
5.24 Base pressure evolution with the base bleed intensity in transonic regime. Results for straight (upper) and rounded (lower) trailing edge geometries. . . . . . . . . . . . . . . . . 83

5.25 Base pressure evolution with the base bleed intensity in supersonic regime. Results for straight (upper) and rounded (lower) trailing edge geometries.

5.26 Global mode related with the geometrical sudden expansion, supersonic regime. Streamwise $(u)$ and vertical $(w)$ velocity perturbations are shown for both trailing edge configurations. . . . . . . . . . . . 86

5.27 Structural sensitivity of the global mode responsible of the non-symmetrical flow configurations. Left two figures (upper and lower) correspond to subsonic conditions, with the right two figures obtained for supersonic flow.

5.28 Sensitivity to the application of a steady force. Perturbations in the direction of the streamlines would have a destabilizing effect on the eigenvalue. Left two figures (upper and lower) correspond to subsonic conditions, with the right two figures obtained for supersonic flow. .

5.29 Localization of regions where the location of a small control cylinder will generate a major drift on the eigenvalue. Left two figures (upper and lower) correspond to subsonic conditions, with the right two figures obtained for supersonic flow. . . . . . . . . . . . . . . .

6.1 Compact second-order stencil used on the calculation of the Jacobian and the residuals. The dark grey cell indicates the wall cell that is deformed, grey cells correspond to the immediate neighbours, and light grey cells are the immediate next-neighbours. . . . . . . . . . . . 98

6.2 Mathematical procedure for the calculation of the sensitivity to mesh nodes displacements. . . . . . . . . . . . . . . . . . . . . . 100

6.3 Comparison between the analytic and numerical sensitivity of the eigenvalue to changes in $\epsilon_{2}$ and the parameter $p . . . . . . . . .102$

6.4 (a): Unstructured H-Grid used for the simulations, overlapped with vorticity magnitude contours $(R e=60)$. Only half of the mesh nodes are shown. (b): Eigenvalue distribution for the laminar flow over a cylinder, $R e=60 \ldots \ldots \ldots$. . . . . . . . . . . . 103

6.5 Sensitivity of the eigenvalue to outward normal displacements of the surface nodes, as a function of the circular cylinder azimuth (left). Node distribution of the sensitivity of the amplification rate (center) and the sensitivity of the pulsation (right) of the eigenvalue. . . . . . 105

6.6 Optimization loop. . . . . . . . . . . . . . . . . . 106

6.7 Eigenvalue real and imaginary parts for the reference and optimized geometries (left and center images), and final shapes of the body after the optimization (right). $-*$ : reference; $\rightarrow$ : minimum amplification rate; -•: maximum amplification rate. . . . . . . . . . . . . 107 
6.8 Eigenvalue real and imaginary parts for the reference and optimized geometries (left and center images), and final shapes of the body after the optimization (right). $-*$ : reference; $-\triangleright$ : minimum pulsation; -•: maximum pulsation. . . . . . . . . . . . . . 108

6.9 Convergence study for difference deformation ratios. (a) Behavior of the real part of the eigenvalue with respect to the number of iterations depending on the deformation ratio, and (b) final shape after the optimization process. 3stdev denotes three standard deviations. . . . 109

6.10 Non-steady solutions for a $R e=60$ laminar flow configuration. Lift coefficient monitors for (a) reference cylinder, (b) optimized shape for minimum amplification rate and (c) optimized shape for minimum frequency. . . . . . . . . . . . . . . . . . 109

6.11 Streamwise velocity contours for a symmetrical steady flow. Final optimized surface shapes for a minimum(center)/maximum(right) eigenvalue amplification rate, at $R e=60$. Contours for the reference geometry are plotted on the left picture. . . . . . . . . . . . . . . . 110

6.12 Streamwise velocity contours for a symmetrical steady flow. Final optimized surface shapes for a minimum(center)/maximum(right) eigenvalue pulsation, at $R e=60$. Contours for the reference geometry are plotted on the left picture. . . . . . . . . . . . . . . . . 110 



\section{List of Tables}

4.1 Eigenvalue associated frequency literature revision. . . . . . . . . . . 43

4.2 Recirculation bubble length literature revision. Units non-dimensionalised with the inlet height. . . . . . . . . . . . . . . . . . 47 47

4.3 Characteristic frequency of the oscillating wake of the D-shaped cylinder, $R e=13000$. Data from URANS or experimental analysis are compared against Linear Stability Analysis results. . . . . . . . . . 52

5.1 Fluid domain dimensions . . . . . . . . . . . . . . . . . . . . 61

5.2 Mesh Convergence Analysis performed for a blowing rate of $18 \%$ for a half-domain configuration. $P_{b} / P_{b}^{*}$ represented the relation between the pressure with and without purge flow applied, and $\rho_{\text {purge }} / \rho_{f}$ was the relation between the density values at the cavity plenum and at the base region. . . . . . . . . . . . . . . . 63

6.1 Summary of amplification rate and pulsation values for $R e=60 \ldots .110$ 



\section{Preface}

Like many doctorate studies, the main direction of this research "drifted" a couple of times in these three years. While the main topic, direct and adjoint methods on hydrodynamic stability, was established under the European project to which this $\mathrm{PhD}$ belongs, the final outcome was alive and open to modifications until the half term of the second year of the PhD itself. Being part of the International Training Network SSeMID (Stability and Sensitivity Methods for Industrial Design) also provided me with continuous courses and resources that contributed on the progress of the $\mathrm{PhD}$ and gave me the tools (with both technical and transversal skills) to properly address those "drifts".

The initial research line was the extension of two-dimensional stability analysis on a discrete framework, to a real BiGlobal analysis, where the spanwise periodicity of the perturbations is taken into account. A combination of lack of success on this point, and the apparition of a collaboration research with Purdue University (West Lafayette, Indiana, USA), led to pause this research topic for some time. Interestingly, only a couple of months after this research pause, Schmid et al.[127] published an article on the stability analysis of periodic systems, where this topic was further extended and developed. I could say we were in the right direction, but unfortunately without reaching port in time. The started collaboration with Purdue University did, however, lead this $\mathrm{PhD}$ research towards more specific aerospace related scenarios. Indeed, two indexed publications were published in 2018 from that initial point of contact $[81,82]$. At the end of 2018, I completed a three-months PhD secondment at Purdue University, within the Purdue Experimental Turbine Aerothermal Laboratory group (PETAL). In those months, the initial collaboration started in 2017 became a larger and long term project involving numerical and experimental analysis. In the time I spent there, a numerical preliminary study was completed and finally submitted for publication and presentation at the AIAA SciTech Forum 2019[79]. The continuation of this project has so far given another publication[80], stepping into the flow control field using stability analysis as a starting point.

A publication of Wang et al.[153] (another SSeMID fellow and co-worker at UPM) in 2018, on the capabilities of stability analysis on geometry optimization problems using genetic algorithm techniques, suggested the need of further investi- 
gation on the sensitivity of the Navier-Stokes stability problem to geometry modifications. This research line was opened and, at the beginning of 2019, I was able to submit a publication on this topic for journal publication. Right after the submission, I had the opportunity of spending 6 weeks collaborating at ONERA (Meudon, France), evaluating and sharing my research on sensitivity analysis.

For readability, this thesis is therefore not written in chronological order, but divided in topics by chapters to provide a better contents structure. I decided to group the three publications related with the Purdue University collaboration in a long chapter of the thesis, where it is easier to follow the development of the research line. The results and theory on the research of sensitivity algorithms for stability analysis are also located in an individual part, with further description of the theoretical part and a specific section where the optimization algorithm is shown and evaluated.

There are other publications in which I participated on their elaboration on these three years[74, 146, 152], but are not included in this thesis as I am not the main author and they will appear on the corresponding PhD thesis or Journals. There were, however, excellent collaborations from which I could greatly expand my knowledge thanks to both, the main authors and the interesting topics. 


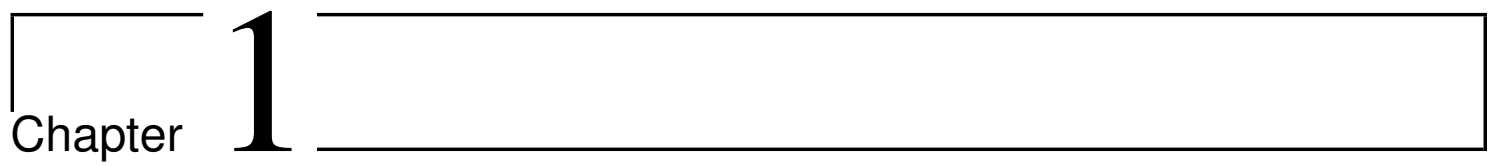

\section{Introduction}

It is not the process of

linearization that limits insight.

It is the nature of the state we

choose to linearize about.

E.T. Eady (attributed)

\subsection{Motivation}

Human kind has always been intrigued by complex flow phenomena. The behaviour of storms and their changing winds, the variable rhythm and direction of sea waves, the calm but complex flow of water on rivers and channels, or the convective currents generated on a wild fire are phenomena that, despite the differences, have something in common. Among others, those are events where slight variations of the flow temperature, density or pressure can have a great effect. Without falling into the beauty and simplicity of the so well-known (and so over-used) "butterfly effect", truth is that small perturbations on a fluid system may propagate in time and space, eventually driving the behaviour of the affected flow. If this is the case, we will be in the presence of a hydrodynamic instability.

We can find hydrodynamic instabilities in Nature, like the Rayleigh-Bénard convection[76], where buoyancy and gravity effects are responsible for the apparition of convection cells (Fig. 1.1-(a)), the Kelvin-Helmholtz shear instability, where the shear between two streams with different velocities destabilizes the flow generating a roll-up effect that creates a sequence of crests (Fig. 1.1-(b)), or the contact line fingering instabilities[59], occurring when the interface of a liquid mass flowing down an inclined plate breaks into "fingers" (Fig. 1.1-(c)), among many others. All of them are easily observed and even related with the formation of solid patterns[43]. Hydrodynamic instabilities are also responsible of the laminar to turbulent flow transition, the phenomena involved on the evolution from an almost-unidirectional 


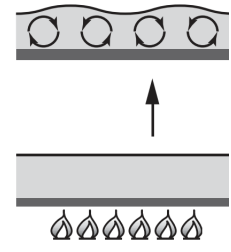

(a)

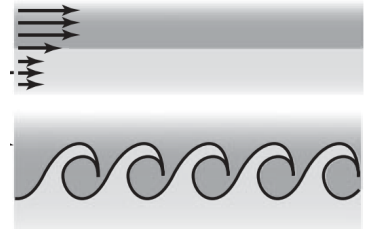

(b)

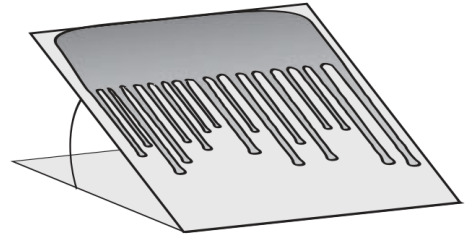

(c)

Figure 1.1: Sketch of common flow instabilities. Rayleigh-Bénard convection (a), Kelvin-Helmholtz shear instability (b) and contact line fingering instability (c).

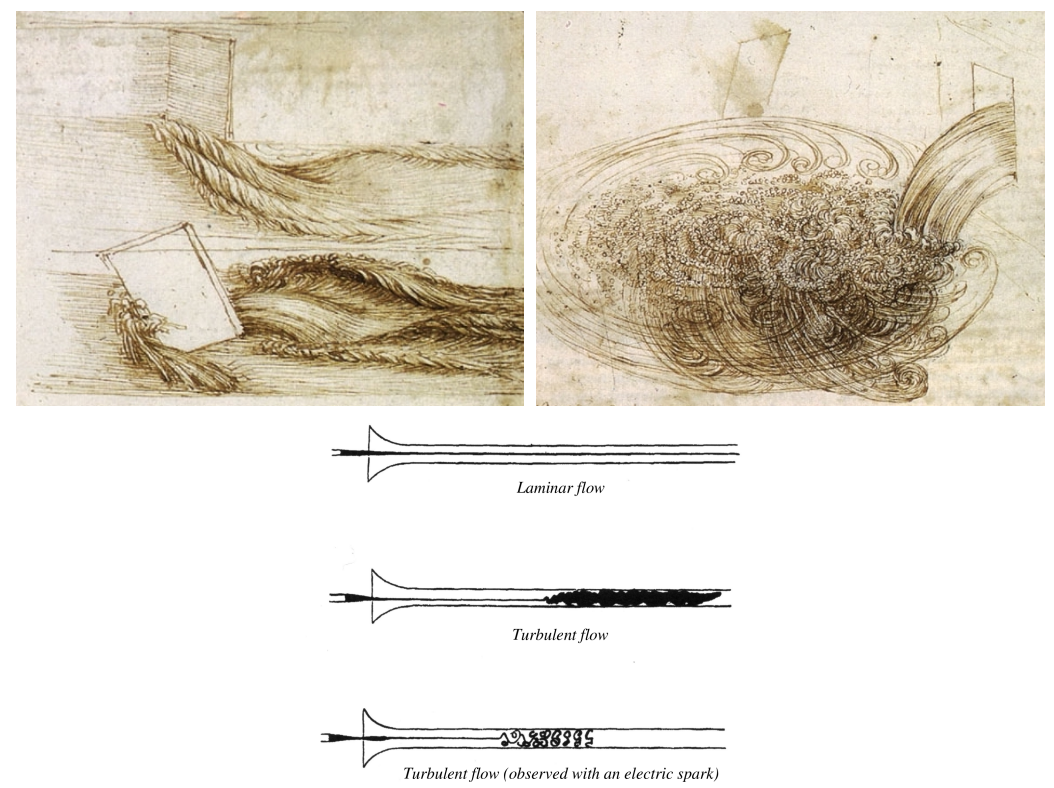

Figure 1.2: Da Vinci drawings on his studies of flow turbulence (left, centre). Figures from Reynolds on his analysis of turbulence transition (right).

flow brakes into multi-scale eddies with chaotic behaviour, enhancing the mixing and the dissipation. This particular behaviour was early observed by Leonardo da Vinci, with his impressive drawings and descriptions, and systematically studied and characterized by Osborne Reynolds[109] (Fig. 1.1). His work on the identification of the transition point on a pipe flow led to the definition of the Reynolds number, a dimensionless parameter that relates the viscosity of the fluid with its inertial forces, and that currently is one of the main parameters to classify the flow regime.

Nowadays, the analysis of the stability of a dynamic system permits to predict what kind of perturbations will trigger the apparition of undesired flow configurations, or even controlling them. Local stability analysis was first introduced for parallel flows, investigating simple boundary layer flows in the streamwise direction. In this type of analysis, only a portion of the flow configuration was investigated, extrapolating the results to model the stability behaviour of the whole flow field. 
Hence, its application was initially limited to parallel flows, but its results served to develop turbulence transition models as extended as the $e^{N}$ method[150]. Later on, local stability analysis found its way through the analysis of wake and shear flows[108, 111], where an assumption of weakly non-parallelism permits the use of this approach. The evolution of the computational resources enabled the possibility of obtaining a global stability analysis with no particular assumption of the flow configuration, opening the door to complex scenarios.

However, to recover the spatial structure of driving perturbations and their associated frequency/periodicity, a large eigenvalue problem has to be solved. The matrix forming and the analysis process require large amounts of memory, that can make the analysis not feasible, or at least not interesting outside of the academic world. The investigation of different methodologies to reduce these requirements, and the development of the numerical techniques to analyse industrial cases is therefore a rough but profitable path to take.

\subsection{Historical review and state of the art}

The flow behind a moving body is normally characterized by having low momentum and low pressure, due to the great separation and recirculation area created by viscous effects. The interaction among boundary layer, shear layer and wake makes these flows complex and difficult to investigate. Due to these difficulties, the wake behind a cylinder is often taken as a model of free shear wakes to study their dynamics and related instabilities $[44,137,156]$. The first and secondary instabilities of the cylinder wake were early investigated[12, 54, 97], and since then hundreds of publications have covered the different aspects of this particular flow. Currently, the analysis of the secondary instability of a three-dimensional sphere[130] and its control[45] involve a feasible numerical computation, while only 20 years ago it was only a remote possibility. But global analysis has not only given insight of the instability mechanisms of bluff body wakes, with wing wake flows also taking benefit from it. The analysis of a two-dimensional aerofoils of the NACA family serves to identify instabilities related with massive separation at high angles of attack[67], the vortex shedding phenomena that develops behind a lifting body at high angles of attack[151], or the receptivity characteristics of laminar separation bubbles on aerofoils[64]. A recent contribution between experimental, numerical and stability analysis results on the analysis of trailing vortices of finite aspect ratio wings[53] is establishing global stability analysis as the third pillar on complex flows characterization, together with Computational Fluid Dynamics (CFD) and experimental analyses.

On the aerospace field, the study of hydrodynamic instabilities is growing roots as a tool to explain and control complex flow phenomena. For high speed flows, the instability related with the shock-wave boundary layer interaction has been widely studied in recent years. From the modelling of the impingement of 
shock waves on boundary layer flows[50, 110, 124], the study of non-steady separations related with the buffet phenomenon[26, 27, 61, 123] (event recent contributions for 3D configurations[147]) is of great interest due to, non only the limits on aircraft performance that buffet phenomenon imposes, but the oscillating loads generated by buffeting that can be very detrimental to the aircraft lifespan[63, 70]. Also of interest is the investigation of global instabilities on axisymmetric wakes, due to its direct relation with the flow behind slender bodies. On this area, it is remarkable the research on supersonic turbulent wakes[117, 118] and the advances in flow control of afterbody flows[87]. Global analysis has been used so far in a wide range of applications, and it is still expanding $[139,144]$. The extension of stability analysis goes far beyond, including heat-transfer phenomena[84], acoustic resonance in cavities[28, 40, 145], mixing of fuel and air in jet combustors or swirling flows [98, 142], among many other examples[7, 23, 65].

Of particular interest is the study of the adjoint eigenvalue problem (the original being named as direct problem), which applications are currently far beyond those originally imagined. On the study of linear systems, sometimes is interesting to know their response to a change of specific input variables. If the number of output variables is greater to the number of inputs, solving again the linear system for those changes is effective. However, if the number of inputs increase, it might be more effective to reformulate the system computing the adjoint problem of the system, allowing to obtain also the sensitivities of the output to input changes. The stability analysis of the adjoint linear operator of the Navier-Stokes provides its sensitivity to changes in the initial and boundary conditions[126], volume forces or mass injection rate[46]. Moreover, the adjoint field is also a powerful tool to investigate the receptivity or the transient growth of the system[125], also being effective on the application of flow control[75]. The adjoint global modes can be interpreted as the initial condition that has maximum projection along the direction of the corresponding direct eigenmode[36]. They contain information about the instability that cannot be inferred from a simple analysis of the results of the direct problem. Furthermore, the combination of the two, adjoint and direct eigenmodes, provides the so-called structural sensitivity, a gradient that quantifies the drift of the associated eigenvalue when a structural change is introduced in the base flow, and identifies the wavemaker areas, core of the instability[46]. The beauty of this formulation is that, the identification of the flow regions more sensitive to hydrodynamic instability, can be applied to any kind of flow regime and complexity, from incompressible bounded flows[37] to high supersonic flows[96]. The use of adjoint methods has also proven effective to obtain the sensitivity of the eigenvalue to certain inputs, as changes in the base flow[16] or the introduction of a steady forcing[77]. First calculated for the leading instability of the laminar cylinder wake flow[77, 89], this methodology was quickly extended to more complex configurations[3, 39, 40, 86, 123], and even applied to secondary instabilities[24, 45]. The further development of adjoint methodologies has been extended to flow actuation[18, 141] and wall deformation[17, 140]. It is rare nowadays the stability analysis that does not contemplate an adjoint approach[75]. 

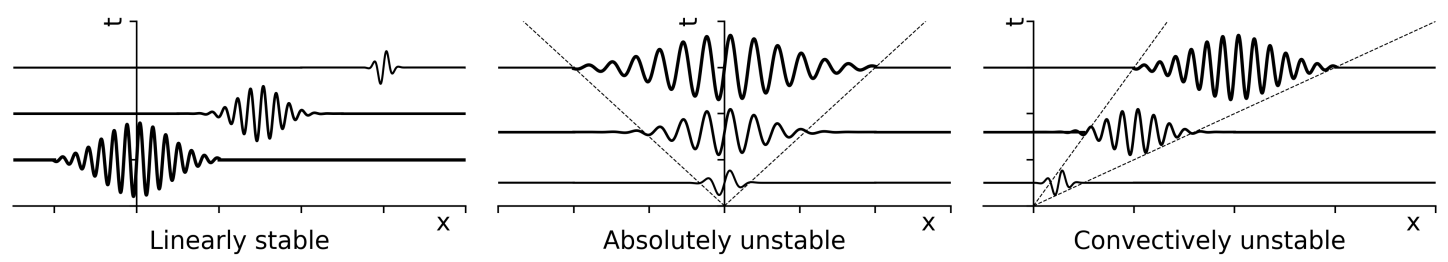

Figure 1.3: Types of asymptotic instability

Moreover, the study of the adjoint problem associated to the stability analysis can be used to obtain relevant information related to the hydrodynamic instability. Optimal forcing, receptivity, or stabilizing/destabilizing a certain flow configuration are, among others, properties that can be extracted from and adjoint analysis. A combination of global stability analysis, adjoint methods and sensitivity studies can provide useful information to design and flow control engineers.

\subsection{Linear stability analysis}

To identify the critical conditions for which the instability develops is of major interest, as a better understanding of the phenomenon will avoid undesirable transitions. These conditions are normally marked by a "tipping" point, when qualitative changes appear and a new flow configuration develops. Using bifurcation theory we can track and identify the critical conditions, while studying the asymptotic behaviour of the flow. For this, we briefly revisit the concept of stability, normally explained through the concept of Lyapunov stability. Starting from an equilibrium state $G(x, 0)$ (fixed point of our non-linear dynamical system, namely the NavierStokes equations), the system is perturbed around it. If the system is stable, the perturbation decays in time and small variations at the starting point will alter only the system at some future time.

$$
\left\|G(x, 0)-G^{*}(x, 0)\right\|<\delta \rightarrow\left\|G(x, t)-G^{*}(x, t)\right\|<\epsilon
$$

In the concept of Lyapunov stability we consider asymptotic instability, with an infinite time horizon. So, a system will be stable if, after perturbed, it eventually recovers its initial state:

$$
\left\|G(x, 0)-G^{*}(x, 0)\right\| \rightarrow 0, \text { as } t \rightarrow \infty .
$$

If the perturbation decays in time and in every direction, the flow is stable. If the perturbation grows downstream but decays at its origin, the flow is convectively unstable. Finally, if the perturbation grows at the point of impulse the flow is classified as absolutely unstable (Fig. 1.3)

Our interest lies on the time evolution of small disturbances from the equilibrium state, and how they affect the asymptotic behaviour and the states reached 
by the system, the so-called attractors of the model. The simplest attractors of a model are fixed points, also called steady states of the flow (base flows), followed by periodic orbits (oscillators), quasi-periodic behaviours and chaotic attractors (the last two not covered on this thesis). Of main interest for us are then the Pitchfork bifurcations, where the stability of the system is transferred from a symmetric solution to a pair of steady conjugated solutions coexisting two stable and one unstable attractors (i.e. a channel flow passing through a sudden expansion/contraction[34], where the flow losses its symmetry with respect to the mid plane), and Hopf bifurcations, where the system transfers from a steady state solution to a limit cycle (i.e. the onset of the vortex shedding of the cylinder wake[137]). Shear and parallel flows can act as a filter (also called noise amplifier) where perturbations are amplified/damped in space, or show a characteristic dynamic where the behaviour of the flow is led by specific frequencies[58]. In the same way as an oscillator, they may show internal self-excited resonance when the absolute instability develops, being defined by a characteristic frequency. Our interest is on spatially developing flows, where the perturbations (or oscillations in case of time periodic flows) extend over large regions of the flow. The spatial structure of the perturbations is then referred as "global mode". These modes can be obtained and analysed from a linear perspective, using Modal Stability Theory[65, 144]. Modal analysis relies on the decomposition of any flow quantity into a steady part $\overline{\mathbf{q}}$ (referred as base flow) and an unsteady part $\tilde{\mathbf{q}}$, namely the small perturbations of the flow:

$$
\mathbf{q}(\mathbf{x}, t)=\overline{\mathbf{q}}(\mathbf{x})+\varepsilon \tilde{\mathbf{q}}(\mathbf{x}, t)
$$

The perturbations are assumed to be of the form $\tilde{\mathbf{q}}=\hat{\mathbf{q}} \exp \{i \Phi\}$, where $\hat{\mathbf{q}}$ and $\Phi$ are the spatial structure and phase functions of the perturbation, respectively. Introducing these solutions into the linearised version of the Navier-Stokes equations, and considering $\Phi=\sigma t$, gives rise to an eigenvalue problem with eigenvalues $\left\{\sigma_{i}\right\}$. The eigenvectors $\hat{\mathbf{q}}$, solutions of the eigenvalue problem, are the global modes of the system, which for an unstable $\sigma$ will impose their dynamics to the flow.

\subsection{Thesis objectives and organization}

The eigenvalue problem associated to the Linear Navier-Stokes (LNS) problem is not trivial to solve. The condition number of the associated matrices (ratio between the smallest ant the largest eigenvalues) is normally very high, and the dimensions of the linear operator are very large due to the high spatial resolution needed to obtain an accurate flow solution. The use of Krylov subspace methods to reduce the dimension of the problem allows to recover only the part of the eigenvalue spectrum of interest, but a calculation of the inverse of the operator is still required. Widely extended, a Lower-Upper (LU) decomposition is normally applied to the sparse operator for an easier computation of the Krylov subspace. However, the resulting Lower (L) and Upper (U) matrices of the decomposition can be less sparse 
than the initial operator, making this type of analysis difficult to afford due to the associated memory requirements. This problem becomes more important as the Reynolds number increases, or non-linearities appear in the flow domain. Hence, we propose the use of simplified physical models, combined with the calculation of the stability characteristics of only part of the domain (still from a global approach, but neglecting the influence of regions far from the perturbations) for the evaluation of high Reynolds and turbulent flows. Our interest is therefore in developing optimal strategies for conducting stability analysis of turbulent and complex flows with a high Mach number.

To evaluate the influence on the global instability of external factors, such as base flow modifications or the presence of forcing, it is possible to calculate the sensitivity of the eigenvalue to these changes. When the instability is related with the presence of a body (or affected by it), the modification of the body shape could alter the onset of the instability or the associated frequency of its oscillations. However, the information gathered from the existent methodologies to compute the eigenvalue sensitivity analysis may be incomplete to guide a shape optimisation process. It would be of interest then to develop the mathematical formulation to evaluate the sensitivity to changes in a specific geometrical parameter, or the body surface shape.

The present thesis is organized in the following chapters as follows:

- Chapter 2 presents the mathematical formulation upon which the stability and sensitivity analysis are performed. The non-linear compressible Navier-Stokes equations are linearised around a point of equilibrium, and modal solutions of the obtained linearised problem are proposed. The properties of the resultant linear operator, the Jacobian of the flow solution, govern the stability of the system. The linearised problem can be recasted into a generalised eigenvalue problem, which eigenvectors are the "global modes" of the system, with its stability properties controlled by their associated eigenvalues. An introduction to adjoint methods in hydrodynamic stability is included, to present the concept of the eigenvalue sensitivity. A Lagrangian approach is considered to extract the sensitivity of the eigenvalue associated to a global mode of interest to different perturbations, such as base flow modifications or steady forcing.

- Chapter 3 describes the numerical implementation of the theoretical approach. An introduction to the flow solver used on the calculation of the states of equilibrium of the Navier-Stokes equation is included, together with the description of the construction of the Jacobian matrix of the flow solution. A discrete approach is used, where the flow solution is first discretised and then linearised. This methodology permits the reduction of the size of the associated eigenvalue problem using a subspace projection where only part of the linear operator is used on the final eigenvalue problem, without having troubles with artificial boundary conditions. The implementation of the sensitivity 
calculation is discussed, as the discrete approach demands some manipulation of the linear operators to extract the different eigenvalue sensitivities. Finite differences are used to compute the second derivatives of the flow solution, by perturbing the Jacobian matrix on the regions of interest.

- Chapter 4 is dedicated to the validation of the theory and methodology presented in Chapters 1 and 2. Two laminar and two turbulent flows are considered, with their results being compared with similar cases in the literature. The low speed flow over an infinite circular cylinder is revisited to extract the characteristic global mode related with the cylinder wake first instability. A Hopf bifurcation that breaks the symmetry of the wake, generating downstream vortices and increasing the aerodynamic drag of the cylinder. The evaluating the eigenvalue sensitivity complex fields reports not only those region more sensitive to steady forcing, but also where the placement of a small control body can delay the vortex shedding phenomenon apparition. A channel sudden expansion is used as a second laminar flow validation example, where a Pitchfork bifurcation generates two possible steady fixed points on which the flow is no longer symmetric with respect to the mid plane. Turbulent scenarios are examined via the D-shaped cylinder, whose turbulent wake has a dominant global mode. This last case acts as a benchmark configuration for mean flow and turbulent analyses, proving the validity of the methodology that will be later applied in Chapter 6 .

- Chapter 5, containing the extensive parts of the results of this thesis, focuses on the application on direct and adjoint methods to an industrial case: a base bleed actuation over a turbine blade trailing edge. Unexpected non-symmetric flow configurations may appear at the base region of the aerofoil when cooling flow used to refrigerate the blade is ejected at the trailing edge. In this Chapter, we demonstrate that the break of symmetry comes from the destabilization of a global mode related with the base bleed injection channel. First, the instability is explored on supersonic regime, explaining literature results by means of stability analysis. These results are then expanded to subsonic, transonic and higher supersonic regimes in the following section, identifying the instability and exploring the influence of the trailing edge geometry on its development. The analyses are completed with the extraction of the sensitivity gradients of the leading global mode for each of the sonic regimes.

- Chapter 6 contains the application of the eigenvalue sensitivity to geometrical changes to a shape optimization case. The methodology to obtain the sensitivity of the eigenvalue to geometry changes for each singular mesh node is introduced in a non-expensive way, easing the calculation of the gradients by just one single extra computation. The gradients are obtained for a laminar flow around an infinite two dimensional cylinder, at Reynolds number value of 60. Information for each mesh node is extracted, showing a direct relation 
between the expected changes on the amplification rate or the frequency of the eigenvalue when deformations are applied. As a test case, the first instability of the cylinder wake is controlled by surface deformation, using the information relative to each mesh node to guide mesh node displacements towards the minimization of the cost function. Two different scenarios are shown, considering as the cost function either the eigenvalue amplification rate (real part) or the associated frequency (imaginary part).

\subsection{Novelty of the work and main contributions}

During this thesis, several analyses of high-speed complex flow configurations using linear stability are completed, identifying the physical mechanisms that drive a global instability occurring due to the ejection of cooling flow at the trailing edge of a turbine blade, and correlating the results with CFD calculations. A complete sensitivity analysis of the unstable eigenvalue provides information with respect to how changes in the flow configuration or the presence of forcing would affect the behaviour of the instability. The study is then expanded to further sonic regimes, as part of a combined numerical and experimental campaign, confirming the presence of the global mode in different base bleed configurations. The stability analysis detected the onset and decay of physical phenomena on a supersonic flow configuration, that had been previously documented in the literature by experimental and numerical means.

In addition, a simple and with negligible computational cost framework to extract the eigenvalue sensitivity to changes in a body surface has been developed. The methodology appears as an additional step after the calculation of the sensitivity to steady forcing, which is easily evaluated in the discrete framework here contemplated. Moreover, the obtained gradients have shown excellent performance on the control of a well known global instability, as the onset of the vortex shedding of a cylinder wake.

\section{List of publications}

\section{Journal publications}

- Martinez-Cava, A., Wang, Y., Vicente, J. de, and Valero, E., "Pressure Bifurcation Phenomenon on Supersonic Blowing Trailing Edges", AIAA Journal, vol. 57, Jan. 2019, pp. 153-164.

- Martinez-Cava, A., Vicente, J. de, Ferrer, E. and Valero, E., "Eigenvalue Sensitivity to Surface Geometry Modifications", 2019. To be submitted for publication. 
- Wang, Y., Ferrer, E., Martinez-Cava, A., Zheng, Y., and Valero, E., "Enhanced stability of flows through contraction channels: combining shape optimisation and linear stability analysis", Physics of Fluids, vol. 31:7, 2019.

\section{Conference proceedings}

- Martinez-Cava, A., Valero, E., and Vicente, J. De, "Sensitivity analysis of supersonic turbine trailing edges", Proceedings of 7th European Conference on Computational Fluid Dynamics (ECFD 7), Glasgow, UK, 2018, pp. 502-518.

- Wang, Y., Martinez-Cava, A., Valero, E., Zheng, Y., and Ferrer, E., "Eigenmode Optimization of a Contraction Channel Based on Stability Analysis", Proceedings of 7th European Conference on Computational Fluid Dynamics (ECFD 7), Glasgow, UK, 2018, 2018, pp. 4299-4310.

- Martinez-Cava, A., Valero, E., de Vicente, J., and Paniagua, G., "Coanda Flow Characterization on Base Bleed Configurations Using Global Stability Analysis", AIAA Scitech 2019 Forum, San Diego, California, 2019.

- Martinez-Cava, A., Valero, E., Saavedra, J., Lozano, F., and Paniagua, G., "Optimization of the base bleed to control trailing edge flows in a wide range of Mach numbers", Proceedings of the 8th European Conference for Aeronautics and Space Sciences (EUCASS), Madrid, Spain, 2019.

- Lozano, F., Saavedra, J., Paniagua, G., Martinez-Cava, A., and Valero, E., "Experimental Approach to Characterize Base Region Coanda Flow Topology Induced by Pulsating Blowing", Proceedings of the 8th European Conference for Aeronautics and Space Sciences (EUCASS), Madrid, Spain, 2019. 


\section{Part I}

\section{Theoretical Description \& Numerical Implementation}





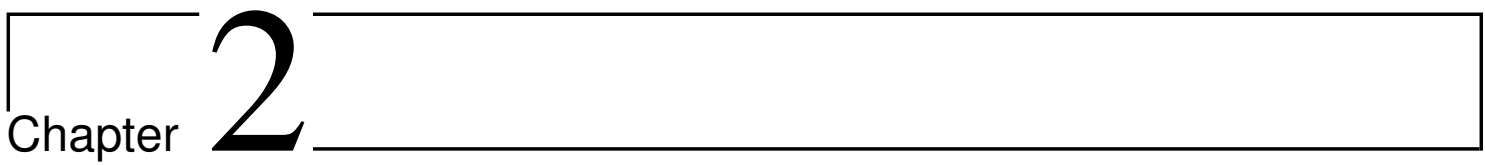

\section{Mathematical Development}

\section{Contents}

2.1 Global Stability Analysis . . . . . . . . . . 15

2.1.1 Definition of an adjoint eigenvalue problem . . . . . . 16

2.1.2 Turbulence analysis validity and mean flow analysis . . . 19

2.2 Eigenvalue Sensitivity Analysis ........... . 21

2.2.1 Sensitivity to base flow modifications . . . . . . . . 21

2.2 .2 Sensitivity to external forcing . . . . . . . . . . . 23

\subsection{Global Stability Analysis}

We consider the non-linear system of PDEs defined by the compressible version of the Reynolds Averaged Navier-Stokes (RANS) equations and the imposed boundary conditions, written in conservative form:

$$
\frac{\partial \mathbf{q}}{\partial t}=\mathbf{R}(\mathbf{q})
$$

where vector ${ }^{1} \mathbf{q}$ is the state vector that comprises the conservative flow variables, and $\mathbf{R}(\mathbf{q})$ is the discrete spatial non-linear operator, comprising the inviscid and viscous terms. Without loss of generality, we decompose the solutions of Equation (2.1) into a steady component upon which a small perturbation is superimposed:

$$
\mathbf{q}(\mathbf{x}, t)=\overline{\mathbf{q}}(\mathbf{x})+\varepsilon \tilde{\mathbf{q}}(\mathbf{x}, t)
$$

where $\varepsilon \ll 1$ and $\overline{\mathbf{q}}$ is the so called base flow, steady point of equilibrium of Equation (2.1).

\footnotetext{
${ }^{1}$ Bold variables are used to refer vectors.
} 
This decomposition is introduced into System (2.1) and a Taylor series expansion is performed around the point of equilibrium $\overline{\mathbf{q}}$. Neglecting terms of order $\varepsilon^{2}$ and assembling all the unknowns in vector/matrix notation, we obtain:

$$
\varepsilon \mathbf{M} \frac{\partial \tilde{\mathbf{q}}}{\partial t}=\mathbf{R}(\overline{\mathbf{q}}+\varepsilon \tilde{\mathbf{q}}) \approx \mathbf{R}(\overline{\mathbf{q}})+\varepsilon\left[\frac{\partial \mathbf{R}(\mathbf{q})}{\partial \mathbf{q}}\right]_{\mathbf{q}=\overline{\mathbf{q}}} \tilde{\mathbf{q}},
$$

where $\mathbf{M}$ is the mass matrix resulting from the spatial discretisation and $[\partial \mathbf{R}(\mathbf{q}) / \partial \mathbf{q}]_{\overline{\mathbf{q}}}$ represents the Jacobian matrix of the fluxes evaluated for the base flow, which properties govern the stability of the system. On a finite volume approximation (see Section 3.1), the mass matrix $\mathbf{M}$ is diagonal with leading dimension $N_{v} \times N$ and contains the volumes associated to each cell.

In this thesis, perturbations are assumed to be two dimensional and of the form

$$
\tilde{\mathbf{q}}(\mathbf{x}, t)=\hat{\mathbf{q}}(x, z) \exp \{\sigma t\}+c . c .
$$

where $\sigma$ is a complex scalar, $\hat{\mathbf{q}}$ describes the complex disturbance function of $x$ and $z$, and c.c. stands for complex conjugate. Complex conjugation is included since the pair $(\hat{\mathbf{q}}(x, z))$ and its complex conjugate are both solutions of the linearised NavierStokes equations, while $\tilde{\mathbf{q}}$ is real, and the phase function of the normal modes has been rotated for simplicity as $-i \sigma \rightarrow \sigma$. Introducing Equation (2.4) in (2.3), and considering $\mathbf{R}(\overline{\mathbf{q}})=0$, the last is recasted into the generalized eigenvalue problem (GEVP):

$$
\mathbf{J}(\overline{\mathbf{q}}) \hat{\mathbf{q}}=\sigma \mathbf{M} \hat{\mathbf{q}},
$$

with the linear operator $\mathbf{J}(\overline{\mathbf{q}})=[\partial \mathbf{R}(\mathbf{q}) / \partial \mathbf{q}]_{\overline{\mathbf{q}}}$ being the Jacobian matrix with leading dimension $N \times N_{v}$, and $\sigma=\sigma_{r}+i \sigma_{i}$ the complex eigenvalue of the generalized eigenvalue problem. The real part of the eigenvalue, $\sigma_{r}$, refers to the amplification rate of the corresponding eigenmode, with the imaginary part $\sigma_{i}$ being the pulsation, related to the associated Strouhal number as $S t=\sigma_{i} / 2 \pi$. For simplicity, system (2.5) is rewritten as a standard eigenvalue problem (EVP) through the operator $\mathbf{A}=\mathbf{M}^{-1} \mathbf{J}$ :

$$
\mathbf{A}(\overline{\mathbf{q}}) \hat{\mathbf{q}}=\sigma \hat{\mathbf{q}}
$$

\subsubsection{Definition of an adjoint eigenvalue problem}

Adjoint approaches are a common topic on optimization and stability analysis but, before extending into the associated adjoint formulation of our stability problem, let us introduce the need of an adjoint approach.

If a discrete linear system is considered, with finite number of variables that depend linearly on a finite number of others in an instantaneous way, the vector of state can be defined by its explicit relation with an input by

$$
\mathbf{x}=\mathbf{C u}
$$


with $\mathbf{x}$ being a real or complex array of size $M, \mathbf{u}$ a vector of size $N$ and the operator C a $M \times N$ matrix. If one is interested in a linear function of $\mathbf{x}, W$, expressed as

$$
W=\mathbf{y x},
$$

with $\mathbf{y}$ a vector of size $M$, it would be necessary to evaluate Equation (2.7) followed by (2.8) for each input $\mathbf{u}$. However, introducing (2.7) into (2.8) one recovers $\mathbf{y x}=$ $\mathbf{y C u}=\mathbf{p u}$, with $\mathbf{p}=\mathbf{y C}$, which can also be written as

$$
\mathbf{v}=\mathbf{C}^{T} \mathbf{y} .
$$

Writing our linear function $\Omega$ in terms of vector $\mathbf{v}$, it remains

$$
W=\mathbf{v u} .
$$

Despite its simplicity, this formulation permits to calculate vector $\mathbf{v}$ from $\mathbf{y}$ just once, and then evaluate $W$ for different values of $\mathbf{u}$. Vector $\mathbf{v}$, here defined as the derivative of our objective linear function $W$, is the sensitivity of the objective to the input. Equation 2.9 is the so-called adjoint problem of Equation 2.7. If the number of outputs of interest, namely our linear function $W$, is higher than the number of inputs, the standard approach of solving Equation (2.7) followed by (2.8) can be followed. In contrast, if the number of inputs exceeds the number of outputs, the formulation of the adjoint problem results more efficient. Moreover, evaluating the sensitivity of the objective linear function to different inputs can be an asset on evaluating/optimizing the system.

Extending this formulation to a system of linear algebraic equations, where vectors $\mathbf{x}$ and $\mathbf{u}$ are implicitly related by a non-singular operator:

$$
\mathbf{H x}=\mathbf{u},
$$

where $\mathbf{H}$ is a non-singular square matrix of size $M \times M$. The shortest way to obtain the adjoint problem will be transforming Equation 2.11 into 2.8 and then proceed as described above. However, calculating the inverse of $\mathbf{H}$ might not be possible, and a more efficient alternative will be to write the objective function $W$ as in Equation (2.10):

$$
W=\mathbf{v u}=\mathbf{v H x}=\mathbf{y} \mathbf{x} .
$$

This becomes an identity of any input $\mathbf{u}$ if

$$
\mathbf{H}^{T} \mathbf{v}=\mathbf{y}
$$

namely the adjoint system of (2.11).

On a Navier-Stokes context, the linear operator $\mathbf{A}(\mathbf{q})$ is normally ill-conditioned and non-normal, which induces a large sensitivity of the spectrum to perturbations or forcing[23]. Therefore, an adjoint approach plays a major role on the definition and identification of these sensitivities. 
The adjoint problem of (2.5) is hence defined as

$$
\mathbf{A}^{+} \hat{\mathbf{q}}^{+}=\sigma^{+} \hat{\mathbf{q}}^{+}
$$

with $\hat{\mathbf{q}}^{+}$as the adjoint eigenvectors and $\sigma^{+}$the eigenvalues of the adjoint problem. The eigenvalues of the adjoint problem are complex conjugate of those of the original system[57]. To relate it with the eigenvalue problem (EVP) obtained in (2.6) in the discrete framework of the finite volume discretisation, the following discrete inner product of a pair of arbitrary vectors, $\mathbf{c}$ and $\mathbf{d}$, is defined as

$$
<\mathbf{c}, \mathbf{d}>=\int_{\Omega} \mathbf{c}^{H} \mathbf{d} d \Omega=\mathbf{c}^{H} \mathbf{M d}
$$

where the superindex $H$ denotes the conjugate transpose and $\mathbf{M}$ is the volumes matrix previously defined.

System (2.14) allows to obtain the adjoint eigenvalues and eigenmodes of the formulated eigenvalue problem. However, for the calculation of further analyses, which imply the multiplication of the eigenmodes with the linearised Jacobian matrix (among other operators), one would like to recover the adjoint eigenmodes associated to the weighted inner product to preserve the integrity of the solution. With this aim, we redefine here the adjoint operator using the defined inner product and relate the adjoint eigenmodes of the system with those obtained with the new operator.

The previously defined inner product also provides a definition of the adjoint operator $\mathbf{A}^{+}$as $<\mathbf{c}, \mathbf{A d}>=<\mathbf{A}^{+} \mathbf{c}, \mathbf{d}>$. From this definition, it can be derived:

$$
\begin{gathered}
<\mathbf{c}, \mathbf{A d}>=\mathbf{c}^{H} \mathbf{M A d}=\mathbf{c}^{H} \mathbf{M A} \mathbf{M}^{-1} \mathbf{M d}= \\
\left(\mathbf{M}^{-1} \mathbf{A}^{H} \mathbf{M}\right)^{H} \mathbf{c}^{H} \mathbf{M d}=<\left(\mathbf{M}^{-1} \mathbf{A}^{H} \mathbf{M}^{-1}\right) \mathbf{c}, \mathbf{d}>
\end{gathered}
$$

arriving to

$$
\mathbf{M}^{-1} \mathbf{A}^{H} \mathbf{M}=\mathbf{A}^{+}
$$

Expression (2.17) relates the conjugate transpose of the Jacobian matrix to its adjoint operator, allowing to obtain the associated adjoint eigenvalues, $\sigma^{+}$, and eigenmodes, $\hat{\mathbf{q}}^{+}$. Substituting the value of $\mathbf{A}^{+}$on Equation (2.14) we arrive to the definition of the discrete adjoint eigenvalue problem:

$$
\mathbf{M}^{-1} \mathbf{A}^{H} \mathbf{M} \hat{\mathbf{q}}^{+}=\sigma^{+} \hat{\mathbf{q}}^{+}
$$

which can be solved as $\mathbf{A}^{H} \mathbf{p}=\sigma^{+} \mathbf{p}$, recovering the adjoint eigenmodes with $\hat{\mathbf{q}}^{+}=$ $\mathbf{M}^{-1} \mathbf{p}$.

To conclude, if we consider the eigenpairs $\left(\sigma_{j}, \hat{\mathbf{q}}_{j}\right)$ and $\left(\sigma_{i}^{+}, \hat{\mathbf{q}}_{i}^{+}\right)$of matrix $\mathbf{A}$ and its adjoint, starting with the identity defined by multiplying the adjoint problem, 
$\left(\sigma_{i}^{+}-\mathbf{A}^{+}\right) \hat{\mathbf{q}}_{i}^{+}=0$, by the eigenvector $\hat{\mathbf{q}}$ we can write:

$$
\begin{aligned}
&<\left(\sigma_{i}^{+}-\mathbf{A}^{+}\right) \hat{\mathbf{q}}_{i}^{+}, \hat{\mathbf{q}}_{j}>=0 \\
&<\hat{\mathbf{q}}_{i}^{+},\left(\sigma_{i}-\mathbf{A}\right) \hat{\mathbf{q}}_{j}>=0 \\
&<\hat{\mathbf{q}}_{i}^{+},\left(\sigma_{i}-\mathbf{A}-\sigma_{j}+\mathbf{A}\right) \hat{\mathbf{q}}_{j}>=0 \\
&<\hat{\mathbf{q}}_{i}^{+},\left(\sigma_{i}-\sigma_{j}\right) \hat{\mathbf{q}}_{j}>=0 \\
&\left(\sigma_{i}-\sigma_{j}\right)<\hat{\mathbf{q}}_{i}^{+}, \hat{\mathbf{q}}_{j}>=0
\end{aligned}
$$

where in Equation (2.22) we have added the equality of the direct problem $\left(\sigma_{j}-\right.$ A) $\hat{\mathbf{q}}_{j}=0$. Expression (2.23) is the so-called bi-orthogonality condition, relating the eigenvectors of the direct and adjoint matrix as orthogonal to each other, if they are not associated with the same eigenvalue.

\subsubsection{Turbulence analysis validity and mean flow analysis}

The stability analysis requires the consideration of a base flow, which is taken as reference for the linearisation. Standard stability analysis normally considers a "steady base flow" and, in fact, "pure" linearisation theory requires this base flow to be solution of the steady or unsteady Navier-Stokes equations. In order to obtain steady base flows, different approaches can be followed. The most usual is finding a steady (not necessarily stable) solution of the Navier-Stokes equations. If the solution is unstable, continuation, Newton-Raphson or frequency damping methods are necessary to artificially obtain the desired base flow. When RANS (steady) simulations are considered on the calculation of the base flow, being these used as a reference for the linearisation of the non-linear operator, these steady solutions satisfy the Navier-Stokes equations and the considered turbulence model.

It may not be immediately obvious that a linear decomposition can be used to analyse a base flow obtained by a RANS calculation. The approximation is based on the classic triple-decomposition of Hussain and Reynolds[60]. Namely, the flow is decomposed into a time-mean part, an unsteady/periodic part, and a randomly fluctuating (turbulent) part. The mean is obtained from time-averaging, following usual RANS approach. Small-scale turbulent fluctuations are modelled using a turbulence model. A non-steady or coherent part of the flow can also be present and could be solved using URANS computations. This separation of scales allows for the treatment of the large coherent structures independently from the random turbulent fluctuations.

The stability analysis of unsteady base flows may requires the use of Floquet theory, which is not in the scope of this work. The use of mean flows, obtained by time averaging of a URANS solution (the turbulent fluctuations are already modelled), despite the differences, permits to obtain useful information from their analysis, sometimes with better accuracy than other approaches[11]. In fact, mean flow analysis can be very useful on the analysis of experimental data[98]. 
Going back to our system of non-linear PDEs, Equation (2.1), we had considered so far that the flow solutions belonged to a steady point of equilibrium of the system, $\mathbf{R}(\overline{\mathbf{q}})=0$, with $\overline{\mathbf{q}}$ being denoted as the already mentioned base flow. However, if the flow solution is non-stationary and periodic, with $\mathbf{q}(\mathbf{x}, t)=$ $\mathbf{q}(\mathbf{x}, t+T)$, a mean flow $\overline{\mathbf{q}}_{m}$ can be obtained by averaging the unsteady RANS (URANS) solution[11]. It has been proved by several authors that a linear criterion applied to the mean flow provides good results for the prediction of the frequency of the unsteadiness $[11,66,98,105]$. The conditions of validity for this analysis are well described in [132], where it was shown that the mean flow has to be approximately marginally stable, and the frequency of the limit cycle equal (or very close) to the frequency of the mean flow. These conditions are normally well satisfied on wake flows, but cannot be fulfilled by certain enclosed or semi-enclosed flows. This is also well related in Ref.[142]:

"(...) the approach of mean flow stability (...) relies on the following three assumptions:

(i) the harmonics have a much smaller amplitude than the fundamental mode(s) under investigation;

(ii) no other modes or their products are harmonically related to the dominant mode(s) under investigation (or, if they are harmonically related, the total amplitude of such modes remains an order of magnitude weaker than the dominant mode(s));

(iii) the eddy-viscosity hypothesis is appropriate for modelling of the remaining turbulent fluctuations."

However, both analyses (base flow and mean flow) coincide at the bifurcation point so, for solutions near critical flow values, both cases are equally valid[132].

Respect to the non-linear effects present in non-steady flows, Ref.[142] also establishes:

"The mean flow stability analysis does not assume that nonlinear interactions between different eigenmodes and their harmonics have never happened in this flow (...). What we do assume is that the amplitudes of such nonlinear interactions are weak in the final flow field; they are of much lower amplitude than the dominant eigenmode and the chaotic turbulent fluctuations. If this holds true for the dominant limit cycle(s), then mean flow stability will approximate the frequency and mode shape of that limit cycle(s)."

In both considered cases, base flow and mean flow, the flow solution can be decomposed into a non-time-dependant term (steady or averaged) and small fluctuations fields as done in (2.2), computing the stability of the flow via the linear operator as described in section 2.1 . 
During this thesis, a base flow approach was mainly used. However, the calculation of complex flows sometimes required the use of a mean flow approach to obtain the physical eigenmodes of the system, as it will be shown in Section 4.2.1.

\section{$2.2 \quad$ Eigenvalue Sensitivity Analysis}

As already mentioned, stability analysis studies the growth or decay of perturbations superimposed onto a usually steady solution of the Navier-Stokes equations. The analysis can identify which particular features are prone to evolve under slight modifications of the flow conditions, either by introducing a perturbation or caused by a modification of some physical or geometrical parameters. The growth of these features would give rise to a completely different flow configuration. The stability of the system can be analysed solving the derived eigenvalue problem (Eq. 2.5), obtaining the relevant (physical) eigenmodes that would drive the system towards an unstable configuration.

Sensitivity analysis focuses instead on the response of the system to variations of certain parameters, either physical, geometrical or external perturbations, normally evaluated through the variation of an target functional. In our case, we are interested in the sensitivity of the eigenvalue associated to the particular eigenmode, normally unstable, and to assess how it will respond to the modification of chosen parameters or conditions of the flow configuration.

In this Section we revisit in detail the formulation given by Browne et al.[20] and Mettot et al.[89] for the calculation in a discrete framework of the sensitivity of the eigenvalue to changes in the base flow or steady forcing, formulated accordingly to the approach planteated in this thesis.

\subsubsection{Sensitivity to base flow modifications}

Once the eigenpair of eigenvalue and eigenvector related with the global mode are recovered, the calculation of the gradients is considered as an optimization problem, where the cost function is the eigenvalue itself. We need to impose our eigenvalue problem, together with other conditions, as the main constraints of the optimization. To do so, the problem is solved using the Lagrange Multipliers method. We therefore define the Lagrangian functional as

$$
\mathcal{L}\left(\overline{\mathbf{q}}, \sigma, \hat{\mathbf{q}}, \boldsymbol{\lambda}_{1}\right)=\sigma+<\boldsymbol{\lambda}_{1}, \mathbf{A}(\overline{\mathbf{q}}) \hat{\mathbf{q}}-\sigma \hat{\mathbf{q}}>,
$$

where it will be later confirmed that the Lagrange multiplier $\boldsymbol{\lambda}_{1}$ represents the adjoint eigenmode, $\hat{\mathbf{q}}^{+}$.

The calculation of the gradient of the Lagrangian gives rise to four different terms:

$$
\boldsymbol{\nabla} \mathcal{L}=<\frac{\partial \mathcal{L}}{\partial \overline{\mathbf{q}}}, \delta \overline{\mathbf{q}}>+<\frac{\partial \mathcal{L}}{\partial \sigma}, \delta \sigma>+<\frac{\partial \mathcal{L}}{\partial \hat{\mathbf{q}}}, \delta \hat{\mathbf{q}}>+<\frac{\partial \mathcal{L}}{\partial \boldsymbol{\lambda}_{1}}, \delta \boldsymbol{\lambda}_{1}>
$$


from which we would only want to retain the first term, as we are interested in the influence of the base flow on the eigenvalue.

Cancelling the gradient of the Lagrangian with respect to the Lagrange multiplier $\boldsymbol{\lambda}_{1}$ we recover the generalised eigenvalue problem:

$$
\begin{gathered}
<\frac{\partial \mathcal{L}}{\partial \boldsymbol{\lambda}_{1}}, \delta \boldsymbol{\lambda}_{1}>=<\delta \boldsymbol{\lambda}_{1}, \mathbf{A}(\overline{\mathbf{q}}) \hat{\mathbf{q}}-\sigma \hat{\mathbf{q}}>=0 \\
\mathbf{A}(\overline{\mathbf{q}}) \hat{\mathbf{q}}-\sigma \hat{\mathbf{q}}=0 .
\end{gathered}
$$

The derivation of the Lagrangian with respect to the eigenmode, $\hat{\mathbf{q}}$, provides the adjoint eigenvalue problem:

$$
\begin{aligned}
<\frac{\partial \mathcal{L}}{\partial \hat{\mathbf{q}}}, \delta \hat{\mathbf{q}}> & =<\boldsymbol{\lambda}_{1},(\mathbf{A}(\overline{\mathbf{q}})-\sigma) \delta \hat{\mathbf{q}}> \\
= & <(\mathbf{A}(\overline{\mathbf{q}})-\sigma)^{+} \boldsymbol{\lambda}_{1}, \delta \hat{\mathbf{q}}>=0 \\
& \mathbf{A}^{+}(\overline{\mathbf{q}}) \boldsymbol{\lambda}_{1}=\sigma^{+} \boldsymbol{\lambda}_{1},
\end{aligned}
$$

from which we can confirm that $\boldsymbol{\lambda}_{1}=\hat{\mathbf{q}}^{+}$. The derivation of the Lagrangian respect to the eigenvalue, $\sigma$, yields a normalization for the adjoint perturbation variables:

$$
\begin{aligned}
<\frac{\partial \mathcal{L}}{\partial \sigma}, \delta \sigma> & =\delta \sigma+<\boldsymbol{\lambda}_{1},-\delta \sigma \hat{\mathbf{q}}> \\
& =\delta \sigma-\delta \sigma<\boldsymbol{\lambda}_{1}, \hat{\mathbf{q}}>=0 \\
<\boldsymbol{\lambda}_{1}, \hat{\mathbf{q}}>= & <\hat{\mathbf{q}}^{+}, \hat{\mathbf{q}}>=\left(\hat{\mathbf{q}}^{+}\right)^{H} \mathbf{M} \hat{\mathbf{q}}=1 .
\end{aligned}
$$

Finally, the gradient of the Lagrangian with respect to the base flow leads to

$$
\begin{aligned}
<\frac{\partial \mathcal{L}}{\partial \overline{\mathbf{q}}}, \delta \overline{\mathbf{q}}> & =<\boldsymbol{\lambda}_{1}, \frac{\partial \mathbf{A}(\overline{\mathbf{q}}) \hat{\mathbf{q}}}{\partial \overline{\mathbf{q}}} \delta \overline{\mathbf{q}}> \\
& =<\left(\frac{\partial \mathbf{A}(\overline{\mathbf{q}}) \hat{\mathbf{q}}}{\partial \overline{\mathbf{q}}}\right)^{+} \boldsymbol{\lambda}_{1}, \delta \overline{\mathbf{q}}>.
\end{aligned}
$$

Assuming that the gradient of the Lagrangian and the gradient of $\sigma$ with respect to modifications in the base flow are equal, then:

$$
\Delta \sigma=<\nabla_{\overline{\mathbf{q}}} \sigma, \delta \overline{\mathbf{q}}>=<\frac{\partial \mathcal{L}}{\partial \overline{\mathbf{q}}}, \delta \overline{\mathbf{q}}>.
$$

Therefore, taking $\boldsymbol{\lambda}_{1}=\hat{\mathbf{q}}^{+}, \mathbf{B}(\overline{\mathbf{q}}, \hat{\mathbf{q}})=\partial(\mathbf{A}(\overline{\mathbf{q}}) \hat{\mathbf{q}}) / \partial \overline{\mathbf{q}}$ (from now on referred as the sensitivity matrix), expanding the inner products, and applying the adjoint operator previously defined, Equation (2.32) becomes:

$$
\boldsymbol{\nabla}_{\overline{\mathbf{q}}} \sigma=\mathbf{M}^{-1} \mathbf{B}^{H}(\overline{\mathbf{q}}, \hat{\mathbf{q}}) \mathbf{M} \hat{\mathbf{q}}^{+} \text {. }
$$

Matrix $\mathbf{B}(\overline{\mathbf{q}}, \hat{\mathbf{q}})$ is the Hessian matrix, derivative of the Jacobian with respect to the state vector, $\mathbf{q}$, or second derivative of the base flow. The solution of Equation 2.34 recovers the complex fields of the eigenvalue sensitivity with respect to changes in the base flow. 


\subsubsection{Sensitivity to external forcing}

External perturbations, modelled as volume source terms, can also be considered as constraints on the optimisation process. In this way, flow control techniques can be exploited and combined with the results of the stability analysis. To do so, we consider the application of a external force to the system in the form:

$$
\mathbf{R}(\overline{\mathbf{q}})=\mathbf{q}_{f} .
$$

This imposes an additional constraint in the Lagrangian form:

$$
\mathcal{L}\left(\overline{\mathbf{q}}, \sigma, \hat{\mathbf{q}}, \mathbf{q}_{f}, \boldsymbol{\lambda}_{1}, \boldsymbol{\lambda}_{2}\right)=\sigma+<\boldsymbol{\lambda}_{1}, \mathbf{A}(\overline{\mathbf{q}}) \hat{\mathbf{q}}-\sigma \hat{\mathbf{q}}>+<\boldsymbol{\lambda}_{2}, \mathbf{R}(\overline{\mathbf{q}})-\mathbf{q}_{f}>,
$$

where it will be later confirmed that the chosen multipliers, $\boldsymbol{\lambda}_{1}$ and $\boldsymbol{\lambda}_{2}$, relate to the adjoint eigenmode and the adjoint operator of the base flow, $\hat{\mathbf{q}}^{+}$and $\overline{\mathbf{q}}^{+}$, respectively.

The gradient of the Lagrangian can be expressed then as:

$$
\begin{aligned}
\nabla \mathcal{L}= & <\frac{\partial \mathcal{L}}{\partial \overline{\mathbf{q}}}, \delta \overline{\mathbf{q}}>+<\frac{\partial \mathcal{L}}{\partial \sigma}, \delta \sigma>+<\frac{\partial \mathcal{L}}{\partial \hat{\mathbf{q}}}, \delta \hat{\mathbf{q}}>+ \\
& +<\frac{\partial \mathcal{L}}{\partial \mathbf{q}_{f}}, \delta \mathbf{q}_{f}>+<\frac{\partial \mathcal{L}}{\partial \boldsymbol{\lambda}_{1}}, \delta \boldsymbol{\lambda}_{1}><\frac{\partial \mathcal{L}}{\partial \boldsymbol{\lambda}_{1}}, \delta \boldsymbol{\lambda}_{2}>
\end{aligned}
$$

In this analysis, we would like to retain only the fourth component of the Lagrangian, $\partial \mathcal{L} / \partial \mathbf{q}_{f}$. Cancelling the gradient of the Lagrangian with respect to the first multiplier, $\boldsymbol{\lambda}_{1}$, leads again to the original eigenvalue problem, and the cancellation with respect to the second multiplier, $\boldsymbol{\lambda}_{2}$, leads to the Navier-Stokes equations modified with the forcing term.

Playing with the third term of the Lagrangian, derivative with respect to the eigenmode, we arrive to the adjoint eigenvalue problem, confirming again that $\lambda_{1}=\hat{\mathbf{q}}^{+}$.

$$
\begin{aligned}
<\frac{\partial \mathcal{L}}{\partial \hat{\mathbf{q}}}, \delta \hat{\mathbf{q}}> & =<\boldsymbol{\lambda}_{1},(\mathbf{A}(\overline{\mathbf{q}})-\sigma) \delta \hat{\mathbf{q}}> \\
& =<(\mathbf{A}(\overline{\mathbf{q}})-\sigma)^{+} \boldsymbol{\lambda}_{1}, \delta \hat{\mathbf{q}}>=0 \\
& \mathbf{A}^{+}(\overline{\mathbf{q}}) \boldsymbol{\lambda}_{1}=\sigma^{+} \boldsymbol{\lambda}_{1} .
\end{aligned}
$$

The derivative of the Lagrangian with respect to the target eigenvalue leads to the already known normalization for the adjoint perturbation variables:

$$
\begin{aligned}
<\frac{\partial \mathcal{L}}{\partial \sigma}, \delta \sigma> & =\delta \sigma+<\boldsymbol{\lambda}_{1},-\delta \sigma \hat{\mathbf{q}}> \\
& =\delta \sigma-\delta \sigma<\boldsymbol{\lambda}_{1}, \hat{\mathbf{q}}>=0 \\
& <\hat{\mathbf{q}}^{+}, \hat{\mathbf{q}}>=1 .
\end{aligned}
$$


This time, the derivative with respect the base flow needs to be cancelled as well:

$$
\begin{aligned}
<\frac{\partial \mathcal{L}}{\partial \overline{\mathbf{q}}}, \delta \overline{\mathbf{q}}> & =<\boldsymbol{\lambda}_{1}, \frac{\partial \mathbf{A}(\overline{\mathbf{q}}) \hat{\mathbf{q}}}{\partial \overline{\mathbf{q}}} \delta \overline{\mathbf{q}}>+<\boldsymbol{\lambda}_{2}, \frac{\partial \mathbf{R}(\overline{\mathbf{q}})}{\partial \overline{\mathbf{q}}} \delta \overline{\mathbf{q}}> \\
& =<\boldsymbol{\lambda}_{1}, \mathbf{B}(\overline{\mathbf{q}}, \hat{\mathbf{q}}) \delta \overline{\mathbf{q}}>+<\boldsymbol{\lambda}_{2}, \mathbf{A}(\overline{\mathbf{q}}) \delta \overline{\mathbf{q}}> \\
& =<\mathbf{B}^{+}(\overline{\mathbf{q}}, \hat{\mathbf{q}}) \boldsymbol{\lambda}_{1}, \delta \overline{\mathbf{q}}>+<\mathbf{A}(\overline{\mathbf{q}})^{+} \boldsymbol{\lambda}_{2}, \delta \overline{\mathbf{q}}> \\
& =<\mathbf{B}^{+}(\overline{\mathbf{q}}, \hat{\mathbf{q}}) \boldsymbol{\lambda}_{1}+\mathbf{A}(\overline{\mathbf{q}})^{+} \boldsymbol{\lambda}_{2}, \delta \overline{\mathbf{q}}>=0 .
\end{aligned}
$$

Replacing $\boldsymbol{\lambda}_{1}$ with $\hat{\mathbf{q}}^{+}$and inserting Equation (2.34) into the expression above, we retrieve a linear system to obtain $\boldsymbol{\lambda}_{2}$ :

$$
\mathbf{A}(\overline{\mathbf{q}})^{+} \boldsymbol{\lambda}_{2}=-\mathbf{B}^{+}(\overline{\mathbf{q}}, \hat{\mathbf{q}}) \hat{\mathbf{q}}^{+}=-\nabla_{\overline{\mathbf{q}}} \sigma
$$

with the product of $\mathbf{B}^{+}(\overline{\mathbf{q}}, \hat{\mathbf{q}})$ and $\hat{\mathbf{q}}^{+}\left(\boldsymbol{\nabla}_{\overline{\mathbf{q}}} \sigma\right)$ as a vector, and the adjoint matrix of the Jacobian of the system already defined. The gradient of the Lagrangian with respect to a the steady force source term $\mathbf{q}_{f}$ is:

$$
<\frac{\partial \mathcal{L}}{\partial \mathbf{q}_{f}}, \delta \mathbf{q}_{f}>=<\boldsymbol{\lambda}_{2}, \delta \mathbf{q}_{f}>
$$

Hereby, assuming that the gradient of the Lagrangian and the gradient of $\sigma$ with respect to the steady force source term are equal:

$$
\Delta \sigma=<\nabla_{\mathbf{q}_{f}} \sigma, \delta \overline{\mathbf{q}}>=<\frac{\partial \mathcal{L}}{\partial \mathbf{q}_{f}}, \delta \mathbf{q}_{f}>
$$

we can write:

$$
\nabla_{\mathbf{q}_{f}} \sigma=\lambda_{2}
$$

Introducing Equation (2.46) into (2.43), the final expression for the eigenvalue sensitivity to steady forcing arises:

$$
\boldsymbol{\nabla}_{\mathbf{q}_{f}} \sigma=-\left[\mathbf{A}(\overline{\mathbf{q}})^{+}\right]^{-1} \boldsymbol{\nabla}_{\overline{\mathbf{q}}} \sigma
$$

To give physical sense to the Lagrangian multiplier $\boldsymbol{\lambda}_{2}$, we apply the rule of chain to the derivation of the eigenvalue $\sigma(\overline{\mathbf{q}}, p)$ with respect to a flow parameter, $p$

$$
\frac{\mathrm{d} \sigma}{\mathrm{d} p}=\frac{\partial \sigma^{H}}{\partial \overline{\mathbf{q}}} \frac{\partial \overline{\mathbf{q}}}{\partial p}+\frac{\partial \sigma}{\partial p} .
$$

Computing $\partial \sigma / \partial \overline{\mathbf{q}}$ and $\partial \sigma / \partial p$ may be straight-forward (at least for the first term, already calculated), but the term $\partial \overline{\mathbf{q}} / \partial p$ might not be that easy to obtain. Introducing an adjoint approach, we calculate the derivative of the residual vector $\mathbf{R}(\overline{\mathbf{q}}, p)=0$ with respect to changes in $p$, that yields:

$$
\frac{\mathrm{d} \mathbf{R}}{\mathrm{d} p}=\frac{\partial \mathbf{R}}{\partial \overline{\mathbf{q}}} \frac{\partial \overline{\mathbf{q}}}{\partial p}+\frac{\partial \mathbf{R}}{\partial p}=0
$$


from where we extract

$$
\frac{\partial \overline{\mathbf{q}}}{\partial p}=-\left(\frac{\partial \mathbf{R}}{\partial \overline{\mathbf{q}}}\right)^{-1} \frac{\partial \mathbf{R}}{\partial p} .
$$

Introducing Equation (2.50) into (2.48), yields:

$$
\frac{\mathrm{d} \sigma}{\mathrm{d} p}=-\frac{\partial \sigma^{H}}{\partial \overline{\mathbf{q}}}\left(\frac{\partial \mathbf{R}}{\partial \overline{\mathbf{q}}}\right)^{-1} \frac{\partial \mathbf{R}}{\partial p}+\frac{\partial \sigma}{\partial p}
$$

To solve this equation through an adjoint approach, we introduce the adjoint operator of the base flow by solving the equation:

$$
\left(\frac{\partial \mathbf{R}}{\partial \overline{\mathbf{q}}}\right)^{H} \overline{\mathbf{q}}^{+}=-\left(\frac{\partial \sigma}{\partial \overline{\mathbf{q}}}\right),
$$

to finally obtain:

$$
\frac{\mathrm{d} \sigma}{\mathrm{d} p}=\left(\overline{\mathbf{q}}^{+}\right)^{H} \frac{\partial \mathbf{R}}{\partial p}+\frac{\partial \sigma}{\partial p} .
$$

This last equation will be recovered on next section. We can hence correlate Equation (2.52) with Equation (2.47), and affirm that the second Lagrangian multiplier, $\boldsymbol{\lambda}_{2}$, is the adjoint operator of the base flow, $\overline{\mathbf{q}}^{+}$. 



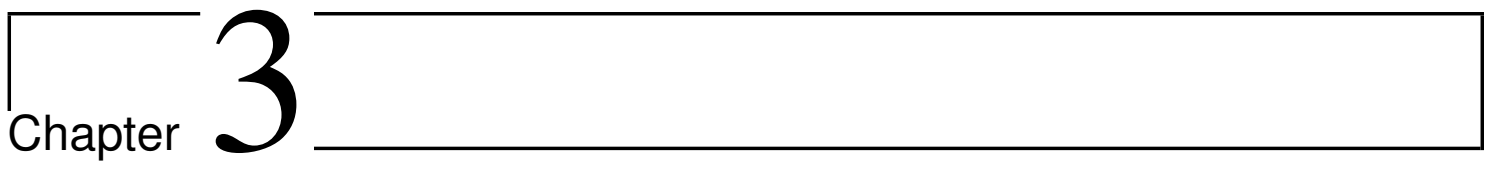

\section{Numerical Implementation}

\section{Contents}

3.1 Flow Solver - DLR-TAU Code . . . . . . . . . 27

3.1.1 Dimensionalisation of the flow conditions . . . . . . . 30

3.1.2 Construction of the Jacobian matrix . . . . . . . . . 31

3.1.3 Construction of the Hessian matrix . . . . . . . . . . 33

3.2 Stability Analysis - Large Eigenvalue Problem Solver . . 33

3.2.1 Domain reduction methodology . . . . . . . . . 35

$3.2 .2 \quad$ Explicit storage justification . . . . . . . . . . . 36

\subsection{Flow Solver - DLR-TAU Code}

All the flow calculations on this thesis have been done with the Finite Volume DLR-TAU Code[129] (TAU). The solver is a state-of-the-art commercial software, developed for the prediction of viscous and inviscid flows around complex geometries from the low subsonic to the hypersonic flow regime, employing hybrid unstructured grids. While TAU is mainly used for complex aircraft-type configurations, being one of the main CFD solvers in the aerospace industry, its extension and capabilities permit the calculation of low-speed (near the incompressible regime) and confined or wall-bounded flows.

The flow solver is a three-dimensional, parallel, multigrid code, that employs a finite volume scheme to solve the compressible flow Reynolds-averaged NavierStokes (RANS) equations. The inviscid terms are computed either with a central or with a second-order upwind scheme, whereas the viscous terms are computed with a second-order central scheme. For accelerating the convergence to steady state, which is of particular interest for the stability analysis of the flow configuration, a local time-stepping concept, a residual smoothing algorithm and a geometrical 
multigrid method are implemented. For time-accurate solutions, global and dual time-stepping schemes are implemented.

The compressible version of the RANS equations are used to model the flow, which set of equations can be written in integral form as:

$$
\frac{\partial}{\partial t} \int_{\Omega} \mathbf{q} d \Omega=-\int_{\partial \Omega} \overline{\bar{F}} \cdot \mathbf{n} d S
$$

where vector q comprises the conservative variables (density, momentum and energy) and turbulent quantities, while $\Omega$ is a control volume with boundary $\partial \Omega$ and outer normal $\mathbf{n}$. The specific heat capacities of the gas at constant volume and pressure are both assumed constant, so, consequently, it can be also defined the constant adiabatic coefficient of the ideal gas. The flux density tensor $\overline{\bar{F}}$ can be decomposed along the three Cartesian coordinate directions and comprises the inviscid, viscous (and turbulent fluxes if any) terms in the three cordinate directions $(x, y, z)$ :

$$
\begin{aligned}
& \mathbf{F}_{x}^{c}=\left[\begin{array}{c}
\rho u \\
\rho u u+p \\
\rho u v \\
\rho u w \\
\rho H u
\end{array}\right], \quad \mathbf{F}_{x}^{v}=\left[\begin{array}{c}
0 \\
\tau_{x x} \\
\tau_{x y} \\
\tau_{x z} \\
\tau_{y i} U_{i}+\phi_{x}
\end{array}\right], \\
& \mathbf{F}_{y}^{c}=\left[\begin{array}{c}
\rho v \\
\rho v u \\
\rho v v+p \\
\rho v w \\
\rho H v
\end{array}\right], \quad \mathbf{F}_{y}^{v}=\left[\begin{array}{c}
0 \\
\tau_{y x} \\
\tau_{y y} \\
\tau_{y z} \\
\tau_{y i} U_{i}+\phi_{y}
\end{array}\right], \\
& \mathbf{F}_{z}^{c}=\left[\begin{array}{c}
\rho w \\
\rho w u \\
\rho w v \\
\rho w w+p \\
\rho H w
\end{array}\right], \quad \mathbf{F}_{z}^{v}=\left[\begin{array}{c}
0 \\
\tau_{z x} \\
\tau_{z y} \\
\tau_{z z} \\
\tau_{z i} U_{i}+\phi_{z}
\end{array}\right],
\end{aligned}
$$

where $U=(u, v, w)^{T}$ is the velocity vector, $\tau$ is the viscous shear stress tensor and $\phi$ is the heat flux vector. A calorically perfect gas is assumed, defining pressure by the state equation

$$
p=(\gamma-1) \rho\left\{E-\frac{1}{2} U^{2}\right\}
$$

where $E$ is the specific total energy per unit mass and $\gamma$ is the heat capacity ratio. In addition, total enthalpy is defined as

$$
H=E+\frac{p}{\rho}
$$

The heat fluxes are given by the differential form of Fourier's law for thermal conduction,

$$
\phi=\kappa_{l} \nabla T T=\frac{p}{\rho \Re}
$$


being $\kappa_{l}$ the flow thermal conductivity. The temperature is finally recovered as a function of the pressure and density, and the universal gas constant as $T=p / \rho \Re$. The laminar viscosity, $\mu_{l}$, is calculated with the Sutherland law:

$$
\mu_{l}=\mu_{l, \infty} \cdot\left(\frac{T}{T_{\infty}}\right)^{1.5} \cdot \frac{T_{\infty}+S}{T+S},
$$

being $S$ the Sutherland reference temperature of the gas of analysis.

The addition of turbulence models is done through linear eddy-viscosity models, which contribution to the momentum equations is modelled by an increased viscosity given by the Boussinesq assumption [19, 128], being the difference between the models the way how the eddy viscosity $\mu_{t}$ is calculated. One-equation models (typically in external aerodynamic flows, Spalart-Allmaras type[136] models are used) use one extra transport equation to obtain the modified eddy viscosity, whereas on two-equation models $(k-\omega$ type[155]) two extra transport are solved for the specific kinetic turbulence energy, $k$ and dissipation rate, $\omega$.

The flow domain, $\Omega$, is spatially discretised using a dual mesh into a finite number $N$ of subdomains $\Omega_{i}(i=1, . ., N)$, where each subdomain contains $N_{f}$ faces, using a Finite Volume Methodology. Hence, tensors $\overline{\bar{F}}^{c}+\overline{\bar{F}}^{v}$ are approximated by numerical fluxes. Namely, the change of the flow conditions in a control volume is given by the normal component of the flux through the control volume $\Omega_{i}$, with normal direction $\mathbf{n}$. For example, given a hexahedral mesh element:

$$
\overline{\bar{F}} \cdot \mathbf{n}=\left\{\mathbf{F}_{x}, \mathbf{F}_{y}, \mathbf{F}_{z}\right\} \cdot\left\{\mathbf{n}_{x}, \mathbf{n}_{y}, \mathbf{n}_{z}\right\}=\mathbf{F}_{x} \mathbf{n}_{x}+\mathbf{F}_{y} \mathbf{n}_{y}+\mathbf{F}_{z} \mathbf{n}_{z} .
$$

This is schematically represented on Fig. 3.1, being equivalent for other type or deformed mesh elements using the proper direction projections.

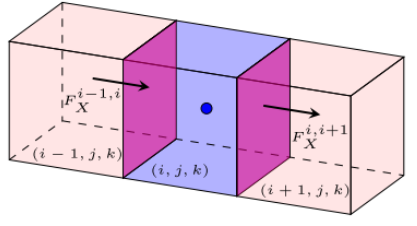

(a) X-direction fluxes

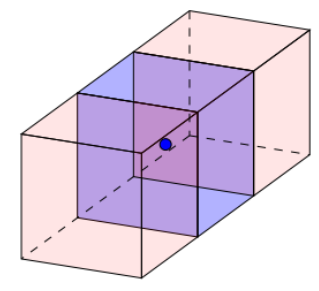

(b) Y-direction fluxes

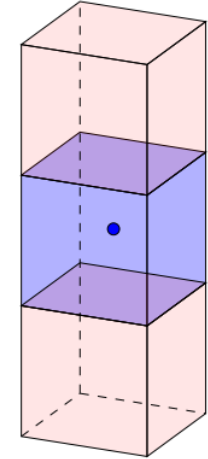

(c) Z-direction fluxes

Figure 3.1: Example of numerical fluxes balance in each direction for a threedimensional hexahedral mesh element.

Thus, following the method of lines, the spatial discretisation of system (2.1) gives rise to a system of ordinary differential equations, that can be written in general 
form for a subdomain $\Omega_{i}$ as

$$
|\Omega|_{i} \frac{\partial \mathbf{q}_{i}}{\partial t}+\mathbf{R}_{i}=\mathbf{0} \quad, \quad \mathbf{R}_{i}=\sum_{j=1}^{N_{f}} \overline{\bar{F}}_{j} \mathbf{n}_{j}, \quad i=1 \ldots N
$$

where $\mathbf{R}_{i}$ is the residual in a subdomain $\Omega_{i}$, equivalent to the flux contributions on this subdomain, and $\mathbf{q}_{i}$ represents a discretised vector state solution. Vectors $\mathbf{R}_{\mathbf{i}}$ and $\mathbf{q}_{i}$ have dimensions that depend on the number of fluid variables $N_{v}$ considered. Namely, in a two-dimensional flow, $N$ will be 4 for a laminar solution, 5 for a oneequation turbulent flow solution and 6 for a two-equation turbulent flow solution. In case of a three-dimensional flows, and additional equation would be considered for the spanwise velocity. The number of subdomains depends on the number of elements of the computational mesh.

The time-accurate three-dimensional Navier-Stokes equations are then marched in time towards steady state by a backward Euler implicit scheme, solved approximately by a LU-SGS (Lower-Upper Symmetric-Gauss-Seidel Method) iterations procedure.

\subsubsection{Dimensionalisation of the flow conditions}

Before the calculation of the flow solution starts, the initial free stream flow conditions that serve to initialize the flow variables are normally determined from the reference values, depending on the input for the inflow angle of incidence, $\alpha$, and the slip angle, $\beta$ :

$$
\begin{aligned}
\rho_{\infty} & =\rho_{\text {ref }} \\
p_{\infty} & =p_{\text {ref }} \\
u_{\infty} & =\cos (\alpha) \cdot \cos (\beta) \cdot M_{\infty} \cdot \sqrt{\gamma} \cdot \sqrt{p_{\infty} / \rho_{\infty}} \\
v_{\infty} & =\sin (\beta) \cdot M_{\infty} \cdot \sqrt{\gamma} \cdot \sqrt{p_{\infty} / \rho_{\infty}} \\
w_{\infty} & =\sin (\alpha) \cdot \cos (\beta) \cdot M_{\infty} \cdot \sqrt{\gamma} \cdot \sqrt{p_{\infty} / \rho_{\infty}}
\end{aligned}
$$

together with the ideal gas law,

$$
p_{\infty}=\rho_{\infty} R T_{\infty}
$$

and the definition of the Reynolds number, based on a characteristic length $l_{\text {ref }}$,

$$
R e=\frac{\rho_{\infty} u_{\infty} l_{c}}{\mu_{l}}
$$

The laminar viscosity, $\mu_{l}$, is calculated with the Sutherland law:

$$
\mu_{l}=\mu_{l, r e f} \cdot\left(\frac{T_{\infty}}{T_{r e f}}\right)^{1.5} \cdot \frac{T_{r e f}+S}{T_{\infty}+S},
$$


being $S$ and $T_{r e f}$ the Sutherland and the reference temperatures of the gas of analysis, with the turbulent viscosity, $\mu_{t}$ being defined by the turbulent viscosity ratio as:

$$
\mu_{t, \text { init }}=\mu_{l, \text { init }} \cdot \mu_{t, \text { ratio }} .
$$

The primitive variables (dimensional values are denoted by a bar) are later nondimensionalised for a better internal performance of the code:

$$
\begin{aligned}
\rho & =\bar{\rho} / \rho_{\infty} \\
p & =\bar{p} / p_{\infty} \\
u & =\bar{u} \cdot \sqrt{\rho_{\infty} / p_{\infty}} \\
v & =\bar{v} \cdot \sqrt{\rho_{\infty} / p_{\infty}} \\
w & =\bar{w} \cdot \sqrt{\rho_{\infty} / p_{\infty}} \\
T & =\bar{T} / T_{\infty},
\end{aligned}
$$

with the reference time, defined at the initialization of the calculation, is defined as:

$$
\bar{t}=\bar{l}_{c} / \bar{u} .
$$

This relation between the initial flow parameters and the non-dimensionalisation is of particular interest for the later calculation of the global mode frequencies. For the streamwise velocity, $u$, this relation at the moment of the flow initialization is:

$$
\begin{aligned}
u_{\infty} & =\cos (\alpha) \cdot \cos (\beta) \cdot M_{\infty} \cdot \sqrt{\gamma} \cdot \sqrt{p_{\infty} / \rho_{\infty}} \\
& =\cos (\alpha) \cdot \cos (\beta) \cdot M_{\infty} \cdot \sqrt{\gamma} \cdot \bar{u}
\end{aligned}
$$

From a physical point of view, we are interested in the time defined as

$$
t=l_{c} / u_{\infty}
$$

which when it is compared with the reference time of TAU, the following relation appears:

$$
\frac{t}{\bar{t}}=\left(\frac{\bar{u}}{u_{\infty}}\right)\left(\frac{l_{c}}{\bar{l}_{c}}\right)=\frac{1}{M_{\infty} \sqrt{\gamma}}
$$

Therefore, to recover the physical sense on the time domain for the analysis of our linear operator (the Jacobian matrix), the elements of it have to be scaled with the factor defined on (3.19)

\subsubsection{Construction of the Jacobian matrix}

One of the main advantages of the TAU solver is the presence of an analytic implementation of the Jacobian matrix, $\partial \mathbf{R} / \partial \mathbf{q}$, which serves both the implicit version of the flow solver and the Linear Frequency Domain solver (which is not contemplated 


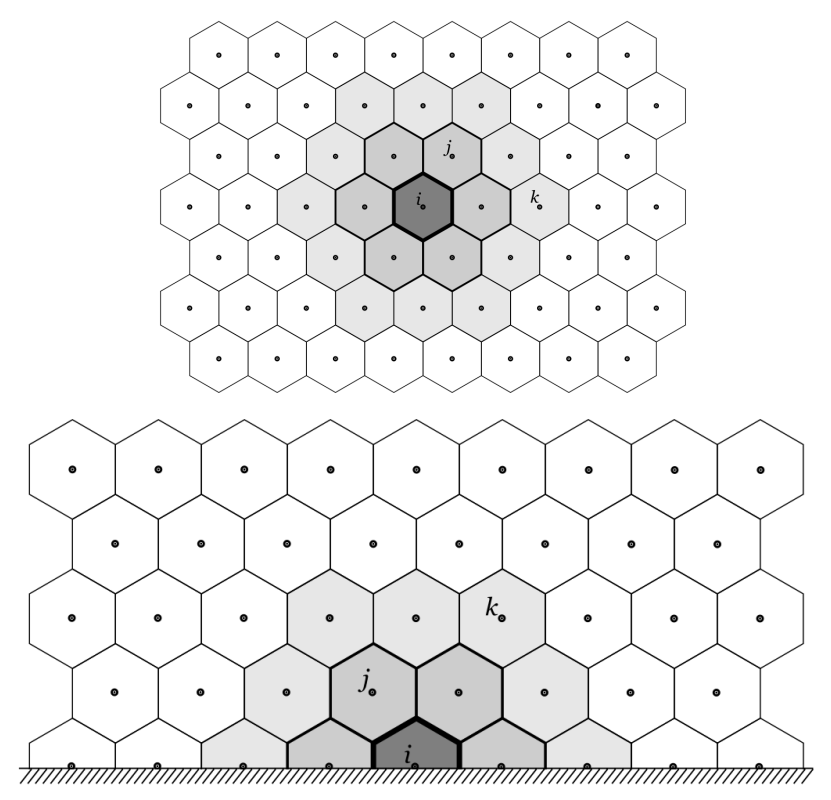

Figure 3.2: Compact second-order stencil. Grey cells correspond to the immediate neighbours, and light grey cells are the immediate next-neighbours. Images modified from [35].

on this thesis). The linear version of the Jacobian relies on the first-discretise-thenlinearise philosophy, and includes the implementation of many boundary conditions and turbulence models of one and two equations. Details of the calculation and implementation of the analytic Jacobian matrix can be consulted in the work of Dwight[35], so only a brief description is given here.

The Residual and the state vector, $\mathbf{R}$ and $\mathbf{q}$, are vectors of size $N \times N_{v}$, where $N$ is the number of nodes in the grid and $N_{v}$ corresponds to the number of equations per node. Then, $\partial \mathbf{R} / \partial \mathbf{q}$ is a matrix with dimensions $\left(N \times N_{v}\right) \times\left(N \times N_{v}\right)$, with its structure dependent on the ordering of the degrees of freedom. If the ordering of the vectors is considered in the form

$$
\left(\rho_{0}, u_{0}, v_{0}, p_{0}, \rho_{1}, u_{1}, \cdots, \rho_{n-1}, u_{n-1}, v_{n-1}, p_{n-1}\right),
$$

we can consider the Jacobian as an $N \times N_{v}$ matrix of $N \times N_{v}$ blocks. With this assumption, each block matrix can be expressed as $\partial \mathbf{R}_{i} / \partial \mathbf{q}_{j}$, obtained by differentiating the components of $\mathbf{R}_{i}$ with respect to the $N_{v}$ components of $\mathbf{q}_{j}$. However, $\mathbf{R}_{i}$ is a function of only a small number of $\mathbf{q}_{j}$ in the vicinity of node $i$, namely its first-order or second-order neighbours. An example of the affected discretised nodes is shown on Figure 3.2. If a point $j$ is not in the stencil of $\mathbf{R}_{j}$, its corresponding entry on the Jacobian will be zero.

For a better handling of the Jacobian, the TAU code was modified to store the full sparse matrix in memory using a Compressed Sparse Row (CSR) distribution, written in NetCDF format[148]. 


\subsubsection{Construction of the Hessian matrix}

Since the TAU solver only provides the Jacobian matrix, we consider the following procedure to compute the second derivatives for the Hessian matrices, required to compute Equation (2.34). From its definition, it follows that $\mathbf{B}(\overline{\mathbf{q}}, \hat{\mathbf{q}})$ is a linear function of $\hat{\mathbf{q}}=\hat{\mathbf{q}}_{R e}+i \hat{\mathbf{q}}_{I m}$ and a non-linear function of $\overline{\mathbf{q}}$ through the Jacobian of $\mathbf{R}(\overline{\mathbf{q}})$. Thus, it can be decomposed into its real and imaginary parts:

$$
\mathbf{B}(\overline{\mathbf{q}}, \hat{\mathbf{q}})=\mathbf{B}(\overline{\mathbf{q}}, \hat{\mathbf{q}})_{R e}+i \mathbf{B}(\overline{\mathbf{q}}, \hat{\mathbf{q}})_{I m}=\frac{\partial \mathbf{A}(\overline{\mathbf{q}}) \hat{\mathbf{q}}_{R e}}{\partial \overline{\mathbf{q}}}+i \frac{\partial \mathbf{A}(\overline{\mathbf{q}}) \hat{\mathbf{q}}_{I m}}{\partial \overline{\mathbf{q}}} .
$$

Additionally, one can define (for either the real or imaginary part of $\hat{\mathbf{q}}$ ) the directional derivative of $\mathbf{A}(\overline{\mathbf{q}})$ along $\hat{\mathbf{q}}$ as:

$$
\left.\frac{\partial \mathbf{A}(\overline{\mathbf{q}}) \hat{\mathbf{q}}}{\partial \mathbf{q}}\right|_{\mathbf{q}=\overline{\mathbf{q}}}=\frac{\mathbf{A}\left(\overline{\mathbf{q}}+\epsilon_{1} \hat{\mathbf{q}}\right)-\mathbf{A}(\overline{\mathbf{q}})}{\epsilon_{1}}+\mathcal{O}\left(\epsilon_{1}^{2}\right) .
$$

Therefore, neglecting order $\mathcal{O}\left(\epsilon_{1}^{2}\right)$ terms, one can apply this definition to the calculation of the sensitivity matrix:

$$
\mathbf{B}(\overline{\mathbf{q}}, \hat{\mathbf{q}})=\frac{\partial \mathbf{A}(\overline{\mathbf{q}}) \hat{\mathbf{q}}}{\partial \overline{\mathbf{q}}}=\frac{1}{\epsilon_{1}}\left\{\left[\mathbf{A}\left(\overline{\mathbf{q}}+\epsilon_{1} \hat{\mathbf{q}}_{R e}\right)-\mathbf{A}(\overline{\mathbf{q}})\right]+i\left[\mathbf{A}\left(\overline{\mathbf{q}}+\epsilon_{1} \hat{\mathbf{q}}_{I m}\right)-\mathbf{A}(\overline{\mathbf{q}})\right]\right\} .
$$

For a detailed analysis on the influence of $\epsilon_{1}$, the reader is referred to [20].

Having computed the Jacobian and Hessian matrices, it is possible to obtain the sensitivity to base flow and external forcing following equations (2.34) and (2.47), as described in Section 2.2.

\subsection{Stability Analysis - Large Eigenvalue Problem Solver}

The stability behaviour of a hydrodynamic system can be obtained through the analysis of a linearised operator of the non-linear flow solution. The eigenvalues of the operator, namely the Jacobian matrix of the flow solution, describe the asymptotic behaviour of the flow system. For each eigenvalue there is an associate eigenvector that represents the underlying physical feature (density, momentum in the spatial directions $(x, y, z)$, energy and turbulent viscosity), which will eventually decay or grow depending on the sign of the real part of the eigenvalue. The resources for solving those large eigenvalue problems have quickly evolved in last decades, with an increasing number of efficient methodologies an numerical methods to tackle the problem[113, 114, 116, 157]. Of particular interest are the iterative methods based on a Krylov subspace projection[112], where only a small portion $N_{e v}$ of the total number of eigenvalues of the matrix are obtained. These methods normally involve 
the solution of $N_{e v}$ linear systems, a number at least equal to the size of the desired Krylov subspace, that in turn could be solved directly (computing the inverse of the operator, i.e. by means of a Lower-Upper decomposition[57]) or iteratively (using a preconditioned subspace approximation, i.e. GMRES[115].

Krylov subspace methods are based on the projection of the square matrix A $(n \times n)$ into a subspace of lower dimension, normally based on a Rayleigh-Ritz projection:

$$
\mathbf{H}=\mathbf{V}^{T} \mathbf{A V},
$$

with $\mathbf{V}(n \times m$, with $m<<n)$ containing the vectors of an orthonormal basis of a subspace, with $\mathbf{V}^{T} \mathbf{V}=\mathbf{I}$. $\mathbf{H}$ is a square matrix $(m \times m)$, projection of $\mathbf{A}$ into the subspace $\mathbf{V}$, whose eigenvalues of system

$$
\mathbf{H p}=\alpha \mathbf{p}
$$

approximate a portion of the total spectra of $\mathbf{A}$. The eigenpair $\left(\alpha_{i}, \mathbf{p}_{i}\right)$ correlate with the approximation $\left(\tilde{\sigma}_{i}, \tilde{\mathbf{q}}_{i}\right)$ of the eigenpair of the original matrix $\left(\sigma_{i}, \mathbf{q}_{i}\right)$ as

$$
\begin{aligned}
\tilde{\sigma}_{i} & =\alpha_{i}, \\
\tilde{\mathbf{q}}_{i} & =\mathbf{V} \mathbf{p}_{i} .
\end{aligned}
$$

$\tilde{\sigma}_{i}$ and $\tilde{\mathbf{q}}_{i}$ are the Ritz values and Ritz vectors, respectively. Starting from an initial (usually random) vector, $\mathbf{q}_{1}$, the Arnoldi algorithm builds the Krylov subspace by means of repetitive matrix-vector product in the form of a power method (q, Aq, $\left.\mathbf{A}^{2} \mathbf{q}, \ldots, \mathbf{A}^{m-1} \mathbf{q}\right)$. At each iteration the new vector is orthonormalised, resulting in a final Hessenberg matrix $\mathbf{H}$. However, the $m$ eigenvalues of the matrix $\mathbf{H}$ obtained through the Arnoldi algorithm converge to the $m$ largest eigenvalues of $\mathbf{A}$. To capture the region of the complex plane where the eigenvalues of interest lay (normally the least stable, or "rightmost"), a shift-and-invert spectral transformation[114] is used. It converts the eigenvalue problem $\mathbf{A q}=\sigma \mathbf{q}$ into

$$
(\mathbf{A}-\nu \mathbf{I})^{-1} \mathbf{q}=\theta \mathbf{q} .
$$

This transformation is used to find the eigenvalues near $\nu$ (complex shift parameter, pointing the are of interest of the complex plane), since the eigenvalues of the modified operator, $\theta$, with largest magnitude, correspond to the eigenvalues of the original problem, $\sigma$, that are closest to the shift $\nu$ value. The eigenvectors remain unchanged, and the eigenvalues are recovered by rescaling them as $\sigma=\nu+1 / \theta$.

However, this spectral transformation requires the inversion of the matrix $\mathbf{A}$ at each iteration on the generation of the Krylov subspace, now fed with the matrix vector product $(\mathbf{A}-\lambda \mathbf{I})^{-1} \mathbf{q}$, which makes this procedure computationally expensive. Moreover, due to the stiffness associated to the underlying compressible NavierStokes equations and turbulence models, matrix $\mathbf{A}$ is badly conditioned, so iterative methods to solve the linear system fail to reach a proper convergence. In this work, a full Lower-Upper (LU) factorization is performed for the Jacobian matrix 
A. This strategy, used here in a compressible finite volume context, has also been followed in stability analysis of incompressible flows in finite elements discretisations and in the context of spectral methods [20, 29, 40] and highly compressible flows with turbulence modelling[61]. Unfortunately, the full LU decomposition scales as the cube of the number of unknowns $\left(\left(N_{v} \times N\right)^{3}\right)$, becoming the bottleneck of the overall process and consuming a large amount of computational resources[61]. To partially alleviate this requirement, sparse matrix format and parallel algorithms are used. This is done through the PETSc package[8, 9, 10], combining an OpenMP and MPI implementation of the algorithms. The MUMPS library[5] is used for the LU decomposition, combined with the Implicitly Restarted Arnoldi Method (IRAM) algorithm implemented in the ARPACK library[72] to obtain the eigenvalues of $\mathbf{A}$ and $\mathbf{A}^{+}$

\subsubsection{Domain reduction methodology}

As seen on Section 3.1.2, the use of a discrete approach on the calculation of the Jacobian matrix implies that the information contained at each grid node affects only to itself and to its first and second neighbours in each spatial direction. A proper management of the Jacobian data permits to exploit this distribution to, for example, avoid the interference of certain boundary conditions, or to analyse the stability behaviour of only a certain region of the flow. This is of particular interest to reduce the size of the large operator used on the eigenvalue problem, as those generated on high Reynolds number simulations or 3D flows calculations.

If the relevant information of the Jacobian matrix is extracted, the large eigenvalue solver has to be solved for only a reduced portion of the domain used on the calculation of the flow solution. For completeness of exposition, we shortly reproduce here the mathematical foundation of this method, additional information can be found in the work of Sanvido[120].

Arnoldi method belongs to the broad category of projection methods which aim to obtain the part of the spectrum of matrix $\mathbf{A}$ where the dominant eigenpairs lay. The domain reduction (DR) strategy can be considered, somehow, a projection technique where the original matrix is reduced to a computationally affordable one. The projection in the domain reduction procedure is a purely geometrical technique; starting from a domain of reference $\Omega_{n}$, defined from the computational mesh, and a vector of unknowns $\mathbf{q}_{\mathbf{n}}$, comprising the variables involved in the Linear NavierStokes equations, we consider a subdomain of the computational mesh $\Omega_{m}$, a region of interest, which contains $m<n$ degrees of freedom. For simplicity, we order the unknowns related to the subdomain in the first positions, in the global vector of unknowns qu. Namely,

$$
\mathbf{q}_{\mathbf{n}}=\left\{q_{1} \cdots q_{n}\right\}^{T}=\left\{q_{\{1 \cdots m\}} \mid q_{\{(m+1) \cdots n\}}\right\}^{T}
$$


under this assumption, the projection of the unknowns from $\Omega_{n}$ to $\Omega_{m}$ is easily performed through the projection operator, $\mathbf{P}$ defined as:

$$
\mathbf{q}_{\mathbf{m}}=\mathbf{P q}_{\mathbf{n}} \text { with } \mathbf{P}=\left(\begin{array}{ccccc}
1 & 0 & 0 & \cdots & 0 \\
0 & 1 & 0 & \cdots & 0 \\
0 & \vdots & \vdots & \ddots & 1 \\
0 & 0 & \cdots & 0 & 0 \\
0 & 0 & \cdots & 0 & 0
\end{array}\right)_{(n, m)}
$$

where the first $m$ rows of $\mathbf{P}$ form an identity matrix of order $m$ and the remaining $(n-m)$ rows are identically zero. It is easily checked that $\mathbf{P}$ is a orthonormal projector and its columns form a orthonormal basis on the subdomain defined by $\Omega_{m}$, so the projection of the Jacobian $\mathbf{A}_{\mathbf{n}}$ onto this subspace is easily computed as:

$$
\mathbf{A}_{\mathbf{m}}=\mathbf{P}^{\mathbf{T}} \mathbf{A}_{\mathbf{n}} \mathbf{P}
$$

where the superscript $T$ stands for the transpose and $\mathbf{A}_{\mathbf{m}}$ the orthonormal projection of $\mathbf{A}_{\mathbf{n}}$ in the subspace of dimension $m$.

Hence, the original Jacobian (in $\Omega_{n}$ ) can be replaced by its projection (in $\Omega_{m}$ ) and the "reduced" eigenpairs computed. Sanvido et al.[119] showed that DR technique permits to recover the most relevant disturbances related to the specific region of interest, with the added value of filtering part of the spectra that is not relevant for that particular region. The authors proved that the approximation will be valid as long as the reduced domain contains the structural sensitivity region of the dominant eigenmodes, fact that was also suggested by Giannetti and Luchini[46].

\subsubsection{Explicit storage justification}

One of the main discussions nowadays, is related with the way the Jacobian matrix should be managed. The dimension of the matrix grows with both the number of mesh elements and the number of variables present in the flow solution $\left(N \times N_{v}\right)$. Hence, the analysis of complex geometries (even for two-dimensional flows) or with the presence of turbulence variables will rapidly increase the size of the linear operator. This issue has led to the apparition of the so-called "Matrix-Free" methodologies, where the Jacobian matrix is never explicitly stored. The matrix is only managed to evaluate a matrix-vector product that can be used on a Krylov-Schur method to recover the least-damped direct and adjoint global modes[41]. However, the evaluation of the eigenvalue sensitivities require some matrix manipulation (including the calculation of the Hessian matrix of the flow solution), that can result in large computational costs. On the contrary, the use of an explicit storage of the matrix makes these evaluations easier and more efficient, with an adjoint operator that is obtained by just transposing the matrix.

All the cases of study considered in this Thesis are two-dimensional, with the computational costs for the stability analysis managed by the DR technique (Sec. 
3.2.1), and our main interest is on the evaluation of the adjoint operators and the extraction of eigenvalue sensitivities. Therefore, an explicit storage of the Jacobian matrix was chosen to perform the analyses here gathered. 



\section{Part II}

\section{Validation}



$\Gamma_{\text {Chapter }}$

\section{Numerical Validation}

\section{Contents}

4.1 Laminar Flows . . . . . . . . . . . . . . . 42

4.1 .1 Cylinder wake first instability . . . . . . . . . . . 42

4.1.1.1 Introduction . . . . . . . . . . . . . . 42

4.1.1.2 Results ................. . 42

4.1.2 Channel sudden expansion instability . . . . . . . . 45

4.1.2.1 Introduction . . . . . . . . . . . . . . 45

4.1 .2 Results . . . . . . . . . . . . 47

4.2 Turbulent Flows . . . . . . . . . . . . 49

4.2 .1 D-Cylinder turbulent wake . . . . . . . . . . . . 49

4.2.1.1 Introduction . . . . . . . . . . . . 49

4.2 .1 .2 Results . . . . . . . . . . . . 50

In this Chapter the numerical methods and theoretical approach proposed on Chapters 2 and 3 are tested against laminar and turbulent flow benchmark cases. The stability analysis of laminar flows is evaluated by revisiting the wake first instability of the cylinder flow and the channel sudden expansion flow. These are two examples of low-speed flows analysis, that also serve to evaluate the methodology for both open and wall-bounded flows. The turbulent regime is explored by a shear flow, the D-cylinder wake instability. For the laminar cases a steady solution of the flow is obtained, together with the eigenvalues and eigenvectors of the linearised system, recovering the hydrodynamic global modes responsible of the instability. For the turbulent case, a base flow approach is no longer accurate, so a mean flow approach is employed. In addition, the sensitivity of the least stable eigenvalue to base flow modifications and steady forcing are recovered, together with the eigenvalue structural sensitivity. 


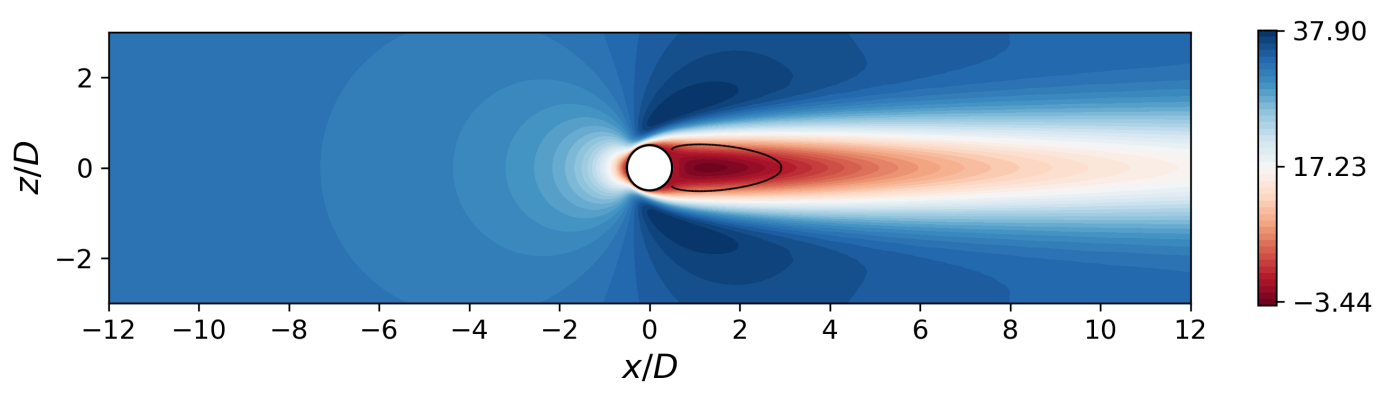

Figure 4.1: Streamwise velocity contours of the symmetric base flow solution, at $R e=45$. The streamline of the recirculation area is depicted with a black line. Only part of the computational domain is shown in the image.

\subsection{Laminar Flows}

\subsubsection{Cylinder wake first instability}

\subsubsection{Introduction}

The steady wake flow existing behind a cylinder body immersed in a laminar flow presents a symmetry with respect to its mid plane until the Reynolds number is increased beyond a critical value, $R e_{c} \approx 47[106]$. The symmetry breaking occurs via a Hopf bifurcation [97, 137], forming the characteristic Von-Karman street behind the body. This is probably one of the most studied flow cases in hydrodynamic stability, and it works as perfect example of bluff body wakes. For a complete description of the cylinder wake dynamics, the reader is referred to the work of Williamson et al.[156]. We reproduce in what follows the results obtained by Mettot et al.[89] and Browne et al.[20] on the calculation of the sensitivity fields for the eigenvalue related with the cylinder wake global instability using a discrete framework.

A laminar viscous flow around an infinite circular cylinder is considered, with the Reynolds number is fixed to $R e=45$, and a Mach number of $M=0.2$. The flow solution is time-marched towards a two-dimensional steady state, ensuring flow convergence with density residuals values lower than $10^{-8}$. To ensure a symmetric wake with respect to the XY plane, only the upper half of the cylinder geometry and the control volume are simulated, imposing a symmetry boundary condition on the mid plane. The flow solution and mesh nodes are later mirrored to obtain the linear Jacobian matrix from the complete configuration (Fig. 4.1.)

\subsubsection{Results}

The eigenvalue problem is solved using a direct method based on a LU decomposition and a Shift-and-Invert spectral transformation, with a shift value close to the expected imaginary value of the eigenvalue (based on the Strouhal number obtained on the non-steady simulations). A pair of complex eigenvalues associated with the 
Eigenvalue reduced frequency, $\sigma_{i} / 2 \pi$

\begin{tabular}{ll}
\hline \hline Crouch et al.[25], $R e=50$ & 0.117 \\
Giannetti and Luchini[46], $R e=45$ & 0.117 \\
Marquet et al.[77], $R e=46.8$ & 0.116 \\
Present study, $R e=45$ & 0.115
\end{tabular}

Table 4.1: Eigenvalue associated frequency literature revision.
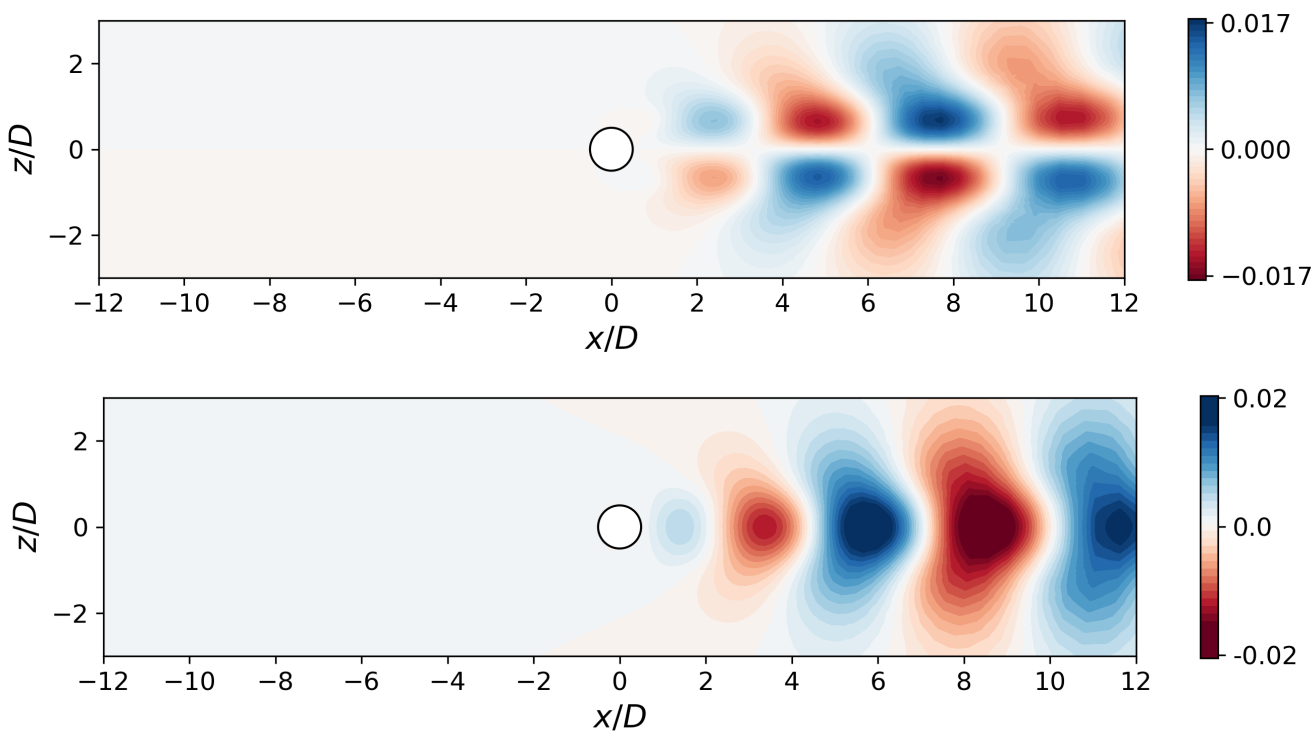

Figure 4.2: Leading global mode at $R e=45$. Real part of the streamwise velocity (upper image) and vertical velocity (lower image) perturbations.

global mode responsible of the symmetry breaking of the cylinder wake are recovered. The imaginary value of the eigenvalue is compared with different references in Table 4.1.

The shape of the global mode is defined by the antisymmetric distribution of density, energy and streamwise velocity perturbations, with the vertical velocity perturbations having alternating direction as they develop downstream the cylinder body. The adjoint global modes have a stronger intensity around the detachment areas of the cylinder, highlighting the receptivity to momentum forcing of those regions. Their spatial structures extend upstream, as they represent the influence of the incoming flow conditions. Both, direct and global modes, are shown in Figures 4.2 and 4.3

The overlapping of the two global modes, direct and adjoint, recovers the structural sensitivity field. A comparison with the results of Ref.[46] is shown in Figure 4.4. The eigenvalue sensitivity to base flow modifications and steady forcing are easily computed using Equations 2.34 and 2.47, through the Hessian and adjoint operators of the Jacobian matrix. A complex field is recovered, with sensitivities 

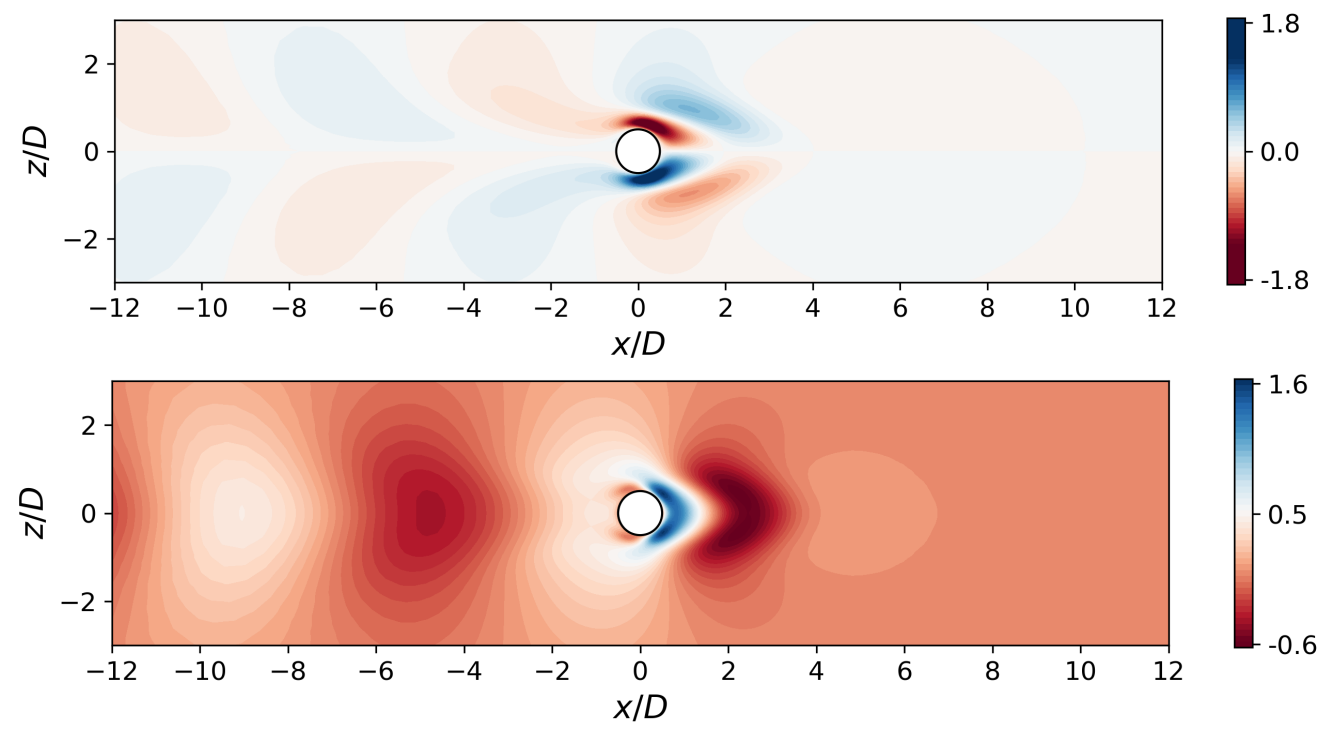

Figure 4.3: Leading adjoint global mode at $R e=45$. Real part of the streamwise velocity (upper image) and vertical velocity (lower image) perturbations.
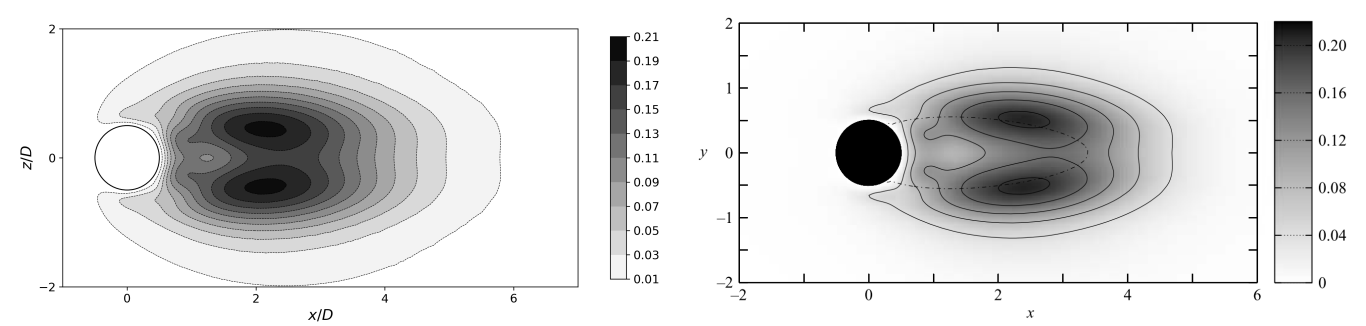

Figure 4.4: Structural sensitivity of the cylinder wake first instability. Left: Results of the present study. Right: Results from Giannetti and Luchini[46], reprinted under the permission of Cambridge University Press.

for the real (amplification rate) and imaginary part (pulsation) of the eigenvalue, named respectively as $\nabla_{\overline{\mathbf{q}}} \sigma_{r}$ and $\boldsymbol{\nabla}_{\overline{\mathbf{q}}} \sigma_{i}$. As a vector field, both the modulus and the direction of the gradients (both based on its momentum components) can be extracted to evaluate the sensitivity regions. It was observed on the works of Browne et al.[20] and Mettot et al.[88] that the eigenvalue sensitivity with respect to base flow modifications differs from the results from Marquet et al.[77]. Mettot et al. argument that the differences were due to the compressibility effects of their approach, as the results of Marquet et al. used incompressible equations on their formulation. These differences are not surprising, as a comparison of the two physical gradients based on base flow dynamic changes would have no sense due to the different dynamics used on the two approaches (incompressible vs compressible). However, the tendencies observed in the sensitivities are similar, with the orientation of the gradients having similar orientation inside the recirculation bubble. 

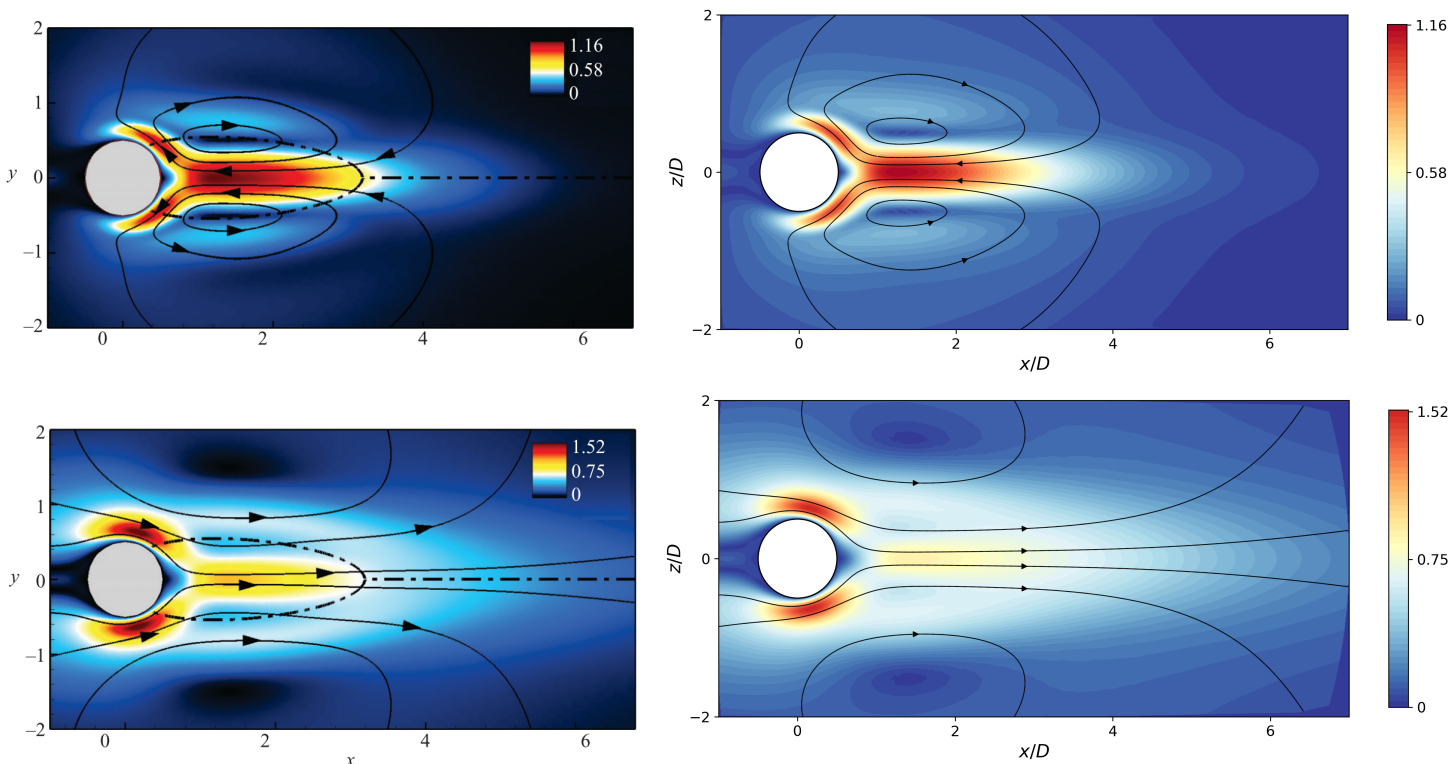

Figure 4.5: Sensitivity to the application of a steady forcing of the least stable eigenvalue at $R e=45$. Results of present study of the growth rate sensitivity $\nabla_{\overline{\mathbf{q}}} \sigma_{r}$ (upper right) and for the associated frequency $\nabla_{\overline{\mathbf{q}}} \sigma_{i}$ (lower right), and reprinted from Marquet et al.[77](upper and lower left) with the permission of Cambridge University Press.

The sensitivity of the eigenvalue with respect to the application of steady forcing is obtained solving the linear system (2.47) using the adjoint operator $\mathbf{A}^{+}$. In this case the perturbation takes into account the inherent physics of the problem[88], and the results for different formulations can be compared. The gradients are plotted in Figure 4.5, and compared with the results of Marquet et al.[77], showing excellent agreement. The depicted streamlines indicate the direction in which a steady forcing would have a positive effect on the respective gradient. Hence, a perturbation in the opposite direction to the flow inside the recirculation bubble or near the detachment points will increase the amplification rate of the eigenvalue proportionally to the local value of the sensitivity gradient $\boldsymbol{\nabla}_{\mathbf{q}_{\mathbf{f}}} \sigma_{r}$. In the same way, the same perturbation will reduce the associated frequency of the eigenvalue proportionally to the local value of $\boldsymbol{\nabla}_{\mathbf{q}_{\mathbf{f}}} \sigma_{i}$. These effects are further explored in Chapter 6 .

\subsubsection{Channel sudden expansion instability}

\subsubsection{Introduction}

The two-dimensional channel flow in a sudden expansion (a schematic representation is shown in Figure 4.6) is characterised by the apparition of non-symmetrical flow configurations after a critical Reynolds number[1, 22]. This bifurcation occurs via a Pitchfork bifurcation[4, 13, 52], without any time-dependent variations 


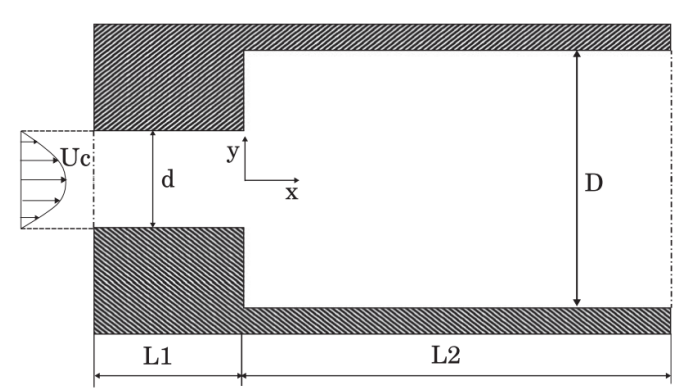

Figure 4.6: Channel sudden expansion geometry.

in two-dimensional configurations[38]. Experimental analysis suggested that the bifurcation was also influenced by the expansion ratio $(E R=d / D)$ and the aspect ratio $\left(d / L_{z}\right.$, with $L_{z}$ as the spanwise dimension) of the the channel[4, 33, 38]. On flow configurations with $R e<R e_{c}$ (being $R e_{c}$ the critical Reynolds number for a particular $E R$ ), the flow presents two symmetric recirculation regions at the corners of the expansion, its size influenced by the $E R$ and the Reynolds number. On the tipping point, $R e \approx R e_{c}$, one of the recirculation regions starts to grow at the expense of the other and a lift-up mechanism ends up deflecting the flow towards the side of the smaller recirculation area in a Coanda effect style. The stability analysis of a two-dimensional channel sudden expansion was carried out by most of the mentioned references, showing a single eigenvalue that becomes unstable at the critical Reynolds number. For higher expansion ratios, the flow becomes unstable at lower Reynolds numbers, recovering the free jet flow for $D>>1$ [4]. The transition to an oscillatory behaviour occurs via three-dimensional mechanisms[30, 38, 49], and cannot be replicated on a two-dimensional numerical or experimental analysis. The symmetry break prolongs to the turbulent regime, where the critical Reynolds number is no longer the critical parameter but the expansion ratio of the expansion, with a critical value of $E R=1.5[1,135]$.

On a laminar regime, Fani et al.[37] obtained the sensitivity gradients using the mathematical approach developed by Marquet et al.[77]. In a continuous and incompressible framework, they obtained the eigenvalue sensitivity fields for an unstable regime, completing the analysis with the extraction of the optimal regions to passive control, that were validated via DNS computation on the same publication. As a validating example of wall-bounded flows and steady global instabilities, their results are here reproduced.

We consider the two-dimensional channel expansion depicted in Figure 4.6 with a expansion ratio $E R=3$. A low speed regime $(M=0.05)$ is set for the incoming flow conditions, leading to the utilization of a preconditioner ${ }^{1}$ to avoid loosing accuracy with the compressible solver TAU. For a $E R=3$ the flow becomes unstable (steady, non-symmetric) for a Reynolds number $R e_{c} \approx 81$. For the sake

\footnotetext{
${ }^{1}$ Details of the implementation and accuracy of the TAU preconditioning can be consulted on Melber et al.[83].
} 

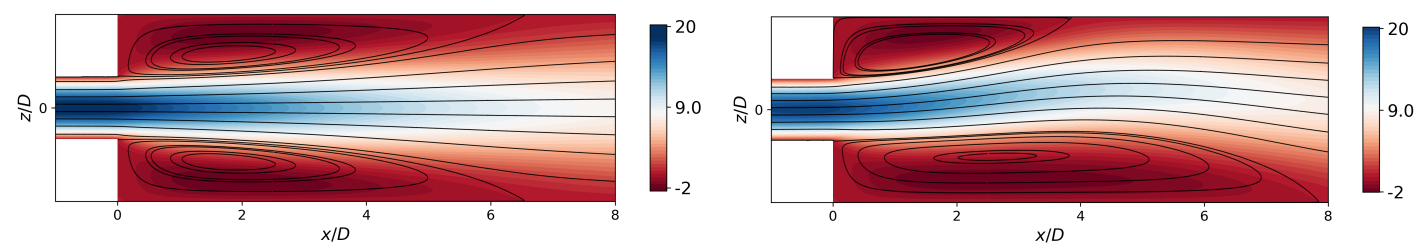

Figure 4.7: Streamwise velocity contours for the symmetric (left) and non-symmetric (right) flow configurations, at $R e=90$.

of validation, we replicate the results of Fani et al.[37] for a Reynolds number of $R e=90$. To ensure a symmetric flow with respect to the mid-plane, only half of the domain is considered for the flow simulation, and mirrored into the XY plane after the solution is converged. For completeness, an additional simulation with a non-symmetric flow configuration is also obtained. For both half and full domains simulations, a fully developed channel flow obtained from a previous two dimensional straight channel simulation was imposed at the inlet, setting up the Reynolds number as $R e=U_{c} d / \nu$ with $U_{c}$ being the maximum velocity of the incoming flow.

\subsubsection{Results}

Contours of the flow solutions are shown in Figure 4.7. The lengths of the recirculation bubbles for $R e=90$, measured by the farthest coordinate of the zero velocity streamlines of the bubbles, are gathered in Table 4.2. DLR-TAU seems to overpredict the length of the main recirculating bubble compared to the results in the literature, with a difference of approximately $3 \%$, that is considered valid for the sake of the validation of the methodology.

The stability analysis of the symmetric configuration allows to recover the global modes of the unstable (rightmost) eigenvalue. To avoid the interference of the inlet/outlet boundary conditions on the stability analysis, the domain reduction methodology (see Section 3.2.1) is used to crop the domain of the stability analysis. This technique permits not only to reduce the size of the analysed matrix, but to exploit the discrete approach formulation of the Jacobian matrix, as the omission of the boundary nodes and their second-order stencil neighbours eliminates the presence of the contribution of these nodes without creating artificial boundary conditions.

\begin{tabular}{lcc} 
& Symmetric flow $\left(x_{r}\right)$ & Asymmetric flow $\left(x_{r_{u p}} / x_{r_{l o w}}\right)$ \\
\hline \hline Fani et al.[37], $R e \approx 90$ & 6.308 & 7.584 \\
Alleborn et al.[4], $R e \approx 85$ & 6.024 & 7.315 \\
Battaglia et al.[14], $R e \approx 90$ & {$[-]$} & 7.047 \\
Present study, $R e=90$ & 6.547 & 7.839
\end{tabular}

Table 4.2: Recirculation bubble length literature revision. Units nondimensionalised with the inlet height. 

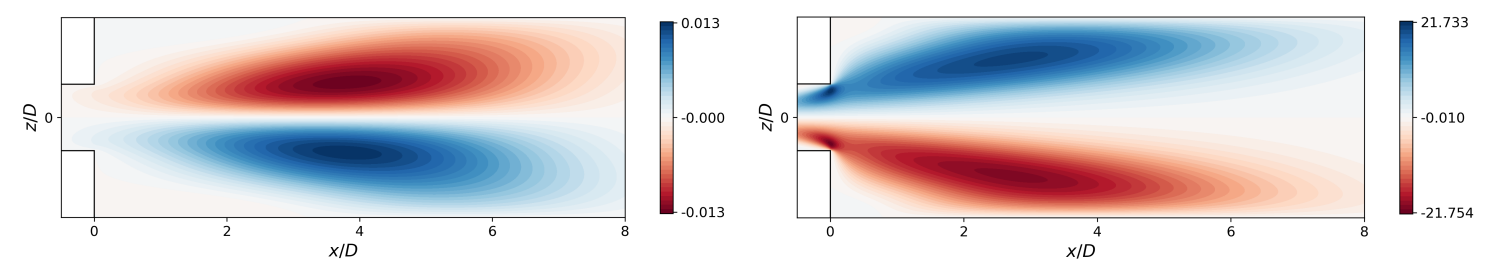

Figure 4.8: Direct (left) and adjoint(right) global modes of the sudden expansion global instability.
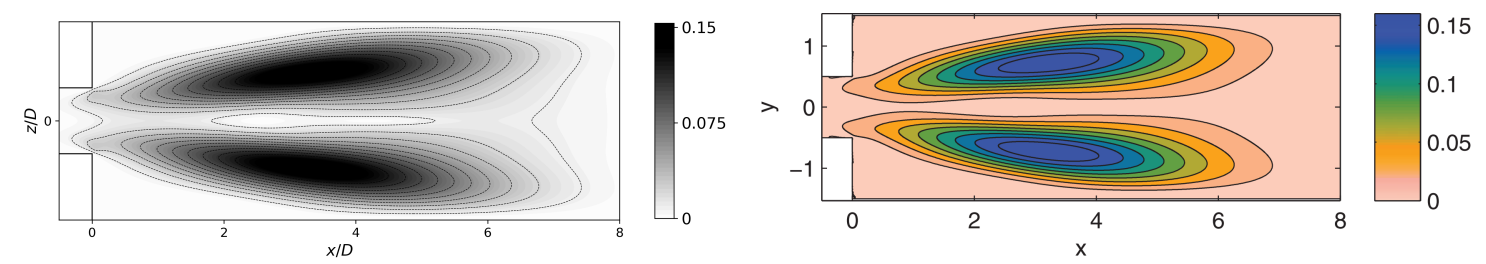

Figure 4.9: Sensitivity of the eigenvalue with respect to a structural perturbation. Left: current analysis. Right: results from Ref.[37], Reprinted from Fani et al.[37], with the permission of AIP Publishing.
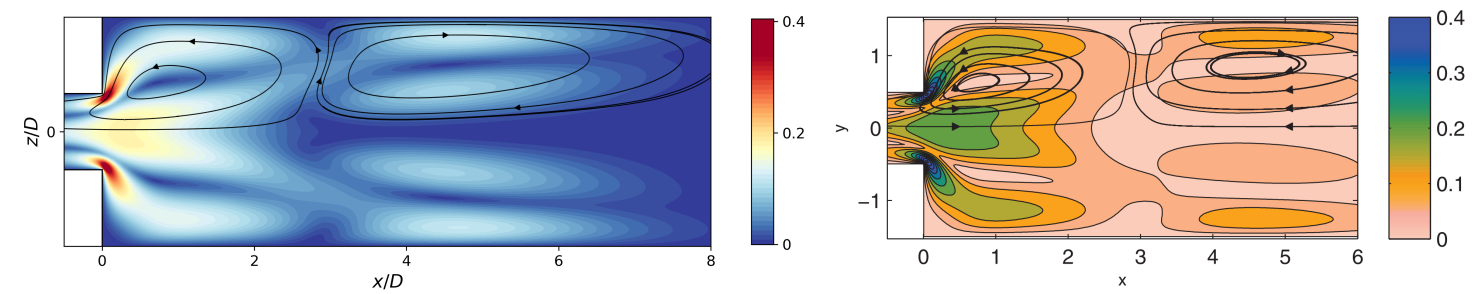

Figure 4.10: Sensitivity of the eigenvalue with respect to the application of a steady forcing. Left: current analysis. Right: results from Ref.[37], Reprinted from Fani et al.[37], with the permission of AIP Publishing.

Global and adjoint modes momentum contours are shown in Figure 4.8, where the anti-symmetric spatial distribution of the perturbations is visible. The adjoint mode presents maximum values at the corners of the expansion and within the recirculation regions. The overlapping of both, direct and adjoint global modes, results on the structural sensitivity field, from where the location of the core of the instability is recovered (Fig.4.9). The sensitivity to base flow modifications and steady forcing are calculated applying Equations (2.34) and (2.47). Note that, due to the null imaginary part of the unstable eigenvalue, only the real part of the sensitivity gradients are non-zero. A comparison with the results of Fani et al.[37] is shown in Figure 4.10. The resultant spatial distribution of the gradients is symmetric, with maximum local value around the expansion corners and decaying downstream. 


\subsection{Turbulent Flows}

\subsubsection{D-Cylinder turbulent wake}

\subsubsection{Introduction}

This validation case serves to present an introduction to the analysis of mean flows, were the stability analysis of a steady state of the flow is no longer valid. Far from the bifurcation point, we are no longer interested in the growth rate of the eigenvalue (already unstable), but on the associated frequency of the leading global mode. Due to an increment in the flow oscillations frequency as the oscillations amplitude grow, there is an underprediction of the associated Strouhal number of the flow phenomenon obtained via a base flow approximation[11]. In fully developed flows, that comply with limit cycle conditions mentioned in Section 2.1.2, a mean flow analysis is hence recommended.

An example of the application of the methodology considered in this thesis to turbulent flows is given here by the analysis of the D-shaped cylinder, immersed in a turbulent crossed flow at a moderate Reynolds number. This sensitivity of the wake on a D-shaped cylinder was first experimentally investigated by Parezanovic and Cadot[101], and numerically correlated by Meliga et al.[86] and Mettot et al.[88, 90]. Meliga et al. considered a 2D mean flow analysis, where a Spalart-Allmaras turbulence model was used to compute an unsteady RANS simulation that was later averaged in time. Their stability analysis therefore considered the linearisation of the turbulence terms of the Spalart-Allmaras model, obtaining a good agreement with the experimental results of Parezanovic and Cadot. Mettot et al. explored two different approaches, to evaluate the influence of the eddy viscosity on the analysis. First, they computed a turbulent 3D simulation of the D-shaped cylinder, and obtained a mean flow from the averaging of the solution both in time and the spanwise direction. Their linear operator (the linearised Jacobian of the flow solution) did not contain turbulence terms, and was based solely on the molecular viscosity $\mu$ (a "laminar Jacobian"). To complement it, they also evaluated the stability of the flow considering a Jacobian matrix in which the turbulence model was considered in the linearisation ("turbulent Jacobian"), but using a frozen-eddy viscosity approach, in which the eddy viscosity (obtained from the time-averaging of a SAS $k-\omega$ model) remains constant when linearising the equations. The two analysis showed excellent agreement with the experimental results.

In this section we reproduce a two-dimensional analysis of the turbulent flow around a D-shaped cylinder, using the standard version of the Spalart-Allmaras turbulence model[136] to calculate the eddy viscosity. A structured D-type mesh of approximately 200000 elements is used to discretise the flow region around a Dshaped cylinder, with a circular leading edge of diameter $D$ (Fig. 4.11). Behind the leading edge, the body is extended with a rectangular region of length $1.5 D$, ending in a blunt base region. The boundary layer mesh resolution is refined to keep $y^{+}$values lower than 1 over the body surface. Special attention is put on the 

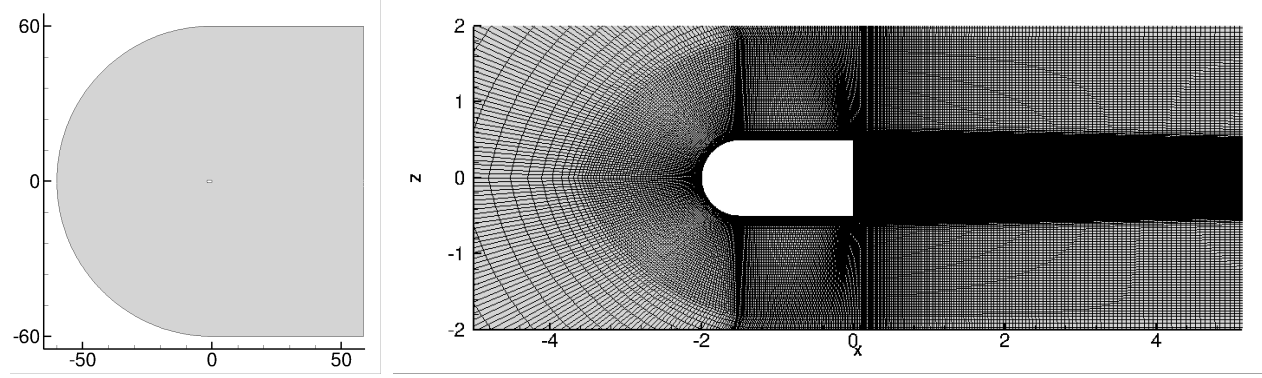

Figure 4.11: Domain distribution and close-up of the structured mesh used on the calculation of the turbulent flow around the D-shaped cylinder.
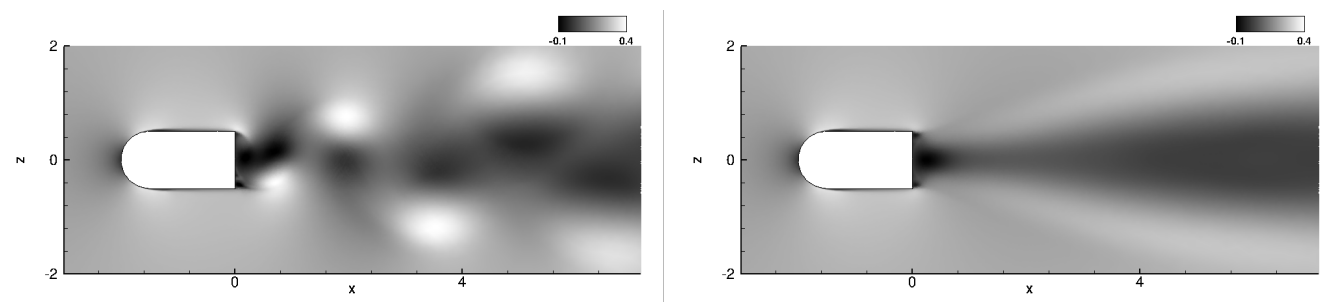

Figure 4.12: Unsteady RANS results for $R e=13000$. Streamwise velocity contours are collected for an aleatory time snapshot (left) and for the time-averaged solution (right).

refinement of the base region and wake area, and in the frontal stagnation area. A turbulent mean flow is calculated by averaging 200 periodic wake oscillation cycles, and used to extract the linear operator for the stability analysis. The frequency predicted by the analysis is correlated with the results of the URANS simulation. Finally, the eigenvalue sensitivity to a steady forcing is obtained and compared with the numerical results of Mettot[90].

\subsubsection{Results}

The dual time-stepping flow solver is set for a Reynolds number of $R e=\rho U_{\infty} D / \mu=$ 13000, fixing the free stream Mach number to $M=0.2$. The unsteady flow is dominated by self-sustained oscillations of the body wake, with alternating eddies detaching from the base region (Fig. 4.12). The wake periodicity has a frequency of $14.28 \mathrm{~Hz}$, corresponding to a Strouhal number of $S t=0.21$, in good agreement with experimental value of $S t=0.22$ measured by Parezanovic and Cadot[101]. The averaging of the URANS solution permits the extraction of the turbulent Jacobian, based on the linearisation of the eddy viscosity modelled by the Spalart-Allmaras turbulence model. As described on Section 3.2, we calculate a reduced set of eigenpairs of the generalized eigenvalue problem using a Krylov iterative solver, imposing a shift value on the spectral transformation close to the expected associated frequency, $\sigma_{i}=2 \pi S t$. A moderately unstable global mode is recovered (Fig. 4.14-(a)), 

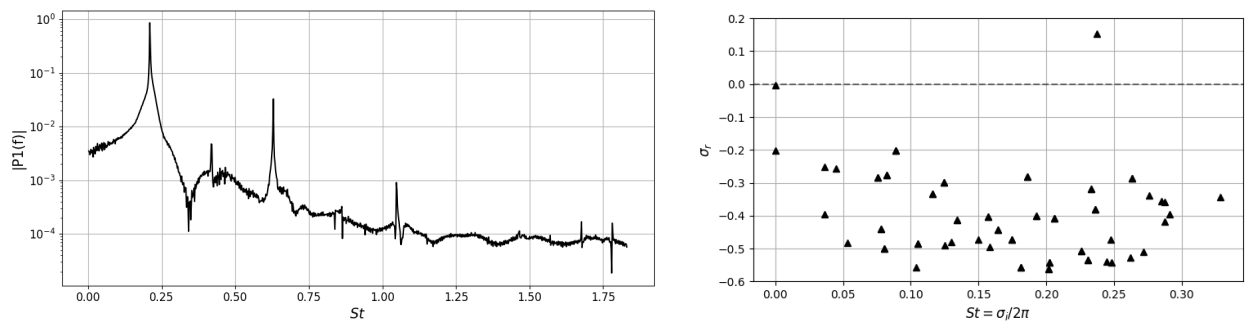

Figure 4.13: Flow oscillator main frequency obtained from the URANS simulation (left) and the Linear Stability Analysis (right).

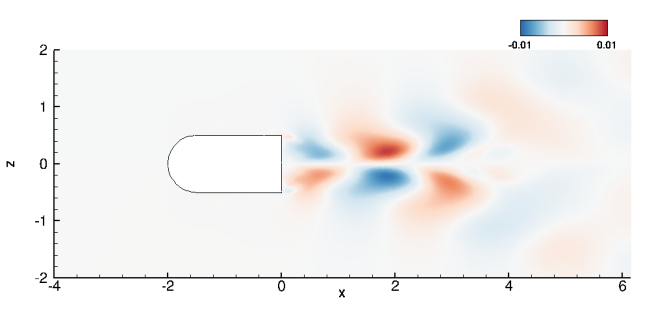

(a) Direct global mode, present study.

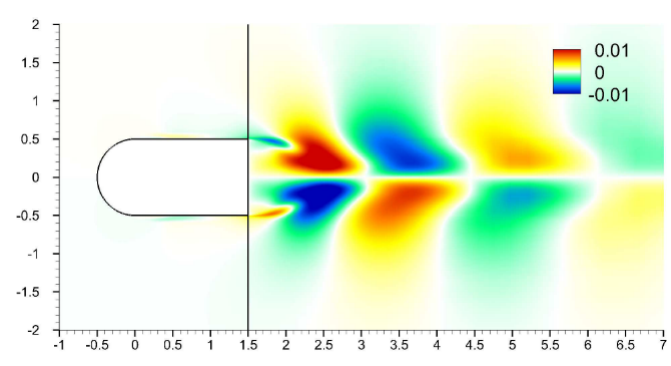

(c) Direct global mode, reference.

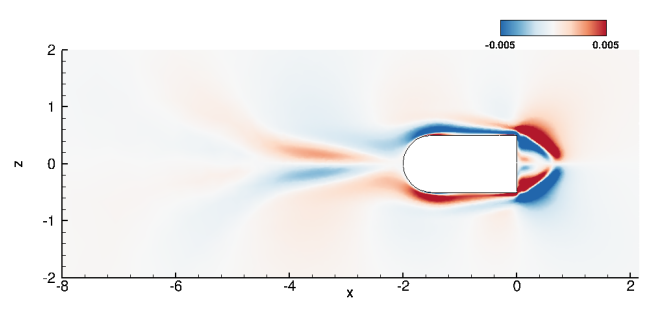

(b) Adjoint global mode, present study.

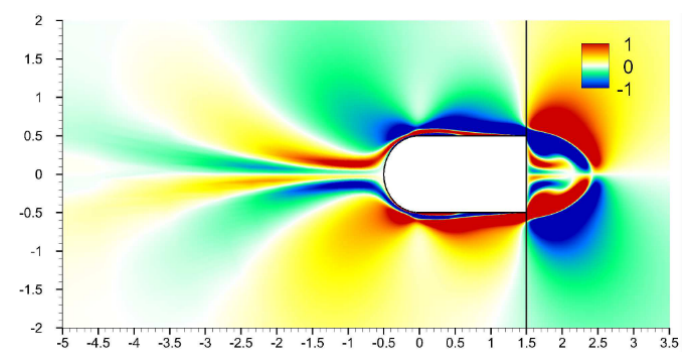

(d) Adjoint global mode, reference.

Figure 4.14: Global direct (left) and adjoint (right) modes. Figures show the spatial distribution of the real part of the stream-wise velocity components. Reference figures extracted from Mettot[88].

which structured resembles the Kevin-Helmholtz spatial distribution of the perturbations, characteristic of oscillating wake flows. The adjoint global mode intensity appears concentrated within the recirculating area (Fig. 4.14-(b)), with its maximum values near the shear layers area, but also extending upstream through the boundary layers and in the direction of the incoming flow. The results are compared with those of Mettot[88], showing excellent agreement.

Table 4.3 offers a comparison of the Strouhal values obtained by previous studies and in the present analysis. Differences between URANS solutions are small and can be assumed to be caused by the different flow solvers. The frequency predicted by the stability analysis differs from the experimental and numerical data for all the cases in the literature. This has been explained by the strong resonance 


\begin{tabular}{lcc} 
& $S t_{U R A N S / E X P}$ & $S t_{L S A}$ \\
\hline \hline Meliga et al.[86] & 0.23 & 0.25 \\
Mettot et al.[88] & 0.23 & 0.26 \\
Parezanovic and Cadot[101] & 0.22 & {$[-]$} \\
Present study & 0.21 & 0.23
\end{tabular}

Table 4.3: Characteristic frequency of the oscillating wake of the D-shaped cylinder, $R e=13000$. Data from URANS or experimental analysis are compared against Linear Stability Analysis results.

that occurs with the harmonics of the global mode[86], also responsible for the nonneutral value of the real part of the eigenvalue on the mean flow analysis. For the present study, these has been reflected in Figure 4.13, where the frequency spectrum obtained from the URANS simulation highlights the frequency of the vortex shedding and its harmonics.

The sensitivity analysis provides the proportional drift of the eigenvalue when changes in the base flow or a steady forcing are applied. The combination of direct and adjoint modes recovers the structural sensitivity of the global mode, depicted in Figure 4.15 together with sensitivity to base flow modifications. The global mode appears as most sensitive to base flow changes near the base region wall, and at the dowstream limit of the recirculation area, the most sensitive area forming an arc shape. The results of the sensitivity analysis of the imaginary part of the eigenvalue to steady forcing are compared with those of Mettot et al.[90], but however we observe different sign on our sensitivity gradients in the near wake area. The flow appears most sensitive inside the cylinder wake, but it can be also observed that perturbations upstream the cylinder will have a positive effect on the frequency of the global mode. The differences may come from a different spatial distribution of the adjoint mode due to different numerics and implementation. Also, we argue that Mettot et al. show the real part of the sensitivity gradient when referring to the sensitivity of the imaginary part of the eigenvalue, and our results are still close to the sensitivity obtained by Parezanovic and Cadot[101], where it was shown that a force in opposite direction to the incoming flow would lower the associated frequency of the wake oscillations. To confirm this fact, we calculate the impact of a control cylinder into the flow frequency, following the approach of Meliga et al.[86]. To do so, a local force at the coordinates $(x, z)$ is modeled as the drag exerted by a small cylinder:

$$
\mathbf{f}_{x z}=-\frac{1}{2} d C_{d} \mathbf{U}_{x z}|| \mathbf{U}_{x z}|| / \Omega_{x z}
$$

with $d$ and $C_{d}$ being the diameter and associated drag coefficient of the modeled control cylinder, $\mathbf{U}_{x z}$ the mean flow velocity vector at the coordinates $(x, z)$ and $\Omega_{x z}$ the associated cell volume. The sensitivity map is calculated by the inner product of the imaginary part of the sensitivity to steady forcing and the modeled forcing, $\nabla_{\mathbf{f}_{x z}} \sigma=<\nabla_{\mathbf{q}_{f}} \sigma_{i}, \mathbf{f}_{x z}>$, which result indicates the variation of the frequency of 


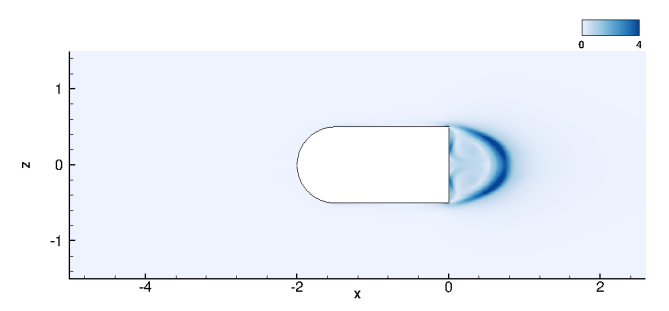

(a) Structural sensitivity.

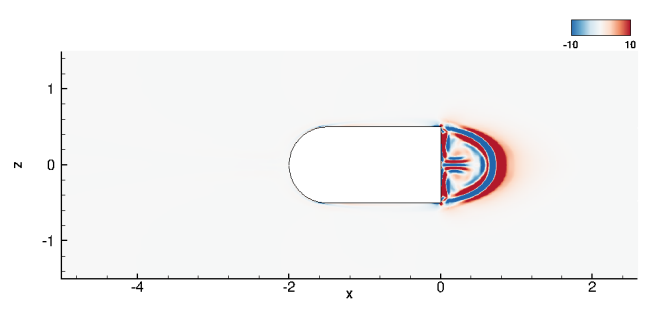

(b) Sensitivity to base flow modifications, streamwise component.

Figure 4.15: Sensitivity analysis of the leading global mode.
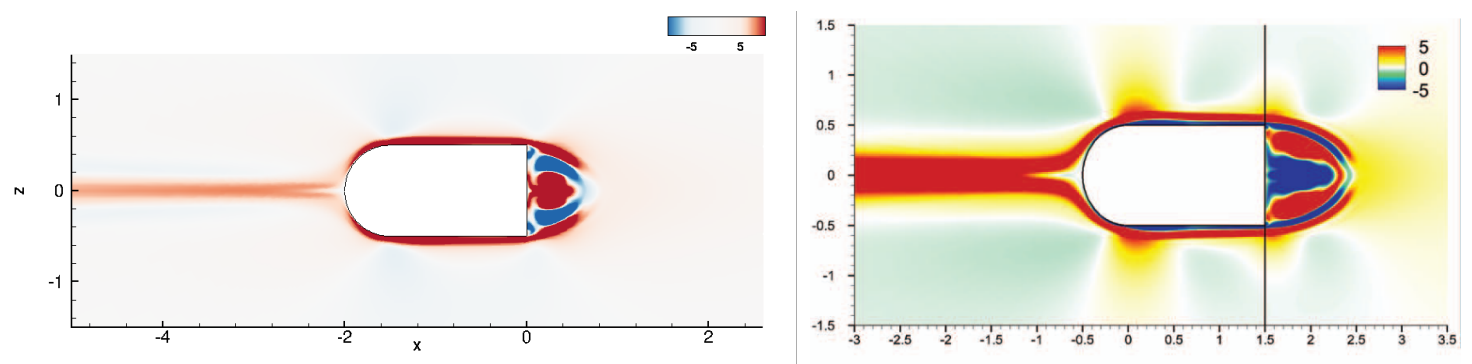

Figure 4.16: Streamwise component of the imaginary part of the eigenvalue sensitivity to a steady force. Comparison of the present results (left) with the obtained by Mettot et al.[90], reprinted with the permission of AIP Publishing.
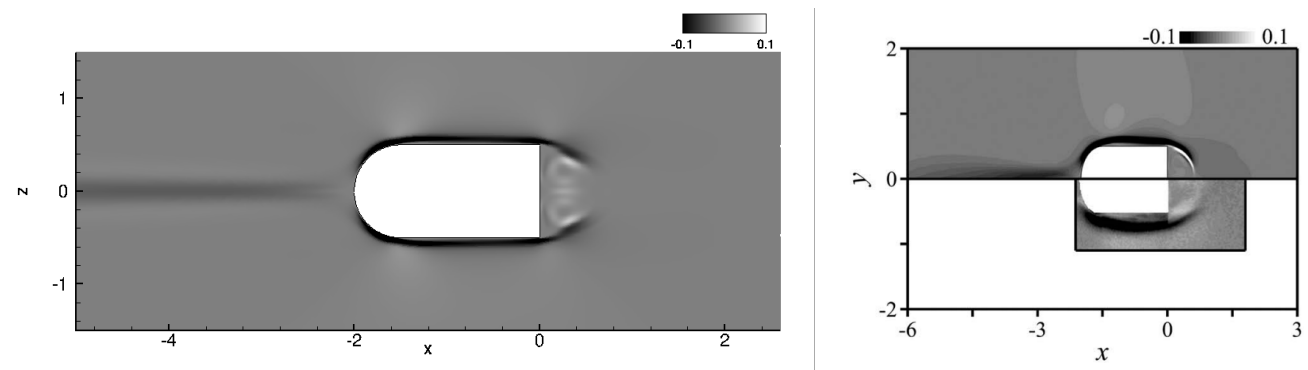

Figure 4.17: Sensitivity of the leading global mode to passive flow control. Comparison of the present results (left) with the numerical solution obtained by Meliga et al.[86] (upper half) and the experimental data of Parezanovic and Cadot[101] (lower half), reprinted with the permission of AIP Publishing.

the associated global mode generated by the presence of a control cylinder. The results are compared with both the numerical results of Meliga et al.[86] and the experimental results of Parezanovic and Cadot[101], in Figure 4.17, showing an excellent agreement. Variations predicted upstream the body and near the wall are well captured, with few variations in the wake area, where even the effects in the shear layers are well defined. 



\section{Part III}

Analysis of High Speed Flows 



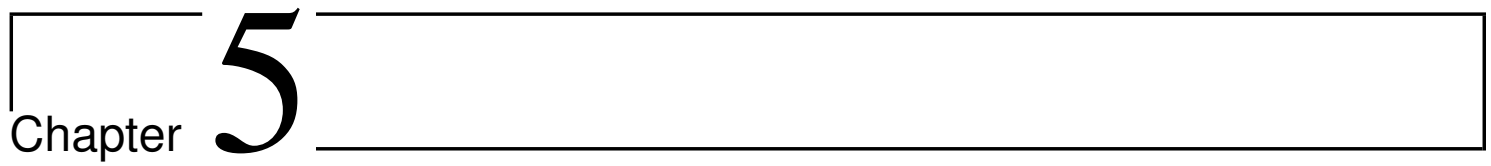

\section{Global Stability of a Base Bleed Turbine Blade Trailing Edge}

\section{Contents}

5.1 Instability detection and characterisation . . . . . . 58

5.1 .1 Introduction . . . . . . . . . . . . 58

5.1 .2 Numerical procedure . . . . . . . . . . . . . . 61

5.1 .2 .1 Base flow solver . . . . . . . . . . . . 61

5.1.2.2 Domain Reduction analysis . . . . . . . . . 64

5.1 .3 Results ...................... 64

5.1.3.1 Flow topology analysis . . . . . . . . . 64

5.1.3.2 Stability analysis . . . . . . . . . . . 67

5.1.3.3 Sensitivity analysis . . . . . . . . . 70

5.1 .4 Conclusions . . . . . . . . . . . . . . . 73

5.2 Further Investigation . . . . . . . . . . 75

5.2 .1 Introduction . . . . . . . . . . . . . 75

5.2.2 Experimental methodology and model description . . . . 76

5.2 .3 Numerical experiments . . . . . . . . . . . . . . . . 77

5.2 .4 Flow characterization . . . . . . . . . . . . 79

5.2.4.1 Subsonic flow . . . . . . . . . . . . . . . 79

5.2 .4 .2 Transonic flow . . . . . . . . . . . . . 82

5.2.4.3 Supersonic flow . . . . . . . . . . . . . 83

5.2 .5 Base region flow sensitivity . . . . . . . . . 85

5.2 .6 Conclusions and way forward $\ldots \ldots \ldots . \ldots 8$

In this chapter, the direct and adjoint methods for stability analyses are applied to a realistic aerospace configuration, a turbine blade trailing edge. The chapter has been divided in three sections, corresponding to three different indexed publications. The first section, where the global instability is first detected and characterized, was 
published on the AIAA Journal under the title "Pressure Bifurcation Phenomenon on Supersonic Blowing Trailing Edges"[82], and later expanded on the Proceedings of 7th European Conference on Computational Fluid Dynamics (ECFD 7) under the title "Sensitivity analysis of supersonic turbine trailing edges"[81]. The second and last section of the chapter was carried out in collaboration with the PETAL group, of Purdue University, as the numerical analysis of a wind tunnel model where the effects of base bleed will be studied. The results of the analysis were presented at the AIAA SciTech Forum 2019, under the Conference Proceedings entitled " Coanda Flow Characterization on Base Bleed Configurations Using Global Stability Analysis" [79].

\subsection{Instability detection and characterisation}

\subsubsection{Introduction}

Air transport has experienced a very large growth in last decades, with a significant socio-economic relevance for integration and development at regional and international level. From a technological point of view, the aerospace industry is constantly at the cutting edge of scientific development. Continuous improvements in aerodynamics, material sciences, manufacturing processes, avionics, control and navigation systems make aircraft more efficient and safer every day. In particular, aircraft engines tend towards more compact architectures, the structural safety limits are tighter and aero-structural cou-

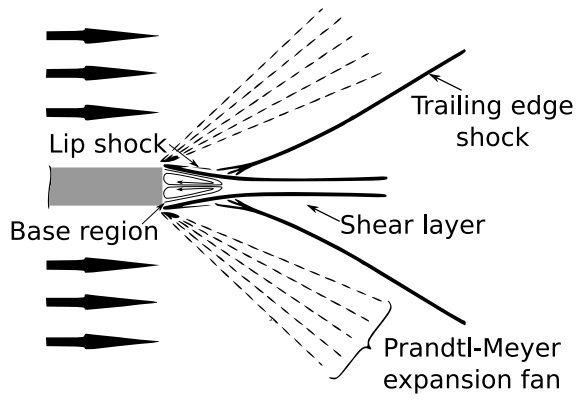

Figure 5.1: Schematic representation of supersonic flow on a blunt trailing edge. Image modified from Saracoglu et al.[122] plings may lead to low and high cycle fatigue issues. Such aero-structural interactions are significantly detrimental when the internal flow velocities approach the speed of sound. Engine turbine blades can operate in transonic or supersonic flow regime, specially over high pressure turbine passages where the flow expand to supersonic velocities experiencing complex shock wave systems at the trailing edge. These waves can interact with downstream stages of the turbine affecting the aerodynamic efficiency of the blades and reducing the life-span of the engine component[100]. Up to $30 \%$ of the total loses on transonic turbines are due to these shock interactions[32]. Part of the aero-structural forces coupling have their origin on the flow fluctuations that take place at the area adjacent to the trailing edge, known as the base region. This zone is characterized by low momentum and constant pressure, having a strong influence on several features of the surrounding flow field. The evaluation of the parameters driving the compression and expansion waves, shear layers and vor- 


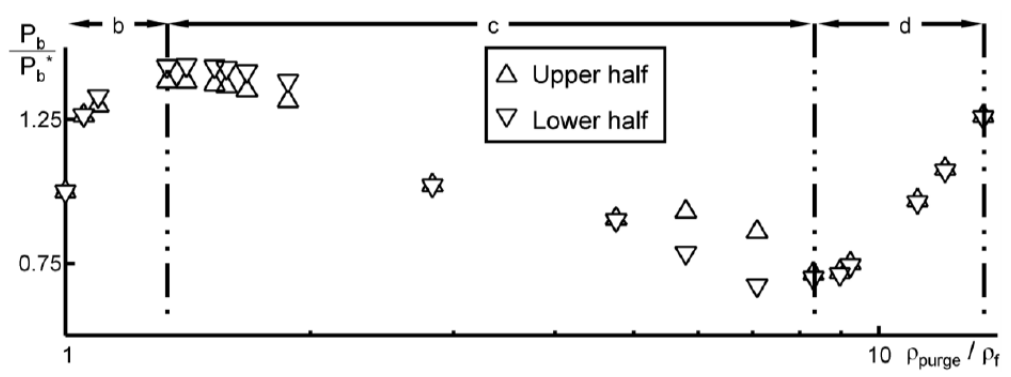

Figure 5.2: Base pressure correlation at the trailing edge as a function of the density ratio of the bleeding rate. Image reprinted from Saracoglu et al.[122].

tex shedding phenomenon should then allow us to mitigate their unsteady couplings with the turbine blades.

Figure 5.1 summarizes the flow topology of a supersonic trailing edge of a turbine blade, where the main flow features are schematically represented. The upstream boundary layers from suction and pressure sides remain attached at the trailing edge corner and separate in an alternate manner, characterized by the Strouhal number, developing a pair of shear layers that lead to the formation of two (or more, depending on the Reynolds number) families of vortices, while, simultaneously, the main flow accelerates through a Prandtl-Meyer expansion fan. This unsteady flow separation is a source of tonal noise, also causing mechanical and thermal fatigue not only on the body of origin but on the ones situated downstream, affected by the travelling vortices and unsteady shock waves. The upper and lower shear layers propagate downstream and eventually merge at the wake on a point of confluence, creating a dead air zone limited by both shear layers and the blunt trailing edge, namely the base region. At the confluence point, the flow changes its direction notably, being forced to compress through a system of strong trailing edge shocks. The degree of compression, hence the strength of the shock waves, highly depends on the base region properties. Furthermore, a weak compression wave, called separation or lip shock, is formed at the point of flow detachment in order to adapt the pressure gradient between the shearing and the downstream flow. The base region has been an object of study for long time [51, 93, 94, 95], and its influence and characteristic topology on turbine cascades $[32,131]$ or the supersonic turbulent wake of axisymmetric bodies $[55,117,118]$ repetitively revisited.

The thin trailing edge of turbine blades cannot be protected using internal cooling passages, so cooler flow bled from the high pressure compressor is purged through slots or orifices to avoid the melting of the turbine components. As it has been shown by several authors[107, 121], this cooling could be exploited to modulate the base region properties. Different studies[15, 68, 154] have been devoted to examine the effects of trailing edge bleeding on the base region. Of particular importance, Saracoglu et al.[122] investigated the flow topology for a supersonic blunt trailing edge as a function of the cooling flow intensity, showing the dependence 
of the base pressure with the blowing magnitude. A relevant result of the study was the presence of non-symmetric configurations at the base flow (shock intensity and angle of the shock wave) for a specific range of blowing rates (Figure 5.2). Two different bifurcations from symmetric to non-symmetric configurations were observed, the first one arising when the base pressure is close to reach its maximum value, and a second one appearing for higher blowing ratios, close to a local minimum value for the base pressure.

In this chapter we combine RANS simulations and linear stability theory to explain the mechanisms that generate the described non-symmetric configuration. Only the first pressure bifurcation is analysed in detail, focusing on the area where the purge flow provokes an important increment on the base pressure, reducing the intensity of the shock waves and the base pressure losses. A thorough analysis of the flow topology of a supersonic blowing trailing edge is carried, focusing on the recirculation areas of the base region, showing how those areas interact generating changes on the injected flow and therefore in the complex wave system. Those changes are then also identified using global stability analysis. Stability analysis studies the growth or decay of perturbations superimposed upon a steady base flow, and has proven to be effective in the analysis of incompressible and compressible flows, either in laminar or turbulent configurations. The analysis aims to identify which features can naturally appear in the flow and become dominant for some particular value of the parameters. Depending on the nature of the phenomenon, the analysis can be performed over a steady base flow solution[61, 62, 123], over an averaged mean flow [11, 98, 132], or using time-stepping or DMD techniques for the study of transient phenomena[48, 69]. On this work, steady base flows were considered to characterize the stability behaviour of the system. The results of this study shed some light on the pressure bifurcation detected by Saracoglu et al.[122], identifying the physical global mode responsible of the non-symmetrical conditions as a function of the cooling flow purge intensity. We anticipate that results of the stability analysis match the bifurcation predicted by RANS simulations, adding extra information about the underlying physical mechanisms. The unstable configuration seems to be related to the sudden geometrical expansion of the flow at the end of the cooling slot. The associated instability forces the purge flow direction to deflect and impact one of the shear layers, generating an additional shock wave system. Such changes on the vicinity of the trailing edge would have a great effect on the loads affecting adjacent blades and turbine stages. The identified global mode changes its shape with the blowing rate intensity, as does the flow topology of the base region, giving the stability analysis valuable information about where the perturbations are located.

The rest of this section is organized as follows. Section 5.1.2 summarizes the numerical procedures to obtain the base flow and stability solutions. In section 5.1.3 the main results are described, to finish in Section 5.1.4 with the main conclusions of this study. 


\subsubsection{Numerical procedure}

\subsubsection{Base flow solver}

The domain of reference of the problem (see Figure 5.3) had been previously studied by Saracoglu et al.[122] and Gorbachova et al.[47]. The former used extensive URANS simulation to give a comprehensive analysis of the effects of the trailing edge purge flow on the flow topology, and the latter did a first approach to the problem throughout global stability analysis. The geometrical approximation was a simplification of the industrial problem, where only the details of the flow in the vicinity of the trailing edge of a turbine blade were studied. The geometry also included the internal cooling passages, composed by a cavity plenum, where the purge flow was ejected, and the injector pipe, directly communicating the plenum with the base region.

The computational domain was limited by straight boundaries, with a length of $21.75 \times d$ (with $d$ as the trailing edge thickness, equal to $0.02 \mathrm{~m}$ ) and a width of $20 \times d$. The injector pipe had a length and width of $2.5 \times d$ and $0.3 \times d$, respectively. To keep subsonic conditions inside the cavity plenum, its width was kept three times larger than the injector pipe. The characteristic geometrical lengths of the analysis are shown in Table 5.1.

Boundary conditions of the control volume were defined as follows: Supersonic inflow and output, Euler wall and symmetry plane were set at left, right, upper and lower limits respectively; non-slip wall was used for all internal surfaces and a reservoir-pressure inflow boundary condition was set to simulate the input of the cooling in the cavity plenum area (Fig. 5.3).

The Mach number at the inflow boundary was set to 1.5, keeping a total temperature of $365 \mathrm{~K}$ and a Reynolds number of $9.4 \times 10^{6}$, based on the trailing edge length $(11.65 \times d)$. The flow rate at the cooling flow exit, from now on referred as the blowing rate, was defined by the cooling flow static temperature, kept to 300 $\mathrm{K}$, and its total pressure, expressed as a percentage of the free stream flow total pressure.

Table 5.1: Fluid domain dimensions

\begin{tabular}{lc}
\hline \hline Boundary & Length $(\mathrm{m})$ \\
\hline \hline Domain length & 0.4346 \\
Domain height & 0.4 \\
\hline Trailing edge length & 0.233 \\
Trailing edge height & 0.02 \\
\hline Cavity plenum length & 0.05 \\
Cavity plenum height & 0.018 \\
\hline Injector channel length & 0.05 \\
Injector channel height & 0.006 \\
\hline \hline
\end{tabular}




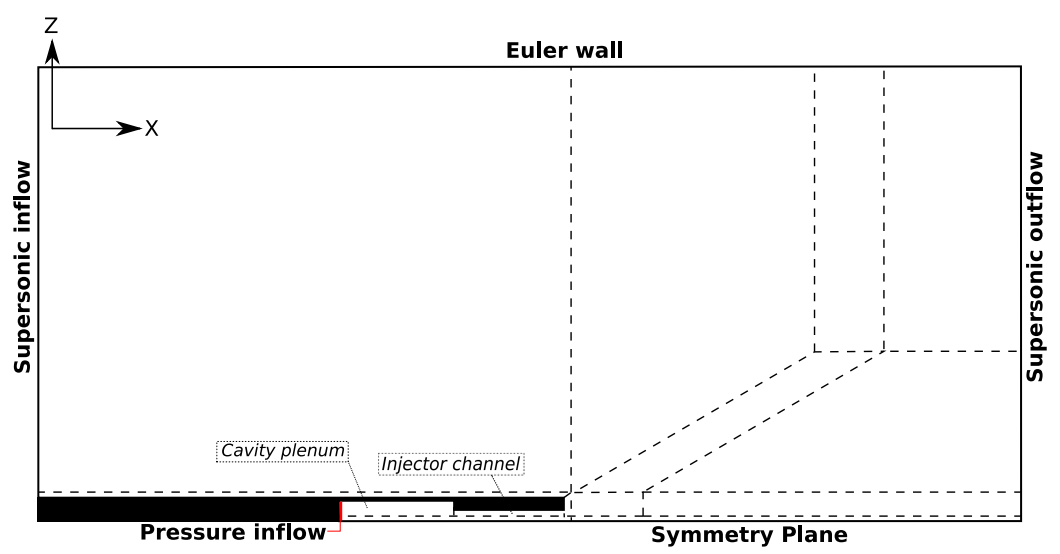

Figure 5.3: Geometry definition (solid) and grid topology (dashed-lines) used on the mesh generation. Only half domain is shown for simplification.

Since the study aimed to understand the different flow features present in the flow and, in particular, the nature of the non-symmetry behaviour, a global stability analysis over the steady symmetric base flow was performed. Thus, only half of the domain was simulated using a RANS approach, imposing a symmetry boundary condition on the plane of symmetry to obtain a steady solution. After obtaining a "half" base flow, mesh and solution were mirrored into the symmetry plane and the stability analysis of the two-dimensional full domain was carried out. The global analysis of the mirrored-full domain around this symmetric flow at different blowing ratios will show non-symmetric modes in the analysis, which could eventually grow and generate a new non-symmetric flow configuration. To clarify the exposition, when the term half-domain is used on this text, will refer to the mesh of Figure 5.4, with a symmetry plane on its lower boundary; on the contrary, when full-domain is called, it will refer to simulations done on a mirrored mesh, considering upper and lower surfaces of the trailing edge. Similar methodology on the detection of flow bifurcation can be found on the literature on the detection of global modes of symmetric sudden expansions[37] or X-junction flow configurations[69].

The domain was discretised using a quad-structured mesh, generated using an O-Grid topology and dividing the geometry into regions of interest. Wall normal grid distances were set small enough so values of $y^{+}$lower than 1 were kept on the body surfaces, and all the scales of the boundary layer were fully resolved without the use of any wall model. Grid topology and mesh details are pictured on Figures 5.3 and 5.4. The mesh spatial resolution was concentrated at the contraction-expansion and on the shock wave areas.

To ensure mesh independent results, a mesh convergence study was performed for a blowing rate of $18 \%$ for a half-domain configuration. Six different meshes of increasing number of elements were defined, named from M1 to M6. A systematic refinement was performed to evaluate the flow sensitivity to the mesh density. At each step the mesh was refined increasing $n$ times the number of nodes, taking the 


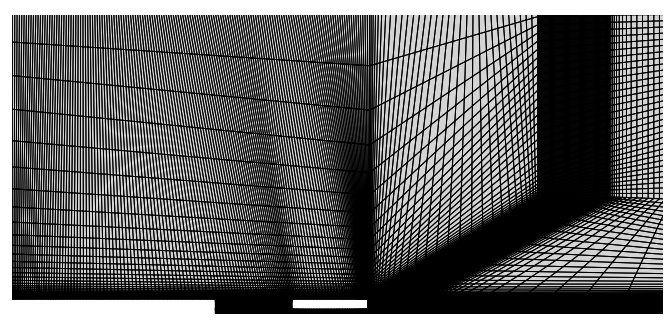

(a) Computational mesh

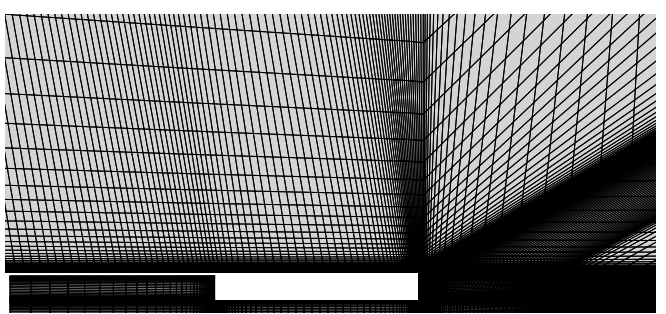

(b) Details of the mesh in the of the cavity and channel area

Figure 5.4: Computational mesh. Only a quarter of total nodes are represented for clarity.

Table 5.2: Mesh Convergence Analysis performed for a blowing rate of $18 \%$ for a half-domain configuration. $P_{b} / P_{b}^{*}$ represented the relation between the pressure with and without purge flow applied, and $\rho_{\text {purge }} / \rho_{f}$ was the relation between the density values at the cavity plenum and at the base region.

\begin{tabular}{lccc}
\hline \hline Mesh & Number of nodes & $P_{b} / P_{b}^{*}$ & $\rho_{\text {purge }} / \rho_{f}$ \\
\hline M1 & 12010 & 1.26932202 & 1.03157676 \\
M2 & 48260 & 1.29883128 & 1.03038449 \\
M3 & 104630 & 1.30545566 & 1.02675552 \\
M4 & 207106 & 1.31826117 & 1.01727924 \\
M5 & 321090 & 1.31929642 & 1.01407829 \\
M6 & 424640 & 1.31858961 & 1.01426252 \\
\hline \hline
\end{tabular}

mesh M1 as the reference. This refinement law preserved the topology of the mesh and guaranteed that Richardson extrapolation formulas were applicable to obtain an accurate rate of convergence[42]. The convergence criteria, evaluated through the global density residual $\mathrm{res}_{\rho}^{n}$, was set up to values lower than $10^{-6}$. It was defined as:

$$
\left\|r e s_{\rho}^{n}\right\|=\sqrt{\sum_{j=1}^{N} \frac{\left[r e s_{\rho}^{n}(j)\right]^{2}}{N}}
$$

where $N$ denoted the number of grid points.

Detailed results from the mesh convergence study are shown in Table 5.2. At each mesh the pressure and density values at the trailing edge were monitored. According to these results, the mesh M4 was selected as a good compromise between accuracy and computational efficiency. 


\subsubsection{Domain Reduction analysis}

The use of a reduced domain on the linear stability analysis can avoid boundary condition interactions, reduce the size of the considered linear operator and improve the convergence of the iterative method to the desired eigenvalues. On the case of study, the region of interest was concentrated around the base region and the cavityinjector area (Fig. 5.5-(b)), representing a small section of the whole computational domain. To eliminate part of the contribution of the large expansion and compression regions and to reduce the size of the linear operator considered for the stability analyses, an evaluation of the effects of reducing the domain size is performed.

In order to illustrate the influence of the domain used on the stability analysis, part of the eigenspectrum for a blowing rate of $18 \%$ is shown in Figure 5.5-(a). The abscissa represents the number of requested eigenvalues (NEV), set up as a third of the projected Krylov subspace size (NCV), and the ordinate shows the real part of the least stable eigenvalue, $\omega_{r}$. Three different domains, denoted as DR1 to DR3, were considered and compared to the results obtained without any domain reduction technique (Fig. 5.5-(b)). The tendency of the results are in good agreement with the one predicted by Sanvido et al.[119], where the eigenvalue showed a strong sensitivity to the domain extension. It is not the aim of this work to explain the reason of these differences but only the applicability of the methodology. A complete description of the sources and estimation of the errors is given in Sanvido et al.[119]. According to these results, DR3 was used for the stability computations on the rest of this work.

The number of degrees of freedom of the reduced domain DR3 was 436320, which meant a total of $\simeq 10^{11}$ elements of the Jacobian matrix, and a number of non-zero elements of approximately $3.3 \times 10^{7}$, almost a third of the non-zero elements of the original matrix. To highlight the advantages of the use of a reduced domain, statistics calculated on a local workstation equipped with 8 IntelCore ${ }^{T M} i 7-6700 K$ CPUs and 32 GB of available RAM memory were collected. Prior to the application of the DR technique, the solve of the eigenvalue problem would take a total of 700 seconds to finalize, consuming more than 25 GB of RAM memory and with a number of non-zero elements of $8.7 \times 10^{8}$ for the factorized matrix. After the application of the domain reduction, the time to solve the eigenvalue problem was reduced to 210 seconds, demanding only 8 GB of RAM memory and with a number of non-zero elements of $2.8 \times 10^{8}$ for the factorized matrix.

\subsubsection{Results}

\subsubsection{Flow topology analysis}

The results shown here are expressed as a function of the blowing rate intensity, defined as mentioned by its static temperature and total pressure, the last defined as a percentage of the free stream total pressure. Due to its widespread use in the literature related to turbomachinery trailing edge cooling, free stream to purge density ratio $\left(\rho_{\text {purge }} / \rho_{f}\right)$ was also calculated to facilitate possible comparisons. The 


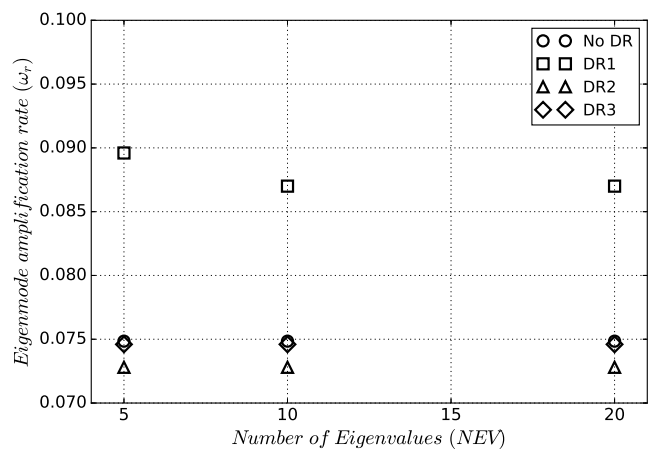

(a) Influence of the domain reduction technique on the eigenvalue analysis.

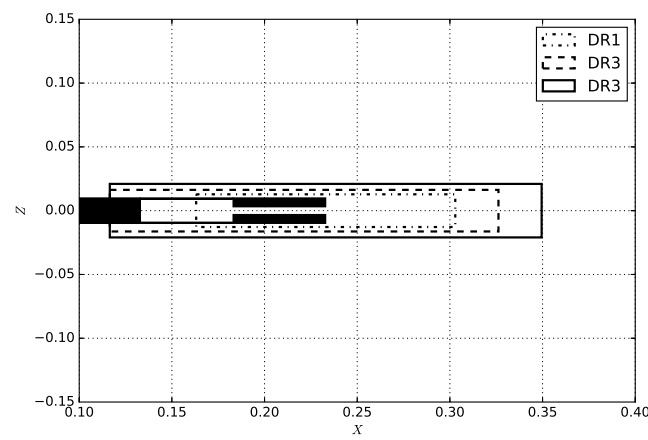

(b) Illustration of the different reduced domains used for the analysis.

Figure 5.5: Domain reduction technique convergence analysis.

free stream density value is defined as the mean density on the trailing edge faces, and the density of the purge flow as the one in the cavity plenum. The relation between these two, obtained in the direct computations of the full domain, is shown in Figure 5.6-(a). The results agreed with those obtained by Saracoglu et al.[122] and Gorbachova et al.[47]. Additionally, in Figure 5.6-(b) the ratio between the computed base pressure at both sides of the trailing edge $\left(P_{b}\right)$ and the base pressure for a non-blowing configuration $\left(P_{b}^{*}\right)$ is shown as a function of the blowing rate. Pressure values bifurcated for a blowing rate of $17.8 \%$, going back to the symmetric flow state for a blowing rate of $42 \%$ and reaching a maximum value of a $3.6 \%$ of pressure difference at $28 \%$ of blowing intensity. The value of the blowing rate for the maximum pressure difference did not match the maximum value for the base pressure, which took place at a blowing rate of $24 \%$. As it will be shown, the reason of this behaviour is the appearance of a non-symmetric global mode which eventually becomes unstable and dominates the flow configuration. With the aim of capturing the instability related with the onset of this bifurcation and identifying the sources of the non-symmetry, cases where a blowing rate went from $10 \%$ to $45 \%$ were analysed.

For illustration, the base flow pressure and velocity fields for some of the test cases obtained with the mesh M4, including the solution for a non-blowing configuration, are displayed in Figures 5.7 and 5.8. These figures reveal how the shock waves decrease its intensity for low purge intensities, which combined with the filling effect of the purge jet increases the pressure of the base region. For blowing rates values higher than $24 \%$, the flow on the dead area starts to evacuate and the pressure at the base region decays, recovering the shock part of its original strength.

The analysis of the solutions obtained with the full-mesh allowed to understand the mechanisms of the non-symmetry. A detailed evolution of the recirculation areas is shown on Figure 5.9, where only negatives velocities are plotted. As the jet is applied at the base region, the recirculation area is pushed downstream, splitting 


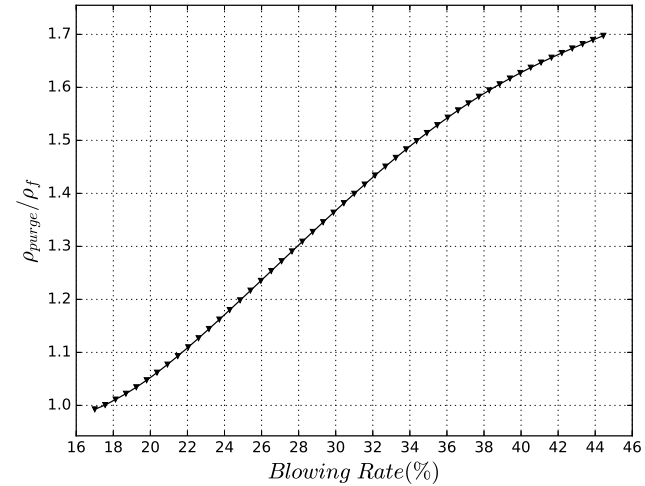

(a) Relation between the blowing rate and the density ratio.

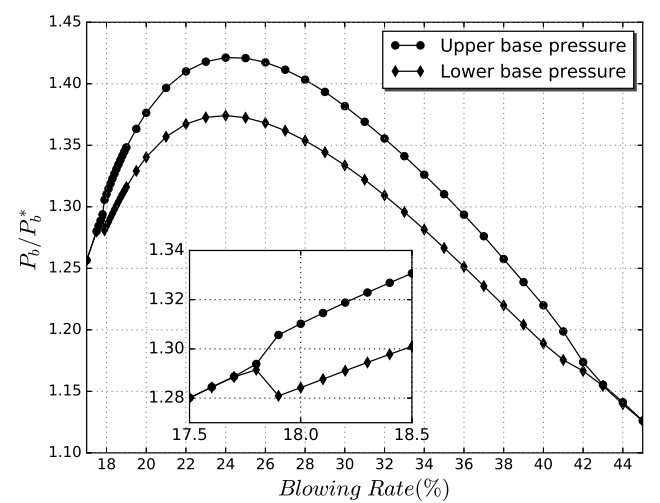

(b) Base pressure bifurcation, as a function of the blowing rate.

Figure 5.6: Density and Pressure values measured at the base region.

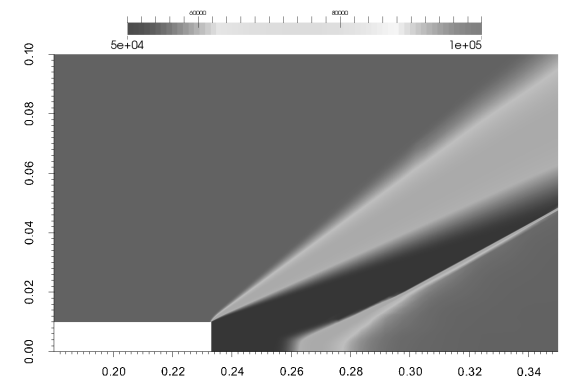

(a) No bleeding

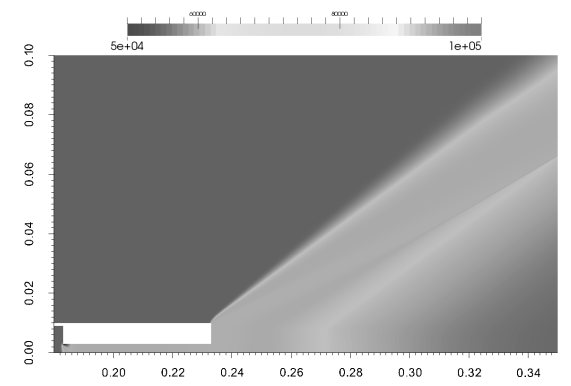

(c) $31 \%$ blowing rate

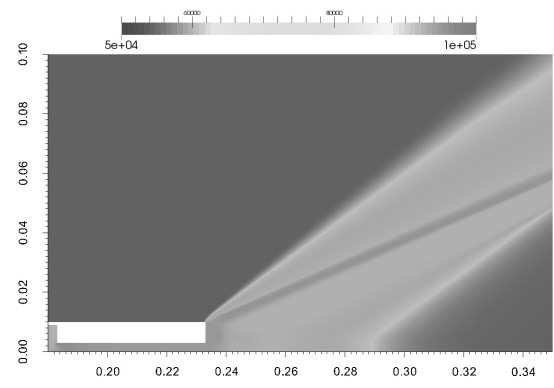

(b) $17 \%$ blowing rate

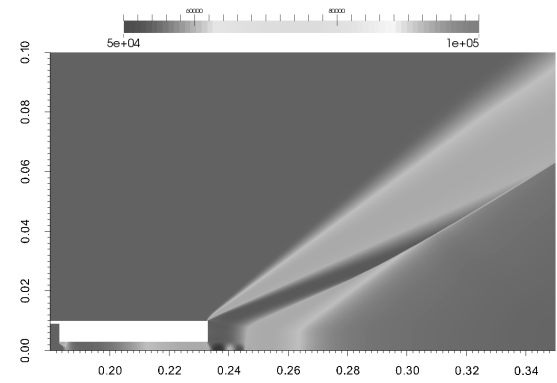

(d) $43 \%$ blowing rate

Figure 5.7: Base flow computations for different test cases and no bleeding configuration. Pressure contours.

the recirculation region in three main areas, two at the trailing edge tips and a third one downstream. However, for blowing ratios above the bifurcation point one of the recirculation bubbles of the trailing edge joins the recirculation area downstream, increasing the pressure on that side and forcing the flow to change its direction in a Coanda effect style. This change alters the wake structure, affecting the shocks 


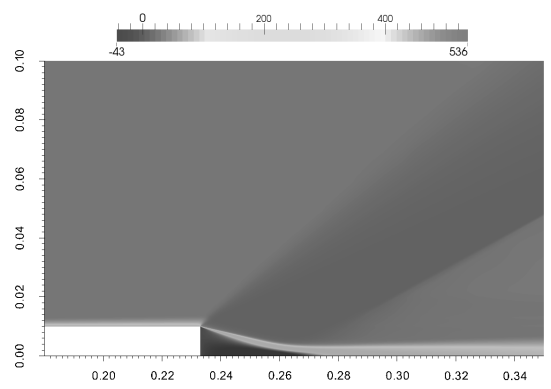

(a) No blowing

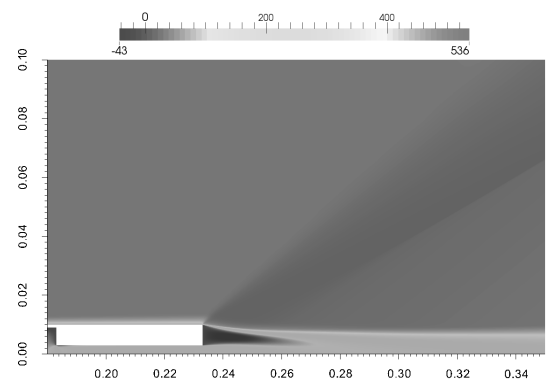

(c) $31 \%$ blowing rate

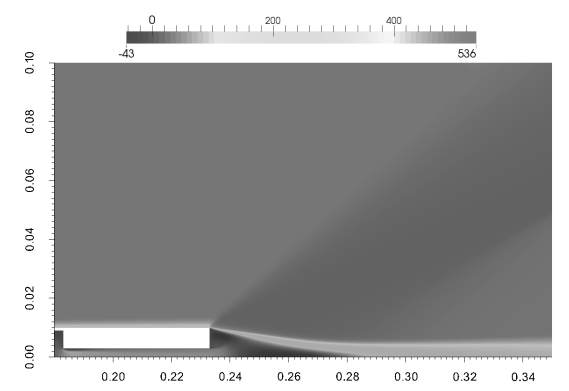

(b) $17 \%$ blowing rate

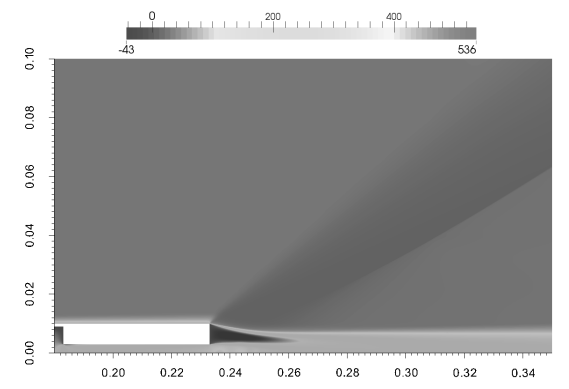

(d) $43 \%$ blowing rate

Figure 5.8: Base flow computations for different test cases and no bleeding configuration. Streamwise velocity contours.

angles and intensity. Larger blowing rates, however, stabilize the flow pushing the dead air zones towards the symmetry plane as more flow is entrained into the main jet, until the symmetric state is achieved again. The non-symmetric effect can also be appreciated from shadow-graph contour fields shown in Figure 5.10, where it is easier to identify the weakening of the trailing edge shock wave and the appearance of a secondary upstream shock only in one side of the domain. The secondary upstream shock has its foot near the region where the purging flow contacts the trailing edge shear layers, so if the flow changes its direction and deflects to the contrary side, the secondary shock would swap side as well.

\subsubsection{Stability analysis}

On Saracoglu et al.[122], the pressure measurements were done over a URANS simulation, showing an asymmetrical state that was constant in time, suggesting that the difference on pressure had not a periodic oscillation behaviour but a steady one. As shown on the flow topology analysis (Sec 5.1.3.1), the cooling flow experiments a contraction at the plenum area, followed by a sudden expansion at the base region, limited on the upper and lower sides by the shear layers. According to the type of perturbation observed on the base flow (Fig. 5.6-(a)), the geometry configuration and the results of Saracoglu[122], it would be expected to find the physical eigenvalues on the imaginary axis, having a null pulsation. This kind of instability, known 


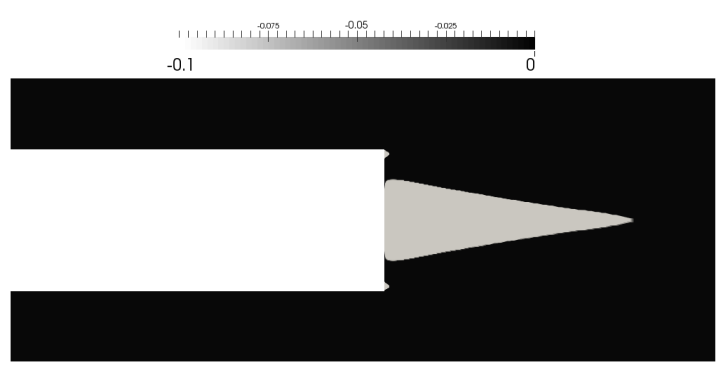

(a) No blowing

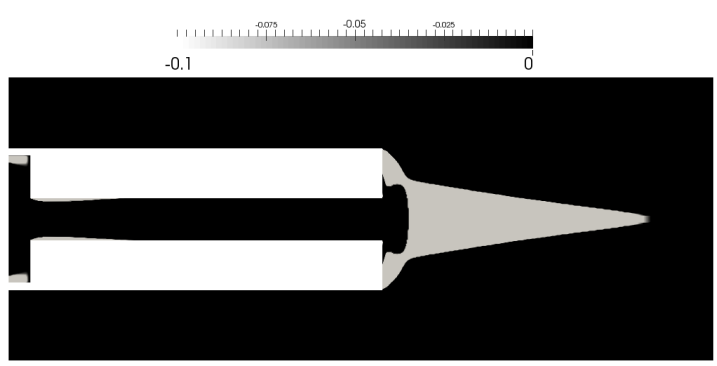

(c) $15 \%$ blowing rate

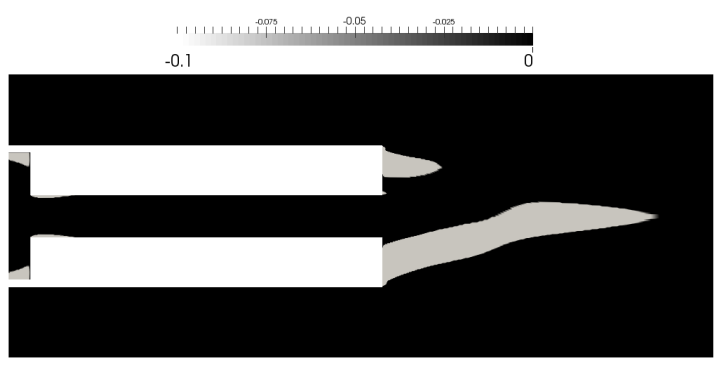

(e) $18 \%$ blowing rate

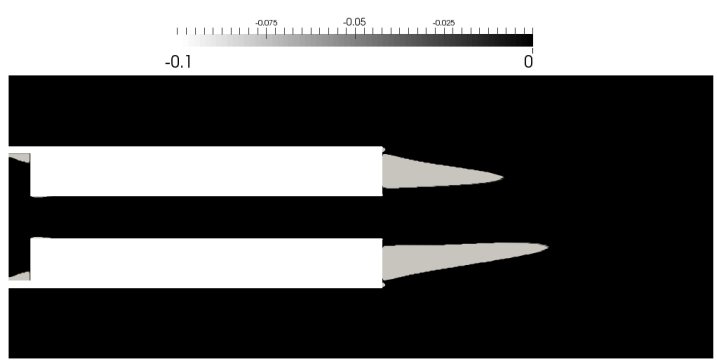

(g) $33 \%$ blowing rate

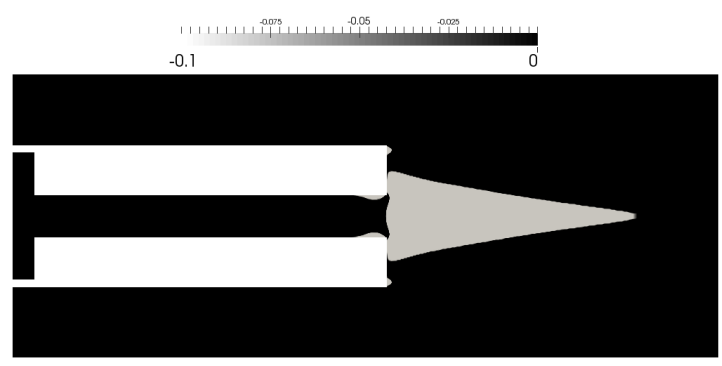

(b) $10 \%$ blowing rate

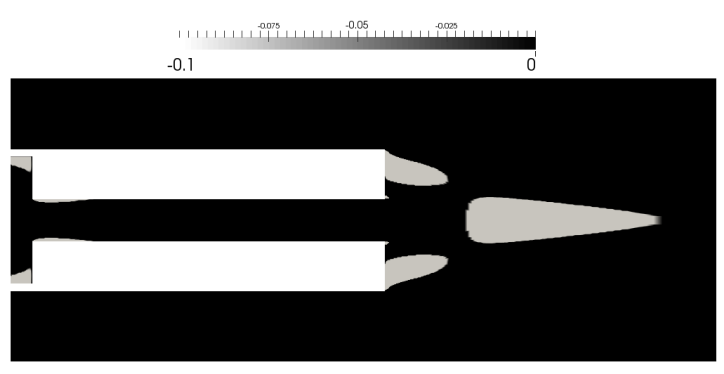

(d) $17 \%$ blowing rate

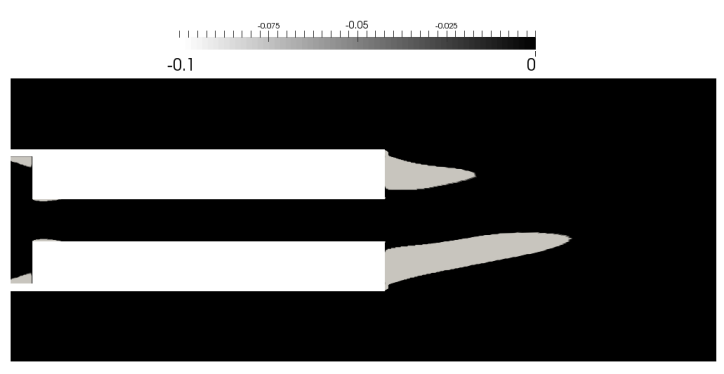

(f) $22 \%$ blowing rate

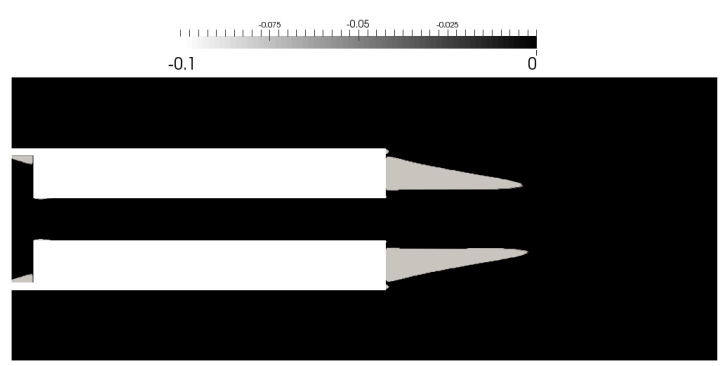

(h) $42 \%$ blowing rate

Figure 5.9: Dead air regions for different test cases and no bleeding configuration. Only negative velocities values are plotted.

as a pitchfork bifurcation, would led to a steady asymmetric behaviour of the recirculation regions at the trailing edge. Therefore, the focus was set on the region of 


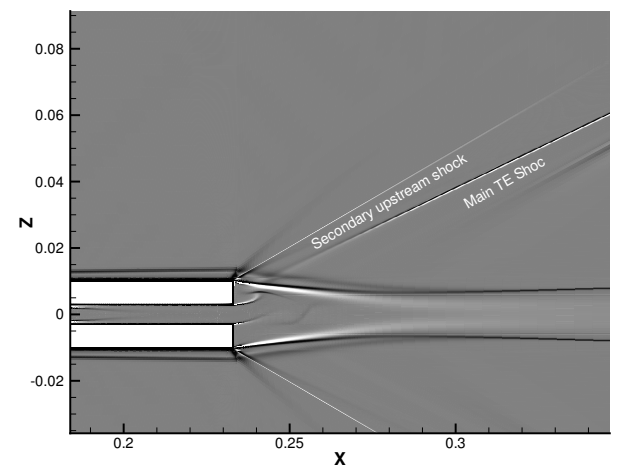

(a) $18 \%$ blowing rate

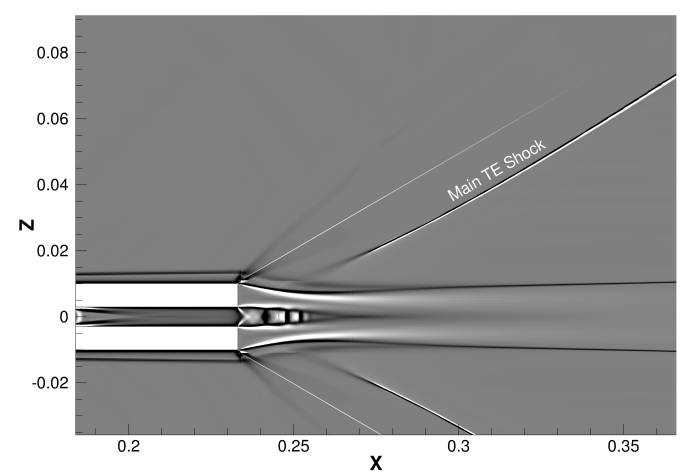

(b) $45 \%$ blowing rate

Figure 5.10: Shadowgraph contours for non-symmetric (a) and symmetric (b) flow configurations.

the spectrum close to the origin and a zero value for the shift parameter was used on the preconditioning of the Jacobian matrix.

As previously described, the base flows were computed using the half-domain mesh whereas for the stability analysis the full-domain mesh was used. All the simulations were performed using M4 mesh of the convergence study and the reduced domain DR3 for the stability analysis.

For blowing rates below $18 \%$, all the eigenvalues of the spectrum remain on the stable region. When the blowing rate is increased, however, a single anti-symmetrical mode becomes unstable and its amplification rate evolves as a function of this parameter (Fig. 5.11). This mode is directly related to the pressure bifurcation, its associate eigenvalue crossing the real axis when the purge intensity is above $18.1 \%$, and becoming stable again for a blowing rate of $38 \%$ (Fig. 5.12). When the eigenvalue evolution is related to the base flow results, it can be observed that as the base region experiments changes on its flow topology, enlarging the

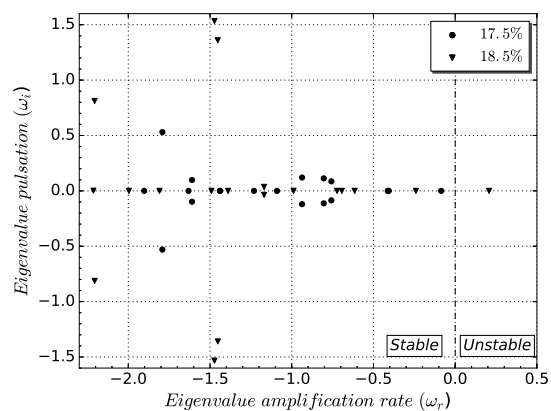

Figure 5.11: Evolution of the eigenvalue spectrum for a stable $(17.5 \%)$ and an unstable $(18.5 \%)$ configuration. recirculation bubbles of the trailing edge and showing an increment of the pressure drop at the wake (as shown in Figures 5.7 and 5.9 ), the amplification rate of the unstable eigenvalue reaches its maximum value. After this point, the eigenvalue starts its damping until a blowing rate value of $38 \%$, where it becomes stable again at the same time the base flow recovers its symmetric state. 


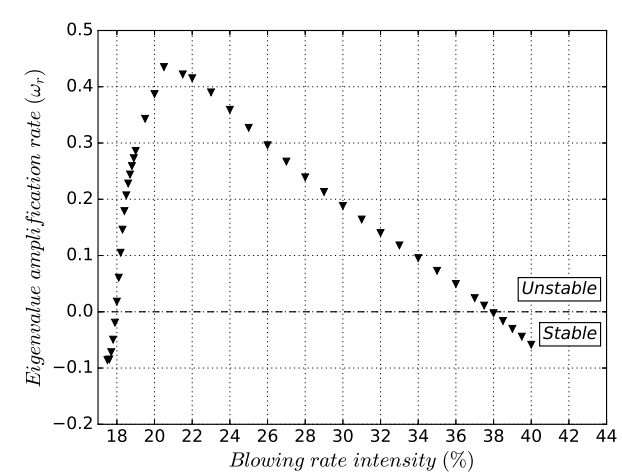

(a) Eigenvalue evolution with the blowing rate

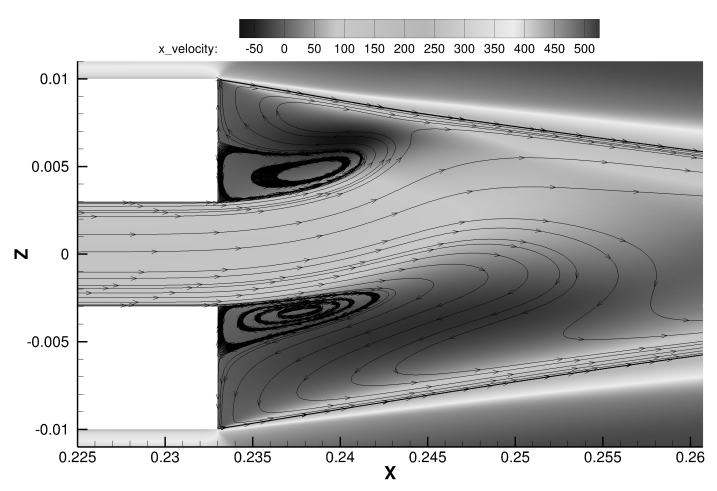

(b) Flow solution for a non-symmetric configuration

Figure 5.12: Relation between the global mode and the flow topology.

The eigenmode structure consists in two symmetrical lobe-shaped regions with anti-symmetrical streamwise perturbation components, and it changes its structure as long as the blowing rate increased, in consonance with the flow topology of the base flow (Fig. 5.13). At lower purge intensity cases, the mode appears concentrated at the exit of the purge channel and associated to the weak shear layers that occurred on the mixing of the base region and the purge flow. Subsequently, for larger blowing rates the mode changes its shape becoming more similar to the structures observed in common channel expansions[37, 91], where it adopts the shape of two enlarged lobes bounded by the strong shear layers of the upper and lower tips of the trailing edge .

The behaviour of the instability amplification rate is different at low and high blowing intensities. For a weak trailing edge blowing the rate of change of the amplification rate of the mode is high, and small variations of blowing rates would produce a rapid change of the global mode behaviour from stable to unstable; at higher blowing rates the tendency is smoother and the changes in stability behaviour are less abrupt. This fact can also explain the small differences between the RANS results and the stability analysis. Namely, according to RANS computations, the flow remains non-symmetric until a blowing rate of around $42 \%$, compared to a value of $38 \%$ for the stability analysis. However, it is normally observed that, when the amplification rate of the instability is not large enough, the flow can remain in a "unstable" situation for a long time, unless a perturbation is introduced in the flow triggering the new flow configuration[149].

\subsubsection{Sensitivity analysis}

The overlapping of direct and adjoint modes permits the calculation of the structural sensitivity, associated to the regions where structural modifications of the flow would have a major drift on the eigenvalue associated to the global mode. These regions, depicted in Figure 5.14 help to localize the instability core, here symmetric with 


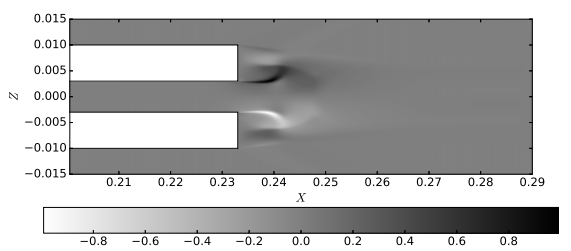

(a) $18 \%$ blowing rate

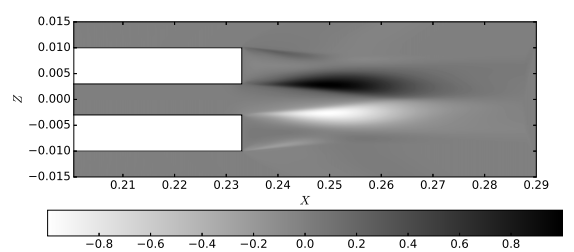

(c) $30 \%$ blowing rate

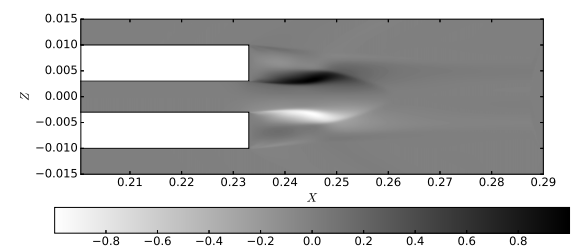

(b) $20 \%$ blowing rate

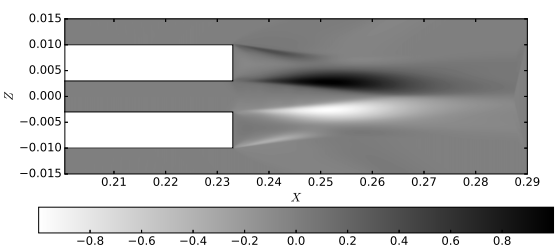

(d) $40 \%$ blowing rate

Figure 5.13: Evolution of the streamwise component of the eigenmode with the blowing rate.
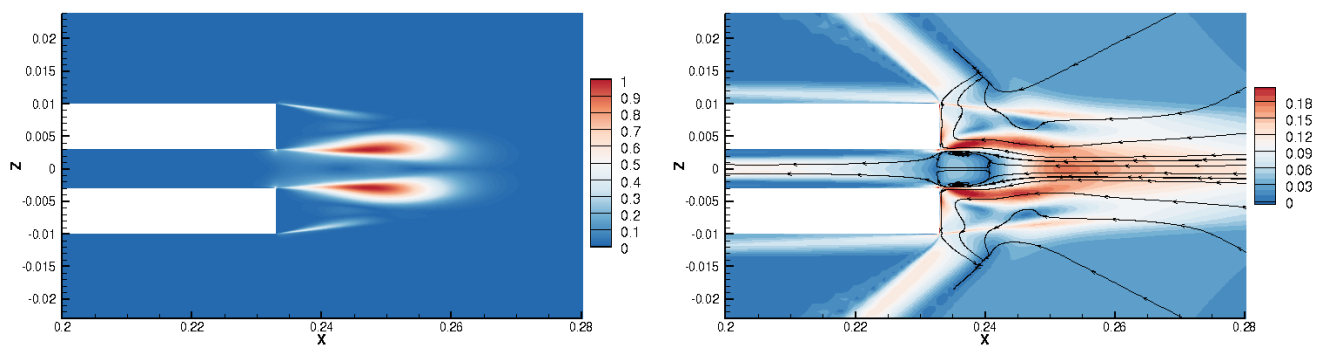

Figure 5.14: Sensitivity gradients of the leading eigenvalue. Structural sensitivity (left) and sensitivity to steady forcing (right), $\nabla_{\mathbf{q}_{\mathbf{f}}} \sigma$. here shown as the module of the momentum components.

respect to the mid plane and close to the end of the purge channel, coincident with the weak shear layers formed from the mixing of the cooling jet and the trailing edge recirculation areas. Small differences were found for changes in the blowing rate intensity. These results also corroborate the choice for the reduced domain limits, as the structural sensitivity is completely confined inside the bounds of the domain.

The sensitivity to changes in the base flow and to a steady force, normally represented by a complex vector field, are here constructed only by its real part due to the stationary nature of the unstable global mode, with null imaginary part. The information provided by the vector field indicates where an arbitrary modification $\left(\delta \overline{\mathbf{q}}\right.$ or $\left.\delta \mathbf{q}_{\mathbf{f}}\right)$ applied in the direction of the gradients will have a greater destabilization impact on the behaviour of the eigenmode. Figure 5.14 shows the sensitivity to steady forcing classic contour field, expressed as the module of the momentum 


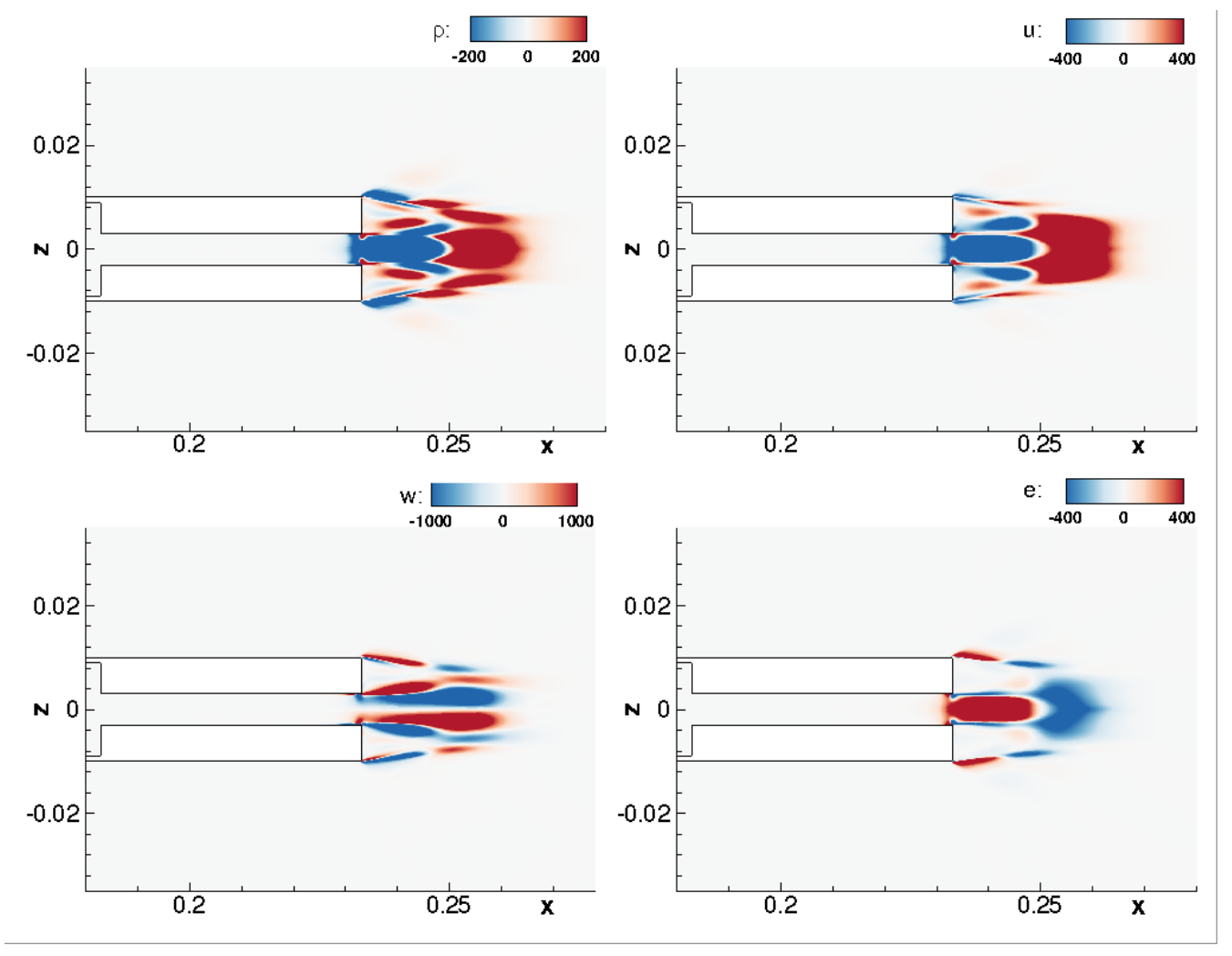

Figure 5.15: Breakdown by primitive variables of the sensitivity gradients of the leading eigenvalue to changes in the base flow.

components. Additional streamlines have been drawn to indicate the direction in which the application of a force will generate a positive drift on the real part of the eigenvalue. The global mode related with the pressure bifurcation seems to be more sensitive in the mixing region, and downstream the end of the channel (Fig. 5.14-right). Except on the small area limited by the shear layers, the presence of a steady force applied with a reverse direction to the discharging flow will have a destabilizing effect on the eigenvalue. Perturbations with a vertical component applied close to the trailing edge surface could have a stabilizing effect if they are directed from the trailing edge tip towards the discharging channel. To complement this information, a breakdown by components of the sensitivity fields is gathered in Figures 5.15 and 5.16. Both figures confirm the sensitivity of the global modes to perturbations inside the base region and the influence of changes in the trailing edge shear layers. The evaluation of the sensitivity to steady forcing reveals that changes inside the injection plenum may have an effect in the base region global instability. 

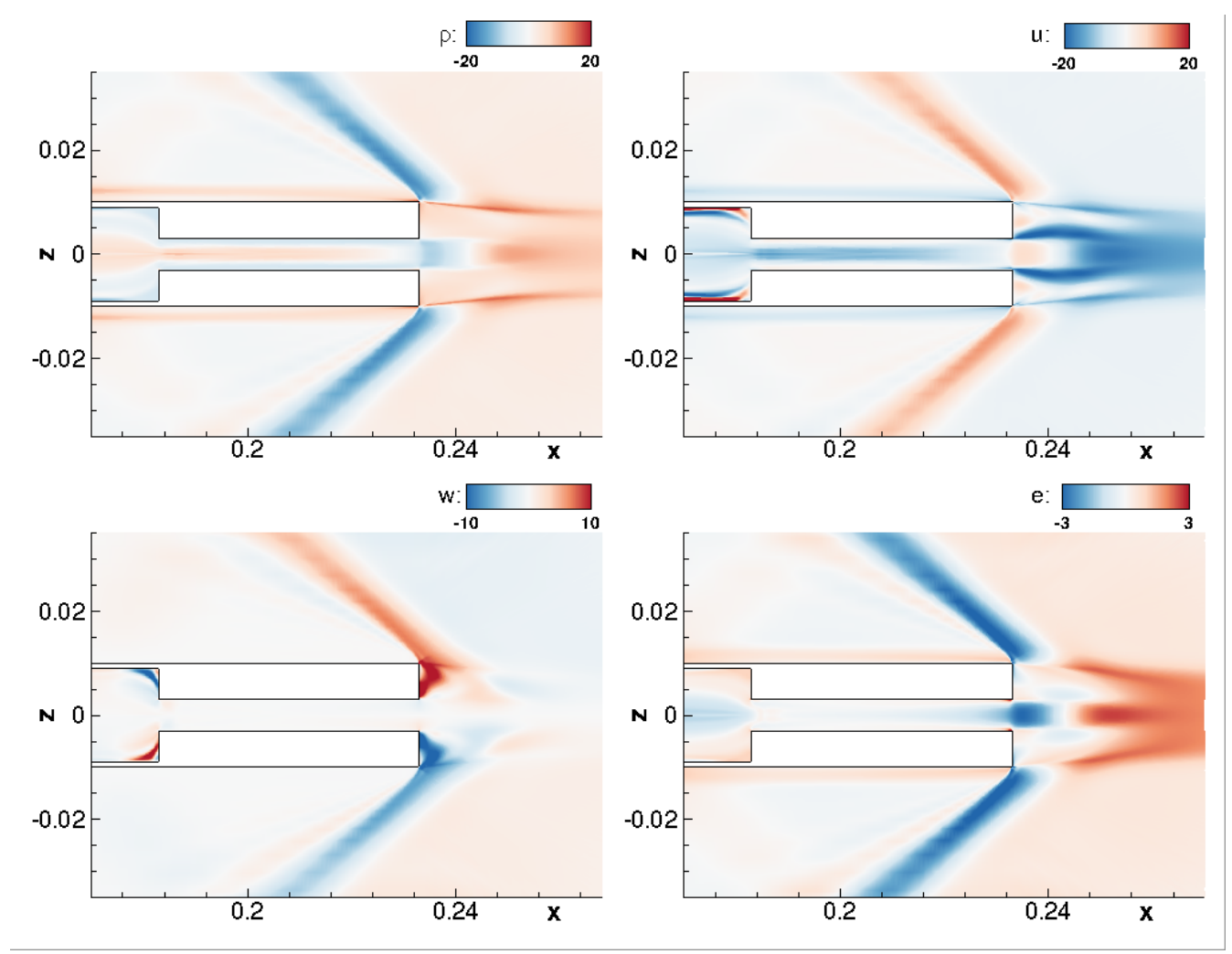

Figure 5.16: Breakdown by primitive variables of the sensitivity gradients of the leading eigenvalue to the application of a steady forcing.

\subsubsection{Conclusions}

The phenomenon of the sudden expansion related instability is replicated in this context for the supersonic blowing trailing edge problem, a simplified version of the flow configuration close to the trailing edge of a turbomachinery turbine blade. The presence of a bifurcation from symmetric to non-symmetric flow configuration as a function of the jet blowing rate had been observed but not explained. Previous analysis of this geometrical model suggested that the bifurcation was generated by a sudden expansion mechanism[122]. This fact was confirmed on this work with the use of Global Stability Theory.

The presented results link the non-symmetrical configuration with an unstable anti-symmetric eigenmode for a range of blowing rates, matching the start and end points of the pressure bifurcation with small error when compared with RANS results. Comparing the free stream to purge density ratio $\left(\rho_{\text {purge }} / \rho_{f}\right)$ of RANS solutions and stability analysis, an error value of $0.5 \%$ was found for low blowing rates, with an error of about 5\% for higher blowing rates. The eigenmode flow structures appeared to extend downstream in space as the purge intensity increased, revealing itself as a clear sudden expansion mode for higher blowing rates (having the same structure as those seen in Fani et al.[37]), but more concentrated at the 
end of the cooling channel for lower blowing rates. It is on this range of blowing rates where the mode takes an structure more opened to the lower and upper sides, in a shear layer shape, and where it is more sensitive to changes in the blowing rate.

When compared with a global mode related with a geometrical flow sudden expansion, its similarity in structure seemed clear despite the absence of viscous walls on the upper and lower sides aft the expansion that would bound the blow. In the configuration analysed here, however, the flow was confined due the the strong pressure gradients generated by the shear layers present on the trailing edge that limit the base region. This shear layers changed its shape with the blowing rate intensity, modifying this way the boundaries of the sudden expansion and thus affecting the eigenmode structure.

As observed, base pressure magnitude inversely correlated with trailing edge shock intensity. The variation of the cooling flow purge initially resulted in an increment of the base pressure and thus a decrease on the shock strength; for blowing rates higher than $25 \%$ this tendency reversed, the base pressure decayed resulting in stronger shock waves (Fig. 5.7). Due to the destabilizing effect of the pressure bifurcation, the region of maximum base pressure was affected by a variation on the pressure values of upper and lower sides at the trailing edge, which could furthermore abruptly change from one branch of the bifurcation to the contrary, generating strong loads on the turbine blades because of the change of the "polarity" of the pressure difference. Despite the apparently low difference between upper and lower pressure sides (only about a 3\%, as shown in Figure 5.6), the pressure bifurcation could had a strong effect on the surrounding flow topology and adjacent blades of the turbine. For blowing rates comprised between 18 and $38 \%$, the global instability forced the flow to deflect towards one of the shear layers (Fig. 5.12-(b)) generating a secondary shock wave upstream of the trailing edge shock in only one side (Fig. 5.10). This additional shock system, placed in only one side of the blade, would change to the opposite side when the deflected purge flow changed its direction due to a bifurcation branch change, therefore generating additional and non-symmetrical loads on the turbine cascade. If this intermittent non symmetric shock system is replicated all over the turbine blades, considering independent changes and interactions with upstream and downstream stages of the turbine, the consequences in the aerodynamic loads can be very important. In order to avoid these risks, one should increase the blowing rate up to an state of no variation (symmetry), implying an important increment on base pressure losses and intensity of the trailing edge shock wave system. The non time dependent nature of the instability would made it difficult to be controlled by frequency modulated systems (as those proposed by Saracoglu et al.[121]), but may be suitable for passive control or upstream flow modulation. The possibility of controlling the pressure bifurcation avoiding the mentioned problems would allow to exploit lower blowing rates for base region modulation, without undesired consequences.

The sensitivity analysis of the leading global mode indicated that the core of the instability is confined in the base region, without extending inside the cooling 
jet channel. The most sensitive areas appear to be the pair of mixing layers by the ejected flow, together with the trailing edge main shear layers. High sensitivity values near the wall suggest that flow actuation or surface modification in this area would have a great effect on the instability behaviour. Furthermore, it has been revealed that local actuation inside the injection chamber might have a downstream effect in the global instability, opening the door to enforce flow control by cooling flow modulation.

Understanding the mechanisms of the non-symmetric flow configurations present on a supersonic trailing edge, using a simplified model as the one described in this work, is the first step on preventing unwanted loads on turbine blades by Global Stability Analysis. The methodology here explained can be applied without changes to more realistic cases, where the pressure gradient between both sides of the aerofoil can play an important role on damping/exciting the sudden expansion mode. A closer approximation to the problem could allow to damp its sources (the unstable eigenmode) and even develop optimization techniques with direct feedback from the eigenvalue analysis.

\subsection{Further Investigation}

\subsubsection{Introduction}

This study aims to fully characterize the transient flow topology around a base bleed configuration on a trailing edge, and the benefits that flow injected at the base region can have on flow control and aerodynamic performance. In particular, the main goal is to take advantage and promote the observed Coanda effect, analyzing its capabilities and the physical mechanisms responsible of its generation. Despite of the abundance of literature on the study of flow bifurcation on a sudden expansion geometry $[4,13,38]$, and on the influence of base bleed effect on a turbine blade flow $[92,107,122,143]$, non of the references above mentioned covered the apparition of a non-symmetrical configuration on a base bleed configuration. One of the objectives of this research is therefore extend the available resources to identify this phenomenon and propose different ways to control and exploit it. To achieve this, a simplified body with a blunt trailing edge and a dedicated injection chamber for base bleed is considered, exploring a wide range of parameters to investigate their influence on the physical global modes responsible of the Coanda effect. The sensitivity to changes with the Mach number, the base bleed intensity, and the shape of the trailing edge tip are explored, identifying those configurations with optimal characteristics for base flow control.

Flow solutions are obtained solving the compressible Navier-Stokes equations using an unsteady RANS approach, whereas the stability and sensitivity analysis are be computed using in house numerical libraries for large-scale eigenvalue problems. Furthermore, using the information gathered in the numerical analysis, passive and active flow control most sensitive regions are identified and characterized. Although 
it is not contemplated in this paper, this study goes beyond the numerical approach, using the same geometrical body to experimentally investigate the apparition of the Coanda effect and correlate it with the numerical experiments. State-of-the-art flow visualization and measurement techniques are employed to analyze the flow around the body in a trisonic wind tunnel facility. The numerical campaign is therefore designed to be validated by experimental means.

The rest of this section is organized as follows. Section 5.2.2 summarizes the experimental setup and the description of the geometrical model, while in Section 5.2.3 the numerical procedures to obtain the base flow and stability solutions are reviewed. In Section 5.2.4 and 5.2.5 the main results of the flow characterization and flow control are described, finishing in Section 5.2.6 with the main conclusions of this study.

\subsubsection{Experimental methodology and model description}

Although no experimental results are provided in this paper, the experimental analysis is a crucial part of this research. For illustration, and for a better understanding of the numerical experiments design, the experimental set up is briefly described here.

The Purdue Experimental Turbine Aerothermal Lab (PETAL) facility has a linear wind tunnel conceived for low Technology Readiness Level (TRL 1-2) studies, which perfectly matches the requirements for the experiments to be carried in this research. It is a trisonic facility, covering from low subsonic to supersonic conditions, together with a wide envelope of Reynolds number conditions. This is accomplished through the independent control of inlet total pressure and temperature with a heat exchanger, as well as the control of the downstream pressure from atmospheric to vacuum conditions. The test section has a prismatic shape with dimensions 540x230x170 mm (length $\mathrm{x}$ width $\mathrm{x}$ height), and its unique visual access from the 4 fully transparent windows allow to combine different flow visualization techniques to characterize the aerothermal phenomena involved in the problem treated in this research. The PETAL facilities allow to use optical measurement techniques such as high frequency particle image velocimetry (PIV) and Schlieren flow visualization, that will be combined with pressure and heat flux measurements to provide an extended dataset of the experimental analysis. An extended description of the PETAL facilities can be consulted on [99].

The geometry of the model was therefore designed to be used in the trisonic range, with a blockage ratio with respect to the tunnel height lower than 8 to avoid excessive interference of the wind tunnel walls with the flow topology. The body consisted on a zero-camber airfoil of $160 \mathrm{~mm}$ chord $(c)$, with a fitness ratio of 8 , where a Haack Series[103] nose shape was chosen to adapt the flow without any detachments or very strong gradients for the three investigated flow regimes. The base bleed injection system was defined by a plenum area where the cooling flow is injected in subsonic conditions, and a straight channel into which the flow passes 


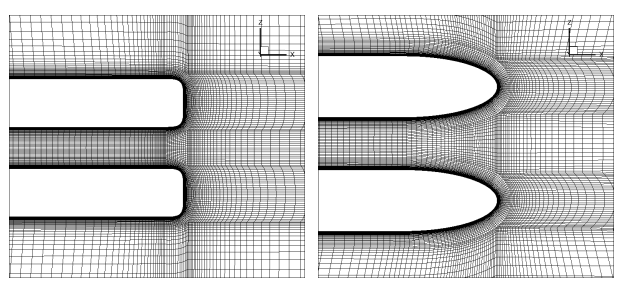

Figure 5.17: Comparison of trailing edge shapes used on the analysis. Left image shows the "straight" (super ellipse) shape, with the "rounded" (ellipse) shape on the right image.

through a contraction that is finally discharged at the base region. The aspect ratio of the contraction was kept to 3 , to ensure subsonic conditions at the plenum area, ending on a base bleed slot of $6 \mathrm{~mm}$ height. Two trailing edge tips were considered for the analysis, to study the variability of the Coanda effect with the geometry shape. An almost straight shape, defined by a supper-ellipse with an exponential parameter of 5, was used as a reference case, and will be referred as "straight" trailing edge along this paper. This geometry was similar to the one studied in previous works[79, 81, 122], where a mild-Coanda effect (where the flow was deflected by a pressure difference and will follow the shape of a recirculation area, but without been attached to a surface) was observed to appear at the base region, due to the first instability of the sudden expansion of the base bleed channel. The second trailing edge considered for analysis was designed with an ellipsoidal shape, where its curvature was expected to produce a full Coanda effect (with a flow deflection related to the attached flow following the curvature of just one of the surfaces). This shape will be referred as "rounded" along this paper. Both trailing geometries are shown on Figure 5.17.

\subsubsection{Numerical experiments}

To predict the behavior of the model and to perform a design of experiments, the geometry of the two-dimensional model and the wind tunnel walls were reproduced on a set of numerical simulations. To avoid non-physical boundaries on the computational domain, further inflow and outflow boundaries were imposed on those simulations were subsonic conditions were present.

Three different sonic regimes were investigated, with an optimized mesh topology for each configuration (Fig. 5.18). A Mach number at the inflow of 0.4 was taken to analyze the behavior of the cooling flow at subsonic conditions; an inflow Mach number of 0.7 , that produced a flow speed of $\mathrm{Mach}=1.1$ at the trailing edge of the model, was chosen to evaluate transonic conditions; and a third configuration with flow speed inflow of Mach=2 was used for supersonic flows analysis.

Grid spatial convergence was evaluated for a particular time step of the URANS simulations, with five different meshes (M0, M1, M2, M3, and M4, with each following mesh having double the points of the previous one) being used for the analysis. 

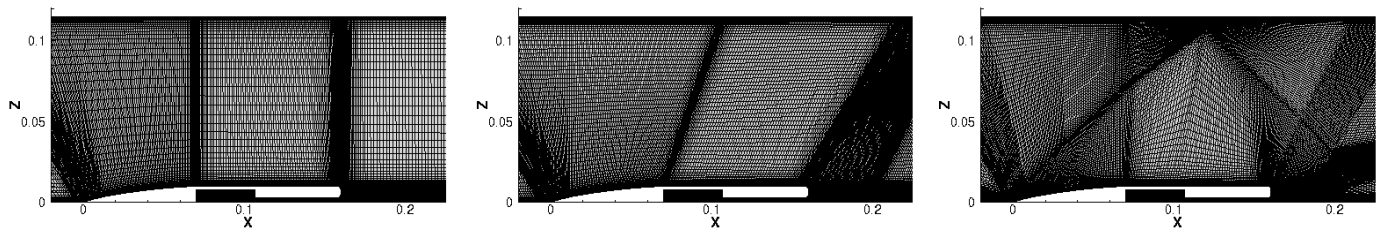

Figure 5.18: Mesh topology used for the different sonic regimes: Subsonic (left), transonic (centre), supersonic (right).
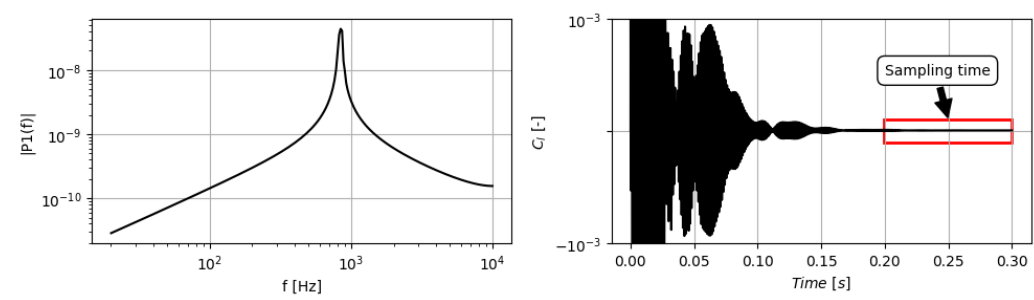

Figure 5.19: FFT analysis (left) of the lift coefficient sampling from an unsteady subsonic simulation (right).

The Grid Convergence Index (GCI)[158] was evaluated for three meshes, keeping the coarsest mesh (M0) as the stencil for the convergence analysis. The density values of each mesh were interpolated into M0, and the GCI was calculated using the Root Mean Square values. The results of the analysis are not included here for conciseness. Temporal discretization was done ensuring that each period of the dominant temporal fluctuations was covered with at least 40 timesteps. Due to this criteria, different time steps were used for each regime, varying from $10^{-5} \mathrm{~s}$ for subsonic conditions, to $10^{-6} \mathrm{~s}$ for supersonic flow conditions. The values of the monitored flow variables were extracted or averaged in a window of at least 100 periods, after the flow was already developed into a periodic state (Fig. 5.19).

On the subsonic and transonic meshes, total pressure and total temperature were imposed at the inlet, with an ambient pressure at the outlet boundary. On the mesh used for supersonic conditions, constant values were imposed at the inlet using a supersonic inflow boundary condition, with an exit-pressure condition at the outlet, were a differentiation of direct extrapolation or back pressure condition was done depending on the flow Mach number at the outlet cells. For all the meshes, adiabatic viscous walls were considered for the upper and lower walls, and for the blade model. Finally, a reservoir-pressure inflow was used to provide the cooling flow through the plenum area, imposing total pressure and temperature values. For the sake of readability, the coefficient $C_{b}=p_{0 \text { purge }} / p_{0 \infty}$, relating the total pressure of the reservoir pressure boundary condition with the total free stream pressure, was chosen to describe the pressure ratio of the base bleed flow.

The real non-symmetric operator A (Eq. 2.6) was extracted using the firstdiscretize-then-linearize technique implemented in the DLR TAU-code solver from 
either base or mean flows, and saved using a compressed sparse row format. The generalized eigenvalue problem was solved exploiting the capabilities of external libraries (as PETSc[10], MUMPS[5] or ARPACK[71]) to perform a complete LowerUpper (LU) decomposition of the input matrix and obtain the eigenvalues of interests, being these only a small range of the eigenvalues of the total spectra, using the Implicitly Restarted Arnoldi Method (IRAM) algorithm. Likewise, direct and adjoint eigenmodes of the system were extracted and processed afterwards to obtain the sensitivity maps.

Since the study aims to understand the apparition of the Coanda effect for different flow configurations, the nature and development of the non-symmetrical behavior of the injected flow needs to be analyzed from its onset, this implying the need of doing a global stability analysis around a symmetric base flow. To enforce this symmetry, only half of the domain was considered for the numerical simulations performed for stability analysis, imposing a symmetry boundary condition on the plane of symmetry $(x y)$. After obtaining a "half" domain flow solution, mesh and solution were mirrored into the symmetry plane and the stability analysis of the full domain was carried out. The global analysis of the mirrored-full domain around this symmetric flow at different flow conditions will show non-symmetric modes in the analysis, which could eventually grow and generate a new non-symmetric flow configuration.

\subsubsection{Flow characterization}

\subsubsection{Subsonic flow}

Subsonic analysis was designed for an inflow condition of Mach $=0.4, R e_{c}=9.5 \times 10^{5}$, total free stream temperature of $430 \mathrm{~K}$, and with a static temperature for the base bleed of $300 \mathrm{~K}$. The flow behind the body is characterized by the classic Von-Karman vortex street, with a characteristic Strouhal number of 0.264 . Vortices separate from the upper and lower sides in an alternate manner, producing an oscillating behavior of the wake and the base region (Fig. 5.20-left). The two shapes of the tested trailing edge have little influence on the flow topology when no cooling flow is applied at the base region, showing differences on the order of $0.1 \mathrm{KHz}$ on the frequency of the vortex shedding.

The dimensionless pressure relations for straight and rounded trailing edges when base bleed is applied can be seen in Figure 5.21, where the base pressure of upper and lower sides of the trailing edge are plotted against the base bleed intensity. The effects produced by the base bleed flow on a straight trailing edge can be divided in four different phases, denoted on the figure from phase I to phase IV. First, when cooling flow is ejected with low intensity at the base region, an initial filling effect is produced and the base pressure increases. After that, the bleed flow introduces a source of symmetry on the wake downstream, and the vortex shedding is actually neutralized for a certain range of blowing rates. It is at these rates when the first 

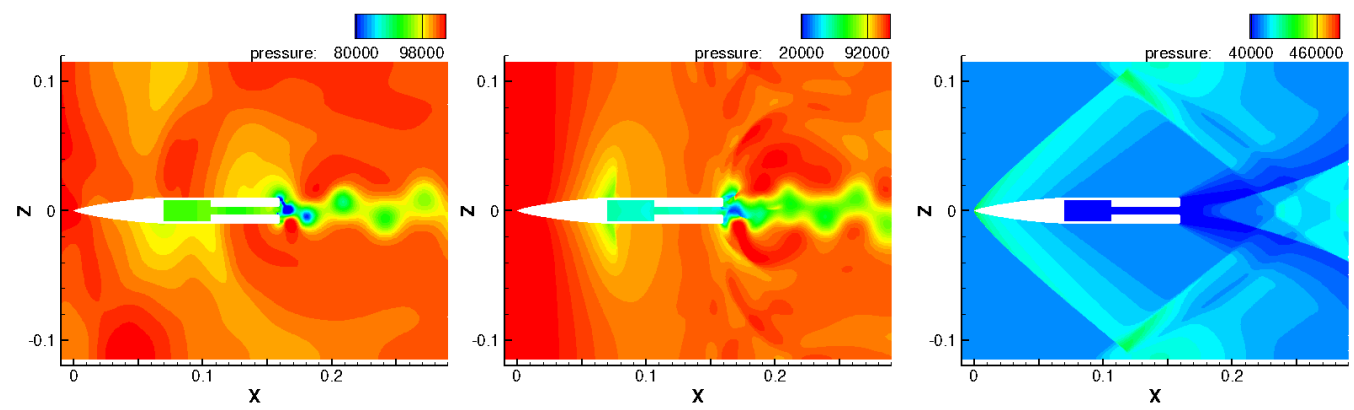

Figure 5.20: Temporal snapshot of static pressure contours for subsonic (left), transonic (center), and supersonic (right) regimes. Base bleed not active.

presence of the non-symmetrical flow can be detected, as the first instability of the sudden expansion appears. Named as phase II, is at this stage when the base bleed flow is deflected towards either the upper or lower side of the trailing edge in a mild Coanda effect style, as the flow is attached to the recirculation areas at the base region. However, when the pressure ratio $C_{b}>0.95$, the presence of the vortex shedding reappears with a frequency that is twice the one present with no blowing. This increment in frequency has been previosly related in the literature[92], and the authors believe that this happens due to the onset of the secondary instability of the geometrical sudden expansion of the base bleed jet channel. Base pressure decays at these blowing rates, reaching values similar to those without blowing, and with constant asymmetries on the pressure averaged values of upper and lower sides, showing an change in direction of the ejected flow that is not periodic, but strongly affected by the secondary vortices separated from the injection channel. Finally, at phase IV, for base bleed values with $C_{b}>1.4$ the flow inside the injection channel becomes supersonic and the momentum of the ejected flow keeps the flow symmetric, weakening any oscillation and increasing the base pressure towards a final plateau of $p_{b} / p_{\infty} \approx 0.94$.

If the rounded trailing edge geometry is considered, the effects of the base bleed are milder, as the geometrical sudden expansion is not present. First, an increment on the base pressure is observed as the base region is filled with cooler flow. For values of $C_{b}$ as low as 0.87 , the non-symmetrical flow configuration of phase II already appears, as a full Coanda effect can be observed at the end of the injection channel. However, the effect quickly disappears, and for base bleed intensities higher than $C_{b} \approx 0.92$ the base pressure remains constant, without any vortex presence or flow oscillation downstream the trailing edge. Phase III, related with reapparition of the shedding with higher frequency, is not present in this case.

Whereas the non-symmetrical configurations with a straight trailing edge are related with a difference in pressure between the upper and lower sides, as the mild-Coanda effect takes place at the base region when one of the recirculation areas (upper/lower) grows at the expense of the contrary, the apparition of a nonsymmetrical configuration on a rounded trailing edge is not linked with a pressure 

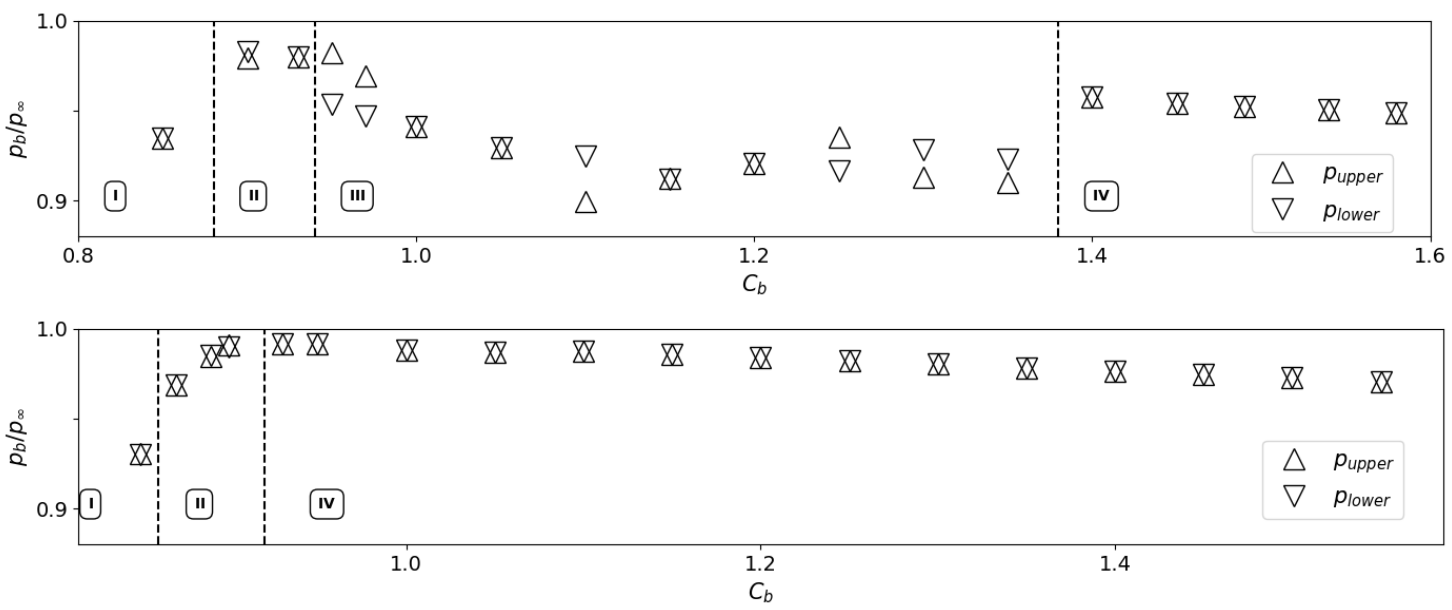

Figure 5.21: Base pressure evolution with the base bleed intensity in subsonic regime. Results for straight (upper) and rounded (lower) trailing edge geometries.
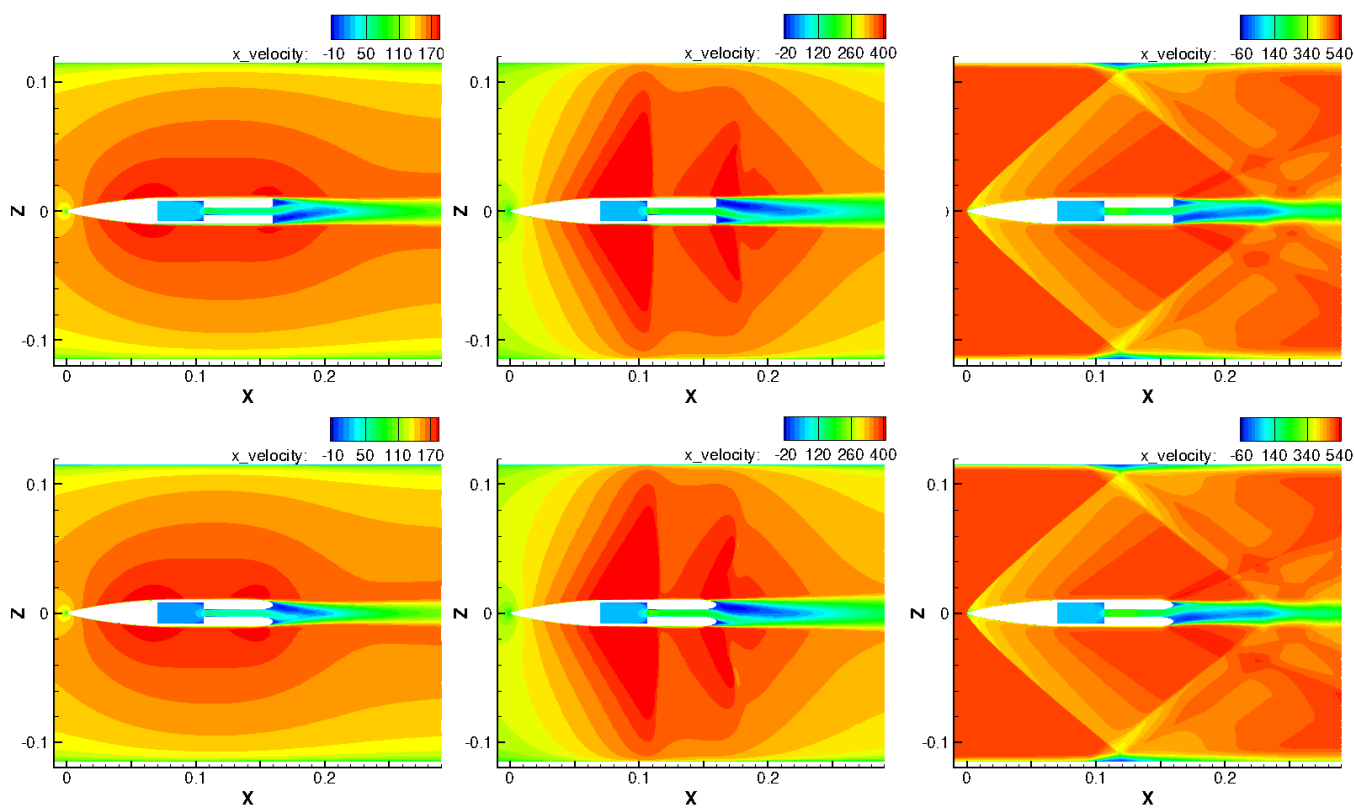

Figure 5.22: Temporal snapthot of streamwise velocity contours for base bleed nonsymmetrical flow configurations (phase II), caused by the global instability of the sudden expansion. Upper row shows straight trailing edge flow solutions (subsonic to supersonic, from left to right), and bottom row shows the rounded trailing edge flow solutions.

bifurcation. The identification of this phenomenon is therefore linked to flow visualization or velocity components monitoring. Velocity contours are shown on Figure 5.22 , for a better illustration. 

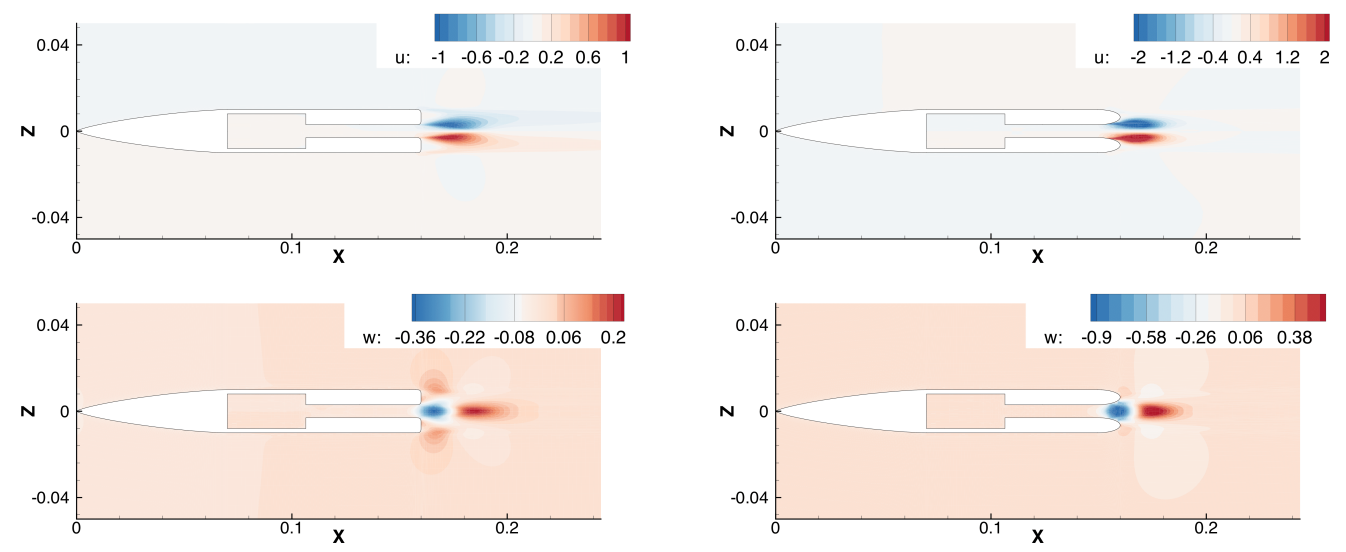

Figure 5.23: Global mode related with the geometrical sudden expansion, subsonic regime. Streamwise $(u)$ and vertical $(w)$ velocity perturbations are shown for both trailing edge configurations.

The stability analysis of configurations from phase II (non-symmetrical flow condition) allows to recover the global mode related with the sudden expansion of the channel, for both trailing edge geometries. For only few configurations a converged steady RANS solution (base region approach) could be obtained. As the sources of unsteadiness in the flow are multiple, a mean flow analysis was therefore performed. 300 periods of oscillations from a URANS simulation over half domain (cropped in the $x y$ plane) were averaged to extract the mean flow. Streamwise and vertical velocity perturbations contours of the global mode related with the instability are shown in Figure 5.23. The shape of the eigenmode is similar to the identified in a sudden expansion phenomena, with the main non-symmetrical streamwise perturbations located at the base region in the form of two anti-symmetrical lobes. This global mode becomes unstable for a certain range of blowing rates and drives the change of direction of the ejected flow[79]. The eigenvalue associated with the sudden expansion global mode has no associated frequency, despite the pressence of oscillations in the flow, these related with the vortex shedding. The instability is stable in time, and the flow will remain attached the side it initially contacted, even if an oscillating vortex shedding co-exists downstream.

\subsubsection{Transonic flow}

Inflow total temperature was kept constant at 430K, with a Reynolds number of approximately $R e_{c}=1.8 \times 10^{6}$, and a Mach number of 0.7 . The static temperature for the base bleed was again set constant to $300 \mathrm{~K}$. The flow accelerates over the model, reaching the trailing edge at a speed of Mach=1.1. Two symmetric shock waves develop at the inflection point of the geometry, at the end of the nose ogive, which position will remain almost constant, slightly varying for each base bleed intensity. The blockage effect of the tunnel walls produces an "unstart" effect on 

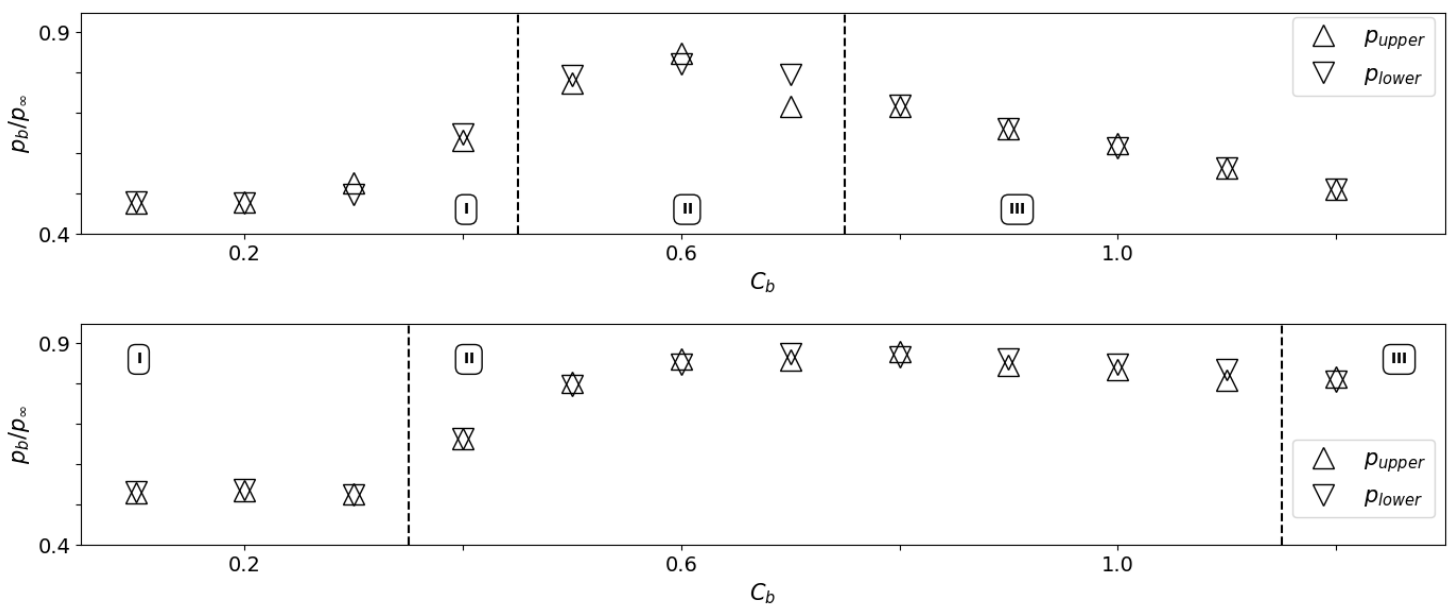

Figure 5.24: Base pressure evolution with the base bleed intensity in transonic regime. Results for straight (upper) and rounded (lower) trailing edge geometries.

the downstream shock waves, as the oscillation of the wake behind the body limits the mass flow that passes through the trailing edge section (Fig. 5.20-center). The trailing edge shock waves appear downstream the body and travel downstream with the separated vortices in an alternate manner with an associated Strouhal number of 0.324 .

Interestingly, for this regime the shape of the trailing edge shows to have a major impact on the base region flow behavior (Fig. 5.24). If the straight trailing edge tip is used, the application of base bleed on this configuration produces again an initial increase in base pressure (phase I), followed by an apparition of a non-symmetric condition for those blowing rates related with maximum base pressure (phase II). This stage is linked with a strong reduction in the vortex shedding frequency, which eventually increases again with the final decay in base pressure as the base bleed flow speeds reach supersonic regime (phase III). However, a rounded trailing edge shape shows a delay on the later decay in pressure, at the same time that the Coanda effect prevails over the symmetric condition for larger blowing rates.

The stability analysis to recover the global mode related with the Pitchfork bifurcation of these configurations could however not be completed with the current methodology. Due to the nature of the flow topology, the solutions of a simulation of a mirrored half domain (to ensure symmetric conditions) will not match the complementary flow solution of a full domain simulation, an essential requirement for the methodology here presented. Other methodologies (continuation methods, for example) will be applied in the future to tackle this problem.

\subsubsection{Supersonic flow}

Due to the isentropic expansion of the flow that occurs through the convergentdivergent geometry that would be installed at the wind tunnel to analyze supersonic 
conditions, the total temperature at the inflow boundary considered for the supersonic flow simulations was decreased to $300 \mathrm{~K}$. To still provide cooler flow at the base bleed, the temperature of the injected flow was corrected to $120 \mathrm{~K}$. Inflow speed was set at $\mathrm{Mach}=2.0$ at the boundary. The flow around the body is purely supersonic, except for the base region area where a subsonic recirculation section takes place, confined by the strong shear layers of upper and lower sides of the trailing edge. Two oblique shock waves depart from the sharp leading edge, being reflected at the upper and lower walls and impacting downstream the base region with the trailing edge shock system (Fig. 5.20-right). This flow system has been carefully studied in the past $[79,122]$, so it is briefly described here. As the boundary layers of upper and lower sides approach the trailing edge, they separate forming two symmetric shear layers that confine a subsonic recirculation area, the base region. The main flow first expands and accelerates through a Prandtl-Meyer expansion fan, just to later compress and adapt through a strong trailing edge shock wave. When no base bleed is applied, and there is no pressure difference between the upper and lower sides of the trailing edge, the flow downstream remains symmetric and steady, with no vortex shedding present.

The effects of base bleed on supersonic flow were described in detail by Saracoglu et al.[122] for an inflow Mach number of 1.5 and a straight trailing edge, and few changes from what was described in that scenario were found here. We again divide the flow topology changes in four phases, as the blowing rate increases. Low intensities are gathered in phase I, where base pressure increases keeping the symmetry of the flow. At a bleed intensity of $C_{b}=0.1$, a pressure difference appears between upper and lower sides, indicating the appearance of the sudden expansion first bifurcation. We marked this stage as phase II, that lasts up to $C_{b}=0.16$ and covers the maximum values of base pressure that are produced by the flow injection. Higher mass flows produce a decrement on the base pressure as the wake is pushed downstream by the jet and the base region flow is entrained into the base bleed flow. For values of $C_{b}$ higher than 0.20 , strong frequency fluctuations of the order of $S t=0.388$ appears, showing a non-symmetrical configuration with strong wake oscillations for $C_{b}=0.25$. This is linked with an increase in base pressure, that again drops when the symmetric state is recovered. Finally, phase IV arrives for $C_{b}$ values of 0.28 and higher, where the intensity of the base bleed flow keeps the flow symmetric with base pressure values similar to those without base bleed.

When a rounded trailing edge is considered, the Coanda effect appears early, as the flow is rapidly attached to one of the sides of the base bleed channel. A jet flow deflection can be observed for values of $C_{b}$ between 0.08 and 0.22 , with a maximum base pressure for a mass flow of $C_{b}=0.14$. At higher blowing rates it starts to decay. The strength of the jet eventually recovers the symmetric state, but without the appearance of high frequency oscillations, as it was shown for the straight trailing edge. Flow solutions for non-symmetrical configurations are shown on Figure 5.22. 

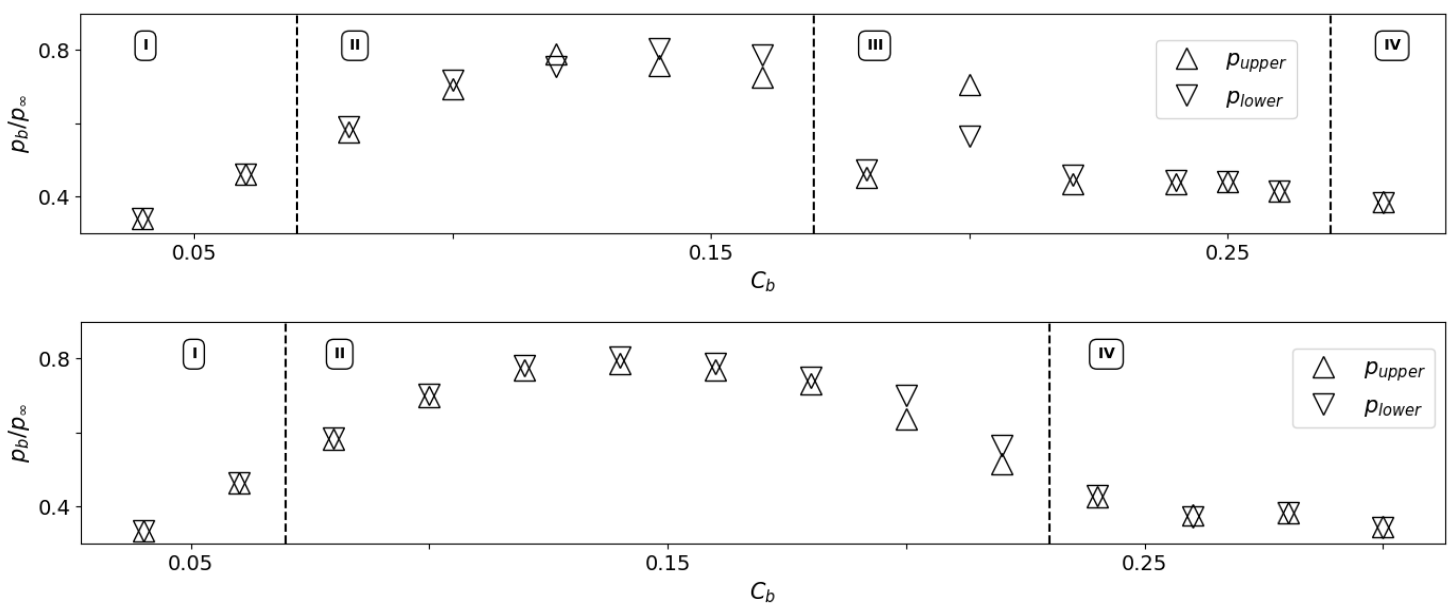

Figure 5.25: Base pressure evolution with the base bleed intensity in supersonic regime. Results for straight (upper) and rounded (lower) trailing edge geometries.

The flow topology, with few sources of unsteadiness, allows the calculation of RANS solutions for the half domain simulations, obtaining steady base flows for the stability analysis. The analysis was performed for chosen mass flows, within the blowing rates related with the non-symmetric configuration, revealing again the presence of the expansion mode, in the form of a Pitchfork bifurcation. However, the shape of the global mode is different from what could be observed for the subsonic case, as the perturbations will affect the shear layers and the trailing edge shock waves (Fig. 5.26). For this regime, it is also clear the influence of the trailing edge geometry on the shape of the eigenmode, that for a rounded shape it abandons the classic perturbation distribution of sudden expansion geometries of anti-symmetrical lobes to a more stylized and line shaped distribution.

\subsubsection{Base region flow sensitivity}

Controlling the flow downstream a turbine blade is one of the major challenges nowadays, as secondary flows drive most of the losses in this area. With the information extracted from this numerical analysis, we aim to identify those regions that would be more appropriate for flow control applications, reducing the time on a real design process.

For this we propose two ways of action, looking forward to passive and active flow control. The first is based on the modification or addition of geometry to the existent one in order to mitigate the instability, in this case to force a symmetric base bleed flow regardless the intensity of the jet. The second is however more complex, as we will be looking at the control of the flow at the base region by direct actuation on it. As the non-symmetrical phenomena has been linked to an unstable 

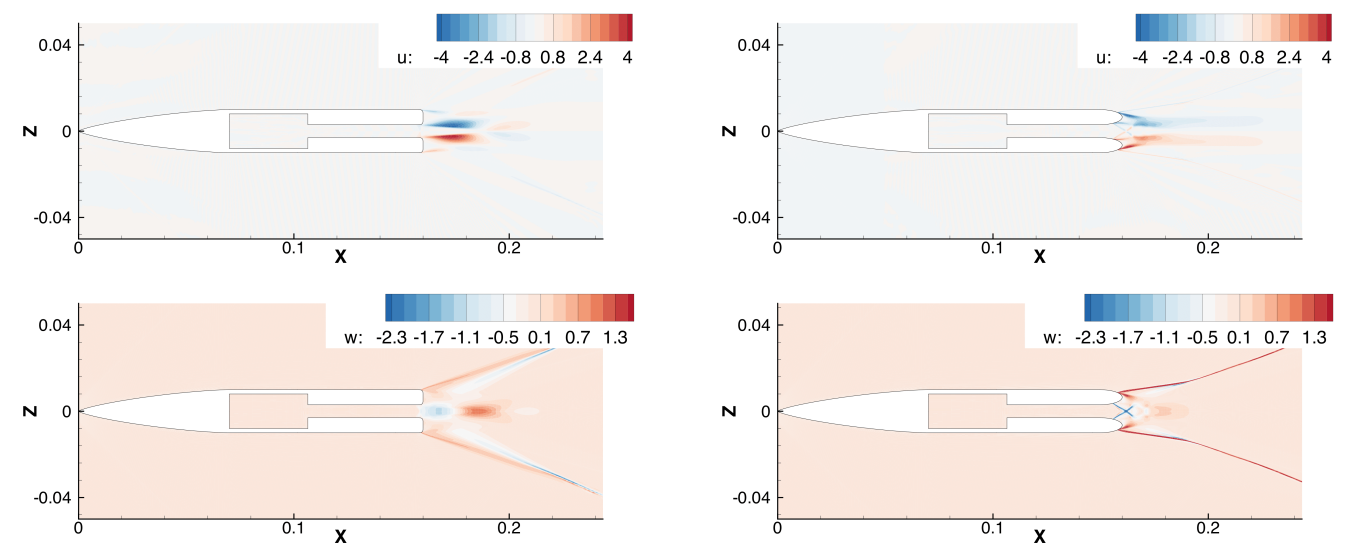

Figure 5.26: Global mode related with the geometrical sudden expansion, supersonic regime. Streamwise $(u)$ and vertical $(w)$ velocity perturbations are shown for both trailing edge configurations.

global mode, the sensitivity analysis of the related eigenvalue will provide valuable information on this topic.

The wavemaker regions (core of the instability) are first calculated as the structural sensitivity maps (contours shown on Figure 5.27), showing a strong difference between the straight and the rounded trailing edge shape. For the latest, the core of the instability is always close to the wall, near the flow detachment point in the inner side of the ellipse, with a very different distribution for the supersonic regime where a secondary region appears at the foot of the Prantdtl-Meyer expansion fan. The straight trailing edge presents however the core of the instability downstream the expansion, for both subsonic and supersonic cases, with a secondary sensitive area at the end of the injection channel wall. The structural sensitivity follows the shape of the global modes, being the main core of the instability mainly located at the base region, outside the injection channel.

As the global modes were obtained for a forced-symmetrical configuration, the sensitivity fields appear symmetric respect to the mid-plane, and they are effective regardless the direction of deflection of the flow. For all the cases, the core of the instability is contained within the recirculation area of the base region. This information is valuable not only to understand where are located the principal mechanisms of the instability, but to consider future stability analysis where a more expensive computational domain is required. Giannetti and Luchini[46] and Sanvido et al.[120] showed that to ensure a good accuracy on the calculation of the eigenvalues, the computational domain for the stability analysis should confine the complete instability core.

One of the most interesting results that can be extracted from the sensitivity analysis, are the vector field maps related to the sensitivity of the global mode (and its related eigenvalue) to the application of a localized steady forcing. Due to the 

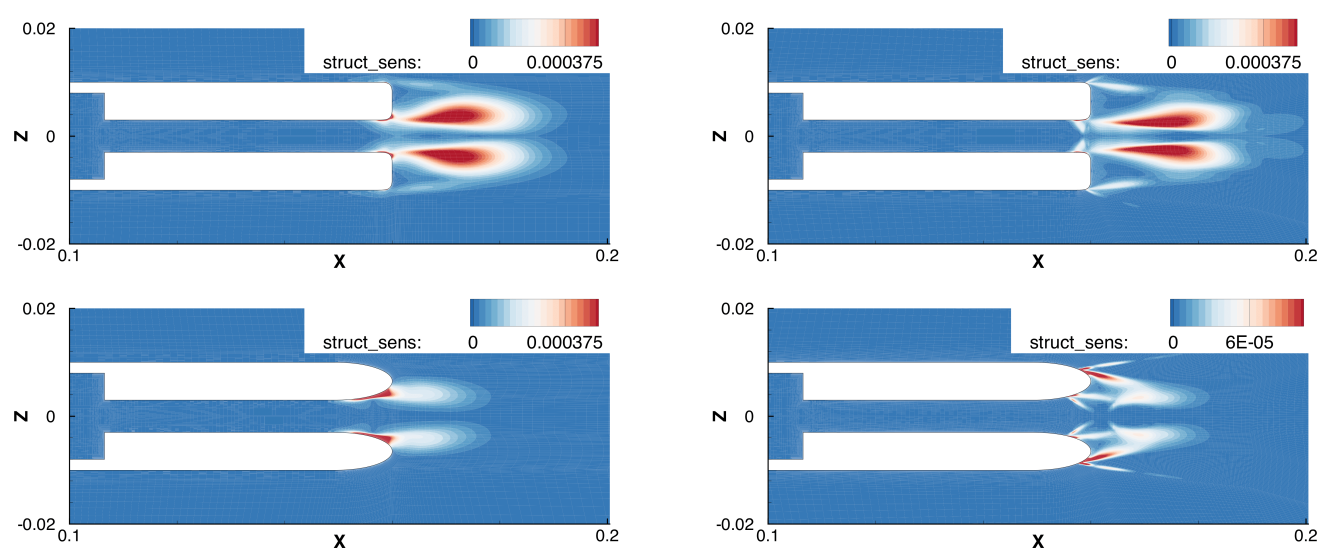

Figure 5.27: Structural sensitivity of the global mode responsible of the nonsymmetrical flow configurations. Left two figures (upper and lower) correspond to subsonic conditions, with the right two figures obtained for supersonic flow.

nature of the instability (with no associated non-frequency), this information could be very valuable in terms of active flow control. Both maps for the subsonic and supersonic configurations are plotted on Fig. 5.28, with the depicted streamlines indicating the direction in which a steady force would have a destabilizing effect on the global mode. For a straight trailing edge the most sensitive areas remain inside the cooling flow injection channel, very close to the walls and located near the exit. The subsonic regime appears to have a secondary area of sensitivity at the end of the trailing edge, where the main shear layers of the base region are formed. For a rounded trailing edge, however, the most sensitive regions to steady forcing are more elongated, with its core located on the area behind the separation point on the opposite side where the Coanda effect is taking place. In supersonic regime, an additional region in the center of the channel appears, related with the expansion of the injected flow.

As shown in Section 4.2.1, it is possible to calculate the sensitivity of the eigenvalue to the addition of a small control body to the flow field to control the instability. This is done by assuming a steady force $\mathbf{f}_{x z}$ in the direction of the flow, namely the drag force of a small cylinder with diameter $d^{*}$. If this analysis is performed near the bifurcation point, without loose of generality one can say that the sensitivity of the eigenvalue to the placement of a control cylinder at the coordinates $(x, z)$ would be[77]:

$$
\nabla_{\mathbf{f}_{x z}} \sigma=<\nabla_{\mathbf{q}_{\mathbf{f}}} \sigma, \mathbf{f}_{x z}>
$$

with $\mathbf{f}_{x z}=-\alpha \overline{\mathbf{q}}(x, z)\|\overline{\mathbf{q}}(x, z)\| / \Omega_{x z}$. Coefficient $\alpha$, constrained as $0<\alpha \ll 1$, normally takes the form $\alpha=\frac{1}{2} d^{*} C_{D}\left(R e_{d^{*}}\right)$. If this analysis is conducted for the global modes responsible of the change of direction of the flow, the results are similar to those obtained by Fani et al.[37], where it was shown that a small cylinder 

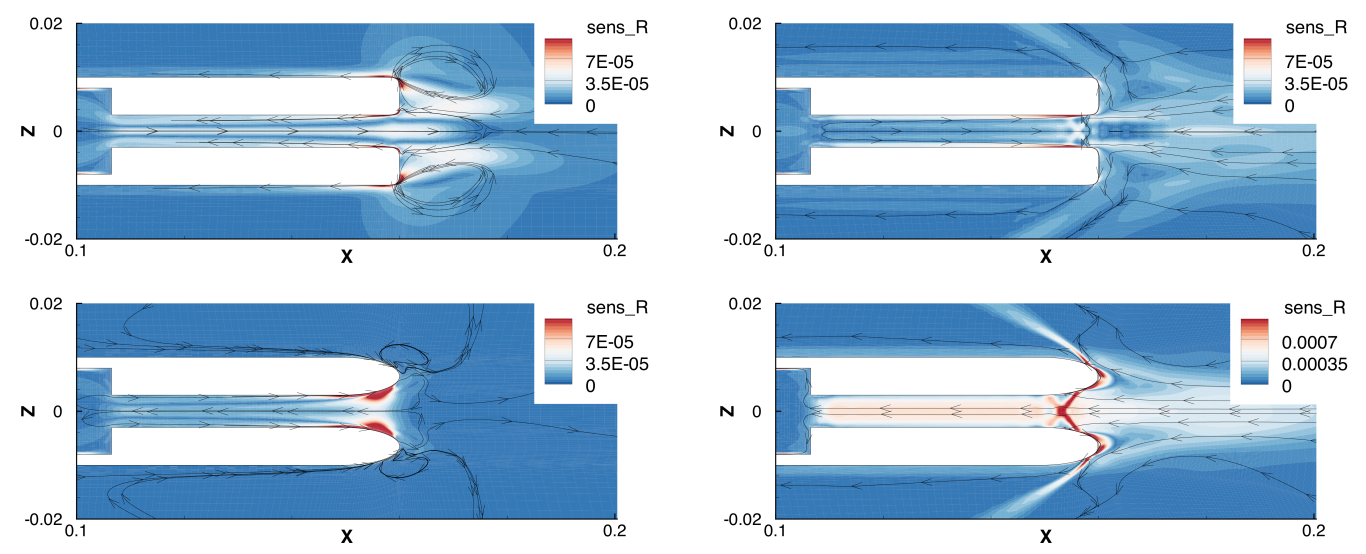

Figure 5.28: Sensitivity to the application of a steady force. Perturbations in the direction of the streamlines would have a destabilizing effect on the eigenvalue. Left two figures (upper and lower) correspond to subsonic conditions, with the right two figures obtained for supersonic flow.

placed at the "sweet spot" at the end of the channel before a geometrical sudden expansion can mitigate or neutralize the apparition of the instability. Sensitivity maps are shown on Fig. 5.29, where both flow regimes and trailing edge shapes are represented. Interestingly, the rounded trailing edge seems to be far more sensitive to passive control than its reciprocal configuration. For subsonic conditions, the presence of an additional body (or modification of the surface to generate additional drag) at the end of the base bleed channel will have a stabilizing effect on the eigenvalue. Inside a supersonic flow, this area extends upstream the channel, with two additional lobes downstream where a control cylinder could be placed. The analysis for passive control on the straight configurations, show that only specific locations will be suitable for the suppression of the instability by adding a control body.

\subsubsection{Conclusions and way forward}

The non-symmetrical flow configurations shown here can appear at the base region of a base bleed trailing edge, and are related to a fundamental phenomenon that is normally ignored on the big picture of turbine blade design. This phenomenon has an effect on the development and behaviour of secondary flows, and with the correct information could be exploited for better performance, or cancelled to avoid any undesired interferences.

With the aim of extending the available information in the literature regarding base region flow modulation by trailing edge blowing using coolant flow, a range of subsonic, transonic and supersonic cases have been analyzed. A combination of unsteady RANS simulations and stability analysis has been used to identify those 

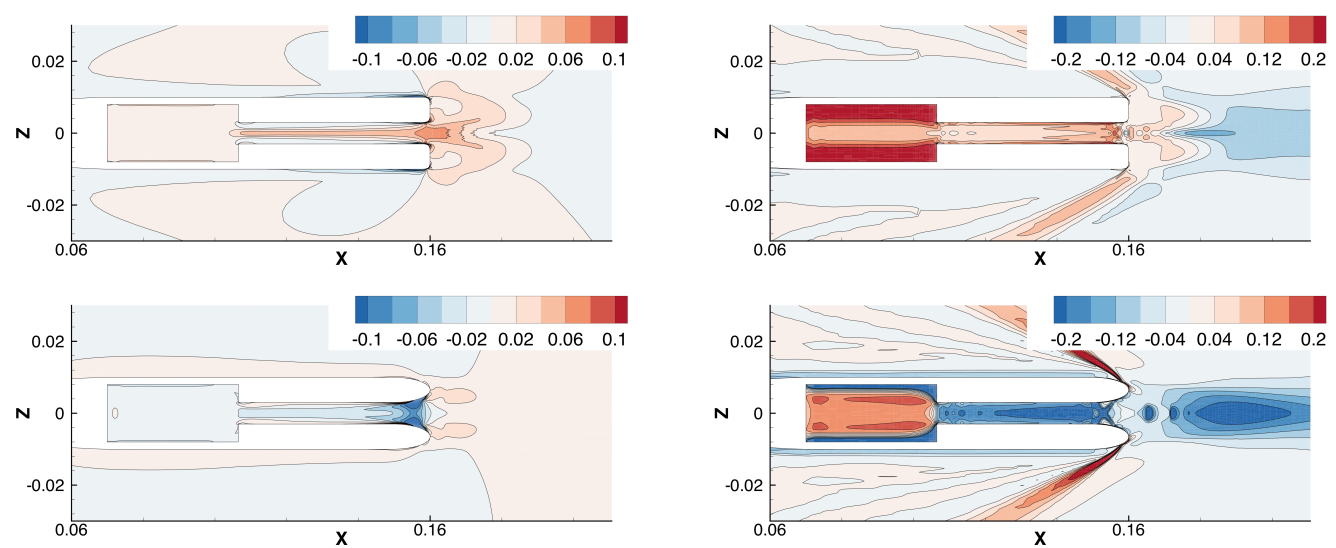

Figure 5.29: Localization of regions where the location of a small control cylinder will generate a major drift on the eigenvalue. Left two figures (upper and lower) correspond to subsonic conditions, with the right two figures obtained for supersonic flow.

blowing rate intensities for which base pressure presents a maximum value, and also which of these values are related with the apparition of a mild or full Coanda effect at the cooling injection channel. The global stability analysis revealed the presence of a global mode connected to the geometrical sudden expansion, that eventually drives and control the direction of the base bleed jet flow. It has been shown that a rounded trailing edge tip provides a less abrupt behavior on the base region pressure changes, accompanied by a higher flow deflection when the first instability of the expansion geometry becomes unstable. The use of rounded edges, moreover, seemed to neutralize the decay in base pressure that was observed for subsonic and transonic flows, as well as the related high frequency vortices that were identified for subsonic and supersonic regimes for the studied configurations.

The information extracted on the study, combined with the results of the sensitivity analysis based on the unstable global modes, will open the door to active and passive flow control techniques to modulate or neutralize the global instability present at the cooling channel, that is directly related with the apparition of a Coanda effect at this region. The addition of a small control body at the calculated "sweet spot" will keep the flow symmetric for a large range of base bleed flow intensities. 



\section{Part IV}

\section{Eigenvalue Sensitivity to Geometrical Changes}





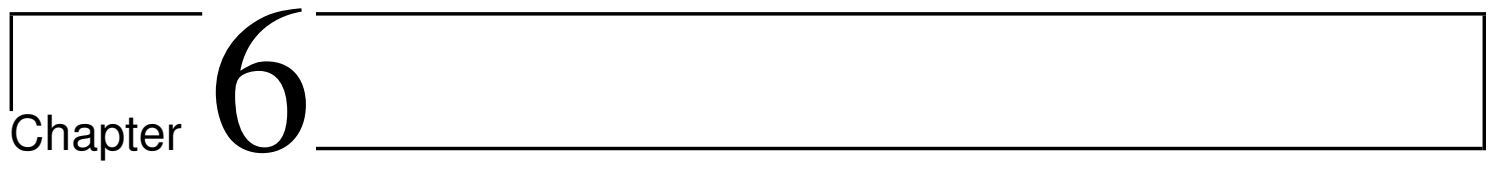

\section{Stability Analysis Based Geometry Optimization}

\section{Contents}

6.1 Introduction . . . . . . . . . . . . . . 94

6.2 Sensitivity of the eigenvalue to geometry changes . . . 96

6.3 Implementation and validation . . . . . . . . 99

6.3 .1 Analytic test case . . . . . . . . . . . . . . . 101

6.4 Numerical experiment. The cylinder wake instability . . 102

6.5 Optimization process ............... . 104

6.6 Control of the growth rate of the instability . . . . . 106

6.7 Control of the vortex shedding associated frequency $\ldots 107$

6.8 Discussion on the influence of the deformation control parameter, $\varepsilon \ldots \ldots \ldots \ldots \ldots \ldots$

6.9 Analysis and discussion . . . . . . . . . . . 108

6.10 Conclusions ................... 110

The sensitivity of a selected eigenvalue (normally the least stable, related with an unstable global mode) to changes in the surface geometry of the body of study is applied here to the first instability of the cylinder wake. The sensitivity gradients are used to guide an optimization process to control the instability: suppressing or enhancing it, and varying its associated frequency. This chapter is part of a submitted publication to the Journal of Computational Physics, under the title Eigenvalue Sensitivity to Surface Geometry Modifications. 


\subsection{Introduction}

On the pursue of better aerodynamic performance, optimization algorithms are the last and most time consuming steps of the design process. Typically, the targeted functional is directly related to the aerodynamic performance of the body (i.e. lift, drag or moment coefficients)[73] or to other flow parameters of interest (pressure drop[31], associated frequency[78], shear stress[2], etc.). Shape optimization problems have enabled great achievements with the development of optimal control and optimization algorithms based on adjoint methods, sensitivity and gradient calculations[104], and gradient-free methodologies[159]. For a detailed review, the interested reader is referred to the recent contribution of Skinner and ZareBehtash[134].

However, optimization does not have to be associated only to specific (aerodynamic) targets. Nowadays, in the search of more stable flow configurations, links between flow control, stability analysis and optimization loops are emerging, providing new and more efficient methodologies to address the underlying physics of the problem. Recent studies have pointed towards the optimization and control of flow configurations using global stability analysis as a source for the gradient calculations. Stability analysis studies how small perturbations that appear on the flow (naturally or by external agents) may evolve, either weakening or growing and becoming dominant as time evolves, depending on the characteristics of the dynamical system. From a mathematical point of view, stability analysis demands the resolution of an eigenvalue problem, whose eigenvalues determine the temporal evolution of the spatial perturbations, the associated eigenvectors (or modes). The combination of the obtained eigenpairs with those of the related adjoint eigenvalue problem provides relevant information of the response of the system to external modifications or variations of the intrinsic flow parameters or initial conditions[126]. Knowing the influence to variations of additional parameters, such as the turbulent viscosity, Mach or Reynolds number, or the angle of incidence of the incoming flow, would be an asset on understanding the phenomena. On this regard, much efforts are being addressed to obtain the regions of the flow where the global modes are most sensitive, namely, where small modifications of the flow could provide the maximum variations on the mode behavior. Of particular importance is the sensitivity to surface modifications, which may be efficiently considered in the optimization loop through local deformations of the nodes defining the surface, or using control parameters for the surface shape.

Along these lines, Gianetti and Luchini[46] revisited the concept of the 'wavemaker' area in the benchmark problem of the flow around a circular cylinder using global stability analysis. The authors identified the structural sensitivity regions, the first step to predict where passive control could be more effective on the delay of the onset of the vortex shedding on the cylinder wake. This phenomenon is widely known to be dominated by a global (absolute) instability. Gianetti and Luchini analytically obtained the optimal areas for the positioning of a small control device, 
matching the experimental results of Strykowsky and Sreenisavan[138]. Latter on, Marquet et al.[77] revisited the same flow problem and developed the sensitivity to baseflow modifications and steady forcing from a continuous approach, that was later replicated by Mettot[89] and Browne[20] for the discrete approach. Additional examples can be found in the work of Meliga et al.[87], who used the sensitivity and adjoint analysis on the study of axisymmetric bodies, optimizing the position of a heated ring behind an axisymmetric body on the control of the wake. The same authors studied the sensitivities to boundary forcing on the wake of disks and spheres[85]. Ferrer et al.[39] evaluated the influence of a control cylinder on the wake of an actuator disk, representing a simplified wind turbine, showing relevant changes on the behavior of the unstable mode. More recently, Tammissola et al.[140, 141] used a second-order perturbation analysis on the optimization of spanwise shaping and spanwise actuation of a cylindrical body to passively stabilize the vortex shedding. On a planar X-junction, Lashgari et al.[69] optimized the distribution of wall suction/blowing for the first flow bifurcation, giving also physical explanation to the second bifurcation using the results of the sensitivity analysis. As an example of eigenvalue-based and gradient-free optimization problem, Wang et al.[153] stabilized the global instability present on channel contraction using a genetic-algorithm optimization approach combined with structural sensitivity analysis, increasing the critical Reynolds number for the outbreak of the flow asymmetries by a 750 percent. This list probably omits many relevant works on flow control based on global stability, so the reader is encouraged to follow the reviews of Luchini[75] and Sipp[133] on adjoint analysis and global stability analysis of open flow configurations. For an extended review on modal analysis, the recent work of Taira et al.[139] illustrates different applications and scopes of this type of analysis.

In Section 2.2, we developed a general framework to compute the gradients (or sensitivities) of a selected global mode (both amplitude and frequency) obtained from the stability analysis of the linearized Navier-Stokes equations, under surface or parameter modifications. To do so, the mathematical approach necessary to perform gradient-based optimization loops oriented to flow control on hydrodynamic stability has been revisited to keep it under a limited and affordable computational cost. A discrete Lagrangian approach is introduced to calculate the sensitivity to baseflow modifications and to steady forcing, which is finally used to obtain the gradients related with a change in a set of flow parameters. The methodology is then extended to geometrical variations, implementing the surface mesh deformation as a control parameter on the Lagrangian formulation. By evaluating the residuals and the linearized Jacobian matrix of the flow with a modification in the surface mesh coordinates, we are able to obtain the gradients related with the surface mesh deformation. Similar work was done by Heuveline and Strauß[56], who implemented an SQP (Sequential Quadratic Programming) methodology for the optimization of the flow over a circular cylinder using finite differences in a continuous approach. However, due to the nature of their analysis, they required one evaluation per design variable (geometrical parameters in their case, each node in the surface mesh in our 
analysis). A major improvement of the present approach is that the methodology proposed here obtains, in only one evaluation, the information related to the sensitivity of every single mesh node in the surface. The gradients, containing independent information for each mesh node, are introduced into a steepest-descent algorithm[6] to control the behavior of the eigenvalue of interest. The steepest-descent algorithm aims to obtain the nearest local minimum of the desired functional, the amplification rate or frequency of the associated eigenvector. The methodology is checked on the well-known problem of the flow over a circular cylinder, where unsteady vortex shedding appears at Reynolds numbers (based on the cylinder diameter and the free stream velocity) beyond $R e=46$. Employing the proposed methodology, in a flow configuration of $R e=60$, the amplification rate of the most unstable eigenvalue is damped in a few iterations, stabilizing the wake with maximum local surface deformations of only $4 \%$ of the cylinder diameter. Larger deformations delay the onset of the instability in more than a $60 \%$ of the critical Reynolds number.

The rest of the Chapter is divided as follows. Section 6.2 reviews the mathematical modeling of the eigenvalue sensitivity to geometry changes, continuing in Section 6.3 with the implementation and validation of the algorithm with an analytic test case. Section 6.4 describes the case of study and contains the main contributions of this chapter, the sensitivity of the eigenvalue to geometrical changes. In Section 6.5 an optimization algorithm integrating the eigenvalue sensitivity is described. From Section 6.6 to 6.8 the results of the optimization are discussed, concluding on Section 6.10 with the main conclusions of this Chapter.

\subsection{Sensitivity of the eigenvalue to geometry changes}

As already remarked, stability analyses study the growth or decay of perturbations superimposed onto a usually steady solution of the Navier-Stokes equations. The analysis can identify which particular features are prone to evolve under slight modifications of the flow conditions, either by introducing a perturbation or caused by a modification of some physical or geometrical parameters. The growth of these features would give rise to a completely different flow configuration. The stability of the system can be analyzed solving the derived eigenvalue problem (Eq. 2.6), obtaining the relevant (physical) eigenmodes that would drive the system towards an unstable configuration.

Sensitivity analysis focuses on the response of the system to variations of certain parameters, either physical, geometrical or external perturbations, normally evaluated through the variation of an target functional. In our case, we are interested in the sensitivity of the eigenvalue associated to the particular eigenmode, normally unstable, and to assess how it will respond to the modification of chosen parameters or conditions of the flow configuration. The use of the foreseen sensitivities could be used on gradient-based optimization algorithms, but however the key-piece of the algorithm is to be described. Here we introduce the sensitivity of 
the eigenvalue with respect to changes in the surface geometry, which would allow to perform surface shape optimization based on the treatment of the global instability.

Our analysis is first focused on how the eigenvalues of the system, $\sigma \equiv \sigma(\overline{\mathbf{q}}(\mathbf{p}), \mathbf{p})$, will respond to variations of $\mathbf{p}$, the parameter (or parameters) of interest. We assume that our system of PDEs has a unique solution for each value of $\mathbf{p}$, and every variable is at least continuously differentiable.

The method of Lagrangian multipliers is used to calculate $\boldsymbol{\nabla}_{\mathbf{p}} \sigma$ for a range of parameters, considering as well the different physical constraints of the problem. In the most general framework, the Lagrangian functional is defined as:

$$
\mathcal{L}\left(\overline{\mathbf{q}}, \sigma, \hat{\mathbf{q}}, \mathbf{p}, \boldsymbol{\lambda}_{1}, \boldsymbol{\lambda}_{2}\right)=\sigma+<\boldsymbol{\lambda}_{1}, \mathbf{A}(\overline{\mathbf{q}}, \mathbf{p}) \hat{\mathbf{q}}-\sigma \hat{\mathbf{q}}>+<\boldsymbol{\lambda}_{2}, \mathbf{R}(\overline{\mathbf{q}}, \mathbf{p})>
$$

where, as it is shown in Section 2.2, $\boldsymbol{\lambda}_{1}$ and $\boldsymbol{\lambda}_{2}$ are the Lagrange multipliers that represents the adjoint eigenmode $\hat{\mathbf{q}}^{+}$and adjoint base flow $\overline{\mathbf{q}}^{+}$, respectively. Vector $\mathbf{p}$, of dimension $N_{p}$ contains those parameters of interest on the sensitivity analysis.

The gradient of the Lagrangian can be expressed as:

$$
\begin{aligned}
\nabla \mathcal{L}= & <\frac{\partial \mathcal{L}}{\partial \overline{\mathbf{q}}}, \delta \overline{\mathbf{q}}>+<\frac{\partial \mathcal{L}}{\partial \sigma}, \delta \sigma>+<\frac{\partial \mathcal{L}}{\partial \hat{\mathbf{q}}}, \delta \hat{\mathbf{q}}>+ \\
& <\frac{\partial \mathcal{L}}{\partial p}, \delta \mathbf{p}>+<\frac{\partial \mathcal{L}}{\partial \boldsymbol{\lambda}_{1}}, \delta \boldsymbol{\lambda}_{1}>+<\frac{\partial \mathcal{L}}{\partial \boldsymbol{\lambda}_{1}}, \delta \boldsymbol{\lambda}_{2}>
\end{aligned}
$$

The derivation with respect to the Lagrangian multipliers recovers the two constraints of the problem (base flow computation and eigenvalue problem), and the derivation with respect to the eigenmode leads to the adjoint eigenvalue problem, respectively. In addition, the derivation with respect to the eigenvalue leads to the dimensionalisation condition of the adjoint eigenmode $<\hat{\mathbf{q}}^{+}, \hat{\mathbf{q}}>=1$.

Cancelling the derivative with respect to the base flow, we recover the linear system

$$
\mathbf{A}(\overline{\mathbf{q}}, \mathbf{p})^{+} \overline{\mathbf{q}}^{+}=-\nabla_{\overline{\mathbf{q}}} \sigma
$$

with

$$
\begin{aligned}
\nabla_{\overline{\mathbf{q}}} \sigma & =\mathbf{B}^{+}(\overline{\mathbf{q}}, \hat{\mathbf{q}}, \mathbf{p}) \hat{\mathbf{q}}^{+} \\
\nabla_{\mathbf{q}_{f}} \sigma & =\overline{\mathbf{q}}^{+}
\end{aligned}
$$

as exposed in Section 2.2.2.

Finally, the derivative of the Lagrangian with respect to a change in the parameters is obtained via the expression:

$$
\begin{aligned}
<\frac{\partial \mathcal{L}}{\partial \mathbf{p}}, \delta \mathbf{p}> & =<\hat{\mathbf{q}}^{+}, \frac{\partial \mathbf{A}(\overline{\mathbf{q}}, \mathbf{p}) \hat{\mathbf{q}}}{\partial \mathbf{p}} \delta \mathbf{p}>+<\overline{\mathbf{q}}^{+}, \frac{\partial \mathbf{R}(\overline{\mathbf{q}}, \mathbf{p})}{\partial \mathbf{p}} \delta \mathbf{p}> \\
& =<\hat{\mathbf{q}}^{+}, \mathbf{B}_{\mathbf{p}}(\overline{\mathbf{q}}, \hat{\mathbf{q}}, \mathbf{p}) \delta \mathbf{p}>+<\overline{\mathbf{q}}^{+}, \mathbf{A}_{\mathbf{p}}(\overline{\mathbf{q}}, \mathbf{p}) \delta \mathbf{p}> \\
& =<\mathbf{B}_{\mathbf{p}}{ }^{+}(\overline{\mathbf{q}}, \hat{\mathbf{q}}, \mathbf{p}) \hat{\mathbf{q}}^{+}+\mathbf{A}_{\mathbf{p}}{ }^{+}(\overline{\mathbf{q}}, \mathbf{p}) \overline{\mathbf{q}}^{+}, \delta \mathbf{p}>
\end{aligned}
$$


As $\mathbf{p}$ is a vector of dimension $N_{p}, \mathbf{B}_{\mathbf{p}}(\overline{\mathbf{q}}, \hat{\mathbf{q}}, \mathbf{p})=\partial(\mathbf{A}(\overline{\mathbf{q}}, \mathbf{p}) \hat{\mathbf{q}}) / \partial \mathbf{p}$ and $\mathbf{A}_{\mathbf{p}}(\overline{\mathbf{q}}, \mathbf{p})=$ $\partial \mathbf{R}(\overline{\mathbf{q}}, \mathbf{p}) / \partial \mathbf{p}$ will be complex and real matrices, respectively, of dimension $\left(N_{v} \times\right.$ $N) \times N_{p}$, once the problem is discretised. With the term $\overline{\mathbf{q}}^{+}$present in the right hand side of (6.6), the calculation of the sensitivity to base flow modifications and forcing term are a necessary step to obtain the sensitivity to a change in the parameters.

Since the two last two constraints in the right hand side terms of Equation (6.1) are identically zero, the gradient of $\sigma$ with respect to variations of vector $\mathbf{p}$ can thus be obtained from:

$$
\Delta \sigma=<\nabla_{\mathbf{p}} \sigma, \delta \mathbf{p}>=<\frac{\partial \mathcal{L}}{\partial \mathbf{p}}, \delta \mathbf{p}>
$$

Expanding the inner products of Equations (6.6) and (6.7), and applying the adjoint operator definition, we finally arrive at:

$$
\boldsymbol{\nabla}_{\mathbf{p}} \sigma=\mathbf{M}^{-1} \mathbf{B}_{\mathbf{p}}{ }^{H}(\overline{\mathbf{q}}, \hat{\mathbf{q}}, \mathbf{p}) \mathbf{M} \hat{\mathbf{q}}^{+}+\mathbf{M}^{-1} \mathbf{A}_{\mathbf{p}}{ }^{H}(\overline{\mathbf{q}}, \mathbf{p}) \mathbf{M} \overline{\mathbf{q}}^{+} .
$$

To offer a comparison, the response of the eigenvalue respect to changes of the parameters of interest could be easily defined using the chain rule as:

$$
\frac{\mathrm{d} \sigma}{\mathrm{d} \mathbf{p}}=\frac{\partial \sigma}{\partial \overline{\mathbf{q}}} \frac{\partial \overline{\mathbf{q}}}{\partial \mathbf{p}}+\frac{\partial \sigma}{\partial \mathbf{p}}
$$

This equation is equivalent to (6.8), where the first term represents the variation induced by $\mathbf{p}$ on the base flow, and the second one the explicit dependence of the eigenvalue $\sigma$ with $\mathbf{p}$. Obviously, for a small number of parameters, it is possible and cheaper to apply the chain rule expression, Equation (6.9), to compute the sensitivity. However, for a large number of parameters (e.g. mesh nodes on a surface geometry), it can be easily checked that Equation (6.8) becomes more efficient as the number of parameters increases.

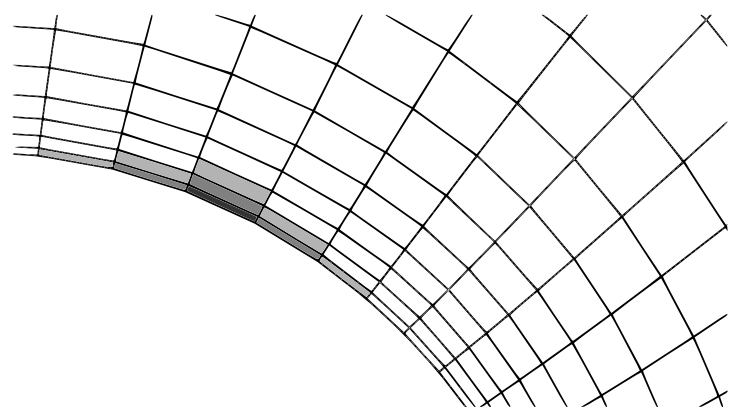

Figure 6.1: Compact second-order stencil used on the calculation of the Jacobian and the residuals. The dark grey cell indicates the wall cell that is deformed, grey cells correspond to the immediate neighbours, and light grey cells are the immediate next-neighbours.

In particular, we are interested in the effect that a displacement of a set of surface mesh nodes would have on a particular eigenvalue. To do that, we propose 
to substitute $\delta \mathbf{p}$ by the vector $\delta \mathbf{X}$, which contains the perturbation produced by the infinitesimal displacement of a set of surface mesh nodes. Vector $\delta \mathbf{X}$ has a leading dimension $N_{X}=N_{v} \times N$, but its only non-zero elements are those related with the displaced mesh nodes and those affected by the second order stencil of the numerical method (Fig. 6.1). $\boldsymbol{\nabla}_{\mathbf{p}} \sigma$, now renamed as $\boldsymbol{\nabla}_{\mathbf{X}} \sigma$, will then be a vector with the same dimension, $N_{X}$, containing information relative to the sensitivity of the eigenvalue to the displacement of a set of mesh nodes. Equation (6.8) is finally reformulated for simplicity as:

$$
\nabla_{\mathbf{X}} \sigma=\mathbf{M}^{-1} \mathbf{B}_{\mathbf{p}}(\overline{\mathbf{q}}, \hat{\mathbf{q}}, \mathbf{X})^{H} \mathbf{M} \hat{\mathbf{q}}^{+}+\mathbf{M}^{-1} \mathbf{A}_{\mathbf{p}}(\overline{\mathbf{q}}, \mathbf{X})^{H} \mathbf{M} \overline{\mathbf{q}}^{+}
$$

\subsection{Implementation and validation}

For the calculation of $\mathbf{A}_{\mathbf{p}}(\overline{\mathbf{q}}, \mathbf{X})$ and $\mathbf{B}_{\mathbf{p}}(\overline{\mathbf{q}}, \hat{\mathbf{q}}, \mathbf{X})$, relative to the perturbations due to geometrical changes, $\delta \mathbf{X}$, a mesh deformation of value $\epsilon_{2}$ is applied to the surface nodes of interest in their corresponding surface normal direction. The new residual vector and Jacobian matrix can be calculated using the new mesh (but keeping the old flow solution, $\overline{\mathbf{q}})$, such as $\mathbf{R}\left(\overline{\mathbf{q}}, \mathbf{X}+\epsilon_{2} \mathbf{d}\right)$ and $\mathbf{A}\left(\overline{\mathbf{q}}, \mathbf{X}+\epsilon_{2} \mathbf{d}\right)$, being $\mathbf{d}$ a vector of dimension $N_{v} \times N$ with the rows corresponding to the surface nodes set to 1 , and 0 in the other entries. The extension of this modification will only generate an effect on neighbor nodes affected by the second-order stencil of the code (Fig. 6.1). The value of $\epsilon_{2}$ should be small enough to not provoke a collapse of the first layer of the volume mesh, whose cells are characterized by having high aspect ratio. A detailed sensitivity study to the value of $\epsilon_{2}$ is presented on Section 6.3.1.

The calculation of the matrix $\mathbf{A}_{\mathbf{p}}(\overline{\mathbf{q}}, \mathbf{X})$ is then performed as:

$$
\mathbf{A}_{\mathbf{p}}(\overline{\mathbf{q}}, \mathbf{X})=\frac{\mathbf{R}\left(\overline{\mathbf{q}}, \mathbf{X}+\epsilon_{2} \mathbf{d}\right)-\mathbf{R}(\overline{\mathbf{q}}, \mathbf{X})}{\epsilon_{2}}
$$

while for the matrix $\mathbf{B}_{\mathbf{p}}(\overline{\mathbf{q}}, \hat{\mathbf{q}}, \mathbf{X})$ is taken:

$$
\mathbf{B}_{\mathbf{p}}(\overline{\mathbf{q}}, \hat{\mathbf{q}}, \mathbf{X})=\frac{\left(\mathbf{A}\left(\overline{\mathbf{q}}, \mathbf{X}+\epsilon_{2} \mathbf{d}\right)-\mathbf{A}(\overline{\mathbf{q}}, \mathbf{X})\right) \hat{\mathbf{q}}}{\epsilon_{2}}
$$

The resulting vectors, of Equations (6.11) and (6.12), only comprise non-zero elements in those positions related with the surface mesh nodes of interest and their stencil. In the cases where only one mesh node is considered for the sensitivity analysis, Equation (6.10) will give the sensitivity of the examined eigenvalue to normal displacement of this particular mesh node. Repeating this process for every single mesh node would give singular information for each surface point, but this process would be extremely expensive in computational terms. The ideal case is to obtain all that information in one single evaluation. To this aim, we propose the following. If more than one mesh node is displaced at a time, and $\mathbf{A}_{\mathbf{p}}(\overline{\mathbf{q}}, \mathbf{X})$ and $\mathbf{B}_{\mathbf{p}}(\overline{\mathbf{q}}, \hat{\mathbf{q}}, \mathbf{X})$ are input vectors in Equation (6.10), the final result will be a 


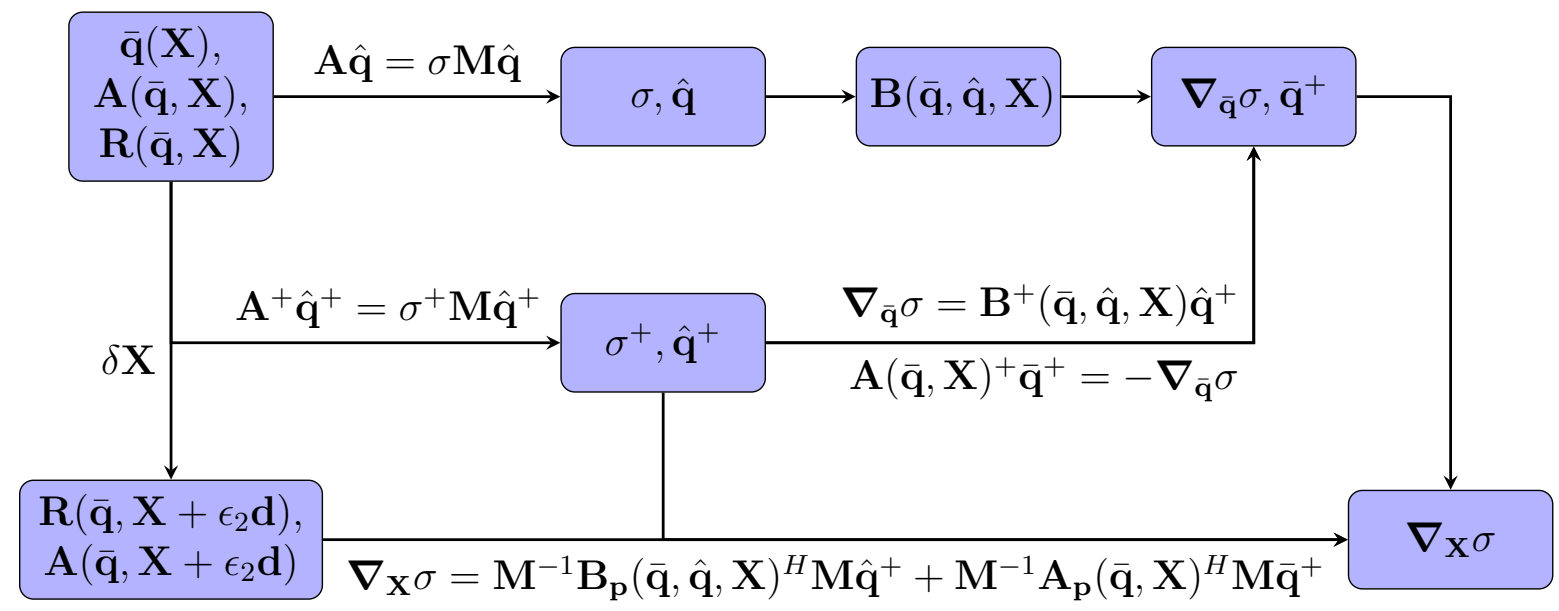

Figure 6.2: Mathematical procedure for the calculation of the sensitivity to mesh nodes displacements.

scalar, obtaining only a "general" sensitivity of the eigenvalue to the displacement of that set of nodes. In order to preserve the information relative to every single mesh node, each vector is stored in the diagonal of a square matrix with leading dimension $N_{v} \times N$. This mathematical strategy allows to recover a matrix structure for $\mathbf{A}_{\mathbf{p}}(\overline{\mathbf{q}}, \mathbf{X})$ and $\mathbf{B}_{\mathbf{p}}(\overline{\mathbf{q}}, \hat{\mathbf{q}}, \mathbf{X})$, and eventually obtain a sensitivity vector $\boldsymbol{\nabla}_{\mathbf{X}} \sigma$ with information relative to all the displaced mesh nodes, in just one evaluation.

To sum up, the procedure to obtain the sensitivity gradients to geometrical changes is depicted in Figure 6.2, which can be itemized in the following steps:

1. Calculate the base flow, $\overline{\mathbf{q}}$, using the Navier-Stokes solver;

2. Compute the direct global modes, $\hat{\mathbf{q}}$, based on the discrete linearized Jacobian, $\mathbf{A}(\overline{\mathbf{q}}, \mathbf{X})$;

3. Compute the adjoint global modes, $\hat{\mathbf{q}}^{+}$, based on the discrete adjoint operator of the Jacobian, $\mathbf{A}^{+}(\overline{\mathbf{q}}, \mathbf{X})=\mathbf{M}^{-1} \mathbf{A}(\overline{\mathbf{q}}, \mathbf{X}) \mathbf{M}$

4. Calculate the sensitivity to base flow modifications, $\nabla_{\overline{\mathbf{q}}} \sigma=\mathbf{B}^{+}(\overline{\mathbf{q}}, \hat{\mathbf{q}}, \mathbf{X}) \hat{\mathbf{q}}^{+}$;

5. Solve the linear system $\mathbf{A}(\overline{\mathbf{q}}, \mathbf{X})^{+} \overline{\mathbf{q}}^{+}=-\nabla_{\overline{\mathbf{q}}} \sigma$ to obtain the sensitivity to steady forcing, $\overline{\mathbf{q}}^{+}$

6. Extract the residuals vector of the flow solution, $\mathbf{R}(\overline{\mathbf{q}}, \mathbf{X})$;

7. Displace the surface mesh nodes in normal direction by a small distance $\epsilon_{2} \ll 1$;

8. Compute the new residuals vector and Jacobian matrix, $\mathbf{R}\left(\overline{\mathbf{q}}, \mathbf{X}+\epsilon_{2} \mathbf{d}\right)$ and $\mathbf{A}\left(\overline{\mathbf{q}}, \mathbf{X}+\epsilon_{2} \mathbf{d}\right)$, followed by the matrix $\mathbf{B}_{\mathbf{p}}(\overline{\mathbf{q}}, \hat{\mathbf{q}}, \mathbf{X})$ and the vector $\mathbf{A}_{\mathbf{p}}(\overline{\mathbf{q}}, \mathbf{X})$, using Equations (6.11) and (6.12);

9. Calculate the sensitivity to geometrical changes, $\boldsymbol{\nabla}_{\mathbf{X}} \sigma$, using Equation 6.10. 


\subsubsection{Analytic test case}

To check the validity of our expressions, we consider here a simplified analytic test case with two flow variables, $u$ and $v$, and one parameter $p$. Therefore, $\mathbf{q}=(u, v, p)$ is the state vector, and the corresponding flux vector defined by the non-linear system:

$$
\mathbf{R}(\mathbf{q}, p)=\left[\begin{array}{c}
e^{p u v}-1 \\
u p^{2}+v+1
\end{array}\right]
$$

and the system mass matrix arbitrarily defined as:

$$
\mathbf{M}=\left[\begin{array}{cc}
0.25 & 0.0 \\
0.0 & 0.5
\end{array}\right]
$$

System (6.13) has a point of equilibrium (base flow) $\overline{\mathbf{q}}=(\bar{u}, \bar{v}, p)=\left(-1 / p^{2}, 0, p\right)$, for which $\mathbf{R}(\overline{\mathbf{q}}, p)=\mathbf{0}$, upon which the stability analysis is computed. The analytic Jacobian matrix with respect to that base flow $(\bar{u}, \bar{v})$ can be easily calculated:

$$
\mathbf{A}(\overline{\mathbf{q}}, p)=\left[\frac{\mathrm{d} \mathbf{R}(\overline{\mathbf{q}}, p)}{\mathrm{d} \mathbf{q}}\right]_{\mathbf{q}=\overline{\mathbf{q}}}=\left[\begin{array}{cc}
p v e^{p u v} & p u e^{p u v} \\
p^{2} & 1
\end{array}\right]_{\mathbf{q}=\overline{\mathbf{q}}}=\left[\begin{array}{cc}
0 & -1 / p \\
p^{2} & 1
\end{array}\right] .
$$

When we consider the generalized eigenvalue problem $\mathbf{A}(\overline{\mathbf{q}}, p) \hat{\mathbf{q}}=\sigma \mathbf{M} \hat{\mathbf{q}}$, the eigenvalues are $\sigma_{1,2}=(1 \pm \sqrt{1-8 p})$. After some calculations, the direct and adjoint global modes, $\hat{\mathbf{q}}^{+}, \overline{\mathbf{q}}^{+}$and matrices $\mathbf{B}_{\mathbf{p}}(\mathbf{q}, \hat{\mathbf{q}}, p), \mathbf{A}_{\mathbf{p}}(\mathbf{q}, p)$ are obtained:

$$
\begin{aligned}
\mathbf{B}(\mathbf{q}, \hat{\mathbf{q}}, p) & =\left[\begin{array}{cc}
e^{p u v} p^{2}\left[\hat{\mathbf{q}}_{1} v^{2}+\hat{\mathbf{q}}_{2}(u v+1 / p)\right] & e^{p u v} p^{2}\left[\hat{\mathbf{q}}_{2} u^{2}+\hat{\mathbf{q}}_{1}(u v+1 / p)\right] \\
0
\end{array}\right]_{\mathbf{q}=\overline{\mathbf{q}}} \\
& =\left[\begin{array}{cc}
p & {[2+p(1+\sqrt{1-8 p})] / 2 p} \\
0 & 0
\end{array}\right], \\
\mathbf{B}_{\mathbf{p}}(\mathbf{q}, \hat{\mathbf{q}}, p) & =\left[\begin{array}{c}
e^{p u v}\left(v \hat{\mathbf{q}}_{1}+u \hat{\mathbf{q}}_{2}\right)(p u v+1) \\
2 p \hat{\mathbf{q}}_{1}
\end{array}\right]_{\mathbf{q}=\overline{\mathbf{q}}}=\left[\begin{array}{c}
-1 / p^{2} \\
-8 /(1-\sqrt{1-8 p})
\end{array}\right], \\
\mathbf{A}_{\mathbf{p}}(\mathbf{q}, p) & =\left[\begin{array}{c}
u v e^{p u v} \\
2 p u
\end{array}\right]_{\mathbf{q}=\overline{\mathbf{q}}}=\left[\begin{array}{c}
0 \\
-2 / p
\end{array}\right],
\end{aligned}
$$

where $\hat{\mathbf{q}}=\left(\hat{\mathbf{q}}_{1}, \hat{\mathbf{q}}_{2}\right)$ is the associated eigenvector. Finally, to compute the sensitivity of the eigenvalue $\sigma_{1}$ to changes in $p$ (in this case, it can either be computed analytically using the chain rule, or the Lagrangian approach), we use:

$$
\boldsymbol{\nabla}_{p} \sigma_{\text {Analytic }}=\frac{\mathrm{d} \sigma_{1}}{\mathrm{~d} p}=\frac{\partial \sigma_{1}}{\partial \overline{\mathbf{q}}} \frac{\partial \overline{\mathbf{q}}}{\partial p}+\frac{\partial \sigma_{1}}{\partial p}=-\frac{4}{\sqrt{1-8 p}} .
$$

At this point, we select a range for $p>1 / 8$ such that the resulting eigenvalues are always complex. We evaluate the sensitivity of the numerical methodology 


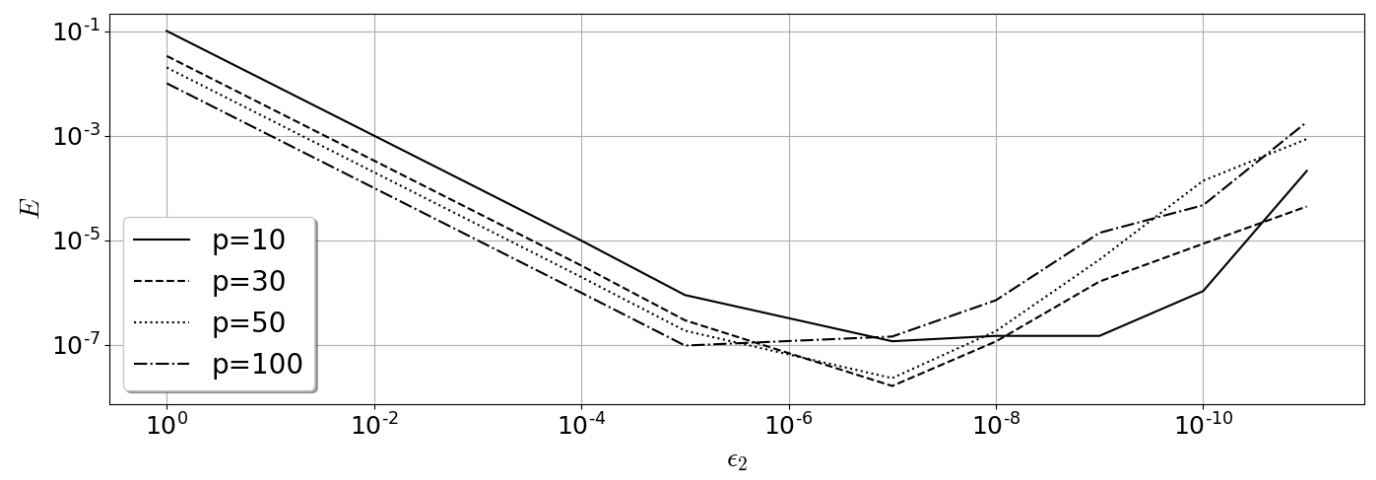

Figure 6.3: Comparison between the analytic and numerical sensitivity of the eigenvalue to changes in $\epsilon_{2}$ and the parameter $p$.

described on this Section to the values chosen for the $\epsilon_{2}$ parameter. To do so, the relative error is calculated as

$$
E=\left|\frac{\nabla_{p} \sigma_{\text {Analytic }}-\nabla_{p} \sigma_{\text {Numerical }}}{\nabla_{p} \sigma_{\text {Analytic }}}\right|
$$

where $\nabla_{p} \sigma_{\text {Numerical }}$ has been calculated with the numerical version of matrices $\mathbf{B}_{\mathbf{p}}(\mathbf{q}, \hat{\mathbf{q}}, p), \mathbf{A}_{\mathbf{p}}(\mathbf{q}, p)$ using Equations (6.11) and (6.12). Results are shown in Figure 6.3 , where it can be observed that optimal accuracy is reached for values of $\epsilon_{2}$ of order $\mathcal{O}\left(10^{-7}\right)$, and that slight variations of $\epsilon_{2}$ will still keep the error low.

\subsection{Numerical experiment. The cylinder wake in- stability}

The first instability of the cylinder wake has been widely investigated using global stability analysis[12, 21, 46, 77, 97]. For a critical Reynolds number (Re $\approx 47$ ), an anti-symmetrical global mode becomes unstable, increasing the intensity of the perturbations inside the recirculation region of the wake until its symmetry breakes, developing the wide-known 'Von-Kármán' vortex street. We revisit this case as a two-dimensional laminar flow numerical experiment, aiming to evaluate the behavior of the cylinder wake global mode, when the body surface is modified, using the global mode sensitivity gradients approach proposed.

A rectangular domain was considered for the generation of an $\mathrm{H}$-grid (Fig. 6.4-(a)). A mesh convergence analysis for the baseflow calculation was performed, showing a mesh independent drag coefficient (up to four digits) when used the retained mesh, but it is not included here by conciseness. A reference Reynolds number equal to 60 , with a developed unsteady vortex shedding, was considered for the test. To obtain a symmetrical steady base flow, only the upper half of the 


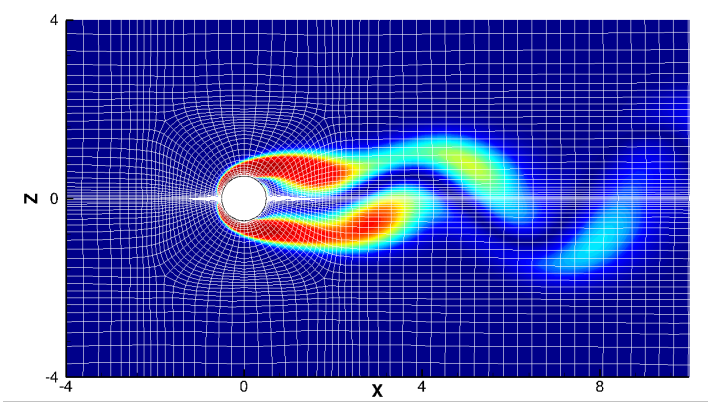

(a)

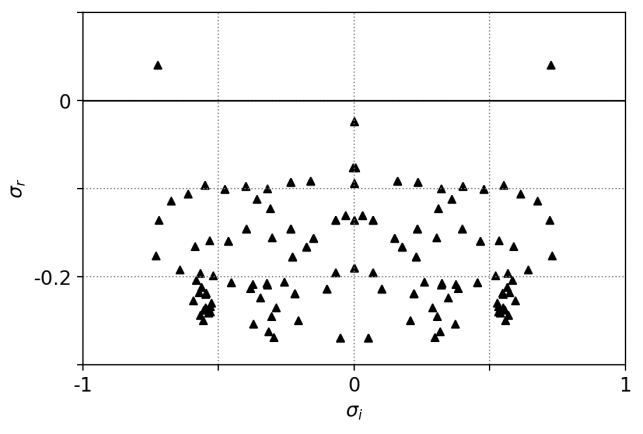

(b)

Figure 6.4: (a): Unstructured H-Grid used for the simulations, overlapped with vorticity magnitude contours $(R e=60)$. Only half of the mesh nodes are shown. (b): Eigenvalue distribution for the laminar flow over a cylinder, $R e=60$.

$\mathrm{XZ}$ plane is considered on the numerical simulation to ensure a symmetrical flow condition.

The linearized Jacobian matrix was extracted from the solution using a discrete approach and fed into the eigenvalue solver. The spectra of this matrix (Fig. 6.4(b)) shows a pair of complex eigenvalues with positive real part. This unstable pair of eigenvalues is associated to a Hopf bifurcation, characteristic of the Von-Karman street that can be observed if the flow solution is integrated over time using a transient solver (Fig. 6.10-(a)).

The sensitivity of the unstable eigenvalue to base flow modifications and steady forcing $\left(\overline{\mathbf{q}}^{+}\right)$are calculated for a Reynolds number of $R e=60$ using the derived expressions[77]:

$$
\begin{aligned}
\nabla_{\overline{\mathbf{q}}} \sigma & =\mathbf{B}^{+}(\overline{\mathbf{q}}, \hat{\mathbf{q}}, \mathbf{X}) \hat{\mathbf{q}}^{+}, \\
\nabla_{\mathbf{q}_{f}} \sigma & =\overline{\mathbf{q}}^{+} .
\end{aligned}
$$

Finally, as the main contribution of this chapter, the sensitivity to normal displacement of the surface nodes can be calculated, as stated in Equation (6.10). We plot the values of the sensitivity against the azimuth coordinate on Figure 6.5, together with a geometrical representation of the sensitivity gradients over the cylinder circular shape. The real part of the gradient is directly related with the real part of the eigenvalue, meaning that normal displacements of the nodes following the positive direction of the sensitivity have a destabilizing impact on the eigenvalue. In addition, the information related to the imaginary part of the gradient is linked to the associated frequency of the eigenvalue (its pulsation, $\sigma_{i}=2 \pi S t$ ), where normal displacements will have an increment or decrement effect on the pulsation, depending on the sign of the gradient. As can be observed in the figure, the regions located close to $90^{\circ}$ (considering $0^{\circ}$ as the stagnation point) are the most sensitive in the amplification rate to surface deformations, where a deformation in the outward normal direction implies a further destabilization of the global mode, together 
with a decrement on the associated frequency. On the contrary, modifications at the frontal and rear stagnation areas $\left(0^{\circ}\right.$ and $180^{\circ}$, respectively) have minor effect on the eigenvalue.

The value of the gradient at each surface node shows the contribution to the real/imaginary part of the eigenvalue if that node has a displacement in the normal direction. The real part of the sensitivity gradients, Figure 6.5-(b), shows mainly positive values so, from the integration of the area behind the curve of Figure 6.5(a), it can be inferred that an increment in the radius of the cylinder will have a destabilizing effect, as it was expected, since this can be associated to an increment of the Reynolds number. The imaginary part of the sensitivity, Figure 6.5-(c), shows however positive and negative values. Modifications following this gradient would led to a more slender body, either in the direction of the flow, or perpendicular to it, and with higher or lower associated frequencies, respectively.

In the following sections, we propose an algorithm in which we use these sensitivities to drive a shape optimization process to control the cylinder wake first instability. Following this procedure, deformations of the surface of the body following the normal direction of the surface nodes are applied at each optimization step according to the normalized sensitivity gradients, with the maximum deformation controlled by the $\alpha$ parameter, Equation (6.23), proportional to a percentage of the cylinder original diameter. At each iteration, the computational mesh is deformed and a new base flow, stability analysis and sensitivity gradients are computed. Due to the nature of this study, the only geometrical restriction imposed to the mesh deformation is to keep the symmetry of the body with respect to the XY plane. The process is limited to a maximum number of 25 iterations, to avoid larger mesh deformations that would result in the divergence of the flow solver due to the decay on the mesh quality. The purpose of the following sections is therefore not an optimal optimization of the cylinder shape, but a validation of the eigenvalue sensitivity gradients, showing its potential to control the leading instability by surface deformations.

\subsection{Optimization process}

The calculated surface gradients allow to perform a gradient-based optimization, using the least stable eigenvalue as the cost functional. For the sake of simplicity, a steepest-descent algorithm[6] is used to guide the process, where each new configuration is defined as:

$$
\mathbf{X}^{n+1}=\mathbf{X}^{n}+\alpha \cdot \operatorname{Re}\left(\boldsymbol{\nabla}_{\mathbf{X}} \sigma\right) \text { or } \mathbf{X}^{n+1}=\mathbf{X}^{n}+\alpha \cdot \operatorname{Im}\left(\boldsymbol{\nabla}_{\mathbf{X}} \sigma\right)
$$

with the use of the real or imaginary part of the gradient, depending on which part of the complex eigenvalue is considered as the cost function, and where $\alpha$ is a userdefined parameter, that allows to damp the gradients and control the optimization process. To have a better control of the damping parameter $\alpha$, the gradients were 


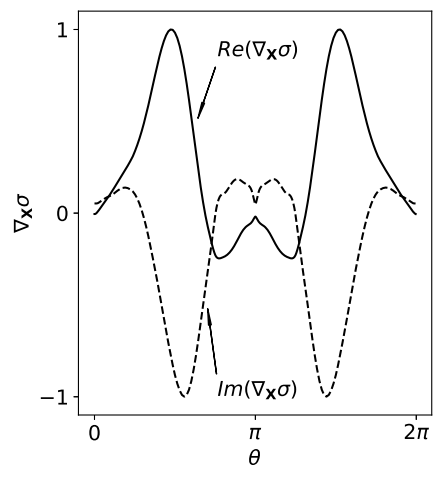

(a)

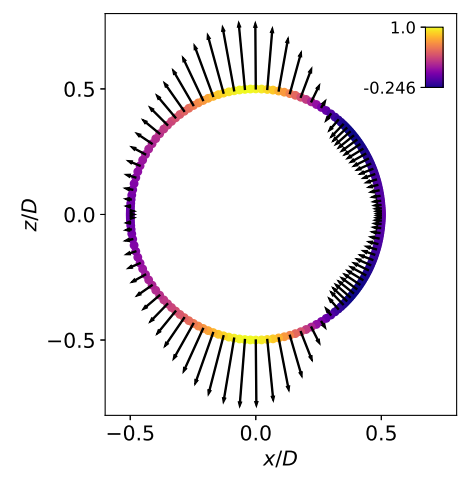

(b)

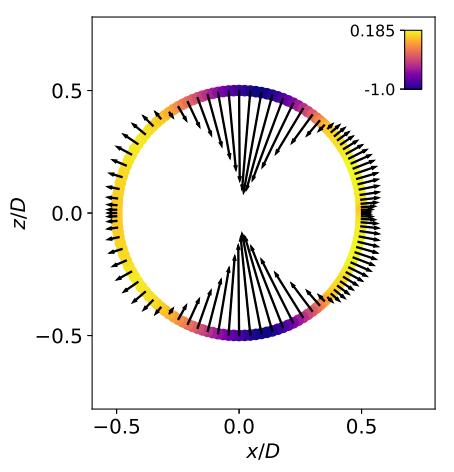

(c)

Figure 6.5: Sensitivity of the eigenvalue to outward normal displacements of the surface nodes, as a function of the circular cylinder azimuth (left). Node distribution of the sensitivity of the amplification rate (center) and the sensitivity of the pulsation (right) of the eigenvalue.

normalized with their maximum absolute value. The deformation of the mesh was performed through the deformation module present in the DLR TAU-code, which uses Radial Basis Function techniques to preserve the integrity of the mesh while accomplishing the requested surface mesh node displacements.

At each stage of the optimization, the base flow and its corresponding eigenvalues and eigenvectors are recalculated, obtaining the new spectra and to select the global mode of interest. As a relatively large number of eigenvalues is calculated during each iteration, it is necessary to "track" the least stable eigenvalue that is defined as the cost functional for the optimization process. This is done using a Modal Assurance Criterion (MAC)[102], in which a "seed" is defined at the beginning of the process, and used to evaluate the orthogonality of the newly calculated eigenmode, $\hat{\mathrm{q}}_{\mathrm{i}}$ with the reference one, $\hat{\mathrm{q}}_{\mathrm{j}}$ :

$$
\operatorname{MAC}\left(\hat{\mathbf{q}}_{\mathbf{i}}, \hat{\mathbf{q}}_{\mathbf{j}}\right)=\left(\frac{\left|\hat{\mathbf{q}}_{\mathbf{i}} \cdot \hat{\mathbf{q}}_{\mathbf{j}}\right|}{\left\|\hat{\mathbf{q}}_{\mathbf{i}}\right\|\left\|\hat{\mathbf{q}}_{\mathbf{j}}\right\|}\right)^{2}
$$

In the MAC indicator index approach, a value of 1 is found when the pair of eigenmodes are linearly dependent (or identical), and is zero for orthogonal eigenmodes (not similar shapes).

To sum up, starting from a converged base flow and a selected eigenvalue (the least stable or more stable) as the cost function, the optimization process (depicted in Figure 6.6) can be summarized as:

1. Calculate the sensitivity to geometrical changes;

2. Deform the surface mesh according to the gradients, as $\mathbf{X}^{n+1}=\mathbf{X}^{n}+\alpha$. $\operatorname{Re}\left(\boldsymbol{\nabla}_{\mathbf{X}} \sigma\right)$ or $\mathbf{X}^{n+1}=\mathbf{X}^{n}+\alpha \cdot \operatorname{Im}\left(\boldsymbol{\nabla}_{\mathbf{X}} \sigma\right) ;$ 
3. Calculate the new base flow and its corresponding eigenvalues and eigenmodes from the linearized Navier-Stokes solution;

4. Identify and evaluate the new eigenvalue. If the maximum number of iterations has been reached, stop the process. If not, loop again.

\subsection{Control of the growth rate of the instability}

The control of the vortex shedding global instability is typically of major interest. To probe the capability of the sensitivity gradients developed in this work, we aim at modifying the growth rate of the instability associated eigenvalue and with it the onset of the cylinder wake breakdown, by means of local modifications of the circular surface.

To stabilize the eigenvalue (making its real part negative), the deformations at each iteration of the optimization process are guided in the reverse direction of the real part of the sensitivity gradients. For the particular case of taking deformations of $1 \%$ of the cylinder diameter for each iteration, the pair of complex eigenvalues become stable after only 5 deformation steps (Fig. 6.7-(a)). The amplification rate is damped from an initial value of 0.0412 to -0.0182. A consequence of the deformation is also an increment on the pulsation, as suggested by Figure 6.5-(c). Its final value is 0.933, compared to an initial pulsation of 0.725 . The final shape, is tested with non-steady simula-

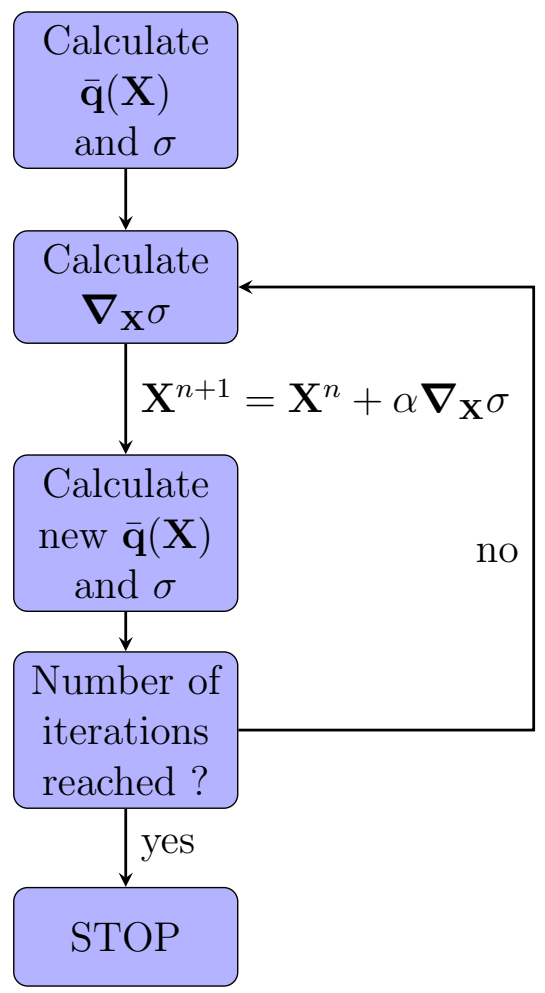

Figure 6.6: Optimization loop. tions, showing a non-oscillating wake behavior for a Reynolds number value of 60 (Fig. 6.10-(b)). Indeed, the appearance of the instability has been delayed up to a Reynolds number of $R e=65$ (Fig. 6.7-(a)).

However, if the optimization process is guided towards a more unstable eigenvalue (larger positive real part), deformations in the direction of the gradients should be included. The optimization process can only complete 11 iterations before the solver is not able to converge to a steady solution (essential requirement for the stability analysis approach followed here). For this final configuration, the onset of the instability is pushed down to an early Reynolds number of 36, with an amplification rate of 0.0685 and an associated pulsation of 0.590 at $\mathrm{Re}=60$.

The final shapes obtained after the optimization are shown in Figure 6.7-(b). It can be seen that when pursuing a more stable wake, the body is transformed into 


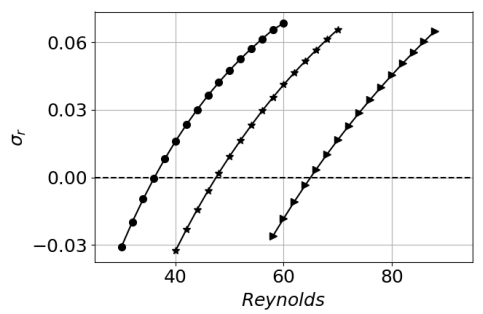

(a)

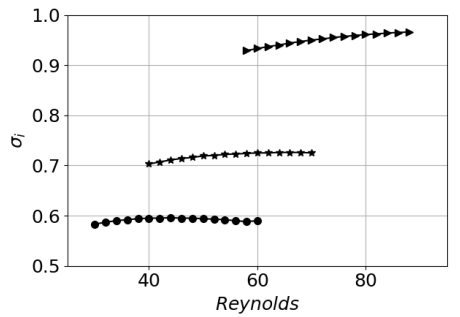

(b)

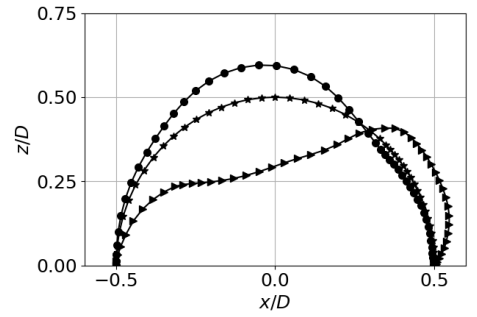

(c)

Figure 6.7: Eigenvalue real and imaginary parts for the reference and optimized geometries (left and center images), and final shapes of the body after the optimization (right). $-*$ : reference; $-\bullet$ : minimum amplification rate; $-\bullet$ : maximum amplification rate.

a more slender body, whereas the destabilization of the wake leads to an increment on the frontal area of the body.

\subsection{Control of the vortex shedding associated fre- quency}

In this section we apply the methodology to control the oscillation frequency. This implies the control the imaginary part of the eigenvalue, and therefore the associated frequency of the physical phenomenon, using the imaginary part of the sensitivity gradients.

After 25 iterations, the imaginary part of the eigenvalue is increased to a value of 0.946 (Fig. 6.8-(b)). The final shape achieved is similar to a reversed water droplet, with a smaller frontal area. The rear part of the cylinder is only slightly modified, but the node-by-node surface deformation followed on this approach led to a visible decrement on the quality on the mesh in this area.

Interestingly, the algorithm used for this gradient-based optimization did not work well when seeking a minimum associated frequency. The pulsation stacked in a local minimum of the design space, and the mesh deformations quickly led to a divergence of the solver without a further improvement on the increment of the eigenvalue pulsation. To reduce this interference, we constrained the deformations of the rear part of the body, keeping some surface nodes without displacement. With this constraint the optimization could be completed. A local minimum was reached after 12 iterations, with a final pulsation of 0.682 . The related frequency evolution with the Reynolds number for the optimized configurations, and the final body shapes, are plotted in Figure 6.8. 


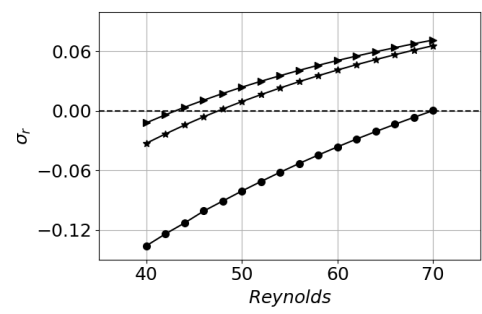

(a)

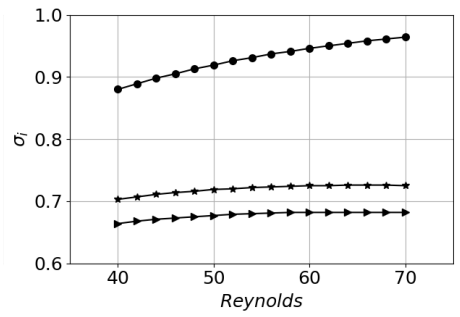

(b)

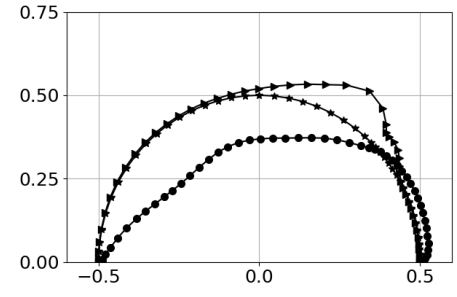

(c)

Figure 6.8: Eigenvalue real and imaginary parts for the reference and optimized geometries (left and center images), and final shapes of the body after the optimization (right). $-*$ : reference; $\rightarrow$ : minimum pulsation; $-\bullet$ : maximum pulsation.

\subsection{Discussion on the influence of the deforma- tion control parameter, $\varepsilon$}

The consistency of the methodology was tested using different deformation parameters to ensure a convergence of the optimization method. Deformations in each node of $0.25,0.5,1.0,1.5$ and $2.0 \%$ of the cylinder diameter are tested when seeking a more stable eigenvalue. As in Section 6.6, the real part of the geometrical sensitivity gradient is used to guide the local deformations.

The stopping criterion is based on the rate of change of the real part of the eigenvalue during the process:

$$
\left|\sigma_{r}^{(n)}-\sigma_{r}^{(n-1)}\right| \leq \frac{\left|\sigma_{r}^{(n-1)}-\sigma_{r}^{(n-2)}\right|}{4},
$$

which allows to have a low rate of change for few iterations, detecting the apparition of a local minimum. If the rate of change of the eigenvalue changed sign (pushing the eigenvalue to a less stable value), the optimization process would also stop.

Results of the convergence study are shown in Figure 6.9, where it can be seen that the final amplification rate is similar for all the cases. In every case, the eigenvalue crosses the imaginary axis after a maximum deformation in any node of less than $5 \%$ of the original cylinder diameter. In the same figure, we also plot the final shape after the optimization process for each deformation $\alpha$. All the shapes fall within a confidence interval defined as three times the standard deviation of the results (3stdev) of the final shape.

\subsection{Analysis and discussion}

The final geometries reached during the optimization process are plotted on Figures 6.7-(c) and 6.8-(c). Besides, Table 6.1 shows the different values of the eigenvalue 


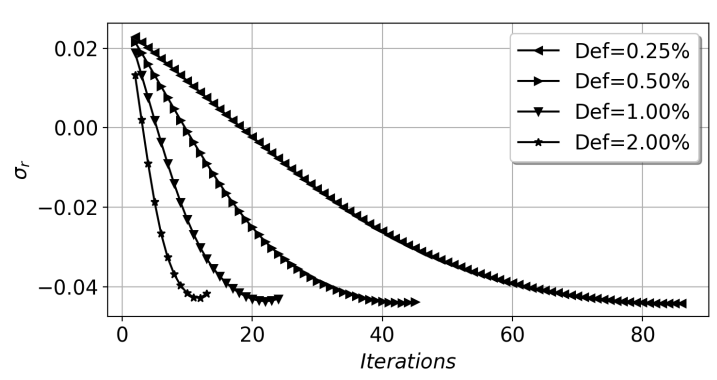

(a)

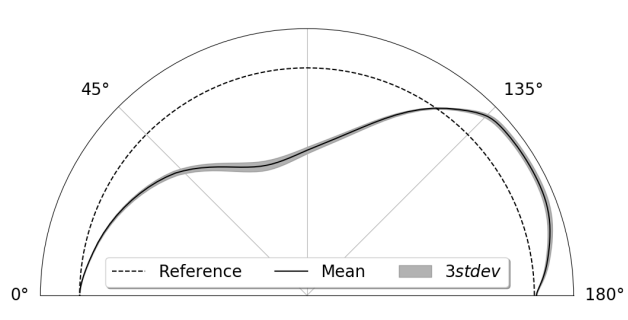

(b)

Figure 6.9: Convergence study for difference deformation ratios. (a) Behavior of the real part of the eigenvalue with respect to the number of iterations depending on the deformation ratio, and (b) final shape after the optimization process. 3stdev denotes three standard deviations.

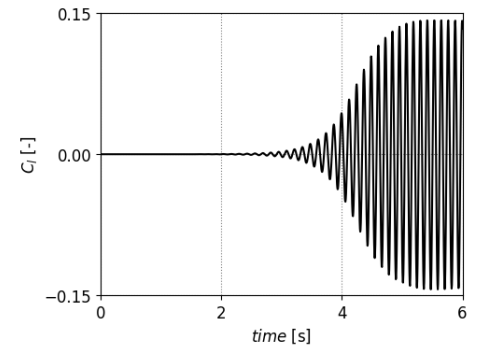

(a)

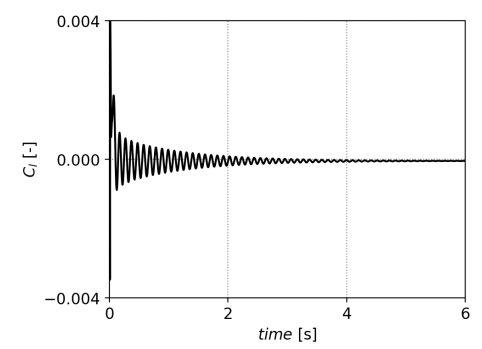

(b)

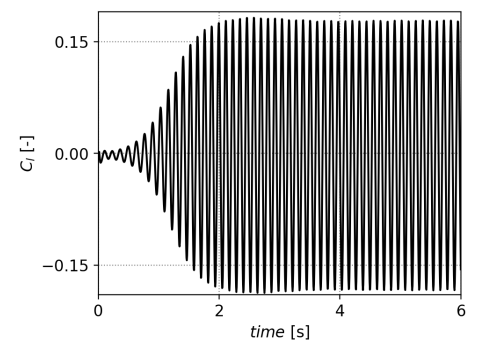

(c)

Figure 6.10: Non-steady solutions for a $R e=60$ laminar flow configuration. Lift coefficient monitors for (a) reference cylinder, (b) optimized shape for minimum amplification rate and (c) optimized shape for minimum frequency.

related with the cylinder wake global mode at $R e=60$ for the different configurations. This outlines the relation of the amplification rate and the pulsation with the geometrical shape for the wake instability. For illustration, a comparison of the flow solutions at $R e=60$ has also been included in Figures 6.11 and 6.12, where streamwise velocity contours of a symmetric flow solution are shown.

It was observed that the optimization process when seeking minimum amplification rate or maximum associated frequencies reduces the frontal area of the cylinder, decreasing the radius of curvature of the frontal part of the body and creating more slender shape. On the contrary, reduced frequencies and larger amplification rates are associated with large frontal areas.

In Figure 6.7-(a), the new critical Reynolds values for the two deformed configurations obtained in the search for a minimum/maximum amplification rate can be quantified and compared with the reference eigenvalue curve. The critical values for the modified geometries are $R e=65$ and $R e=36$, respectively. This is a delay/anticipation on the apparition of the vortex instability of approximately $41 \% / 22 \%$ on 
Table 6.1: Summary of amplification rate and pulsation values for $R e=60$.

\begin{tabular}{|l|c|c|c|c|}
\hline Methodology & Gradient used & $\sigma_{r}$ & $\sigma_{i}$ & $S t$ \\
\hline Reference & & 0.041 & 0.725 & 0.115 \\
\hline Min. Amp. rate & $-\operatorname{Re}\left(\boldsymbol{\nabla}_{\mathbf{X}} \sigma\right)$ & -0.018 & 0.933 & 0.148 \\
Max. Amp. rate & $\operatorname{Re}\left(\boldsymbol{\nabla}_{\mathbf{X}} \sigma\right)$ & 0.069 & 0.590 & 0.094 \\
Min. Frequency & $-\operatorname{Im}\left(\boldsymbol{\nabla}_{\mathbf{X}} \sigma\right)_{\text {modified }}$ & 0.051 & 0.682 & 0.109 \\
Max. Frequency & $\operatorname{Im}\left(\boldsymbol{\nabla}_{\mathbf{X}} \sigma\right)$ & -0.036 & 0.946 & 0.150 \\
\hline
\end{tabular}
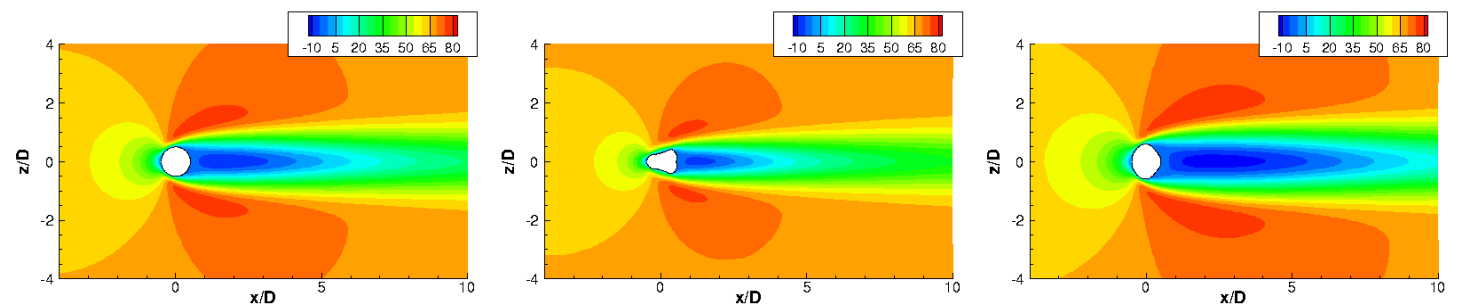

Figure 6.11: Streamwise velocity contours for a symmetrical steady flow. Final optimized surface shapes for a minimum(center)/maximum(right) eigenvalue amplification rate, at $R e=60$. Contours for the reference geometry are plotted on the left picture.
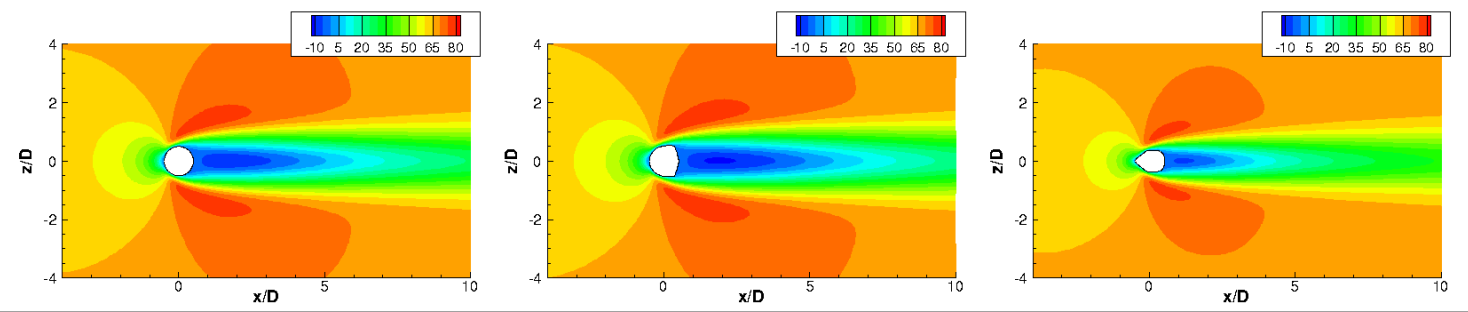

Figure 6.12: Streamwise velocity contours for a symmetrical steady flow. Final optimized surface shapes for a minimum(center)/maximum(right) eigenvalue pulsation, at $R e=60$. Contours for the reference geometry are plotted on the left picture.

the Reynolds number. From Figure 6.8-(a), similar data can be extracted for the associated Strouhal number of the instability when seeking minimum/maximum eigenvalue associated frequency, which has been calculated from the imaginary part of the eigenvalue $\left(S t=\sigma_{i} / 2 \pi\right)$. For a Reynolds number of 70 , unstable regime for the three cases, an decrement/increment of $6 \% / 33 \%$ was registered. This difference in frequency can be related to the constrained deformations.

\subsection{Conclusions}

Global stability analysis recovers the eigenvalues and eigenmodes that can drive the behavior of the hydrodynamic system. Using a Lagrangian methodology, and a dis- 
crete formulation approach, a sensitivity gradient to geometrical changes has been developed, and used to drive a shape optimization process. This eigenvalue-based gradient can be used with the goal of controlling global instabilities. This novel and simple approximation results in accurate gradients that predict the behavior of the eigenvalue related with a global eigenmode. Moreover, the proposed methodology allows to obtain the gradient of each surface mesh node in just one single computation step, providing valuable and singular information with minimal computational cost. At each surface point, the obtained gradients contain information about the growth rate and the frequency parameters of the global instability. The modification of the geometry according to these gradients enables the control of such instabilities, either promoting or damping them, or altering its related frequency. The discrete nature of the gradient, with information relative to each node, allows to perform local deformations on the surface mesh. The combination of local deformations with Radial Basis Functions mesh deformation techniques drives the aerodynamic optimization of a geometry with the eigenvalue of the dominant global mode (Hopf bifurcation) as the cost function.

The proposed methodology has been tested with the well-known global instability of the cylinder wake. Despite the simplicity of the optimizing algorithm used in this work, an excellent behavior on the suppression of the oscillating behavior of the wake has been shown, delaying the onset of the instability up to a $41 \%$ of the initial critical value for the Reynolds number for a fixed number of optimization steps. In a similar way, the cylinder shape has been altered to promote the apparition of vortex shedding, anticipating the onset by $22 \%$. In addition, frequency control for the instability was also tested, for situations where the flow oscillation does not need to be suppressed, but controlled. The methodology proposed here looks forward to establish the basis of an eigenvalue-based surface optimization at an affordable cost. Furthermore, the use of a compressible code for the analysis shows the potential of the algorithm to be used in more complex and realistic scenarios. 



\section{Part V}

\section{Conclusions and Future Directions}





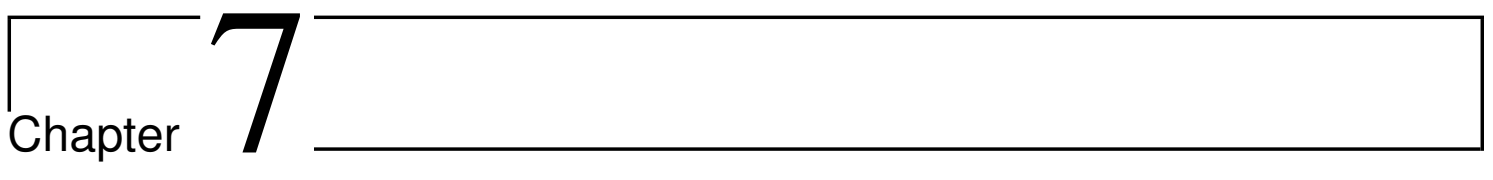

\section{Final Discussion and Work Perspectives}

\section{Discussion}

The objective of this thesis was the application of linear stability analysis by means of direct and adjoint methods to turbulent complex scenarios with highly detached flows, improving and extending the capabilities of an associated sensitivity analysis.

Flow solutions were obtained using a Finite Volume approximation through an industrial CFD solver, the DLR-TAU Code. Only minor modifications had to be done to the flow solver, mainly related with input-output formats. On the current trend of using High-Order methodologies (based on Discontinuous-Galerkin approaches, for example), using a Finite Volume solver may seem behind the state of the art. However, on the analysis of high-speed and compressible flows, and related to complex geometries, having a robust pipeline for the mesh generation, preprocessing and solving steps is an asset. Moreover, the extraction of the Jacobian matrix of the calculated flow solution is not trivial in those cases, leaving the choices of using a finite-difference approach (with the associated numerical cost and truncation error) or a continuous framework (with the difficulties on the incorporation of turbulence and compressibility models) as the only affordable ones. Using a Finite-Volume flow solver, that is widely validated on the calculation of aerospace configurations and incorporates a large variety of turbulence models, is therefore justified. In addition, the availability of an analytic version of the Jacobian matrix (including turbulence models) eliminates the barriers mentioned above, as a discrete framework can be employed to evaluate the stability of the dynamic system.

The calculation of steady/time averaged solutions was coupled with the solving of the associated eigenvalue problem of the discrete Navier-Stokes linear operator. A complete description and validation of the methodolody can be found in Chapters 2 to 4 . The use of a explicit storage for the large Jacobian matrices of the flow solutions was justified by the advantages on extracting the adjoint eigenvalues and eigenvectors, and the cross-platform possibilities that permitted the reproducibility of the stability analysis results using different eigenvalue problem solvers. The size 
of the eigenvalue problem, hence the total memory requirements, were reduced by subspace projection into a smaller computational domain. This reduction could be evaluated for convergence, but it has been proved that the minimum size is the one that totally contains the core of the instability, namely the structural sensitivity region. An in-house stability solver was developed for a better control of the core of the Krylov iterative process on the solving of the eigenvalue problem, together with a tailored input-output system, as described in Section 3.2. This particular choice was made to have a better insight of the iterative process itself, but the methodology could be easily reproduced with black-box software packages, nowadays available and of common use.

The three validation cases presented in this thesis were chosen to cover the different scenarios normally encountered on stability analysis of complex flows: the sudden geometry expansion of a channel flow and the cylinder wake first instability, examples of a Pitchfork and Hopf bifurcations, where the first is defined by the stationary symmetry breaking of the flow configuration, and the last is characterised by the apparition of unsteady flow oscillations with a limit cycle; and the analysis of steady (base) or time-averaged (mean) flows through the study of the turbulent wake behind a D-shaped cylinder. A stability analysis of the linearised mean flow is a resourceful tool on the analysis of non-steady flows, but restricted to those cases where the limit cycle of the associated instability is close to the frequency of the mean flow.

One of the main contributions of this thesis is the analysis of turbine blade configurations on which base bleed is present, that is covered in Chapter 5. The cooling of the trailing edge of the blade can be done by the ejection of cooler flow through an internal passage, that keeps the thin parts of the blade under the melting point. The ejection of cooling flow at the base region has a great impact in the downstream flow topology, that strongly depends on the intensity of the base bleed and its interaction with the present flow structures. In this work a global mode responsible of a stationary change of direction of the base bleed jet was identified and characterized for a wide range of blowing intensities. The flow configuration was supersonic, hence compressibility effects drived the downstream changes generated by the change in direction of the base bleed jet. This symmetry breaking appears at low jet intensities, with a quick change in the flow topology as the mass flow of the base bleed increases, to finally disappear when the strength of the jet enforces the flow symmetry again. The results were correlated with RANS solutions, that where previously validated with available data on the literature. The unstable eigenvalue was tracked to detect the bifurcation at low and high jet intensities. Despite the two-dimensionality of the problem, the presence of strong compression and expansion waves and a large separated region require a very fine spatial discretisation, that, together with the use of a two-equation turbulence model, generate a large linear operator. In this scenario, the use of domain reduction techniques on the stability analysis was an asset. The initial computational domain was big enough to contain the developing upstream boundary layers and the downstream complex flow 
topology, but the actual size of the domain required to obtain the leading global mode of the instability was much smaller. A proper evaluation of the domain size permitted the solving of the eigenvalue problem in a modest workstation, avoiding the memory issues encountered when analysing the whole computational domain. The results of the analysis showed that the instability was related with the sudden expansion of the cooling slot geometry, reproducing the effects of a confined channel expansion. The characteristics of the flow, namely the strong shear layers and the topology of the recirculation area at the base region, gave the instability a unique and characteristic behaviour, related with its destabilization and re-stabilization. A sensitivity analysis of the global mode provided information that was in line with the bifurcation analyses, also confirming that the core of the instability was located inside the base region. The strong trailing edge shear layers and the cooling jet mixing layers appeared as the most sensitive areas, with the sensitivity gradients to base flow modifications having its greatest values in the base region area. However, the analysis of the sensitivity of the eigenvalue to the application of a steady force suggested that local changes inside the injection chamber may have an effect downstream, and hence possible flow control techniques can arise from local actuation and cooling jet flow modulation.

The analysis was later expanded to different sonic regimes, to cover subsonic, transonic and higher supersonic configurations. To do so, an experimental configuration to be tested in a state-of-the-art wind tunnel was designed, with the aim of validating the numerical data and identifying the global instability with experimental data. A turbine blade was modelled as a no-camber slender body with an ejection slot at the trailing edge, with a leading edge designed to avoid interferences at the three sonic regimes. The model and the wind tunnel walls were reproduced in a numerical simulation, and evaluated for a set of different base bleed intensities. Furthermore, to analyse the influence of the geometry on the apparition of the instability, two different trailing edge shapes were considered. The results of the study served to identify the presence of the instability in the six analysed configurations, which was more prone to appear in trailing edges with rounded edges. These differences between the results for the two geometries were explained by the different formation of a Coanda effect, depending on the shape of the trailing edge. For blunt configurations, the change in direction of the flow was generated by the difference in pressure between upper and lower sides of the turbine blade, but without any further attachment of the flow to a downstream wall. This was called a "mild" Coanda effect. On the contrary, a rounded exit of the cooling slot propitiated the re-attachment of the jet flow downstream, generating the characteristic flow distribution of the Coanda. In addition, "full" Coanda deflections were related with a smoother behaviour of the base region flow, with less abrupt changes and more predictable flow topologies. The sensitivity analysis of these configuration served to identify regions where flow control could be more effective on the control of the instability, exciting or damping it, or even controlling its direction. For the rounded case, the position of the areas of maximum receptivity to steady forcing 
was placed near the wall, suggesting that the placement of local flow control devices (suction/blowing or synthetic jets) could exploit the changes in direction of the flow. However, the thickness of the turbine blades put these options far from a possible experimental validation, and even further from its industrial application. But this information can regardless be very valuable on the prediction and prevention of these effects.

The last part of the thesis was dedicated to the development of eigenvalue sensitivity algorithms to predict the effects of changes in the shape of the body of analysis on the global mode of interest. A theoretical revision of the eigenvalue sensitivity was carried out, which results are also evaluated in Chapter 6. A further development was taken to evaluate the influence of a change in a flow parameter in the eigenvalue of interest. These methodology was later applied to changes in the surface mesh, considering the position of each surface node as an independent parameter of the flow system. An innovative approach was proposed to reduce the calculation of the sensitivity to one single evaluation, despite the number of nodes considered on the analysis, only requiring the calculation of an additional Jacobian matrix and the previous evaluation of the eigenvalue sensitivity to steady forcing. The results of the analysis provide individual information for each surface mesh node, highlighting the effect that a movement of a particular node would have on the eigenvalue. These gradients were evaluated for the cylinder first instability, where the predicted direction for the deformations agreed with what would be physically expected, but also with results of similar studies where the cylinder shape was optimized using different techniques. To prove so, a relatively simple gradient-based optimization process was carried out to evaluate the application of the methodology on an optimization loop, with excellent results. It was possible not only to anticipate or delay the apparition of the first instability of the cylinder wake, but also to control the associated frequency of the vortex shedding. The results, however, highlighted the considerations to be taken in further optimizations, as just simply following the directions of the obtained gradients could generate non-feasible geometries, or reduce the quality of the spatial discretisation up to a point where it was not reusable. Additional work is needed on this regard, if the gradients are to be used in more complex scenarios.

\section{Future perspectives}

All the work completed in this Thesis was constrained by the two-dimensionality of the analysis. The first move towards the tackle of more complex scenarios would be the development of a pure BiGlobal approach on the discrete framework, in which the sensitivity analysis and the global analysis of turbulent flows could be implemented.

The analysis on the base bleed trailing edge is currently undergoing its experimental part, where the flow topology is being characterised and compared with the numerical results. This will serve to evaluate the accuracy of the RANS simulations, 
and eventually correlate the apparition of the global instability with the presented results.

The eigenvalue sensitivity to surface shape modifications is applied in this Thesis through a simple optimization algorithm, that works efficiently in the rounded and smooth surface of a cylindrical body. For its application on abrupt geometries (with concave shapes and straight corners), both the gradients distribution and the optimization algorithm would require additional work. The use of a $H_{1}$ norm for the gradients calculation, instead of the used $L_{2}$ norm, would take into account the geometrical distribution of the surface nodes, smoothing and adapting the gradients accordingly. In a parallel way, the steepest-descent algorithm could be replaced by a more complex and refined gradient-based optimization process, including mesh adaptation or generation to overcome possible mesh deformation issues.

In addition, two main contributions on this thesis, the global stability analysis of a base bleed configuration and the calculation of the sensitivity of the eigenvalue to geometrical changes, have not been combined yet. A detailed surface-shape optimization on a thin trailing edge looks, however, not feasible from a product production point of view. But the evaluation of those regions that are most sensitive to geometrical modifications can indeed serve on the final production line. Moreover, the use of PIV techniques will allow to collect valuable data that could be postprocessed later by means of DMD analysis, with the aim of generating low-order methods to predict flow behaviour on base bleed configurations. The information from the stability and sensitivity analyses can extend the capabilities of the models, generating a promising tool on the prediction of secondary flows in turbine design. 



\section{Bibliography}

[1] D. E. Abbott and S. J. Kline. Experimental Investigation of Subsonic Turbulent Flow Over Single and Double Backward Facing Steps. Journal of Basic Engineering, 84(3):317-325, 1962. Cited on page(s) 45, 46.

[2] F. Abraham, M. Behr, and M. Heinkenschloss. Shape Optimization in Stationary Blood Flow: A Numerical Study of Non-Newtonian Effects. Computer Methods in Biomechanics and Biomedical Engineering, 8:127-137, 2005. Cited on page(s) 94 .

[3] F. Alizard, J.-C. Robinet, and G. Filliard. Sensitivity analysis of optimal transient growth for turbulent boundary layers. European Journal of Mechanics B/Fluids, 49:373-386, 2015. Cited on page(s) 6.

[4] N. Alleborn, K. Nandakumar, H. Raszillier, and F. Durst. Further contributions on the two-dimensional flow in a sudden expansion. Journal of Fluid Mechanics, 330:169-188, 1997. Cited on page(s) 45, 46, 47, 75.

[5] P. R. Amestoy, I. S. Duff, J.-Y. L'Excellent, and J. Koster. A Fully Asynchronous Multifrontal Solver Using Distributed Dynamic Scheduling. SIAM Journal on Matrix Analysis and Applications, 23(1):15-41, jan 2001. Cited on page(s) 35, 79 .

[6] G. Arfken. The Method of Steepest Descents. In Mathematical Methods for Physicists, chapter 7.4, pages 428-436. Academic Press, Orlando, FL, 1985. Cited on page(s) 96, 104.

[7] S. Bagheri, E. Åkervik, L. Brandt, and D. S. Henningson. Matrix-Free Methods for the Stability and Control of Boundary Layers. AIAA Journal, 47(5):1057-1068, 2009. Cited on page(s) 6.

[8] S. Balay, S. Abhyankar, M. Adams, J. Brown, P. Brune, K. Buschelman, L. Dalcin, A. Dener, V. Eijkhout, W. Gropp, D. Kaushik, M. Knepley, D. May, L. C. McInnes, R. T. Mills, T. Munson, K. Rupp, P. Sanan, B. Smith, 
S. Zampini, H. Zhang, and H. Zhang. Petsc users manual. Technical Report ANL-95/11 - Revision 3.10, Argonne National Laboratory, 2018. Cited on page(s) 35.

[9] S. Balay, S. Abhyankar, M. Adams, J. Brown, P. Brune, K. Buschelman, L. Dalcin, V. Eijkhout, W. Gropp, D. Kaushik, M. Knepley, D. May, L. C. McInnes, K. Rupp, B. Smith, S. Zampini, H. Zhang, and H. Zhang. Petsc web page, 2017. Cited on page(s) 35.

[10] S. Balay, W. D. Gropp, L. C. McInnes, and B. F. Smith. Efficient Management of Parallelism in Object Oriented Numerical Software Libraries. In E. Arge, A. M. Bruaset, and H. P. Langtangen, editors, Modern Software Tools in Scientific Computing, pages 163-202. Birkhäuser Press, 1997. Cited on page(s) 35,79 .

[11] D. Barkley. Linear analysis of the cylinder wake mean flow. Europhysics Letters (EPL), 75(5):750-756, 2006. Cited on page(s) 19, 20, 49, 60.

[12] D. Barkley and R. Henderson. Three-dimensional Floquet stability analysis of the wake of a circular cylinder. Journal of Fluid Mechanics, 322:215-241, 1996. Cited on page(s) 5, 102.

[13] F. Battaglia and G. Papadopoulos. Bifurcation Characteristics of Flows in Rectangular Sudden Expansion Channels. Journal of Fluids Engineering, 128(4):671, 2006. Cited on page(s) 45, 75.

[14] F. Battaglia, S. J. Tavener, A. K. Kulkarni, and C. L. Merkle. Bifurcation of Low Reynolds Number Flows in Symmetric Channels. AIAA Journal, 35(1):99-105, jan 1997. Cited on page(s) 47.

[15] D. E. Bohn, V. J. Becker, K. D. Behnke, and B. F. Bonhoff. Experimental and Numerical Investigations of the Aerodynamical Effects of Coolant Injection Through the Trailing Edge of a Guide Vane. In ASME. Turbo Expo: Power for Land, Sea, and Air, Volume 4: Heat Transfer; Electric Power; Industrial and Cogeneration, Houston, Texas, 1995. Cited on page(s) 59.

[16] A. Bottaro, P. Corbett, and P. Luchini. The effect of base flow variation on flow stability. Journal of Fluid Mechanics, 476:293-302, feb 2003. Cited on page(s) 6 .

[17] E. Boujo, A. Fani, and F. Gallaire. Second-order sensitivity in the cylinder wake: Optimal spanwise-periodic wall actuation and wall deformation. Physical Review Fluids, 4(5):053901, may 2019. Cited on page(s) 6.

[18] E. Boujo and F. Gallaire. Controlled reattachment in separated flows: a variational approach to recirculation length reduction. Journal of Fluid Mechanics, 742(2014):618-635, 2014. Cited on page(s) 6. 
[19] J. Boussinesq. Essai sur la theorie des eaux courantes. In Memoires presentes par divers savants a l'Academie des Sciences de l'Institut National de France, volume Tome XXIII. Imprimerie Nationale, Paris, 1877. Cited on page(s) 29.

[20] O. M. F. Browne, G. Rubio, E. Ferrer, and E. Valero. Sensitivity analysis to unsteady perturbations of complex flows: a discrete approach. International Journal for Numerical Methods in Fluids, 76(12):1088-1110, dec 2014. Cited on page(s) 21, 33, 35, 42, 44, 95.

[21] D. Canuto and K. Taira. Two-dimensional compressible viscous flow around a circular cylinder. Journal of Fluid Mechanics, 785(1956):349-371, dec 2015. Cited on page(s) 102.

[22] W. Cherdron, F. Durst, and J. H. Whitelaw. Asymmetric flows and instabilities in symmetric ducts with sudden expansions. Journal of Fluid Mechanics, 84(1):13-31, jan 1978. Cited on page(s) 45.

[23] J.-M. Chomaz. Global Instabilities in Spatially Developing Flows: NonNormality and Nonlinearity. Annual Review of Fluid Mechanics, 37(1):357392, 2005. Cited on page(s) 6, 17.

[24] V. Citro, L. Siconolfi, D. Fabre, F. Giannetti, and P. Luchini. Stability and Sensitivity Analysis of the Secondary Instability in the Sphere Wake. AIAA Journal, (2):1-8, 2017. Cited on page(s) 6.

[25] J. D. Crouch, A. Garbaruk, and D. Magidov. Predicting the onset of flow unsteadiness based on global instability. Journal of Computational Physics, 224(2):924-940, 2007. Cited on page(s) 43.

[26] J. D. Crouch, A. Garbaruk, D. Magidov, and A. Travin. Origin of transonic buffet on aerofoils. Journal of Fluid Mechanics, 628:357, 2009. Cited on page(s) 6 .

[27] J. D. Crouch, A. Garbaruk, and M. Strelets. Global Instability Analysis of Unswept- and Swept-Wing Transonic Buffet Onset. In 2018 Fluid Dynamics Conference, number June, Reston, Virginia, jun 2018. American Institute of Aeronautics and Astronautics. Cited on page(s) 6.

[28] J. de Vicente, J. Basley, F. Meseguer-Garrido, J. Soria, and V. Theofilis. Three-dimensional instabilities over a rectangular open cavity: from linear stability analysis to experimentation. Journal of Fluid Mechanics, 748(2014):189220, 2014. Cited on page(s) 6.

[29] J. De Vicente, D. Rodríguez, V. Theofilis, and E. Valero. Stability analysis in spanwise-periodic double-sided lid-driven cavity flows with complex cross-sectional profiles. Computers and Fluids, 43(1):143-153, 2011. Cited on page(s) 35 . 
[30] S. R. N. De Zilwa, L. Khezzar, and J. H. Whitelaw. Flows through plane sudden-expansions. International Journal for Numerical Methods in Fluids, 32(3):313-329, feb 2000. Cited on page(s) 46.

[31] B. H. Dennis, G. S. Dulikravich, and Z.-X. Han. Constrained Shape Optimization of Airfoil Cascades Using a Navier-Stokes Solver and a Genetic/SQP Algorithm. In ASME. Turbo Expo: Power for Land, Sea, and Air, Volume 1: Aircraft Engine; Marine; Turbomachinery; Microturbines and Small Turbomachinery, Indianapolis, jun 1999. ASME. Cited on page(s) 94.

[32] J. D. Denton and L. Xu. The Trailing Edge Loss of Transonic Turbine Blades. Journal of Turbomachinery, 112(2):277-285, 1990. Cited on page(s) 58, 59.

[33] D. Drikakis. Bifurcation phenomena in incompressible sudden expansion flows. Physics of Fluids, 9(1):76-87, 1997. Cited on page(s) 46.

[34] F. Durst, W. F. Schierholz, and A. M. Wunderlich. Experimental and Numerical Investigations of Plane Duct Flows With Sudden Contraction. Journal of Fluids Engineering, 109(4):376-373, 1987. Cited on page(s) 8.

[35] R. P. Dwight. Efficiency Improvements of RANS-Based Analysis and Optimization Using Implicit and Adjoint Methods on Unstrucutured Grids. Phd thesis, University of Manchester, 2006. Cited on page(s) xiii, 32.

[36] D. Fabre, D. Ferreira-Sabino, V. Citro, J. Sierra, M. Pigou, F. Giannetti, and P. Bonnefis. A practical review on linear and nonlinear global approaches to flow instabilities. Applied Mechanics Reviews, 70(November):1-16, 2018. Cited on page(s) 6 .

[37] A. Fani, S. Camarri, and M. V. Salvetti. Stability analysis and control of the flow in a symmetric channel with a sudden expansion. Physics of Fluids, 24(8):084102, aug 2012. Cited on page(s) xiv, 6, 46, 47, 48, 62, 70, 73, 87.

[38] R. M. Fearn, T. Mullin, and K. A. Cliffe. Nonlinear flow phenomena in a symmetric sudden expansion. Journal of Fluid Mechanics, 211(-1):595, feb 1990. Cited on page(s) 46, 75 .

[39] E. Ferrer, O. M. Browne, and E. Valero. Sensitivity analysis to control the far-wake unsteadiness behind turbines. Energies, 10(10):1-21, 2017. Cited on page(s) 6, 95 .

[40] E. Ferrer, J. de Vicente, and E. Valero. Low cost 3D global instability analysis and flow sensitivity based on dynamic mode decomposition and highorder numerical tools. International Journal for Numerical Methods in Fluids, 76(3):169-184, sep 2014. Cited on page(s) 6, 35. 
[41] M. Fosas de Pando, D. Sipp, and P. J. Schmid. Efficient evaluation of the direct and adjoint linearized dynamics from compressible flow solvers. Journal of Computational Physics, 231(23):7739-7755, 2012. Cited on page(s) 36.

[42] F. Fraysse, E. Valero, and G. Rubio. Quasi-a priori truncation error estimation and higher order extrapolation for non-linear partial differential equations. Journal of Computational Physics, 253(November):389-404, 2013. Cited on page(s) 63 .

[43] F. Gallaire and P.-T. Brun. Fluid dynamic instabilities: theory and application to pattern forming in complex media. Philosophical Transactions of the Royal Society A: Mathematical, Physical and Engineering Sciences, 375(2093):20160155, 2017. Cited on page(s) 3.

[44] J. H. Gerrard. The mechanics of the formation of vortices behind bluff bodies. Journal of Fluid Mechanics, 25:401-413, 1966. Cited on page(s) 5.

[45] F. Giannetti, S. Camarri, and V. Citro. Sensitivity analysis and passive control of the secondary instability in the wake of a cylinder. Journal of Fluid Mechanics, 864:45-72, 2019. Cited on page(s) 5, 6 .

[46] F. Giannetti and P. Luchini. Structural sensitivity of the first instability of the cylinder wake. Journal of Fluid Mechanics, 581:167, 2007. Cited on page(s) xiii, 6, 36, 43, 44, 86, 94, 102.

[47] Y. Gorbachova, E. Valero, A. Martinez-Cava, G. Paniagua, and B. H. Saracoglu. Study of the attainable flow topologies in a supersonic blunt trailing edge at various blowing ratios. In AIAA SciTech Forum 2016, 2016. Cited on page(s) 61,65 .

[48] M. Grilli, P. J. Schmid, S. Hickel, and N. A. Adams. Analysis of unsteady behaviour in shockwave turbulent boundary layer interaction. Journal of Fluid Mechanics, 700(700):16-28, jun 2012. Cited on page(s) 60.

[49] Y. Guevel, T. Allain, G. Girault, and J. Cadou. Numerical bifurcation analysis for 3-dimensional sudden expansion fluid dynamic problem. International Journal for Numerical Methods in Fluids, 87(1):1-26, may 2018. Cited on page(s) 46.

[50] F. Guiho, F. Alizard, and J.-C. Robinet. Instabilities in oblique shock wave/laminar boundary-layer interactions. Journal of Fluid Mechanics, 789:135, 2017. Cited on page(s) 6 .

[51] F. R. Hama. Experimental Studies on the Lip Shock. AIAA Journal, 6(2):212219, 1968. Cited on page(s) 59. 
[52] T. Hawa and Z. Rusak. The dynamics of a laminar flow in a symmetric channel with a sudden expansion. Journal of Fluid Mechanics, 436:283-320, 2001. Cited on page(s) 45.

[53] W. He, A. Burtsev, V. Theofilis, K. Zhang, K. Taira, S. Hayostek, and M. Amitay. Wake Dynamics of Finite Aspect Ratio Wings. Part III: TriGlobal Linear Stability Analysis. In AIAA Scitech 2019 Forum, Reston, Virginia, jan 2019. American Institute of Aeronautics and Astronautics. Cited on page(s) 5.

[54] R. D. Henderson and D. Barkley. Secondary instability in the wake of a circular cylinder. American Institute of Physics, 8(6):1683-1685, 1996. Cited on page(s) 5 .

[55] J. L. Herrin and J. C. Dutton. Supersonic base flow experiments in the near wake of a cylindrical afterbody. AIAA Journal, 32(1):77-83, 1994. Cited on page(s) 59 .

[56] V. Heuveline and F. Strauß. Shape optimization towards stability in constrained hydrodynamic systems. Journal of Computational Physics, 228(4):938-951, 2009. Cited on page(s) 95.

[57] R. A. Horn and C. R. Johnson. Matrix Analysis. Cambridge University Press, second edi edition, 2013. Cited on page(s) 18, 34.

[58] P. Huerre and M. Rossi. Hydrodynamic instabilities in open flows. In C. Godreche and P. Manneville, editors, Hydrodynamics and Nonlinear Instabilities, pages 81-294. Cambridge University Press, Cambridge. Cited on page(s) 8.

[59] H. E. Huppert. Flow and instability of a viscous current down a slope. Nature, 300(5891):427-429, dec 1982. Cited on page(s) 3.

[60] A. K. Hussain and W. C. Reynolds. The mechanics of an organized wave in turbulent shear flow. Journal of Fluid Mechanics, 41(2):241-258, 1970. Cited on page(s) 19.

[61] M. C. Iorio, L. M. González, and E. Ferrer. Direct and adjoint global stability analysis of turbulent transonic flows over a NACA0012 profile. International Journal for Numerical Methods in Fluids, 76(3):147-168, 2014. Cited on page(s) $6,35,60$.

[62] M. C. Iorio, L. M. González, and A. Martínez-Cava. Global Stability Analysis of a Compressible Turbulent Flow Around a High-Lift Configuration. AIAA Journal, 54(2):373-385, feb 2016. Cited on page(s) 60.

[63] L. Jacquin, P. Molton, S. Deck, B. Maury, and D. Soulevant. Experimental Study of Shock Oscillation over a Transonic Supercritical Profile. AIAA Journal, 47(9):1985-1994, sep 2009. Cited on page(s) 6. 
[64] L. E. Jones, R. D. Sandberg, and N. D. Sandham. Stability and receptivity characteristics of a laminar separation bubble on an aerofoil. Journal of Fluid Mechanics, 648(2):257, apr 2010. Cited on page(s) 5.

[65] M. P. Juniper, A. Hanifi, and V. Theofilis. Modal Stability Theory. Applied Mechanics Reviews, 66(2):021004, 2014. Cited on page(s) 6, 8.

[66] M. P. Juniper, O. Tammisola, and F. Lundell. The local and global stability of confined planar wakes at intermediate Reynolds number. Journal of Fluid Mechanics, 686:218-238, nov 2011. Cited on page(s) 20.

[67] V. Kitsios, D. Rodríguez, V. Theofilis, A. Ooi, and J. Soria. BiGlobal stability analysis in curvilinear coordinates of massively separated lifting bodies. Journal of Computational Physics, 228:7181-7196, 2009. Cited on page(s) 5.

[68] F. H. Kost and A. T. Holmes. Aerodynamic effect of coolant ejection in the rear part of transonic rotor blades. In AGARD Heat transfer and Cooling in Gas Turbines 12 p (SEE N86-29823 21-07), 1985. Cited on page(s) 59.

[69] I. Lashgari, O. Tammisola, V. Citro, M. P. Juniper, and L. Brandt. The planar X-junction flow: stability analysis and control. Journal of Fluid Mechanics, 753(August):1-28, 2014. Cited on page(s) 60, 62, 95.

[70] B. H. K. Lee. Self-sustained shock oscillations on airfoils at transonic speeds. Progress in Aerospace Sciences, 37(2):147-196, 2001. Cited on page(s) 6.

[71] R. B. Lehoucq and A. G. Salinger. Massively parallel linear stability analysis with P_ARPACK for 3D fluid flow modeled with MPSalsa. pages 286-295. 1998. Cited on page(s) 79.

[72] R. B. Lehoucq, D. C. Sorensen, and C. Yang. ARPACK Users' Guide. Society for Industrial and Applied Mathematics, Philadelphia, PA, 1998. Cited on page(s) 35.

[73] L. Leifsson and S. Koziel. Aerodynamic shape optimization by variable-fidelity computational fluid dynamics models: A review of recent progress. Journal of Computational Science, 10:45-54, sep 2015. Cited on page(s) 94.

[74] F. Lozano, J. Saavedra, G. Paniagua, A. Martinez-Cava, and E. Valero. Experimental Approach to Characterize Base Region Coanda Flow Topology Induced by Pulsating Blowing. In 8th European Conference for Aeronautics and Space Sciences (EUCASS), Madrid, 2019. Cited on page(s) 2.

[75] P. Luchini and A. Bottaro. Adjoint Equations in Stability Analysis. Annual Review of Fluid Mechanics, 46(1):493-517, 2014. Cited on page(s) 6, 95. 
[76] P. Manneville. Rayleigh-Bénard Convection: Thirty Years of Experimental, Theoretical, and Modeling Work. Number June, pages 41-65. 2006. Cited on page(s) 3 .

[77] O. Marquet, D. Sipp, and L. Jacquin. Sensitivity analysis and passive control of cylinder flow. Journal of Fluid Mechanics, 615(2008):221-252, 2008. Cited on page(s) xiii, 6, 43, 44, 45, 46, 87, 95, 102, 103.

[78] A. L. Marsden, M. Wang, J. E. Dennis, and P. Moin. Suppression of vortexshedding noise via derivative-free shape optimization. Physics of Fluids, 16(10), 2004. Cited on page(s) 94.

[79] A. Martinez-Cava, E. Valero, J. de Vicente, and G. Paniagua. Coanda Flow Characterization on Base Bleed Configurations Using Global Stability Analysis. In AIAA Scitech 2019 Forum, San Diego, California, jan 2019. American Institute of Aeronautics and Astronautics. Cited on page(s) 1, 58, 77, 82, 84 .

[80] A. Martinez-Cava, E. Valero, J. Saavedra, F. Lozano, and G. Paniagua. Optimization of the base bleed to control trailing edge flows in a wide range of Mach numbers. In 8th European Conference for Aeronautics and Space Sciences (EUCASS), Madrid, 2019. Cited on page(s) 1.

[81] A. Martinez-Cava, E. Valero, and J. D. Vicente. Sensitivity Analysis of Supersonic Turbine Trailing Edges. In Proceedings of 7th European Conference on Computational Fluid Dynamics (ECFD 7), pages 502-518. International Center for Numerical Methods in Engineerin (CIMNE), Glasgow, UK, 2018. Cited on page(s) 1, 58, 77.

[82] A. Martinez-Cava, Y. Wang, J. de Vicente, and E. Valero. Pressure Bifurcation Phenomenon on Supersonic Blowing Trailing Edges. AIAA Journal, 57(1):153-164, jan 2019. Cited on page(s) 1, 58.

[83] S. Melber and R. Heinrich. Low Mach-Number Preconditioning for the DLRTAU Code and Application to High-Lift Flows. In C. Breitsamter, B. Laschka, H. Heinemann, and R. Hilbig, editors, New Results in Numerical and Experimental Fluid Mechanics IV. Notes on Numerical Fluid Mechanics and Multidisciplinary Design, vol 87, pages 390-397. Springer, Berlin, Heidelberg, 2004. Cited on page(s) 46.

[84] P. Meliga and J.-M. Chomaz. Global modes in a confined impinging jet: Application to heat transfer and control. Theoretical and Computational Fluid Dynamics, 25(1-4):179-193, 2011. Cited on page(s) 6.

[85] P. Meliga, J.-M. Chomaz, and D. Sipp. Unsteadiness in the wake of disks and spheres: Instability, receptivity and control using direct and adjoint global stability analyses. Journal of Fluids and Structures, 25(4):601-616, 2009. Cited on page(s) 95. 
[86] P. Meliga, G. Pujals, and É. Serre. Sensitivity of 2-D turbulent flow past a D-shaped cylinder using global stability. Physics of Fluids, 24(6), 2012. Cited on page(s) xiv, 6, 49, 52, 53 .

[87] P. Meliga, D. Sipp, and J.-M. Chomaz. Open-loop control of compressible afterbody flows using adjoint methods. Physics of Fluids, 22(5):1-18, may 2010. Cited on page(s) 6, 95.

[88] C. Mettot. Stabilité linéaire, sensibilité et contrôle passif d'écoulements turbulents par différence finies. PhD thesis, 2013. Cited on page(s) xiv, 44, 45, $49,51,52$.

[89] C. Mettot, F. Renac, and D. Sipp. Computation of eigenvalue sensitivity to base flow modifications in a discrete framework: Application to open-loop control. Journal of Computational Physics, 269:234-258, 2014. Cited on page(s) $6,21,42,95$.

[90] C. Mettot, D. Sipp, and H. Bézard. Quasi-laminar stability and sensitivity analyses for turbulent flows: Prediction of low-frequency unsteadiness and passive control. Physics of Fluids, 26(4), 2014. Cited on page(s) xiv, 49, 50, 52,53 .

[91] J. Mizushima and Y. Shiotani. Transitions and instabilities of flow in a symmetric channel with a suddenly expanded and contracted part. Journal of Fluid Mechanics, 434(May):355-369, may 2001. Cited on page(s) 70.

[92] F. Motallebi and J. Norbury. The effect of base bleed on vortex shedding and base pressure in compressible flow. Journal of Fluid Mechanics, 110:273-292, 1981. Cited on page(s) $75,80$.

[93] J. F. Nash. A Review of Research on Two-Dimensional Base Flow. ARC $R \mathscr{E} M,(3323): 1-25,1963$. Cited on page(s) 59.

[94] J. F. Nash. A Discussion of Two-Dimensional Turbulent Base Flows. Technical Report 3468, Aeronautical Research Council, Great Britain, 1967. Cited on page(s) 59 .

[95] J. F. Nash, V. G. Quincey, and J. Callinan. Experiments on Two-Dimensional Base Flow at Subsonic and Transonic Speeds. Technical Report 3427, Aeronautical Research Council, Great Britain, 1966. Cited on page(s) 59.

[96] J. W. Nichols, G. V. Candler, A. Dwivedi, N. Hildebrand, and M. R. Jovanović. Simulation and stability analysis of oblique shock-wave/boundary-layer interactions at Mach 5.92. Physical Review Fluids, 3(1):1-23, 2018. Cited on page(s) 6. 
[97] B. R. Noack and H. Eckelmann. A global stability analysis of the steady and periodic cylinder wake. Journal of Fluid Mechanics, 270(-1):297, jul 1994. Cited on page(s) 5, 42, 102.

[98] K. Oberleithner, L. Rukes, and J. Soria. Mean flow stability analysis of oscillating jet experiments. Journal of Fluid Mechanics, 757:1-32, 2014. Cited on page(s) 6, 19, 20, 60 .

[99] G. Paniagua, D. Cuadrado, J. Saavedra, V. Andreoli, D. Lawrence, T. Meyer, and S. Meyer. Design of the Purdue Experimental Turbine Aerothermal Laboratory for Optical and Surface Aero-Thermal Measurements. In ASME. Turbo Expo: Power for Land, Sea, and Air, Volume 6: Ceramics; Controls, Diagnostics and Instrumentation; Education; Manufacturing Materials and Metallurgy, 2016. Cited on page(s) 76.

[100] G. Paniagua, T. Yasa, A. D. La Loma, L. Castillon, and T. Coton. Unsteady Strong Shock Interactions in a Transonic Turbine: Experimental and Numerical Analysis. Journal of Propulsion and Power, 24(4):722-731, jul 2008. Cited on page(s) 58.

[101] V. Parezanović and O. Cadot. Experimental sensitivity analysis of the global properties of a two-dimensional turbulent wake. Journal of Fluid Mechanics, 693:115-149, feb 2012. Cited on page(s) xiv, 49, 50, 52, 53.

[102] M. Pastor, M. Binda, and T. Hararik. Modal Assurance Criterion. Procedia Engineering, 48:543-548, 2012. Cited on page(s) 105.

[103] E. W. Perkins, L. H. Jorgensen, and S. C. Sommer. NACA-TR-1386: Investigation of the drag of various axially symmetric nose shapes of fineness ratio 3 for Mach numbers from 1.24 to 7.4. Technical report, National Advisory Committee for Aeronautics. Ames Aeronautical Lab.; Moffett Field, CA, United States, 1958. Cited on page(s) 76.

[104] J. E. Peter and R. P. Dwight. Numerical sensitivity analysis for aerodynamic optimization: A survey of approaches. Computers and Fluids, 39(3):373-391, 2010. Cited on page(s) 94.

[105] B. Pier. On the frequency selection of finite-amplitude vortex shedding in the cylinder wake. Journal of Fluid Mechanics, 458:407-417, 2002. Cited on page(s) 20 .

[106] M. Provansal, C. Mathis, and L. Boyer. Bénard-von Kármán instability: transient and forced regimes. Journal of Fluid Mechanics, 182:1-22, sep 1987. Cited on page(s) 42 . 
[107] M. Raffel and F. Kost. Investigation of aerodynamic effects of coolant ejection at the trailing edge of a turbine blade model by PIV and pressure measurements. Experiments in Fluids, 24(5-6):447-461, 1998. Cited on page(s) 59, 75 .

[108] S. C. Reddy and D. S. Henningson. Energy Growth in Viscous Channel Flows. Journal of Fluid Mechanics, 252:209-238, 1993. Cited on page(s) 5.

[109] O. Reynolds. An experimental investigation of the circumstances which determine whether the motion of water shall be direct or sinuous, and of the law of resistance in parallel channels. Philosophical Transactions of the Royal Society of London, 174:935-982, 1883. Cited on page(s) 4.

[110] J. Robinet. Bifurcations in Shock Wave / Laminar Boundary Layer Interaction: Global Instability Approach. Journal of Fluid Mechanics, 2006. Cited on page(s) 6 .

[111] C. W. Rowley, T. Colonius, and A. J. Basu. On self-sustained oscillations in two-dimensional compressible flow over rectangular cavities. Journal of Fluid Mechanics, 455:315-346, 2002. Cited on page(s) 5.

[112] Y. Saad. Analysis of Some Krylov Subspace Approximations. SIAM Journal of Numerical Analysis, 29(1):209-228, 1992. Cited on page(s) 33.

[113] Y. Saad. Iterative Methods for Sparse Linear Systems, volume 3. 2003. Cited on page(s) 33.

[114] Y. Saad. Numerical Methods for Large Eigenvalue Problems. Society for Industrial and Applied Mathematics, 2011. Cited on page(s) 33, 34.

[115] Y. Saad and M. H. Schultz. GMRES: A Generalized Minimal Residual Algorithm for Solving Nonsymmetric Linear Systems. SIAM Journal on Scientific and Statistical Computing, 7(3):856-869, jul 1986. Cited on page(s) 34.

[116] Y. Saad and H. A. van der Vorst. Iterative solution of linear systems in the 20th century. Journal of Computational and Applied Mathematics, 123(1):133, 2000. Cited on page(s) 33.

[117] R. D. Sandberg. Numerical investigation of turbulent supersonic axisymmetric wakes. Journal of Fluid Mechanics, 702(July):488-520, 2012. Cited on page(s) 6,59 .

[118] R. D. Sandberg and H. F. Fasel. Numerical investigation of transitional supersonic axisymmetric wakes. Journal of Fluid Mechanics, 563:1-41, 2006. Cited on page(s) 6, 59 . 
[119] S. Sanvido. Stability Analysis of Complex Aerodynamic Flows. PhD thesis, UPM, 2017. Cited on page(s) 36, 64 .

[120] S. Sanvido, J. Garicano-Mena, J. de Vicente, and E. Valero. Critical Assessment of Domain Reduction Strategies for Global Stability Analisis. Under review by Communications in Computational Physics, 2019. Cited on page(s) 35,86 .

[121] B. H. Saracoglu, G. Paniagua, S. Salvadori, F. Tomasoni, S. Duni, T. Yasa, and A. Miranda. Trailing edge shock modulation by pulsating coolant ejection. Applied Thermal Engineering, 48(April):1-10, 2012. Cited on page(s) 59, 74.

[122] B. H. Saracoglu, G. Paniagua, J. Sanchez, and P. Rambaud. Effects of blunt trailing edge flow discharge in supersonic regime. Computers $\& 5$ Fluids, 88(September):200-209, dec 2013. Cited on page(s) xiv, 58, 59, 60, 61, $65,67,73,75,77,84$.

[123] F. Sartor, C. Mettot, R. Bur, and D. Sipp. Unsteadiness in transonic shockwave/boundary-layer interactions: experimental investigation and global stability analysis. Journal of Fluid Mechanics, 781(October):550-577, 2015. Cited on page(s) 6, 60 .

[124] F. Sartor, C. Mettot, R. Bur, and D. Sipp. Unsteadiness in transonic shockwave/boundary-layer interactions: experimental investigation and global stability analysis. Journal of Fluid Mechanics, 781:550-577, 2017. Cited on page(s) 6 .

[125] P. J. Schmid. Nonmodal Stability Theory. Annual Review of Fluid Mechanics, 39:129-62, 2007. Cited on page(s) 6.

[126] P. J. Schmid and L. Brandt. Analysis of fluid systems: stability, receptivity, sensitivity. Applied Mechanics Reviews, 66(2), 2014. Cited on page(s) 6, 94.

[127] P. J. Schmid, M. F. de Pando, and N. Peake. Stability analysis for n-periodic arrays of fluid systems. Physical Review Fluids, 2(11):113902, nov 2017. Cited on page(s) 1.

[128] F. G. Schmitt. About Boussinesq's turbulent viscosity hypothesis: historical remarks and a direct evaluation of its validity. Comptes Rendus Mécanique, 335(9-10):617-627, sep 2007. Cited on page(s) 29.

[129] D. Schwamborn, T. Gerhold, and R. Heinrich. The DLR Tau-code: Recent Applications in Research and Industry. In ECCOMAS CFD 2006: Proceedings of the European Conference on Computational Fluid Dynamics. Delft University of Technology, Edmond aan Zee, Netherlands, 2006. Cited on page(s) 27. 
[130] L. Siconolfi, V. Citro, F. Giannetti, S. Camarri, and P. Luchini. Towards a quantitative comparison between global and local stability analysis. Journal of Fluid Mechanics, 819:147-164, 2017. Cited on page(s) 5.

[131] C. H. Sieverding, M. Stanislas, and J. Snoek. The Base Pressure Problem in Transonic Turbine Cascades. Journal of Engineering for Power, 102(3):711718, 1979. Cited on page(s) 59.

[132] D. Sipp and A. Lebedev. Global stability of base and mean flows: a general approach and its applications to cylinder and open cavity flows. Journal of Fluid Mechanics, 593(December):333-358, 2007. Cited on page(s) 20, 60.

[133] D. Sipp, O. Marquet, P. Meliga, and A. Barbagallo. Dynamics and Control of Global Instabilities in Open-Flows: A Linearized Approach. Applied Mechanics Reviews, 63(3):030801, 2010. Cited on page(s) 95.

[134] S. N. Skinner and H. Zare-Behtash. State-of-the-art in aerodynamic shape optimisation methods. Applied Soft Computing Journal, 62:933-962, 2018. Cited on page(s) 94 .

[135] R. Smyth. Turbulent Flow Over a Plane Symmetric Sudden Expansion. Journal of Fluids Engineering, 101(3):348, 1979. Cited on page(s) 46.

[136] P. R. Spalart and S. R. Allmaras. A one-equation turbulence model for aerodynamic flows. In 30th Aerospace Sciences Meeting and Exhibit, Reston, Virigina, jan 1992. American Institute of Aeronautics and Astronautics. Cited on page(s) 29, 49.

[137] K. R. Sreenivasan, P. J. Strykowski, and D. J. Olinger. Hopf bifurcation, Landau equation, and vortex shedding behind circular cylinders. In Forum on Unsteady Flow Separation, pages 1-13. American Society of Mechanical Engineers, 1987. Cited on page(s) 5, 8, 42.

[138] P. J. Strykowski and K. R. Sreenivasan. On the formation and suppression of vortex 'shedding' at low Reynolds numbers. Journal of Fluid Mechanics, 218:71-107, 1990. Cited on page(s) 95.

[139] K. Taira, S. L. Brunton, S. T. M. Dawson, C. W. Rowley, T. Colonius, B. J. McKeon, O. T. Schmidt, S. Gordeyev, V. Theofilis, and L. S. Ukeiley. Modal Analysis of Fluid Flows: An Overview. AIAA Journal, 55(12):4013-4041, 2017. Cited on page(s) 6, 95.

[140] O. Tammisola. Optimal wavy surface to suppress vortex shedding using second-order sensitivity to shape changes. European Journal of Mechanics, B/Fluids, 62:139-148, 2017. Cited on page(s) 6, 95. 
[141] O. Tammisola, F. Giannetti, V. Citro, and M. P. Juniper. Second-order perturbation of global modes and implications for spanwise wavy actuation. Journal of Fluid Mechanics, 755:314-335, 2014. Cited on page(s) 6, 95.

[142] O. Tammisola and M. P. Juniper. Coherent structures in a swirl injector at $\mathrm{Re}=4800$ by nonlinear simulations and linear global modes. Journal of Fluid Mechanics, 792:620-657, apr 2016. Cited on page(s) 6, 20.

[143] M. Tanner. Reduction of base drag. Progress in Aerospace Sciences, 16(4):369384, 1975. Cited on page(s) 75.

[144] V. Theofilis. Global Linear Instability. Annual Review of Fluid Mechanics, 43(1):319-352, 2011. Cited on page(s) 6, 8.

[145] V. Theofilis and T. Colonius. Three-dimensional instabilities of compressible flow over open cavities: Direct solution of the biglobal eivenvalue problem. In 34th AIAA Fluid Dynamics Conference and Exhibit, 2004. Cited on page(s) 6.

[146] C. Thomas, S. Mughal, H. Roland, R. Ashworth, and A. Martinez-Cava. Effect of small surface deformations on the stability of tollmien-schlichting disturbances. AIAA Journal, 56(6), 2018. Cited on page(s) 2.

[147] S. Timme. Global Shock Buffet Instability on NASA Common Research Model. In AIAA Scitech 2019 Forum, Reston, Virginia, jan 2019. American Institute of Aeronautics and Astronautics. Cited on page(s) 6.

[148] . Unidata. Network Common Data Form (NetCDF) [software]. Boulder, CO: UCAR/Unidata Program Center. (https://doi.org/10.5065/D6H70CW6). Cited on page(s) 32 .

[149] E. Valero, E. Ferrer, and J. de Vicente. Numerical Methods for Direct Numerical Simulation and Stability Analysis. In E. Valero and F. Pinna, editors, Progress in flow instability analysis and laminar-turbulent transition modeling, VKI LS 2014-0\%. Von Karman Institute for Fluid Dynamics, 2014. Cited on page(s) 70 .

[150] J. van Ingen. The eN Method for Transition Prediction. Historical Review of Work at TU Delft. In 38th Fluid Dynamics Conference and Exhibit, Reston, Virigina, jun 2008. American Institute of Aeronautics and Astronautics. Cited on page(s) 5.

[151] C. Wales, A. L. Gaitonde, D. P. Jones, D. Avitabile, and A. R. Champneys. Numerical continuation of high Reynolds number external flows. International Journal for Numerical Methods in Fluids, 68(2):135-159, jan 2012. Cited on page(s) 5 . 
[152] Y. Wang, E. Ferrer, and A. Martinez-cava. Enhanced stability of flows through contraction channels: combining shape optimisation and linear stability analysis. Under review by Physics of Fluids, 2019. Cited on page(s) 2.

[153] Y. Wang, E. Valero, Y. Zheng, E. Ferrer, E. Problem, C. Channel, and S. Analysis. Eigenmode Optimization of a Contraction Channel Based on Stability Analysis. In J. R. Roger Owen, Rene de Borst and C. Pearce, editors, Proceedings of 7th European Conference on Computational Fluid Dynamics (ECFD 7), number June, pages 4299-4310. International Center for Numerical Methods in Engineerin (CIMNE), Glasgow, UK, 2018. Cited on page(s) 1, 95.

[154] Y. Wang and L. Zhao. Investigation on the effect of trailing edge ejection on a turbine cascade. Applied Mathematical Modelling, 37(9):6254-6265, 2013. Cited on page(s) 59 .

[155] D. C. Wilcox. Formulation of the k-w Turbulence Model Revisited. AIAA Journal, 46(11):2823-2838, nov 2008. Cited on page(s) 29.

[156] C. H. K. Williamson. Vortex dynamics in the cylinder wake. Annual Review of Fluid Mechanics, (28):477-539, 1996. Cited on page(s) 5, 42.

[157] K. Wu, Y. Saad, and A. Stathopoulos. Preconditioned Krylov Subspace Methods for Eigenvalue Problems. In Copper Mountain Conference on Iterative methods, volume 1, pages 1-6, 1996. Cited on page(s) 33.

[158] G. Zhou, K. Xu, and F. Liu. Grid-converged solution and analysis of the unsteady viscous flow in a two-dimensional shock tube. Physics of Fluids, 30(1):016102, 2018. Cited on page(s) 78.

[159] D. W. Zingg, M. Nemec, and T. H. Pulliam. A comparative evaluation of genetic and gradient-based algorithms applied to aerodynamic optimization. Revue européenne de mécanique numérique, 17(1-2):103-126, 2008. Cited on page(s) 94. 


